\title{
Dynamic Light Scattering on Complex Plasmas
}

\author{
Dissertation \\ zur \\ Erlangung des Grades \\ eines Doktors der Naturwissenschaften \\ in der Fakultät für Physik und Astronomie \\ der Ruhr-Universität Bochum
}

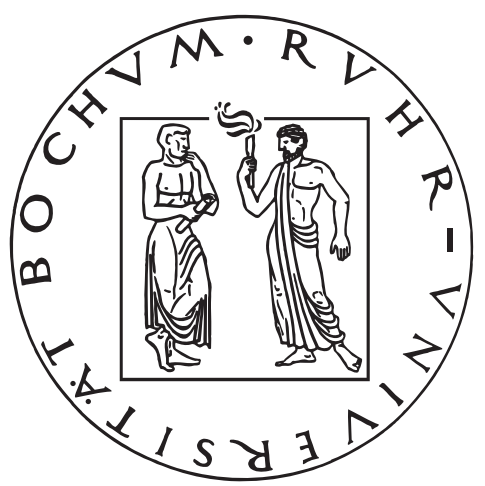

vorgelegt von

Andreas Aschinger

Bochum 2011 
1. Gutachter: Prof. Dr. J. Winter

2. Gutachter: Prof. Dr. H. Soltwisch

Tag der Disputation: 26.01.2012 
To my family. 



\section{Contents}

1 Introduction 1

2 Dynamic Light Scattering 5

2.1 Fluctuations and Time Autocorrelation Functions . . . . . . . . . . . 6

2.1.1 Time Autocorrelation Functions . . . . . . . . . . . . . . 6

2.1.2 Common Forms of Autocorrelation Functions . . . . . . . . . . . . 9

2.2 Basics of Dvnamic Light Scattering . . . . . . . . . . . . . . . . . 10

2.2.1 Principles of Dvnamic Light Scattering . . . . . . . . . . . . . . 10

2.2.2 Dynamic Light Scattering Techniques . . . . . . . . . . . . . . . . . . . . . . . . . . .

2.2 .3 The Scattering Vector $\mathrm{q}$. . . . . . . . . . . . . . . . . . . . 17

2.2 .4 The Coherence Area $\ldots \ldots \ldots \ldots$. . . . . . . . . . . . . . . . . . . . . . . . . . . . . . . . 18

2.2 .5 Number Fluctuations . . . . . . . . . . . . . . . . . . . . . . . . . 19

2.3 Theoretical Forms . . . . . . . . . . . . . . . . . . . . 20

2.3.1 Space Time Correlation Function of the Particle Density . . . . . . . . . 20

2.3.2 Diffusive and Ballistic Motion of Independent Particles . . . . . . . . . . 22

3 Complex Plasmas $\quad 27$

3.1 Charging of Dust Grains in a Plasma . . . . . . . . . . . . . . . 28

3.1 .1 OML Theory . . . . . . . . . . . . . . . . . . . . . 28

3.1 .2 Extensions of the OML Approach . . . . . . . . . . . . . . . 30

3.1 .3 Other Charging Effects . . . . . . . . . . . . . . . . . . . 36

3.1.4 Temporal Behavior of the Dust Grain Charge . . . . . . . . . . . . . . . . 37

3.2 Forces Acting on a Dust Particle . . . . . . . . . . . . . . . . . . . 37

3.2.1 Electrostatic Interaction between Dust Particles . . . . . . . . . . . . . 38

3.2 .2 Ion Drag Force $\ldots \ldots \ldots$. . . . . . . . . . . . . . . 41

3.2 .3 Other Forces . . . . . . . . . . . . . . . . . . . . . . . . . . . . . . . . . . . . . . . . . . . .

3.3 Dust Structures and Phase Transitions in Complex Plasmas . . . . . . . . . . . . 47

3.3.1 Structural Ordering of Dust Particles . . . . . . . . . . . . . . . . . 47

3.3.2 Melting and Condensation Transitions of the Dust System . . . . . . . . 49

4 Experimental Setup $\quad 55$

4.1 Complex Plasma Experiment .......................... 55 
4.2 CCD Observation Technique . . . . . . . . . . . . . . . . . . . . 59

4.2 .1 CCD Setup . . . . . . . . . . . . . . . . . . . . . . . 59

4.2 .2 Video Analvsis Software . . . . . . . . . . . . . . . . . . . . 60

4.3 DLS Observation Technique . . . . . . . . . . . . . . . . . . . 62

$4.3 .1 \quad$ DLS Setup . . . . . . . . . . . . . . . . . . . . . 62

4.3 .2 Validation of the DLS Setup . . . . . . . . . . . . . . . 65

4.3 .3 Data Acquisition and Analysis . . . . . . . . . . . . . . 67

$\begin{array}{lll}5 & \text { Results } & \mathbf{7 1}\end{array}$

5.1 The Ballistic Regime and Benchmark of DLS with the CCD Technique . . . . . . 71

5.2 Estimation of the Error of DLS Measurements . . . . . . . . . . . . . . . 78

5.3 Detailed Studies of the Phase Transition . . . . . . . . . . . . . . . . 82

5.3.1 The Thermal Energy during a Phase Transition . . . . . . . . . . . . . . 84

5.3.2 External Bias Potential and Confinement Voltage. . . . . . . . . . . . . . 86

5.3.3 Particle Number Density and Height of the Dust Cloud . . . . . . . . . . 93

5.3.4 Discharge Power and Particle Size . . . . . . . . . . . . . . . . . . 96

5.3.5 Gradual Melting from the Bottom to the Top of the Dust Cloud . . . . . 100

5.3.6 Anisotropv between Horizontal and Vertical Movement . . . . . . . . . 106

5.3.7 Melting Induced by the Increase of the Discharge Powen . . . . . . . . 110

5.3 .8 Summarv . . . . . . . . . . . . . . . . . . . . . . . . . . 110

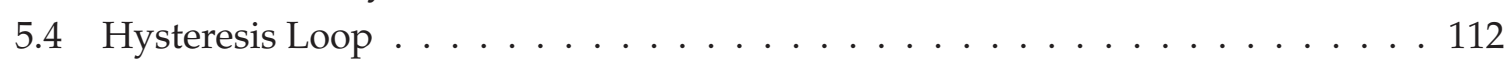

5.5 The Diffusive Regime . . . . . . . . . . . . . . . . . . . . . . . . . . 115

6 Conclusion and Outlook 123

\begin{tabular}{|ll}
\hline Appendix & 129
\end{tabular}

List of Symbols and Abbreviations

$\begin{array}{ll}\text { Bibliography } & 137\end{array}$ 


\section{Chapter 1}

\section{Introduction}

A "dusty" plasma is a plasma containing an additional component of solid or liquid dust particles. The particle diameter normally ranges from a few nanometers up to tens of microns. The dust particles attain a negative or positive charge due to the thermal fluxes of charged plasma particles on the dust grain surface.

The field of dusty plasmas is a well-established part of plasma science for several decades. The main effort has been dedicated to astrophysical science, e.g. planetary rings or planet formation, and to technological applications of dusty plasmas. In plasma technology, the control of growth and behavior of the nano-scaled dust component is important. On the one hand, this is essential to avoid contamination and damage of the product, and on the other hand, the implementation of dust particles in plasma deposited layers is used to manipulate the properties of the layers.

In a "complex" plasma, a stronger coupling between micron size dust grains leads to the formation of ordered structures. These ordered structures, also called "plasma crystals", were discovered in 1994 by Chu and I et al. [1], Hayashi et al. [2], and Thomas et al. [3].

Since then, the interest on complex plasmas has strongly increased. They can be used as a model system to study strong interactions, self-organization phenomena, and phase transitions. The observation of particle streams can give insight into hydrodynamics and the interaction with the plasma environment gives rise to new wave phenomena and plasma instabilities.

A complex plasma can be realized in different types of low-temperature plasma discharges, such as thermal plasmas at atmospheric pressure, DC glow discharges, and UV- and radioactivity induced plasmas [4]. In this work, the dust grains are confined in the plasma-wall sheath of a capacitively coupled radio frequency (RF) discharge.

A fascinating feature of complex plasmas are phase transitions. The ordered structures can be melted by variation of discharge parameters like gas pressure or discharge power. The dust grains attain unexpected high thermal energies that exceed the equilibrium value observed in the ordered state by about three orders of magnitude. This results from the openness of the system and the continuous energy transfer from the plasma environment to the dust component. 
The strong increase of the thermal energy, referred as "anomalous heating", is explained by some theoretical approaches. The anisotropy of the plasma conditions in the plasma-wall sheath leads to instabilities that are responsible for the extreme heating of the dust particle component [5, 6, 7, 8, 9].

The study of phase transition phenomena is quite demanding and thus only a few experiments have been performed until now [10]. Most of them are performed on dust systems with only a few layers. Two additional phase states between the ordered and disordered state are described in these works, the "floe and flow" state, where movement of small crystal parts is observed, and the "vibrational" state, where the dust particles are oscillating on their lattice position [11]. These phase states seem to be characteristic for 2D dust systems.

The picture of the phase transitions of a dust system with a lot of layers is yet unknown [10]. Here, the phase transition reveals another phenomenon that is related to the anisotropy of the plasma-wall sheath. The phase transition does not occur at the same time everywhere in the dust system, but a gradual transition is seen, i.e. a transition front moves vertically through the system.

The great interest on complex plasmas is also based on the unique ability to detect the dust motion on the kinetic level. The dust cloud is illuminated by a sheet of laser light and the single dust particles can be detected using a CCD camera system. This CCD technique is widely used in complex plasma research and gives reliable results for the dust motion in 2D. The extension to 3D is realized successfully in only a few approaches [12].

For example, the stereoscopic technique uses three fixed cameras that detect the dust motion from different perspectives. The video data are combined to obtain the 3D positions and trajectories of the dust particles. This technique is only applicable to small dust systems, because the lens systems of the camera have a limited depth of field.

In the digital holography approach, a reference beam and the waves scattered by the dust grains are superimposed in forward direction on the CCD sensor. This results in a hologram that can be used to reconstruct the particle positions numerically. Since the data processing is quite expensive, this method is also restricted to small particle systems.

All CCD-based techniques are limited according to their temporal and spatial resolution. An increase of the pixel resolution normally results in a lower frame rate of the CCD device. Thus, the examination of phase transitions and the fast particle motion in the melted state is not possible in $3 \mathrm{D}$ with nowadays techniques. The $2 \mathrm{D}$ technique is only capable of measuring in the melted state if expensive, high speed cameras are used.

To overcome the limitations of the imaging diagnostics regarding to the temporal and spatial resolution, the "dynamic light scattering" (DLS) technique is proposed in this work. DLS is well-established in other research fields of biology, chemistry, and physics [13, 14, 15]. The particle system is illuminated by coherent laser light and the scattered light is detected. The movement of the particles along the scattering vector causes fluctuations of the scattered intensity. The temporal analysis of these fluctuations gives insight into the dynamics of the 
scatterers in one dimension. Depending on the ratio of collision length of the scatterers and inverse absolute value of the scattering vector, a diffusive motion or a free motion is observed. In the first case, the diffusion constants can be measured and in the latter case, the average particle velocity can be obtained.

This method is applied on dusty plasmas to obtain diffusion constants and to determine particle sizes of nano-scaled dust particles in [16]. Hurd et al. [17] observed free particle motion for polydisperse dust particles with a diameter below $200 \mathrm{~nm}$ and measured a MaxwellBoltzmann velocity distribution.

Khodataev et al. [18] measured diffusion constants in a thermal complex plasma that contains polydisperse dust grains with diameters of the order of $1 \mu \mathrm{m}$. Here, the system showed comparatively strong coupling, related to a liquid phase state.

The aim of this work can be divided into two parts. First, the applicability of DLS on strongly coupled complex plasmas consisting of large micron sized particles is examined. Here, the particle size and inter-particle distance is comparably larger than in earlier DLS experiments. This requires larger scattering volumes and a new design of the optical components.

It is not clear, which regime of particle movement, i.e. free motion, diffusive motion or a mixture of different motions, has to be expected for a DLS experiment in these systems. Furthermore, it has to be demonstrated that the data are sufficiently accurate to study strongly coupled complex plasmas.

The examination of the melted (weakly coupled) phase state of the particle system is connected to much faster time scales. The requirements on the experimental devices are much higher compared to earlier experiments: A high speed data acquisition with sample times of the order of tens of seconds has to be implemented, which is challenging in terms of data streaming and storage.

A new approach to measure the horizontal and the vertical component of dust particle movement in the 3D system at the same time is proposed. The realization of this DLS experiment, that uses only a single laser beam, has to be proven.

It has to be shown, that the systematic and statistical error of the DLS measurement is reasonable to study the ordered as well as the disordered phase state.

The second part of this work is dedicated to the examination of phase transitions in the three dimensional complex plasma. The DLS technique has to be applied to study the dependency of the phase transition on different experimental parameters such as dust particle density, strength of the confinement potentials, and discharge power.

Another subject is the gradual melting of the dust system, that has never been examined so far. The particle movement in horizontal and vertical direction will be analyzed to compare the heat transport and to study the coupling between the two components.

The observations on phase transitions will be compared to the predictions of theory, in particular to the theoretical description and simulations by Joyce and Ganguli et al. [7, 8, 9]. 
This work is organized as follows: Chapter 2 gives an introduction into the DLS theory. The analysis of the intensity fluctuations is done by temporal autocorrelation of the detected signal. In section 2.1, the formalism of time autocorrelation functions is discussed briefly. The physical background of DLS is presented in section 2.2. This section contains a simplified picture to explain the scattering process and a discussion of the most important experimental realization of the DLS method. The theoretical interpretation of the autocorrelation function is treated for free and diffusive particle motion, and the dynamical variables of interest are derived in section 2.3 .

Chapter 3 gives a short introduction into the theory of complex plasmas. In section 3.1 the fundamental particle charging mechanisms in complex plasmas are discussed. Several different forces act on a dust particle in the plasma-wall sheath. The most important forces responsible for the arrangement of the particles are introduced in section 3.2 . Section 3.3 is dedicated to the dust structures that can be expected in a complex plasma. The condition of strong coupling is treated and the transition to a disordered phase state is discussed. The theoretical description of phase transitions is reviewed giving the basis of the theoretical interpretation of the experimental observations.

Chapter 4 is devoted to the experimental setup. The first section 4.1 describes the experimental realization of the complex plasma in a capacitively coupled RF-discharge. Section 4.2 presents the setup of the CCD observation technique and explains the principles of the video data analysis. In section 4.3, the DLS setup is shown. The validity of the presented DLS setup is examined and an assumption of the DLS theory is justified.

Chapter 5 presents the experimental results of this work. In section 5.1, the nature of particle motion observed with the DLS setup is described. The observations are compared to the results obtained with standard CCD technique. In section 5.2, the error of a DLS experiment is estimated.

The systematic and statistical contributions are discussed for measurements in the ordered and the disordered state. The studies on phase transitions are presented in section 5.3. The impact of experimental parameters like particle number density, confinement voltages, and discharge power on the phase transition is examined. Then, the vertical movement of transition fronts through the dust system is investigated. The phase transitions are analyzed for both components of motion and the relation and coupling between the components are studied. Finally, the melting transition induced by an increase of the discharge power is studied shortly. In simulations, the phase transition is found to show a hysteresis loop between melting and condensation transition.

The section 5.4 investigates, if the predicted hysteresis loop can be observed in a real experiment. In section 5.5, the applicability of DLS to measure diffusion constants in the case of comparably large inter-particle distances is discussed. 


\section{Chapter 2}

\section{Dynamic Light Scattering}

Dynamic light scattering terms methods, where temporal fluctuations of scattered light are measured. The temporal evolution of the intensity of the scattered light is related to the motion of the scatterers. Thus, not only the structure but also the dynamics of the particle system can be examined by light scattering.

The physical mechanism responsible for the fluctuations can be described as a Doppler effect. The Doppler shifts cause a broadened spectrum in the frequency domain which is equivalent to a fluctuating electric field in the time domain. That means, the time analysis of the scattered light can be understood as a spectroscopic measurement in the limit of small line broadening. Nowadays, temporal measurements can resolve particle movements on time scales down to $10^{-8} \mathrm{~s}$. For faster movements, the direct measurement of the broadened power spectrum of the scattered light is possible by means of dispersive media. The lower limit of this spectral approach is a broadening of approximately $10 \mathrm{MHz}$ which corresponds to a time scale of $10^{-7} \mathrm{~s}$. Both methods are complementary techniques in terms of spectral (temporal) resolution.

The analysis of stochastic processes and fluctuating signals is the purpose of time correlation functions. The autocorrelation function, the correlation of the stochastic signal with itself, reveals the characteristic decay time of the fluctuation. The theoretical form of the autocorrelation function gives information about the kind of particle motion that is responsible for the fluctuations.

The derivation of a theoretical model for the decay form of the autocorrelation function is a sophisticated challenge in general. The temporal and spatial correlations of a big number of particles has to be concerned, which is difficult in particular for interacting particles. But if the assumption of independent and identical scatterers holds, the situation simplifies. The theoretical forms for basic types of particle motion like diffusion, ballistic (free) motion, particle rotation or streaming particles can be derived in this case.

DLS methods are widely used, since coherent laser light, fast light detectors, and rapid autocorrelators are available. Plenty of experimental approaches have been developed to confront problems in physics, biology, chemistry, and medicine. The theoretical aspects of DLS were published in the monographs by Berne [13] in 1976, Pecora [14] in 1985, and Borsali [19] 
in 2008. Apart from the fundamental principles, the theoretical models for various physical conditions are treated in these books. A monograph written by Chu [20] emphasizes on the experimental setup of DLS.

This chapter reviews the relevant basic principles and fundamental theories of DLS. In section 2.1, the formalism of correlation functions is introduced. The decay forms relevant for this work are listed with comments according to the corresponding type of particle motion. In section 2.2, light scattering is treated by a simple example of a pair of particles to demonstrate the origin of intensity fluctuations. The equivalence of this picture to spectral broadening through the Doppler effect is evidenced. Different experimental approaches of DLS experiments are introduced and the equivalence between the results of the different methods is discussed. Section 2.3 offers a more intuitive description of the fluctuations in real space. The fluctuations of the scattered intensity are associated with particle density fluctuations. These are represented by the space time correlation function of the particle density. In the last part of this section, the theoretical decay forms of the autocorrelation functions for diffusive and ballistic particle motion are derived.

\subsection{Fluctuations and Time Autocorrelation Functions}

In the theory of noise and stochastic processes, the time correlation formalism is commonly used to describe the temporal relation between two dynamic properties. In a DLS experiment, the scattered intensity is measured and shows stochastic behavior. The autocorrelation function of the scattered intensity reveals the relaxation time or decay time of density fluctuations in the ensemble of scatterers. The decay form of the autocorrelation function is determined by the kind of particle motion that is present.

The formalism of autocorrelation functions and their basic properties are explained in this section. A short overview of theoretical forms of autocorrelation functions relevant for this work and their physical explanations will be given at the end of this section.

\subsubsection{Time Autocorrelation Functions}

To introduce the autocorrelation formalism, a property $A(t)$ that depends on the particle density distribution is assumed. The thermal motion of the particles makes $A(t)$ a stochastic fluctuating function. A measurement of $A(t)$ results in discrete values of a noise pattern as shown in figure 2.1. The time progress is described by the index $j$. The time increment $\Delta t$ is defined by the sampling rate of the measurement.

$A(t)$ fluctuates around the average value $\langle A\rangle$ that is given by

$$
\langle A\rangle:=\lim _{J \rightarrow \infty} \frac{1}{J} \sum_{j=0}^{J} A_{j}
$$




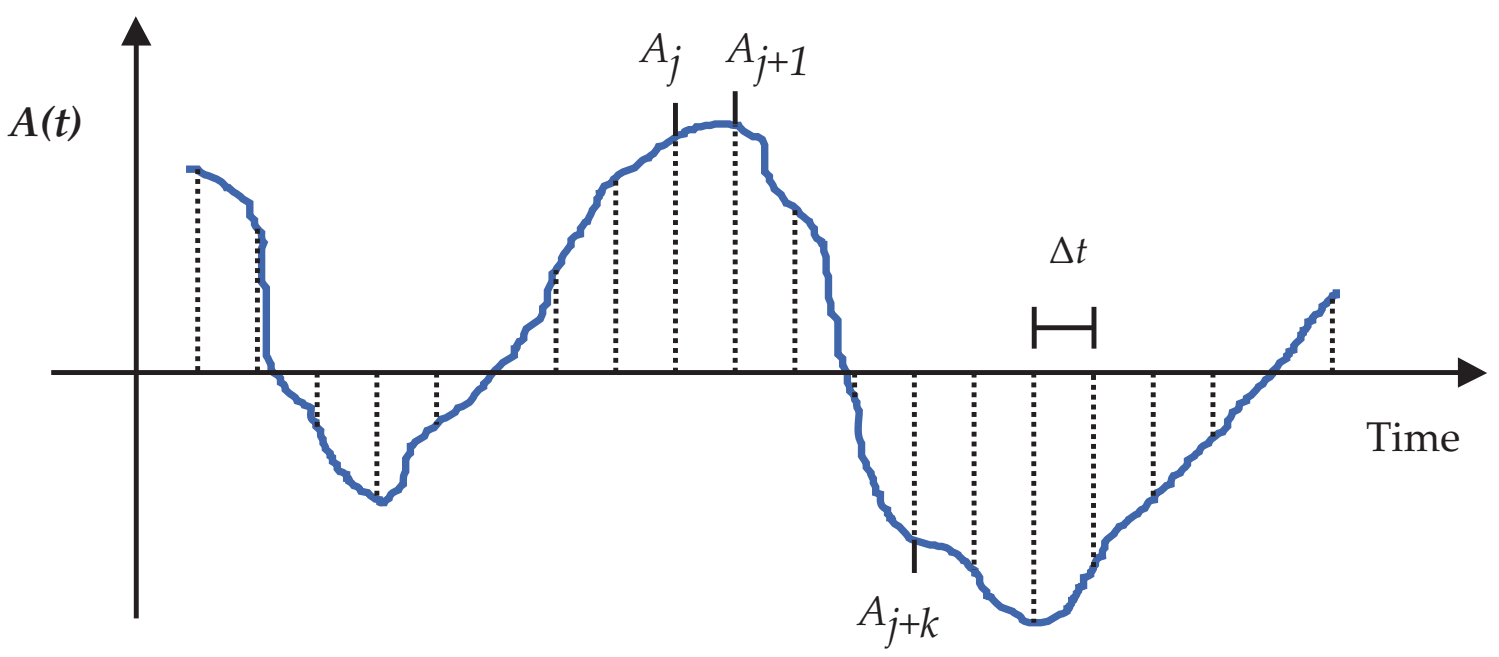

Figure 2.1: Noise pattern of the stochastic function $A(t)$. The data points in a discrete measurement are taken with a time increment $\Delta t$. The correlation between close points $A_{j}$ and $A_{j+1}$ is strong. By increasing the lag time between the points, the correlation gets lost (see the pair $A_{j}$ and $A_{j+k}$ ).

for a discrete property $A(t)$. As pictured in figure 2.1, $A_{j+1}$ comes shortly after $A_{j}$ and both have nearly the same values. This means, they are strongly correlated. By increasing the lag time between two points, their correlation decreases, e.g. $A_{j+k}$ strongly deviates $A_{j}$. The magnitude of correlation between points that are separated by the lag time $\tau$ is described by the autocorrelation function, the temporal cross-correlation of $A(t)$ with itself. The autocorrelation function is defined by

$$
\langle A(0) A(\tau)\rangle_{\text {cont. }}:=\lim _{T \rightarrow \infty} \frac{1}{T} \int_{0}^{T} d t A(t) A(t+\tau)
$$

for a continuous function and

$$
\langle A(0) A(m)\rangle_{\text {discr. }}:=\lim _{J \rightarrow \infty} \frac{1}{J} \sum_{j=0}^{J} A_{j} A_{j+m}
$$

for the discrete case, respectively.

The autocorrelation function is a function of the lag time $\tau$ or $m$ in the discrete case with $\tau=m \Delta t$. For a lag time $\tau=m \Delta t$, the strength of correlation is given by the average of all products from $A_{1} A_{1+m}$ to $A_{J-m} A_{J}$, where $J$ denotes the number of points in the whole data sample. To calculate the whole autocorrelation function, all lag times of interest have to be treated.

The exponential decay in figure 2.2 gives a common example of an autocorrelation function. The characteristic decay time $\tau_{q}$ represents the average time it needs to significantly change the property $A(t)$, i.e. to change it by a factor of $\frac{1}{e}$. Since the property $A(t)$ is related to the 


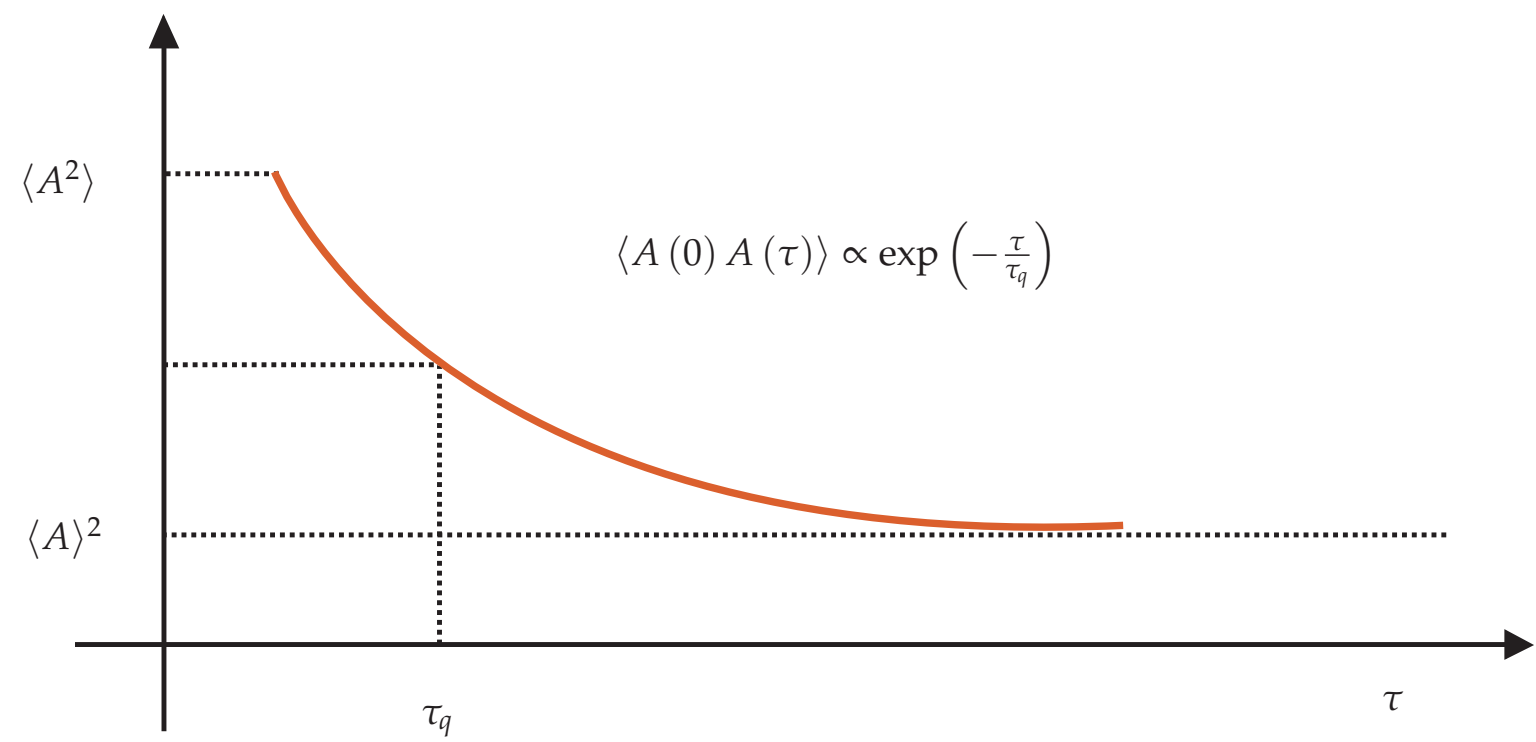

Figure 2.2: Autocorrelation function with an exponential decay form. The decay starts at the initial value $\left\langle A^{2}\right\rangle$ and decreases to $\langle A\rangle^{2}$ in the limit of long times. The decay time $\tau_{q}$ represents the relaxation time of the particle system.

particle positions, this time also stands for the relaxation time of the particle distribution.

The initial value of the autocorrelation function is given by $\langle A(0) A(0)\rangle$. In this case, all summands in equation (2.3) are positive considering the contributions are squares $\sum_{j} A_{j} A_{j}=$ $\sum_{j} A_{j}^{2}$. On the other hand, for other lag times $\tau>0$, negative contributions are possible as illustrated in figure 2.1. This implies the relations

$$
\begin{aligned}
\sum_{j=0}^{J} A_{j}^{2} & \geq \sum_{j=0}^{J} A_{j} A_{j+m} \\
\Leftrightarrow \quad\left\langle A(0)^{2}\right\rangle & \geq\langle A(0) A(\tau)\rangle
\end{aligned}
$$

In the long time limit, the correlation gets lost and the autocorrelation function decays to $\langle A\rangle^{2}$.

$$
\lim _{T \rightarrow \infty}\langle A(0) A(\tau)\rangle=\langle A(0)\rangle\langle A(\tau)\rangle=\langle A\rangle^{2} .
$$

Thus, for a stochastically fluctuating property $A(t)$ the autocorrelation function decays from the maximal value $\left\langle A^{2}\right\rangle$ to the minimum $\langle A\rangle^{2}$.

According to data analysis, it is sometimes advantageous to use the autocorrelation of fluctuations $\langle\delta A(0) \delta A(\tau)\rangle$ of $A(t)$ instead of $\langle A(0) A(\tau)\rangle$. The fluctuation of $A(t)$ is defined as the deviation of the average value.

$$
\delta A(t):=A(t)-\langle A\rangle
$$


Using equation (2.6), we get the autocorrelation function for fluctuations of $A(t)$

$$
\langle\delta A(0) \delta A(\tau)\rangle=\langle A(0) A(\tau)\rangle-\langle A\rangle^{2} .
$$

At zero time $t=0$ we get

$$
\langle\delta A(0) \delta A(0)\rangle=\left\langle A^{2}\right\rangle-\langle A\rangle^{2}=\left\langle\delta A^{2}\right\rangle,
$$

which can be used to normalize the autocorrelation function so that it decays from 1 to zero.

\subsubsection{Common Forms of Autocorrelation Functions}

The decay form of the autocorrelation function represents the kind of motion taking place in the particle system. A short overview about the most important decay forms is given here.

\section{Exponential Decay Form}

$$
\langle A(0) A(\tau)\rangle=a \exp \left(-\frac{\tau}{\tau_{q}}\right)
$$

The exponential decay form can be associated with diffusive motion of the scatterers. The characteristic decay time $\tau_{q}$ can be used to calculate the diffusion constant as will be shown later.

\section{Gaussian Decay Form}

$$
\langle A(0) A(\tau)\rangle=a \exp \left(-\frac{2 \tau^{2}}{\omega_{q}^{2}}\right)
$$

The Gaussian form occurs in many cases. This form is typically measured for particles that undergo ballistic (free) motion with a Maxwell-Boltzmann velocity distribution. The decay time can be used to calculate the average velocity of the particles and the autocorrelation function can be transformed into the velocity distribution of the particles [21].

\section{Sum of Decay Forms}

$$
\langle A(0) A(\tau)\rangle=\sum_{i} a_{i} \exp \left(-\frac{\tau}{\tau_{i}}\right)
$$

A sum of decay forms is found if several independent physical processes take place at the same time. Each process is represented by its own decay and they can be analyzed separately if the decay times are sufficiently different.

\section{Oscillating Decay Form}

$$
\langle A(0) A(\tau)\rangle=a \cos \left(\omega_{o s c} t\right) \exp \left(-\frac{\tau}{\tau_{q}}\right)
$$


Periodic processes cause the autocorrelation function to oscillate with the same frequency $\omega_{\text {osc }}$ like the process. For example any wave propagation through the background media or the particle system itself would appear as a cosine term in the autocorrelation function. Another origin of cosine terms are constant particle motions in one direction. These streaming particles cause a cosine term with a frequency depending on the stream direction and velocity.

\subsection{Basics of Dynamic Light Scattering}

To understand the connection between intensity fluctuations of scattered light on the one hand and the movement of the scatterers on the other hand, the fundamental formalism of light scattering of coherent light is discussed in the first part section 2.2.1.

In the following section 2.2.2, two common experimental approaches in DLS are treated. Firstly, optical mixing experiments are introduced which take place in the temporal domain. Fluctuations of the scattered intensity are measured directly with high temporal resolution detectors.

Secondly, the filter technique that measures in the spectral domain is discussed shortly. The Fourier components of the scattered light are analyzed by dispersive media such as prisms, gratings or interferometers. The Doppler effect causes line broadening that gives insight into the dynamics of the system. As will be shown, the two methods are basically equivalent in terms of the available spectroscopic information. The fluctuations as well as the line broadening can be explained by the Doppler effect.

Section 2.2.3 deals with the scattering vector $\mathbf{q}$, one of the central experimental parameters in DLS. It determines the temporal and spatial scales of the measurement. Hence, the physical processes of interest require an appropriate choice of the scattering vector.

The concept of "coherence area" (section 2.2.4) is fundamental in DLS to ensure sufficient spatial coherence of the scattered light. The detection of too large solid angles of scattered light leads to averaging of statistically independent (or spatially incoherent) beams of scattered light. In this case, the amplitude of the fluctuations is decreased or the fluctuation even vanishes. Hence, the size of the scattering volume as well as the detector area have to be carefully adjusted to get an autocorrelation function with a sufficient amplitude.

\subsubsection{Principles of Dynamic Light Scattering}

To gain a deeper understanding of the connection between movement of particles and intensity fluctuations, a simplified system of two particles is considered here. Figure 2.3 (a) depicts the scattering of an incident plane wave of laser radiation with wave vector $\mathbf{k}_{\mathbf{i}}$ on two spherical particles into the direction of $\mathbf{k}_{\mathbf{f}}$. The detected signal is determined by superposition of the scattered electric fields of the two particles at positions $\mathbf{r}_{\mathbf{i}}$ and $\mathbf{r}_{\mathbf{j}}$. The path difference for the incident wave is $\Delta r \sin \left(\phi_{p h}\right)$. The associated phase shift is given 
(a)

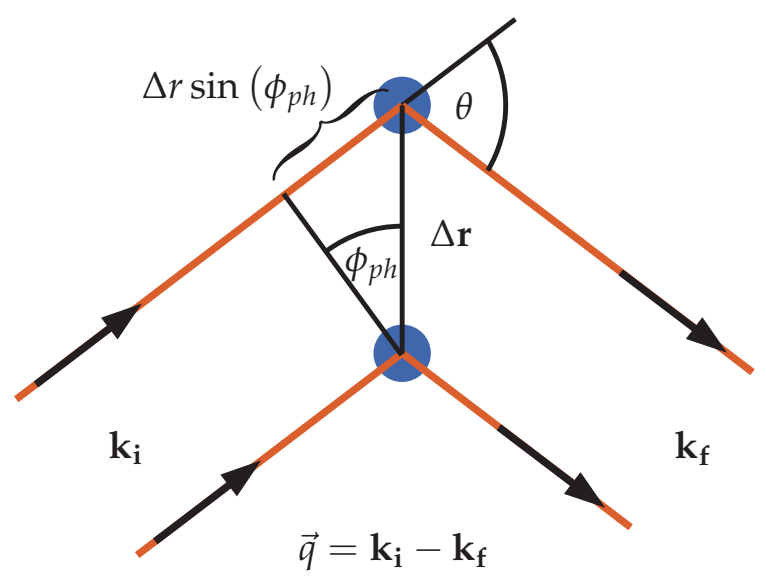

(b)

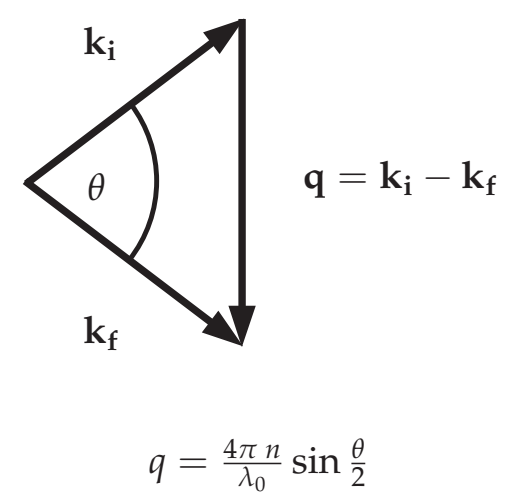

Figure 2.3: (a) Scattering on a pair of particles with distance $\Delta \mathbf{r}=\mathbf{r}_{\mathbf{i}}-\mathbf{r}_{\mathbf{j}}$. The incident wave with wave vector $\mathbf{k}_{\mathbf{i}}$ is scattered quasi-elastic under the angle $\theta$ into the direction of $\mathbf{k}_{\mathbf{f}}$. The path difference between the incident beams is given by $\Delta r \sin \left(\phi_{p h}\right)$. (b) Vector diagram of the scattering vector $\mathbf{q}=$ $\mathbf{k}_{\mathbf{i}}-\mathbf{k}_{\mathbf{f}}$.

by $\left(2 \pi \Delta r \sin \left(\phi_{p h}\right)\right) / \lambda_{0}=\Delta \mathbf{r} \cdot \mathbf{k}_{\mathbf{i}}$, where $\lambda_{0}$ is the wavelength of the incident light in vacuo. Following the same arguments, the scattered wave has a phase shift of $-\Delta \mathbf{r} \cdot \mathbf{k}_{\mathbf{f}}$ so that the total phase shift between the waves is $\left(\mathbf{k}_{\mathbf{i}}-\mathbf{k}_{\mathbf{f}}\right) \cdot \Delta \mathbf{r}=\mathbf{q} \cdot \Delta \mathbf{r}$, where $\mathbf{q}:=\mathbf{k}_{\mathbf{i}}-\mathbf{k}_{\mathbf{f}}$ denotes the scattering vector as shown in figure 2.3 (b).

The wavelength can usually be assumed to be constant during the scattering process (quasielastic scattering) so that

$$
\left|\mathbf{k}_{\mathbf{i}}\right| \cong\left|\mathbf{k}_{\mathbf{f}}\right|
$$

applies. In this case, the scattering vector $\mathbf{q}$ can be transformed according to the law of cosines to an expression that basically depends on the scattering angle $\theta$ for given wavelength $\lambda_{0}$ and refractive index $n$.

$$
\begin{aligned}
|\mathbf{q}|^{2} & =\left|\mathbf{k}_{\mathbf{i}}-\mathbf{k}_{\mathbf{f}}\right|^{2}=k_{i}^{2}+k_{f}^{2}-2 \mathbf{k}_{\mathbf{i}} \cdot \mathbf{k}_{\mathbf{f}} \\
& \cong 2 k_{i}^{2}-2 k_{i}^{2} \cos \theta=4 k_{i}^{2} \sin ^{2} \frac{\theta}{2} \\
\Leftrightarrow \quad q & \cong 2 k_{i} \sin \frac{\theta}{2}=\frac{4 \pi n}{\lambda_{0}} \sin \frac{\theta}{2}
\end{aligned}
$$

The amplitude of the scattered intensity is determined by the phase shift between both waves. This is described by the phase factor of the two particles

$$
\exp \left(i \mathbf{q} \cdot\left(\mathbf{r}_{\mathbf{i}}-\mathbf{r}_{\mathbf{j}}\right)\right)
$$

The phase factor contains information on the dynamics of the particle system and therefore 
plays a central role in DLS.

For an ensemble of particles, the scattered electric field results from superposition of the contributions of all scatterers

$$
E_{S}(\mathbf{q}, t):=\sum_{j=0}^{N} a_{j} \exp \left(i \mathbf{q} \cdot \mathbf{r}_{\mathbf{j}}(t)\right) \exp \left(-i \omega_{i} t\right)
$$

Here, $N$ is the number of particles in the ensemble, $\mathbf{q}$ is the scattering vector, $\mathbf{r}_{\mathbf{j}}$ the position of the scatterers, $a_{j}$ is the amplitude of the scattered electric field, and $\omega_{i}$ is the frequency of the incident light.

The total scattered intensity $I(\mathbf{q}, t)$ is the sum over the scattered intensities of all pairs of particles.

$$
I(\mathbf{q}, t):=\sum_{i=1}^{N} \sum_{j=1}^{N} a_{i} a_{j} \exp \left[i \mathbf{q} \cdot\left(\mathbf{r}_{\mathbf{i}}(t)-\mathbf{r}_{\mathbf{j}}(t)\right)\right]
$$

In principle, the amplitudes of the scattered electric field $a_{i}$ and $a_{j}$ can be time-dependent too, but for spherical and isotropic scatterers this can be neglected.

Equation (2.17) clearly demonstrates the connection between scattered intensity $I(\mathbf{q}, t)$ and particle positions $\mathbf{r}_{\mathbf{i}}$ and $\mathbf{r}_{\mathbf{j}}$. The scattered intensity changes on same time scales as the particle distribution which is used in DLS to analyze the dynamics of the scatterers.

Two other important properties of the DLS technique are revealed by equation (2.17). Firstly, only particle movements along the scattering vector $\mathbf{q}$ change the phase factor and therefore the scattered intensity. Hence, DLS is per definition a one-dimensional diagnostic. Secondly, the distance between a pair of particles has to change by a value of $q^{-1}$ to significantly change the phase factor in equation (2.17). The inverse absolute value of the scattering vector $q^{-1}$ therefore defines the length and time scale of the measurement. The consequences of this are discussed in more detail in section 2.2.3

\subsubsection{Dynamic Light Scattering Techniques}

\section{Optical Mixing Techniques}

In an optical mixing experiment, the intensity fluctuations are analyzed in the temporal domain. The scattered intensity is directly acquired by means of fast photon detectors.

There are two major operation modes used in a DLS setup, the heterodyne method (figure 2.4 (a)) and the homodyne method (figure 2.4 (b)). In the heterodyne method, a portion of unscattered light (a local oscillator) is superimposed to the scattered light, whereas the homodyne method detects only the scattered laser light 1 .

In both cases, a square-law detector like a photomultiplier is used, so that the detected

\footnotetext{
${ }^{1}$ Some textbooks use a different notation. There, the detection of the scattered intensity only is called self-beating spectroscopy. A mixing with a local oscillator with the same frequency as the incident light is called homodyne technique and a mixing with a different frequency is denoted as heterodyne technique.
} 
(a) heterodyne setup

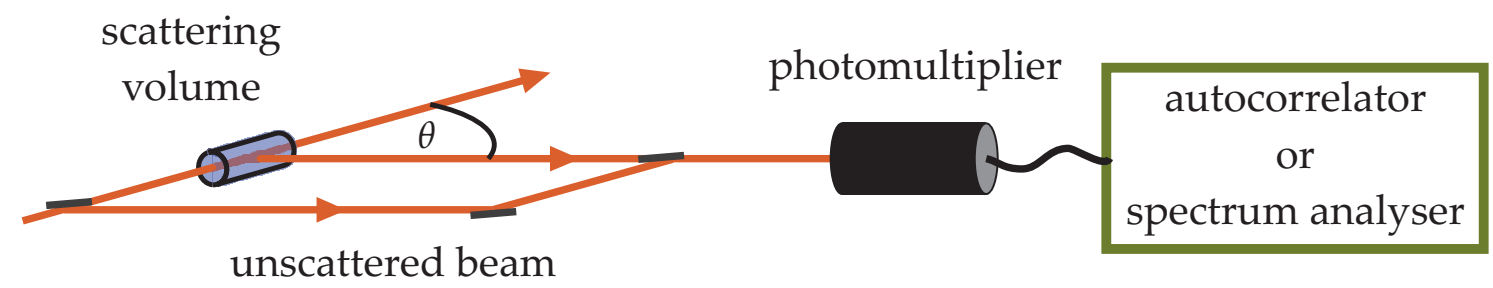

(b) homodyne setup

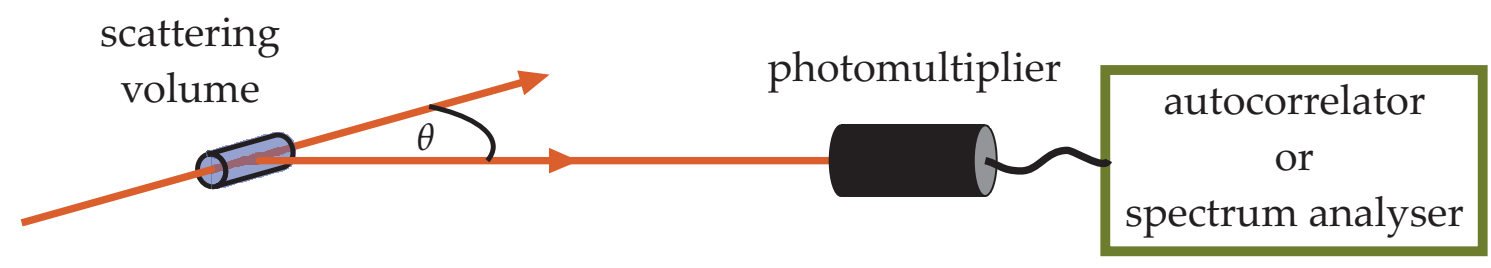

Figure 2.4: Basic setup for optical mixing experiments. The scattered laser light is detected by a fast photomultiplier under an angle $\theta$. (a) In the heterodyne setup, a portion of unscattered light is mixed with the scattered light. (b) In the homodyne setup, only the pure scattered light is measured. The fluctuating signal is analyzed by means of autocorrelators or spectrum analyzers.

signal is proportional to the square of the scattered electric field that reaches the detector $s(t) \propto\left|E_{S}(t)\right|^{2}$. The signal $s(t)$ is used to calculate the time autocorrelation function

$$
\langle s(0) s(t)\rangle \propto\left\langle\left|E_{S}(0)\right|^{2}\left|E_{S}(t)\right|^{2}\right\rangle .
$$

The following discussion about the autocorrelation function $\langle s(0) s(t)\rangle$ for the homodyne and heterodyne case follows the textbook of Berne et al. [13]. First, two autocorrelation functions of the scattered electric field have to be introduced

$$
\begin{aligned}
I_{\text {het }}(t) & :=\left\langle E_{S}^{*}(0) E_{S}(t)\right\rangle \\
I_{\text {hom }}(t) & :=\left\langle\left|E_{S}(0)\right|^{2}\left|E_{S}(t)\right|^{2}\right\rangle,
\end{aligned}
$$

where $E_{S}(t)$ is the scattered electric field (equation (2.16)).

In the homodyne case, only the scattered electric field $E_{S}(t)$ reaches the detector. Thus, the autocorrelation function of the detected signal (equation (2.18)) is proportional to $I_{\text {hom }}(t)$ (equation (2.20)

$$
\langle s(0) s(t)\rangle \propto I_{\text {hom }}(t) .
$$

$I_{\text {hom }}(t)$ is also called the homodyne correlation function.

For the heterodyne technique, the electric field of the local oscillator $E_{L O}(t)$ has to be con- 
sidered in equation (2.18) and the situation becomes more complex.

$$
\langle s(0) s(t)\rangle \propto\left\langle\left|E_{L O}(0)+E_{S}(0)\right|^{2}\left|E_{L O}(t)+E_{S}(t)\right|^{2}\right\rangle
$$

This expression can be simplified using the following approximations:

- The amplitude of the local oscillator is much bigger than the amplitude of the scattered field. $\left|E_{L O}(t)\right| \gg\left|E_{S}(t)\right|$

- Fluctuations of the local oscillator are negligible.

- The electric field of the local oscillator $E_{L O}(t)$ and the scattered electric field $E_{S}(t)$ are statistically independent. $\left\langle I_{L O} I_{S}(t)\right\rangle=\left\langle I_{L O}\right\rangle\left\langle I_{S}(t)\right\rangle$.

The remaining term is

$$
\langle s(0) s(t)\rangle \propto\left[I_{L O}^{2}+2 I_{L O} \operatorname{Re} I_{h e t}(t)\right],
$$

where Re $I_{\text {het }}(t)$ is the real part of $I_{\text {het }}(t)$ (equation (2.19)). Re $I_{\text {het }}(t)$ is sometimes called the "heterodyne correlation function". The background of the heterodyne correlation function is defined by the intensity of the local oscillator $I_{L O}$. The autocorrelation functions measured in a homodyne (equation (2.21) ) and heterodyne (equation (2.23) ) experiment are generally different. In some situations the heterodyne correlation function $I_{\text {het }}(t)$ contains more information than the homodyne correlation function $I_{\text {hom }}(t)$ as it is the case for dilute suspensions of macromolecules ([13], section 5.5).

Under certain conditions, the heterodyne and the homodyne correlation function can be connected by a simple relation.

$$
I_{\text {hom }}(t)=\left|I_{\text {het }}(0)\right|^{2}+\left|I_{\text {het }}(t)\right|^{2}
$$

This equation is known as the "Siegert relation" (see [20]). This relation only holds if the scattered field is distributed over the scattering volume according to a Gaussian distribution. That means, the scattering volume can be divided into a large number of subregions and the scattered intensities of these subregions are statistically independent.

To fulfill this assumption might be difficult for systems with long spatial correlations, where the subregions are not statistically independent or for very dilute systems with only a small number of particles. Most theoretical approaches for the particle motion deal with the heterodyne autocorrelation function $I_{\text {het }}(t)$. That means, if the Siegert relation does not hold, a prediction of the homodyne autocorrelation function $I_{\text {hom }}(t)$ is rather difficult and a heterodyne experiment has to be preferred.

\section{Filter Technique}

In contrast to an optical mixing experiment, the filter technique as shown in figure 2.5 is a 
filter technique

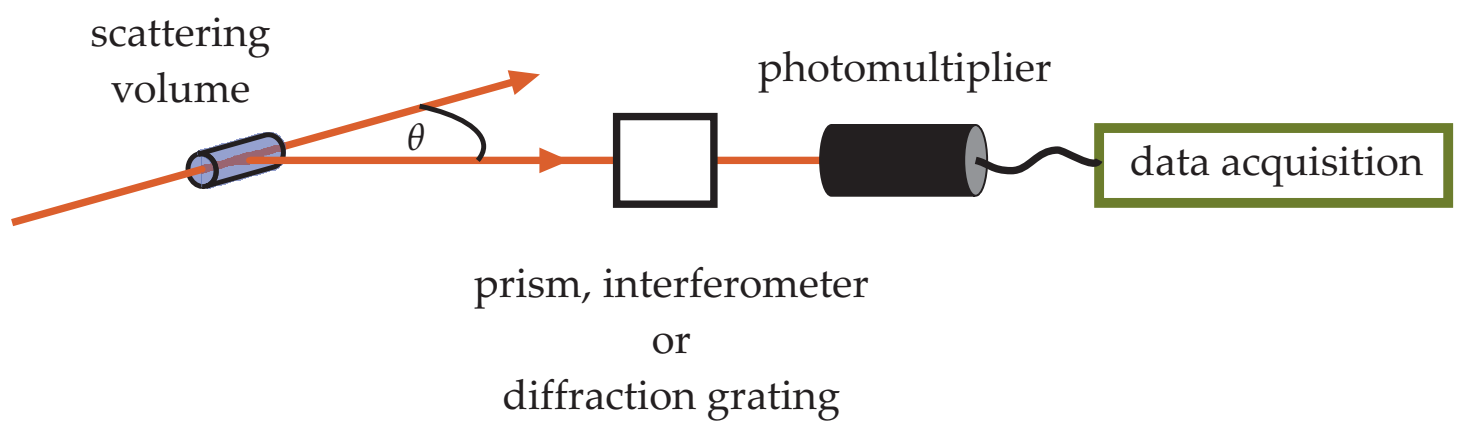

Figure 2.5: Basic setup for a filter experiment. The incident laser beam is scattered by the scattering volume under an angle $\theta$. A certain spectral component is chosen by a dispersive element like a prism, grating or interferometer. The selected component is detected by a square-law detector and analyzed by a personal computer. By scanning over all components, the whole power spectrum can be measured.

spectral measurement. A narrow range of the power spectrum of the scattered light is measured directly. The whole power spectrum can be obtained by scanning techniques.

This technique is appropriate for very fast particle movements that cannot be resolved in the temporal regime directly. As a rule of thumb, the filter technique basing on Fabry-Perot interferometry can resolve dynamical processes on time scales between $10^{-7} \mathrm{~s}$ and $10^{-11} \mathrm{~s}$. For faster time scales a diffraction grating can be used.

The acquired power spectrum reveals line broadening which corresponds to the particle movement and therefore comprises the dynamic information of interest. The fundamental physical process to understand this broadening is the Doppler effect. The frequency of the scattered light $\omega_{f}$ is shifted with respect to the initial frequency $\omega_{i}$ through the Doppler effect as

$$
\omega_{f}=\omega_{i} \frac{\left(1-\frac{\mathbf{e}_{\mathbf{i}} \cdot \mathbf{v}}{c}\right)}{\left(1-\frac{\mathbf{e}_{\mathbf{f}} \cdot \mathbf{v}}{c}\right)},
$$

where $\mathbf{e}_{\mathbf{i}}$ and $\mathbf{e}_{\mathbf{f}}$ are the unit vectors into the direction of the incident laser beam and the detector and $\mathbf{v}$ denotes the particle velocity.

The change of frequency $\Delta \omega$ can be written as a function of the scattering vector $\mathbf{q}$ and the particle velocity $\mathbf{v}$ [22].

$$
\Delta \omega=\omega_{f}-\omega_{i}=\left(\mathbf{k}_{\mathbf{f}}-\mathbf{k}_{\mathbf{i}}\right) \cdot \mathbf{v}=\mathbf{q} \cdot \mathbf{v}
$$

\section{Relation Between Optical Mixing and Filter Technique}

It has to be emphasized that the optical mixing technique as well as the filter technique are complementary approaches. The interpretation of the fluctuations, as shown in section 2.2.1. is based on time-dependent phase shifts between pairs of particles. But, the origin of the fluctuations can be understood by the Doppler mechanism as well. If two particles move 
filter

\section{optical mixing}

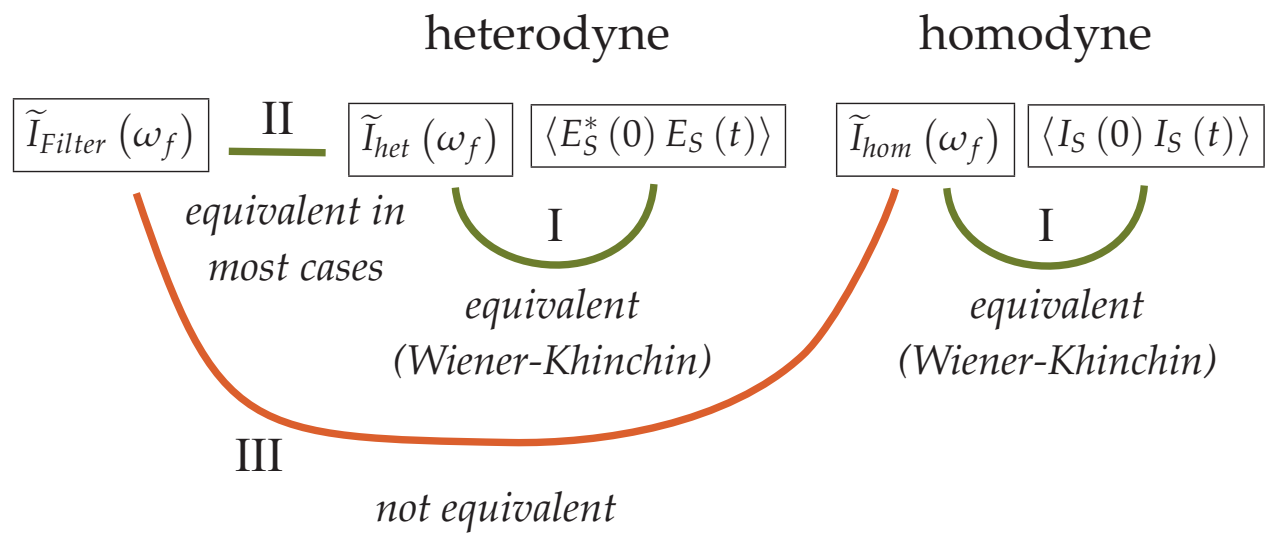

Figure 2.6: Connections between the results of different DLS methods. I) In an optical mixing experiment, the Wiener-Khinchin theorem ensures equivalence between the autocorrelation function and the power spectrum of the fluctuating signal. II) The power spectrum of a heterodyne measurement $\widetilde{I}_{\text {het }}\left(\omega_{f}\right)$ is in most cases equivalent to the power spectrum of a filter experiment $\widetilde{I}_{\text {Filter }}\left(\omega_{f}\right)$. III) This is not valid for the homodyne power spectrum $\widetilde{I}_{\text {hom }}\left(\omega_{f}\right)$.

with different velocities along $\mathbf{q}$, there will be a frequency shift (equation (2.26) between the scattered waves due to the Doppler effect. This modulates the scattered intensity with the resulting beat frequency. A certain distribution of velocities leads to a distribution of beat frequencies (broadened spectrum) which results in a stochastic fluctuation of the scattered intensity.

The measurement by optical mixing in the temporal domain or by the filter technique in the frequency domain should not affect the content of information available. But as shown in figure 2.6, an equivalence between the methods is not always given.

In the first case of optical mixing (I in figure 2.6), it is absolutely equivalent if the fluctuating signal $s(t)$ is treated by means of autocorrelation function or a spectral Fourier analysis. The autocorrelation and the power spectrum are Fourier transforms of each other which is expressed by the Wiener-Khinchin theorem

$$
\widetilde{I}_{s}\left(\omega_{f}\right)=\frac{1}{2 \pi} \int_{-\infty}^{\infty} d t \exp \left(-i \omega_{f} t\right)\left\langle s^{*}(0) s(t)\right\rangle .
$$

The connection between heterodyne optical mixing experiments and filter experiments is described by II in figure 2.6. Here, the Fourier transformation of (equation (2.27) ) of the heterodyne autocorrelation function $\left\langle E_{S}^{*}(0) E_{S}(t)\right\rangle$ results in a power spectrum $\widetilde{I}_{\text {het }}\left(\omega_{f}\right)$ that 
(a)

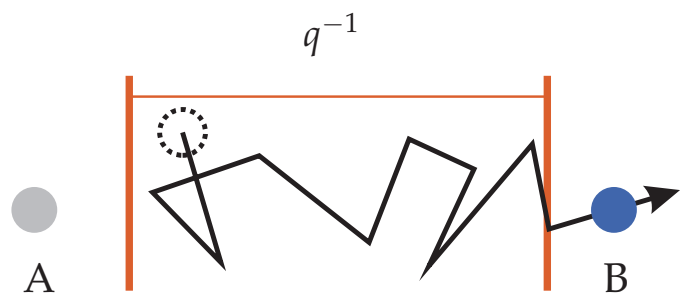

(b)

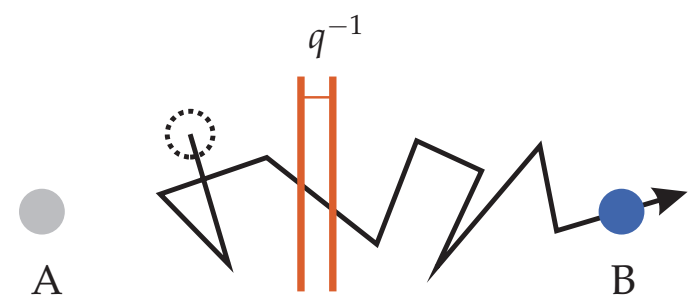

Figure 2.7: The impact of the absolute inverse scattering vector $q^{-1}$ on the result of a DLS experiment. One particle A is fixed and a second particle B moves to the right under several collisions. (a) If $q^{-1}$ is large relative to the collision length (small scattering angle), the fluctuations will represent diffusive motion. (b) The very same movement is probed with a small length $q^{-1}$ and the fluctuation decays on faster time scales. Linear trajectories are observed along $q^{-1}$ and the measured fluctuations correspond to ballistic (free) motion.

depends only on the real part of $I_{\text {het }}(t)$ (see equation (2.23)

$$
\widetilde{I}_{h e t}\left(\omega_{f}\right) \equiv \frac{1}{2 \pi} \int_{-\infty}^{\infty} \mathrm{d} t e^{-i \omega_{f} t} \operatorname{Re} I_{h e t}(t) .
$$

It follows, the power spectrum $\widetilde{I}_{\text {het }}\left(\omega_{f}\right)$ is equivalent to the one obtained in a filter experiment $\widetilde{I}_{\text {Filter }}\left(\omega_{f}\right)$ as long as $I_{\text {het }}(t)=\left\langle E_{S}^{*}(0) E_{S}(t)\right\rangle$ is a real function of time, which is true in most cases. An example for a complex heterodyne autocorrelation function is the case of particles with a uniform motion [13].

The third type of connection (III in figure 2.6) is between homodyne optical mixing experiments and filter experiments. Here, no general equivalence can be found between the spectra of optical mixing and filter method.

\subsubsection{The Scattering Vector $q$}

As mentioned at the end of section 2.2.1, the inverse absolute value of the scattering vector $q^{-1}$ defines the length and time scale on that the particle movement is probed (see equation (2.17)). As a rule of thumb, a small $q^{-1}$ probes rapid motion on small length scales and large $q^{-1}$ probes the long time regime and big length scales. The length scale $q^{-1}=$ $\lambda_{0} /(4 \pi n \sin (\theta / 2))$ is a function of the scattering angle $\theta$, wavelength of the incident light $\lambda_{0}$ and the refractive index $n$ of the background media.

To demonstrate the impact of $q^{-1}$, again a simple situation with two particles is assumed as shown in figure 2.7. The particle A is fixed and a second particle B is moving to the right and suffers several collisions on its way. In the first case (figure 2.7 (a)), a small scattering angle $\theta$ is chosen, which results in a large value $q^{-1}$. The particle B has to move the distance $q^{-1}$ to cause a significant change in the scattered intensity. The nature of this fluctuation is determined by the kind of motion taking place on this distance. Thus, in case (a) the fluctuations will represent a diffusive particle motion. The autocorrelation function according to diffusive 
(a)

source

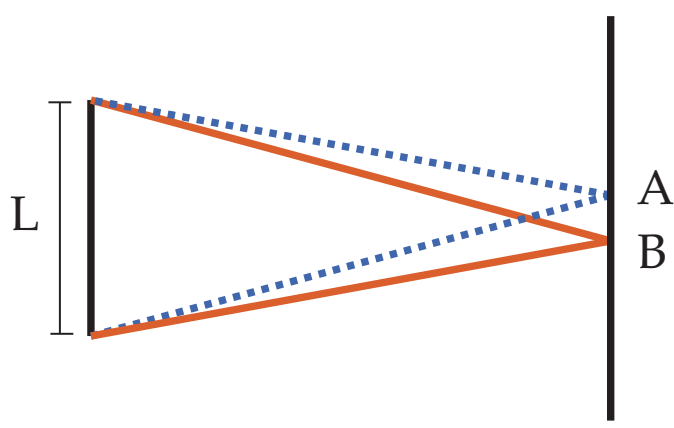

(b)

source screen

Figure 2.8: To elucidate the coherence of the scattered electric field at two different points $A$ and $B$ on the observation screen, a one-dimensional source of incoherent light $L$ is assumed. (a) If the distance between the points is small, all path lengths of light are comparable and the scattered light at $A$ and $B$ is mostly coherent. (b) For large distances between $A$ and $B$, the path lengths become different and the coherence gets lost. The scattered electric fields at $A$ and $B$ are statistically independent.

motion decays exponentially.

In the second case (figure 2.7 (b)), the very same motion is probed with a large scattering angle $\theta$, i.e. a small $q^{-1}$. The decay time of the fluctuations is of course smaller, and the type of fluctuation has changed because the type of motion on the distance $q^{-1}$ is different. The trajectories along $q^{-1}$ are linear, so the particle moves ballistically or freely. A Gaussian decay form is related to this kind of motion.

This demonstrates, the choice of $q^{-1}$ in ratio to the mean free path $l_{m f}$ of collisions between scatterers defines the form of the autocorrelation function and therefore the kind of dynamic parameter that is studied. To examine particle interactions of charged particles, the length scale $q^{-1}$ has to be larger than the interaction length or of the size of the lattice constant for crystalline structures. For length scales containing a lot of particles, hydromagnetic modes and global fluid motions can be studied on long time scales.

\subsubsection{The Coherence Area}

Equation (2.17) is an expression for the scattered intensity at a point in the detection plane. In a real experiment, all the light that impinges within a detection area $A_{D}$ is detected. That means, not only light with a defined scattering angle $\theta$ is detected but light from a specific solid angle reaches the detector. It turns out that the scattered light is only spatially coherent within a certain area, the so-called "coherence area". The scattered intensity in different coherence areas fluctuates statistically independent. Hence, if a large number of coherence areas is detected, the fluctuations are averaged and a mean intensity is measured. This refers to static light scattering. 
Figure 2.9: General scattering geometry for DLS. The incident light with wave vector $k_{i}$ is scattered and detected under an azimuth angle $\theta_{a z}$ and an inclination angle $\theta_{i n c}$ into the direction of $\mathbf{k}_{\mathbf{f}}$. The scattering volume is defined as a cube with dimensions of $L_{x}, L_{y}$, and $L_{z}$.

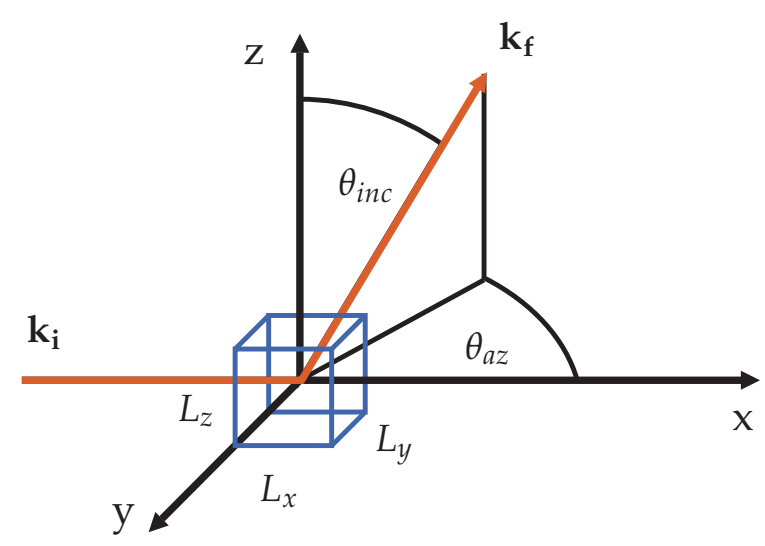

The concept of "coherence area" is elucidated in the following for the one-dimensional case according to the textbook of Pecora et al. [14]. Figure 2.8 shows a source of incoherent light with linear dimension $L$. This represents the particle system.

The spatial coherence of the emitted light at the points $A$ and $B$ on the observation screen depends on the distance between the points. In figure 2.8 (a) $A$ and $B$ are very close to each other and all path lengths of the light to the points are nearly identical. Each source contributes nearly with the same phase shift to $A$ and $B$ and therefore the incoherence of the emitted light does not affect the spatial coherence. In figure 2.8 (b), the path lengths to $A$ and $B$ are different, i.e. the contributions to $A$ have different phase shifts than to $B$. The incoherence of the emitted light results in statistically independent fluctuations in the scattered intensities in $A$ and $B$.

The coherence length $l_{c}$ describes the decay length of the spatial correlation function $\langle E(A) E(B)\rangle$. Note, this length also depends on the extension of the source. The points $A$ and $B$ have to be closer to obtain spatial coherence for a larger $L$.

Within a coherence length, the scattered electric fields are sufficiently in phase and therefore "partially" coherent. At a distance $l_{c}$, the detected intensities are on average distinguished by a factor of $e$. An expression for the coherence area according to a cubic scattering volume of $L_{x} \cdot L_{y} \cdot L_{z}$ (see figure 2.9) is derived by Lastovka in [23]:

$$
A_{c o h}:=\frac{R^{2} \lambda_{0}^{2}}{L_{y} L_{z} \sin \theta_{i n c} \cos \theta_{a z}+L_{x} L_{z} \sin \theta_{i n c} \sin \theta_{a z}+L_{x} L_{y} \cos \theta_{i n c}},
$$

where $\lambda_{0}$ is the wavelength of the incident beam, $L_{x}, L_{y}$ and $L_{z}$ are the dimensions of the scattering volume, and $R$ is the distance between scattering volume and detector.

\subsubsection{Number Fluctuations}

Another source of fluctuations has been neglected until now. As illustrated in figure 2.10, the change of the number density of scatterers in the scattering volume gives rise to intensity fluc- 


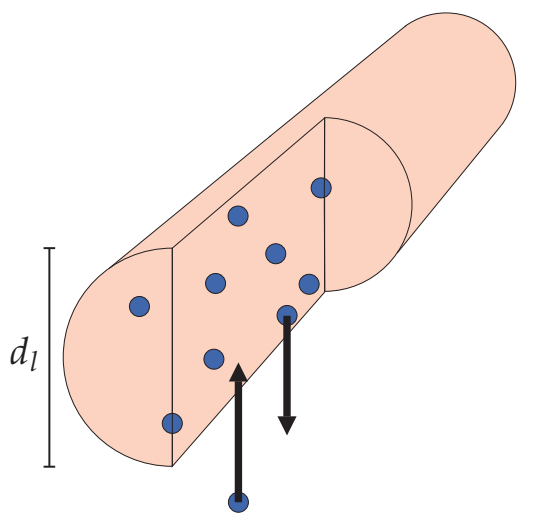

Figure 2.10: The scattered intensity fluctuates due to particles that leave or enter the scattering volume. The characteristic length for number fluctuations is given by the diameter $d_{l}$ of the laser beam and aperture size of the detection system, respectively.

tuations on a different time scale than the interference fluctuations. The characteristic length scale for number fluctuations can be estimated by the diameter of the laser beam $d_{l}$. Considering that $d_{l}$ is much bigger than the characteristic length $q^{-1}$ for interference fluctuations, both decays can normally be separated in time. Number fluctuations are in particular important for dilute systems of scatterers, because the fluctuation of the particle number $\Delta N / N$ scales as $1 / \sqrt{N}$.

\subsection{Theoretical Forms}

The autocorrelation functions $I_{\text {het }}(t)$ and $I_{\text {hom }}(t)$ as introduced in section 2.2.2 are functions of the reciprocal domain because they are functions of the scattering vector $\mathbf{q}$. The formulation in real space rather than in reciprocal space may be more intuitive. In section 2.3.1, the autocorrelation functions will be connected to an equivalent probability function in time and space for the particle density.

The in general complicated situation of temporal and spatial correlations of a huge number of scatterers can be simplified, if identical and independent scatterers are assumed. For this case, the theoretical forms for diffusive motion and free particle motion will be derived in section 2.3.2.

\subsubsection{Space Time Correlation Function of the Particle Density}

In the following, the space time correlation function for identical point-like particles is discussed [19]. For identical particles, the amplitude of the electric field scattered by a single particle is the same for all particles $a_{j} \equiv a$. Hence, the entire scattered electric field (equation (2.16)) is proportional to the sum over all phase factors

$$
\Psi(\mathbf{q}, t):=\sum_{j=0}^{N} \exp \left(i \mathbf{q} \cdot \mathbf{r}_{\mathbf{j}}(t)\right) .
$$


In analogy to section 2.2.2, the homodyne and heterodyne autocorrelation functions of the phase factor are defined for the following derivation

$$
\begin{aligned}
F_{\text {het }}(\mathbf{q}, t) & :=\left\langle\Psi^{*}(\mathbf{q}, t) \Psi(\mathbf{q}, 0)\right\rangle \\
F_{\text {hom }}(\mathbf{q}, t) & :=\left\langle\left|\Psi^{*}(\mathbf{q}, t)\right|^{2}|\Psi(\mathbf{q}, 0)|^{2}\right\rangle .
\end{aligned}
$$

$F_{\text {het }}(\mathbf{q}, t)$ and $F_{\text {hom }}(\mathbf{q}, t)$ give the connection between experiment and theory.

The relation to the real space is revealed by a Fourier transformation of the phase factor (equation (2.30). This leads to a sum over Dirac delta functions $\delta$.

$$
\int d^{3} q\left[\sum_{i=0}^{N} \exp \left(i \mathbf{q} \cdot \mathbf{r}_{\mathbf{i}}\right)\right] \exp (i \mathbf{q} \cdot \mathbf{r})=\sum_{i=0}^{N} \delta\left(\mathbf{r}-\mathbf{r}_{i}(t)\right) .
$$

The expression of the right hand side is the definition of the particle density distribution by the aid of the Dirac delta function $\delta(\mathbf{r})$.

$$
\rho(\mathbf{r}, t):=\sum_{i=0}^{N} \delta\left(\mathbf{r}-\mathbf{r}_{i}(t)\right) .
$$

This clearly shows that the phase factor and the particle density carry analogue information about the particle dynamics but in reciprocal spaces. Note, by choosing a certain scattering vector $\mathbf{q}$, only the q-th spatial Fourier component of the particle density fluctuation is probed as already elucidated in section 2.2.3.

The temporal and spatial evolution of the particle density distribution $\rho(\mathbf{r}, t)$ is analyzed by the time space autocorrelation function

$$
\left\langle\rho(\mathbf{r}, t) \rho\left(\mathbf{r}^{\prime}, 0\right)\right\rangle:=\left\langle\sum_{i=1}^{N} \sum_{j=1}^{N} \delta\left(\mathbf{r}-\mathbf{r}_{\mathbf{i}}(t)\right) \delta\left(\mathbf{r}^{\prime}-\mathbf{r}_{\mathbf{j}}(0)\right)\right\rangle .
$$

The spatial Fourier transformation of this equation again leads to the heterodyne autocorrelation function $F_{h e t}(\mathbf{q}, t)$. The space time correlation function only depends on the distance between the particle positions $\mathbf{r}_{\mathbf{i}}-\mathbf{r}_{\mathbf{j}}$ in a uniform system with translational invariance. Thus, without loss of generality the coordinate $\mathbf{r}^{\prime}$ can be set to zero and the transformation between $F_{\text {het }}(\mathbf{q}, t)$ and $\left\langle\rho(\mathbf{r}, t) \rho\left(\mathbf{r}^{\prime}, 0\right)\right\rangle$ can be written as

$$
F_{h e t}(\mathbf{q}, t)=\left\langle\Psi^{*}(t) \Psi(0)\right\rangle=V \int_{V} d^{3} r\langle\rho(\mathbf{r}, t) \rho(0,0)\rangle \exp (i \mathbf{q} \cdot \mathbf{r}),
$$

where $\mathrm{V}$ is the volume of the system.

Here, a function widely used in DLS has to be introduced. The space time correlation function of the particle density distribution in equation (2.35) is proportional to the Van Hove space time correlation function $G(\mathbf{r}, t)$. It was introduced for the first time by Leon Van Hove 
in neutron scattering in 1954 [24].

$$
\langle\rho(\mathbf{r}, t) \rho(0,0)\rangle=\frac{1}{V}\left\langle\sum_{i=1}^{N} \sum_{j=1}^{N} \delta\left(\mathbf{r}-\left[\mathbf{r}_{\mathbf{i}}(t)-\mathbf{r}_{\mathbf{j}}(0)\right]\right)\right\rangle=: \widehat{\rho} G(\mathbf{r}, t),
$$

where $\widehat{\rho}$ is the average particle density of the system and $V$ the system volume. $G(\mathbf{r}, t)$ is a probability distribution function in real space. $G(\mathbf{r}, t) d^{3} r$ can be understood as the probability to find the same or a different particle at the time $t$ in a volume around the origin of $d^{3} r$ when there was a particle at the origin at $t=0$.

The double sum in equation (2.37) can be divided into two parts with indices for same particles $i=j$ and different particles $i \neq j$.

$$
G(\mathbf{r}, t)=G_{S}(\mathbf{r}, t)+G_{D}(\mathbf{r}, t) .
$$

The contribution of the same particles $G_{S}(\mathbf{r}, t)$ is called self Van Hove function which is particularly important for independent particles as will be shown in the following.

\subsubsection{Diffusive and Ballistic Motion of Independent Particles}

In a lot of cases, the positions of the scattering particles are statistically independent, e.g. neutral molecules, atoms or macroscopic particles in solution or systems that act like a perfect gas. Even interacting particles can be treated like independent particles if the characteristic length scale $q^{-1}$ defined by the scattering vector is small compared to the interaction length (see section 2.2.3).

The distances between independent particles are distributed equally, i.e. the positions of different particles are not correlated in time or space. It follows, the second term of the Van Hove function $G_{D}(\mathbf{r}, t)$ is constant and all information about the particle movement can be found in the self Van Hove function $G_{S}(\mathbf{r}, t)$.

The same holds for the heterodyne scattering function $F_{\text {het }}(\mathbf{q}, t)$ given by equation (2.36). The heterodyne scattering function reduce to

$$
F_{h e t}(\mathbf{q}, t)=\left\langle\sum_{i=1}^{N} \exp \left(i \mathbf{q} \cdot\left[\mathbf{r}_{\mathbf{i}}(t)-\mathbf{r}_{\mathbf{i}}(0)\right]\right)\right\rangle .
$$

As long as the particles are statistically independent, the quantity

$$
F_{S}(\mathbf{q}, t):=\left\langle\exp \left(i \mathbf{q} \cdot\left[\mathbf{r}_{\mathbf{i}}(t)-\mathbf{r}_{\mathbf{i}}(0)\right]\right)\right\rangle
$$

is the same for all particles. It represents an ensemble average. This function is also called the self-intermediate scattering function and is the Fourier transform of the self Van Hove 
function

$$
G_{S}(\mathbf{r}, t):=\langle\delta(\mathbf{r}-[\mathbf{r}(t)-\mathbf{r}(0)])\rangle .
$$

The heterodyne scattering function $F_{h e t}(\mathbf{q}, t)$ becomes

$$
F_{\text {het }}(\mathbf{q}, t)=\langle N\rangle F_{S}(\mathbf{q}, t) \text {, }
$$

where $\langle N\rangle$ is the average particle number in the scattering volume. The determination of $F_{S}(\mathbf{q}, t)$ and accordingly $G_{S}(\mathbf{r}, t)$ needs a theoretical description matching the physical properties of the system. The following paragraphs discuss the most important cases for systems of independent particles.

\section{Particle Diffusion}

In the diffusive regime, the particles undergo many collisions on the length scale $q^{-1}$. As mentioned, the self Van Hove function $G_{S}(\mathbf{r}, t) d^{3} r$ can be interpreted as a time space probability function for particle positions. From theory of random walk, it is well known that the diffusion equation describes this probability. That means, the self Van Hove function $G_{S}(\mathbf{r}, t)$ solves the diffusion equation [19]

$$
\frac{\mathrm{d}}{\mathrm{d} t} G_{S}(\mathbf{r}, t)=D \nabla^{2} G_{S}(\mathbf{r}, t),
$$

where $D$ denotes the diffusion constant. The spatial Fourier transform of this equation gives the diffusion equation for the self-intermediate scattering function $F_{S}(\mathbf{q}, t)$

$$
\frac{\mathrm{d}}{\mathrm{d} t} F_{S}(\mathbf{q}, t)=D \nabla^{2} F_{S}(\mathbf{q}, t)
$$

With the boundary condition $F_{S}(\mathbf{q}, 0)=1$, the solution is

$$
F_{S}(\mathbf{q}, t)=\exp \left(-q^{2} D t\right)
$$

The heterodyne scattering function $F_{h e t}(\mathbf{q}, t)$ is therefore a single exponential function with a decay time $\tau_{q}=\left(q^{2} D\right)^{-1}$. The corresponding power spectrum $\widetilde{I}_{h e t}$ is a Lorentzian with half-width at half maximum of $\omega_{h}=q^{2} D$.

$$
\begin{aligned}
F_{h e t}(\mathbf{q}, t) & =\langle N\rangle \exp \left(-q^{2} D t\right) \\
\widetilde{I}_{h e t}\left(\mathbf{q}, \omega_{f}\right) & =\frac{\langle N\rangle}{\pi}\left[\frac{q^{2} D}{\omega_{f}^{2}+\left(q^{2} D\right)^{2}}\right] .
\end{aligned}
$$


The Siegert relation (equation (2.24) ) can be used to obtain the homodyne correlation function

$$
F_{\text {hom }}(\mathbf{q}, t)=\langle N\rangle^{2}\left\{1+\exp \left(-2 q^{2} D t\right)\right\} \equiv\langle N\rangle^{2}\left\{1+\exp \left(-\frac{t}{\tau_{q}}\right)\right\} .
$$

The homodyne correlation function has a baseline of $\langle N\rangle^{2}$ instead of $\langle N\rangle$ and the attained decay time is smaller by a factor of 2 than that measured in heterodyne experiments. Hence, the diffusion constant $D$ in a homodyne experiment can be obtained from the decay time by

$$
D=\frac{1}{2 q^{2} \tau_{q}}
$$

The diffusion constant reveals information about the particle radius via the Einstein relation

$$
D=\frac{k_{B} T_{d}}{\zeta}
$$

where $k_{B}, T_{d}$, and $\zeta$ denote the Boltzmann constant, the dust particle temperature, and the friction constant of the background medium. In the Stokes regime, the friction constant is related to the particle radius by

$$
\zeta=6 \pi \eta a,
$$

where $\eta$ is the viscosity of the medium and $a$ the particle radius.

\section{Ballistic Particle Motion}

In the case of infrequent collisions of the scatterers on the length scale $q^{-1}$, the system acts like a perfect gas (ballistic motion) with freely moving scatterers. The particles move on linear trajectories along $q^{-1}$ with a velocity

$$
\mathbf{v}_{\mathbf{j}}=\frac{\mathbf{r}_{\mathbf{j}}(t)-\mathbf{r}_{\mathbf{j}}(0)}{t}
$$

Thus, the self-intermediate scattering function can be written as

$$
F_{S}(\mathbf{q}, t)=\left\langle\exp \left(i \mathbf{q} \cdot \mathbf{v}_{\mathbf{j}} t\right)\right\rangle
$$

This is the average of the expression $\exp \left(i \mathbf{q} \cdot \mathbf{v}_{\mathbf{j}} t\right)$ over the velocity distribution $P(\mathbf{v})$. If a Maxwell distribution is assumed, the average becomes

$$
F_{S}(\mathbf{q}, t)=\left[\frac{m_{p}}{2 \pi k_{B} T_{D}}\right]^{\frac{3}{2}} \int d^{3} v \exp \left(-\frac{m_{p} v^{2}}{2 k_{B} T_{D}}\right) \exp \left(i \mathbf{q} \cdot \mathbf{v}_{\mathbf{j}} t\right)
$$

where $m_{p}$ the mass of the dust particles. If we choose $\mathbf{q}$ along the $\mathbf{x}$-axis $\left(\mathbf{q} \cdot \mathbf{v}=q v_{x}\right)$ the 
integral for $v_{y}$ and $v_{z}$ can be solved and the final integral over $v_{x}$ yields [13, p.66]

$$
F_{S}(\mathbf{q}, t)=\exp \left(-\frac{1}{2} q^{2}\left\langle v_{x}^{2}\right\rangle t^{2}\right)
$$

where $\left\langle v_{x}^{2}\right\rangle$ gives the average squared velocity component in x-direction. The heterodyne scattering function becomes

$$
F_{h e t}(\mathbf{q}, t)=\langle N\rangle \exp \left(-\frac{1}{2} q^{2}\left\langle v_{x}^{2}\right\rangle t^{2}\right)
$$

and by applying the Siegert relation, the correlation function of a homodyne experiment is

$$
F_{\text {hom }}(\mathbf{q}, t)=\langle N\rangle^{2}\left\{1+\exp \left(-\frac{1}{2} q^{2}\left\langle v_{x}^{2}\right\rangle t^{2}\right)^{2}\right\} \equiv\langle N\rangle^{2}\left\{1+\exp \left(-\frac{2}{\omega_{q}^{2}} t^{2}\right)\right\},
$$

where $\omega_{q}$ is the full width of a Gaussian function.

The one-dimensional average velocity-squared can be obtained from the width of the Gaus$\operatorname{sian} \omega_{q}$ via

$$
\left\langle v_{x}^{2}\right\rangle=\frac{2}{\omega_{q}^{2} q^{2}}=\frac{1}{2 \sigma^{2} q^{2}}
$$

where in the last expression $2 \sigma=\omega_{q}$ is used. Assuming an isotropic two-dimensional system, the average velocity-squared is expressed by

$$
\left\langle v_{2 D}^{2}\right\rangle=\frac{4}{\omega_{q}^{2} q^{2}}=\frac{1}{\sigma^{2} q^{2}}
$$

using $\left\langle v_{2 D}^{2}\right\rangle=\frac{1}{2}\left\langle\left(v_{x}^{2}+v_{y}^{2}\right)\right\rangle$. If the equation (2.56) is plotted against $q t$, it will be independent of the scattering angle. The theoretical form for diffusive motion (equation 2.47), however, will be independent of the scattering angle if it is plotted against $q^{2} t$. This can be used as a test to distinguish between free motion and diffusive motion. 



\section{Chapter 3}

\section{Complex Plasmas}

Complex or dusty plasmas consist of electrons, ions, neutrals, and macroscopic solid dust particles of nano or micron size. The dust grains attain a charge that can be positive or negative depending on the charging mechanism. In most laboratory experiments, the dust is mainly charged by the inflow of electrons and ions, and acquires a negative charge. The grain charge typically reaches the order of $10^{3}-10^{5}$ elementary charges and for this reason the dust particle component is coupled much stronger than other plasma components. This makes complex plasmas a unique subject to study strong interactions on fast time scales. The charging mechanisms of dust grains immersed in a plasma are discussed in section 3.1

The inter-particle interaction of the dust grains is determined by the dust grain potential $\phi(r)$. The potential $\phi(r)$ can be described by a screened Coulomb potential, the Debye-Hückel (Yukawa) potential

$$
\phi(r)=\phi_{s} \frac{a}{r} \exp \left(\frac{r-a}{\lambda_{D}}\right),
$$

where $\phi_{s}$ denotes the potential at the surface of the grain, $a$ is the radius of the dust grain and $\lambda_{D}$ denotes the screening length scale. This is given by the total Debye length, that is defined by $\lambda_{D}^{-2}=\lambda_{D e}^{-2}+\lambda_{D i}^{-2}$, where $\lambda_{D e(i)}=\sqrt{\epsilon_{0} k_{B} T_{e(i)} /\left(e^{2} n_{0}\right)}$ is the electron (ion) Debye length. $T_{e(i)}$ is the electron (ion) temperature and $n_{0}$ is the plasma density. The Debye length defines the length scale on which the grain potential is shielded by the ambient plasma. The derivation of the Debye-Hückel potential and a discussion of the validity of this potential are presented in section 3.2.1

Micron-scaled dust grains can be confined in a radio frequency discharge, as done in the experiments presented in this work. External forces that act on the dust grains, like gravity and ion drag force, are compensated by the electric force in the plasma-wall sheath of the lower electrode. Section 3.2 is dedicated to all the forces acting on the dust grains in a plasma.

The thermal motion of the dust component can be considerably reduced by friction with the background gas. If the electrostatic interaction energy of the dust grains exceeds their thermal energy, a strongly coupled stated is reached. The dust grains arrange to well ordered lattice structures, sometimes called plasma crystals. Two-dimensional as well as three-dimensional crystal structure can be realized. Two-dimensional crystals are basically a single layer of ordered dust grains, whereas three-dimensional crystals can consist of hundreds of layers. 
The reduction of the gas friction by lowering the discharge pressure induces a phase transition into a disordered phase state. The particles attain unexpected large thermal energies in the melted state. The structures and phase transitions of complex plasmas are treated in section 3.3 .

\subsection{Charging of Dust Grains in a Plasma}

The electrostatic charge of a dust grain is the most fundamental parameter, that determines the structure and dynamics of the dust ensemble. It governs the interaction between the dust grains and is the reason for the strong coupling of the dust component. A proper description of the charging process is the first step for any model on complex plasmas.

A fundamental approach, although based on strongly idealized assumptions, is the Orbital Motion Limited (OML) theory introduced by Mott-Smith and Langmuir [25, 26, 27]. Here, the thermal fluxes of plasma particles onto the grain surface are considered to determine the dust grain charge. The basics of the OML approach are discussed in section 3.1.1 and an approximative expression for the grain charge is presented.

The assumptions of the OML approach are not always valid in complex plasmas. In section 3.1.2, the OML approach is extended to consider the conditions in plasma experiments more precisely.

There are other mechanisms beside the charging by thermal fluxes of plasma particles that affect the grain charge. Section 3.1.3 gives a short overview over further charging mechanisms in dusty plasmas.

A variation of the grain charge has serious consequences on the dynamical processes taking place. In section 3.1.4 the impact of charge fluctuations on the dust ensemble are discussed.

\subsubsection{OML Theory}

If electron emission processes from the grain surface are negligible, thermal fluxes of electrons and ions onto the grain surface are responsible for the grain charging. Because of the higher thermal velocity of the electrons, the dust grain gets negatively charged. The increasing negative dust charge more and more repels the electrons and attracts the positive ions. Finally, the balance of the electron and ion fluxes determine the magnitude of the grain charge [27].

The electron and ion fluxes are predicted by probe theory. In dusty plasmas, the Orbital Motion Limited (OML) approach is most commonly used. The grain charge is determined by the orbital motion of electrons and ions in the grain potential. The fluxes to the grain surface can be derived by conservation laws of energy and angular momentum. This, in general, does not depend on the specific form of the interaction potential. But, to assure orbital motion of electrons and ions, some assumptions have to be made in the OML approach [28, 29, 30]:

- There are no barriers in the effective potential of the dust grain. 
- Electrons and ions do not experience collisions on their trajectories toward the dust grain.

- The dust grain is isolated, i.e. no other grains disturb the orbital motion of electrons and ions.

- The plasma is isotropic with Maxwell-Boltzmann velocity distributions of electrons and ions.

The electron (ion) current to the particle surface is obtained by the integral over the velocity distribution $f_{e(i)}(v)$ and the cross section for collection of electrons (ions) $\sigma_{e(i)}(v)$

$$
I=e n_{e(i)} \int v \sigma_{e(i)}(v) f_{e(i)}(v) d^{3} v,
$$

where $n_{e(i)}$ gives the electron (ion) number density. The collection cross sections are derived by the OML approach.

An ion that approaches the dust grain from infinity has an energy balance that can be written as

$$
\frac{1}{2} m_{i} v_{i, 0}^{2}=\frac{1}{2} m_{i} v_{i}^{2}+e \phi_{s}
$$

where $m_{i}$ is the ion mass, $v_{i, 0}$ gives the velocity at infinity and $\phi_{s}$ is the surface potential of the dust grain. The conservation of angular momentum is used now to derive an expression for the maximal impact parameter for a collision on the grain surface $\rho_{c}$ [29]. The collection cross section is given by $\sigma_{i}=\pi \rho_{c}^{2}$, that finally leads to

$$
\sigma_{i}=\pi a^{2}\left(1-\frac{2 e \phi_{s}}{m_{i} v_{i, 0}^{2}}\right),
$$

where $a$ is the dust grain radius. Here, the grain surface potential is negative $\left(\phi_{s}<0\right)$ and the ions are singly charged.

A similar derivation leads to the collision cross section for electrons

$$
\sigma_{e}=\pi a^{2}\left(1+\frac{2 e \phi_{s}}{m_{e} v_{e, 0}^{2}}\right),
$$

where $m_{e}$ is the electron mass. This cross section is valid for the case $e \phi_{s}<\frac{1}{2} m_{e} v_{e, 0}^{2}$. In the opposite case $\left(e \phi_{s} \geq \frac{1}{2} m_{e} v_{e, 0}^{2}\right)$ the collection cross section for electrons is zero $\sigma_{e}=0$. The combination of the collection cross sections (equations (3.5) and (3.4) with equation (3.2), assuming Maxwell-Boltzmann velocity distributions for both species, finally gives the ion and electron currents to the grain surface

$$
I_{i}=\sqrt{8 \pi} a^{2} e n_{i} v_{T_{i}}\left(1-\frac{e \phi_{s}}{k_{B} T_{i}}\right)
$$




$$
I_{e}=-\sqrt{8 \pi} a^{2} e n_{e} v_{T_{e}} \exp \left(\frac{e \phi_{s}}{k_{B} T_{e}}\right)
$$

where $v_{T_{e(i)}}=\sqrt{k_{B} T_{e(i)} / m_{e(i)}}$ is the thermal velocity of the electrons (ions).

The potential of the dust grain (floating potential) is determined by the balance of the particle currents $I_{i}=-I_{e}$ :

$$
1-\frac{e \phi_{s}}{k_{B} T_{i}}=\sqrt{\frac{m_{i} T_{e}}{m_{e} T_{i}}} \frac{n_{e}}{n_{i}} \exp \left(\frac{e \phi_{s}}{k_{B} T_{e}}\right) .
$$

The surface potential $\phi_{s}$ can be obtained by solving this equation numerically. As shown by Goree et al. [27], the surface potential shows to be proportional to the electron temperature $\phi_{s} \propto T_{e}$.

The grain charge can be approximated by regarding the dust particle as a spherical capacitor [28]. The dust grain charge can be written as $Q_{d}=Z_{d} e=C_{d} \phi_{s}$, where $Z_{d}$ is the number of charges on the dust particle and $C_{d}$ denotes the capacitance. The capacitance of a dust grain in a plasma is given by $C_{d}=4 \pi \epsilon_{0} a\left(1+a / \lambda_{D}\right)$, where $\lambda_{D}$ is the Debye length and $a$ is the dust grain radius [31]. For the case $\lambda_{D} \gg a$, which is satisfied for typical experimental conditions, the capacitance in vacuo can be used and the equation of $Q_{d}$ simplifies to

$$
Q_{d}=4 \pi \epsilon_{0} a \phi_{s}
$$

Numerical analyses of equation (3.8) are performed by Goree et al. in [27]. The charge number obtained there (by using equation (3.9) ) is of the order of $Z_{d}=8 \cdot 10^{4}$ for particles with radius of $10 \mu \mathrm{m}$. The calculations are done assuming an argon plasma with an electron temperature of $k_{B} T_{e}=4 \mathrm{eV}$ and $T_{e} / T_{i}=20$.

The experimental determination of the dust grain charge in the plasma-wall sheath of an argon RF-plasma results in charge numbers between $1.5 \cdot 10^{5}$ and $2.5 \cdot 10^{5}$ [32].

The discrepancy between the numerical values and the results of experiments has its origin in the violation of the assumptions made in the OML approach. Some extensions of the OML theory, to describe the experimental conditions more precisely, are discussed in the following section.

\subsubsection{Extensions of the OML Approach}

\section{Barrier Effects}

The radial motion of an ion with velocity $v$ in the vicinity of a charged dust grain is determined by the screened potential of the grain and the angular momentum of the ion. For a certain type of grain potential $\phi(r)$, the effective potential shows barriers. These barriers can reflect ions that would have been collected in the OML approach, where potential barriers are 
neglected. This reduces the ion current and therefore, the dust gets charged more negative.

To determine the properties of the grain potential $\phi(r)$, that induce potential barriers in the effective potential, an ion in the attractive central field of a dust grain is considered. The energy of the ion is written as

$$
E=\frac{1}{2} m_{i}\left(v_{r}^{2}+v_{\theta}^{2}\right)+e \phi(r)
$$

where $v_{r}$ is the radial velocity and $v_{\theta}$ is the tangential velocity. The movement of the ion in the central field can be interpreted as a one dimensional (radial) motion in an effective potential $U_{e f f}(r)$. Using the angular momentum $L=m_{i} r v_{\theta}$, the equation above becomes

$$
E=\frac{1}{2} m_{i} v_{r}^{2}+\underbrace{\frac{L^{2}}{2 m_{i} r^{2}}+e \phi(r)}_{:=U_{e f f}(r)} .
$$

The last two terms of the sum on the right hand side define the effective potential $U_{e f f}(r)$.

A discussion of the extrema of $U_{e f f}(r)$ gives further insight into the shape of the effective potential [33]:

$$
\frac{d U_{e f f}(r)}{d r}=0 \quad \Leftrightarrow \quad r^{3} \frac{d(e \phi(r))}{d r}=\frac{L^{2}}{m_{i}}
$$

The right hand equation shows that the shape of $U_{e f f}(r)$ is determined by the behavior of the grain potential $\phi(r)$ for a given angular momentum $L$. It turns out, if $\phi(r)$ decreases everywhere more slowly than $r^{-2}$, equation (3.12) only has one solution and the effective potential shows a single minimum. If $\phi(r)$ decreases slower than $r^{-2}$ at small $r$, and decreases faster than $r^{-2}$ at larger $r$, the effective potential has a minimum and a maximum. In this case, a barrier in $U_{\text {eff }}(r)$ appears [33, 29, 30].

In complex plasmas, the grain potential can be described by a Debye-Hückel (Yukawa) potential $\phi(r)=-\left(\phi_{0} / r\right) \exp \left(-r / \lambda_{D}\right)$. Under certain conditions, this potential can be of the second type, where potential barriers appear. In this case, reflections of a part of the ions on potential barriers has to be considered.

The presence of barriers in $U_{e f f}(r)$ for a Debye-Hückel grain potential was demonstrated by Fortov et al. in [29]. Figure 3.1] shows the effective potential for different values of $\rho / \lambda_{D}$, the impact parameter normalized to the Debye length 2 . The values of the parameter $\rho / \lambda_{D}$ are indicated in the figures. The curves in (a) and (b) are calculated for different scattering parameters $\beta$ (Fortov et al. [29]). This dimensionless parameter is defined as $\beta=\left|e \phi_{0}\right| /\left(4 \pi \epsilon_{0} m v_{0}^{2} \lambda_{D}\right)$ and describes the "strength" of interactions via the Debye-Hückel potential. In figure (a) at $\beta=10$, the potential decreases everywhere faster than $r^{-2}$ and no barrier is observed. In figure (b) at $\beta=20$, barriers are present for different angular momenta.

Khrapak et al. discuss the applicability of the OML approach according to the presence of

${ }^{2}$ In the figure, the screening length is denoted by $\lambda$ instead of $\lambda_{D}$. 
(a)

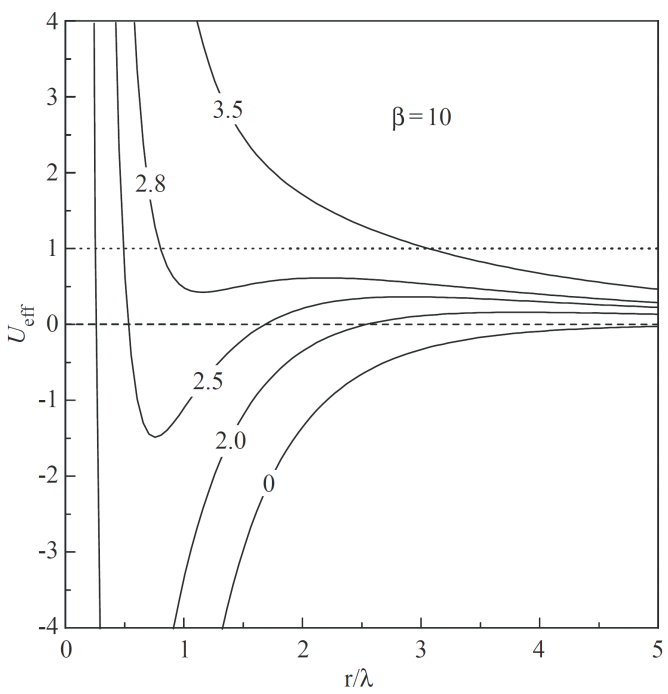

(b)

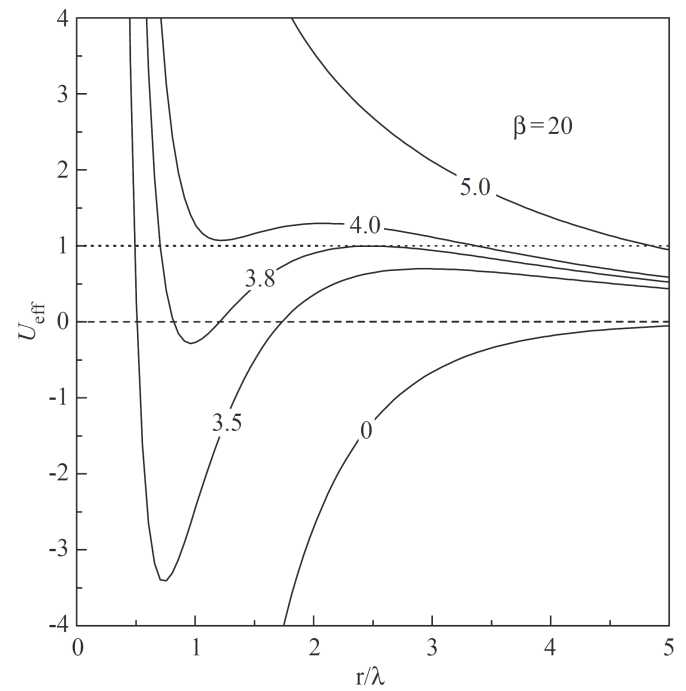

Figure 3.1: Curves of the effective potential $U_{e f f}(r)$ for the radial ion motion in the central field of a Debye-Hückel potential. Two different scattering parameters $\beta$ and different normalized impact parameter $\rho / \lambda_{D}\left(\lambda_{D}\right.$ is denoted as $\lambda$ in the figure) are shown. In (a) the potential barriers are absent for $\beta=10$, whereas in (b) barriers in the effective potential can be seen for $\beta=20$. (The figures are taken from Fortov et al. [29].)

potential barriers. They give a condition that can be written in the form

$$
\sqrt{2 z} \frac{T_{e}}{T_{i}} \frac{a}{\lambda_{D}} \leq \ln \left(z \frac{T_{e}}{T_{i}} \frac{a}{\lambda_{D}}\right)
$$

where the quantity $z=Z_{d} e^{2} /\left(4 \pi \epsilon_{0} a k_{B} T_{e}\right)$ is the dimensionless charge [34]. For typical complex plasma parameters $\left(z \approx 1\right.$ and $\left.T_{e} / T_{i} \approx 100\right)$, the condition simplifies to $a / \lambda_{D} \leq 0.2$.

The barriers in the effective potential are absent for repulsive interactions. Thus, the charging currents of the electrons are not affected for the case of a negatively charged grain.

\section{Ion-neutral Collisions}

An important deviation from the OML approach is caused by ion-neutral collisions. The omission of collisions in the OML theory was justified by the condition, that the ion mean free path $l_{i}$ is long compared to the screening length $\lambda_{D}$ [27]. Extended theories show, that ionneutral collisions can significantly influence the charging process, even in the case of $l_{i} \gg \lambda_{D}$.

When an incoming ion loses energy in a collision with a cold atom near the dust grain, the ion cannot escape the grain attraction and is collected on the surface, if the angular momentum after the collision is sufficiently low. If the ion has a larger angular momentum, it will probably orbit around the grain and be trapped in the potential well of the dust grain [35, 36]. The trapped ions are likely to fall onto the grain surface after further collisions and therefore 
increase the ion current to the surface too.

The ion-neutral charge-exchange collisions are in particular effective in increasing the ion current. In such a collision, an accelerated ion and a cold neutral atom are replaced by a cold ion and a fast neutral. Due to the low ion energy after the collision, nearly every ion produced by such a collision is collected on the grain surface.

Lampe et al. [36] derived an expression for the ion current including the effects of ionneutral charge-exchange collisions and trapped ions. Following this work, the introduction of the radius $R_{T}$ is useful, that is defined by $\phi\left(R_{T}\right)=-3 / 2 k_{B} T_{i}$. At the distance $R_{T}$ from the grain center, the potential energy is equal to the average energy of an ion after a chargeexchange collision. Thus, if an ion undergoes a collision within the sphere with radius $R_{T}$, it cannot escape the grain potential. Here, only the weakly collisional regime is discussed, where $l_{i} \gtrsim R_{T}$.

The collection area of ions is now $\pi R_{T}^{2}$, which is much larger than the particles cross section. The corresponding particle current is $I_{R_{T}}=\sqrt{8 \pi} R_{T}^{2} n_{i} v_{T_{i}}$, where $v_{T_{i}}$ is the thermal velocity of the ions with temperature $T_{i}$. Because not every ion that passes the sphere with radius $R_{T}$ is collected, the current $I_{R_{T}}$ has to be weighted by the probability of charge-exchange collisions. This probability is of order $R_{T} / l_{i}$. The current of ions due to charge-exchange collision can therefore be written as $I_{\text {coll }}=\left(R_{T} / l_{i}\right) I_{R_{T}}$. In combination with the OML current $I_{i}$ (equation (3.6)), an approximate expression for the collisional ion current is obtained $I_{i, \text { coll }}=\left(1-\left(R_{T} / l_{i}\right)\right) I_{i}+\left(R_{T} / l_{i}\right) I_{R_{T}}$, i.e.

$$
I_{i, \text { coll }}=\sqrt{8 \pi} a^{2} e n_{i} v_{T_{i}}\left(1-\frac{e \phi_{s}}{k_{B} T_{i}}+\frac{R_{T}^{3}}{a^{2} l_{i}}\right)
$$

where $a$ is the grain radius, $n_{i}$ the ion number density, and $k_{B} T_{i}$ is the thermal energy of the ions in the ambient plasma.

This expression for the collection current is an underestimate, because ion-neutral collisions outside the sphere with radius $R_{T}$ contribute to the ion current too, although with a lower probability of collection.

Fortov et al. [29] pointed out, that collisions can affect particle charging even if the mean free path of the ions $l_{i}$ is an order of magnitude larger than the screening length $\lambda_{D}$. This makes the consideration of collisions a major correction of the OML approach.

\section{Electron Depletion}

The OML approach describes the ideal case of an isolated dust grain or probe immersed in a plasma. In a complex plasma experiment however, the number of dust particles is huge and the distance between neighboring dust particles can become of the order or even smaller than the Debye length $\lambda_{D}$.

The assumption of undisturbed particle orbits is not satisfied in this case. The trajectories of ions and electrons are influenced by interactions with close neighboring particles and this 
affects the ion and electron currents onto the grain surface.

Furthermore, the presence of many dust grains affects the number density of unbounded electrons. The condition of quasi-neutrality for a dusty plasma with singly charged ions is written as

$$
n_{e}=n_{i}-Z_{d} n_{d}
$$

where $n_{e}, n_{i}$, and $n_{d}$ are the number densities of electrons, ions, and dust grains, and $Z_{d}$ is the number of charges on the dust grains. The lower number density of unbounded electrons reduces the local plasma potential compared to the dust free plasma. The potential difference between the local plasma and the grain surface is lower, thus the grain potential is more positive [37.

Havnes et al. have demonstrated, that the potential difference and the resulting dust charge only depends on the Havnes parameter $P_{H}=n_{d} Z_{d} / n_{e}$ [38, 27]. The charge on the grains is close to the value of isolated grains for $P_{H} \ll 1$, and it is strongly reduced in the opposite case $P_{H} \gg 1$. The predicted reduction of the grain charge due to electron depletion was demonstrated experimentally for example by Barkan et al. [39] and Xu et al. [40].

\section{Streaming Ions}

The assumption of an isotropic plasma environment with Maxwell-Boltzmann velocity distributions of electrons and ions is most often violated in complex plasma experiments. The presence of electric fields accelerates charged plasma particles and leads to a streaming plasma environment.

Examples for streaming plasma conditions are the electric field in the striations of a DCdischarge or the outward directed ion streams responsible for void formation in dust clouds of nano-scaled dust particles [41]. In this work, dust grains of micron size are confined near the lower electrode of a RF-discharge. Here, the electric field of the plasma-wall sheath is used to balance the gravity force and thus levitate the dust grains. The ions travel at sonic or supersonic velocities (Bohm-criterion) and the sheath is apparently an anisotropic plasma environment. The streaming plasma particles affect the charging process and potential distribution of the dust grain.

In the plasma-wall sheath, the impact of the anisotropy on the grain charge also depends on the position of the dust grain. The ions enter the sheath with Bohm-velocity $v_{B}=\sqrt{k_{B} T_{e} / m_{i}}$ and are further accelerated in the parabolic sheath potential [42]. Hence, the ion streaming velocity $u_{i}$ is larger close to the electrode as compared to the presheath and the bulk of the plasma. If the dust grain is located in the pre-sheath, the ion streaming velocity is subsonic $\left(u_{i}<v_{T_{i}}\right)$ and the grain charge can be described by the OML approach [29].

Only the streaming ions have to be considered in the derivation of the grain charge. The electron streaming can be neglected, because the streaming speed is smaller than the thermal velocity of the electrons. Thus, the electrons still appear to be isotropic, and the OML 
Figure 3.2: Dimensionless charge $z=Z_{d} e^{2} /\left(4 \pi \epsilon_{0} a k_{B} T_{e}\right)$ versus the ratio of streaming speed and thermal velocity of the ions $u_{i} / v_{T_{i}}$ (lower $\mathrm{x}$-axis) and the Mach-number $M=u_{i} / C_{I A}$ (upper $\mathrm{x}$-axis). Three different ratios of $n_{e} / n_{i}$ are shown for an argon plasma with $T_{e} / T_{i}=100$. At low $u_{i} / v_{T_{i}}$, the situation corresponds to the OML conditions. For higher $u_{i} / v_{T_{i}}$, the charge increases, reaches a maximum, and starts to decrease. The decrease is caused by a positive space charge $\left(n_{i}>n_{e}\right)$ that develops at this conditions. (The graph is taken from Fortov et al. [29].)

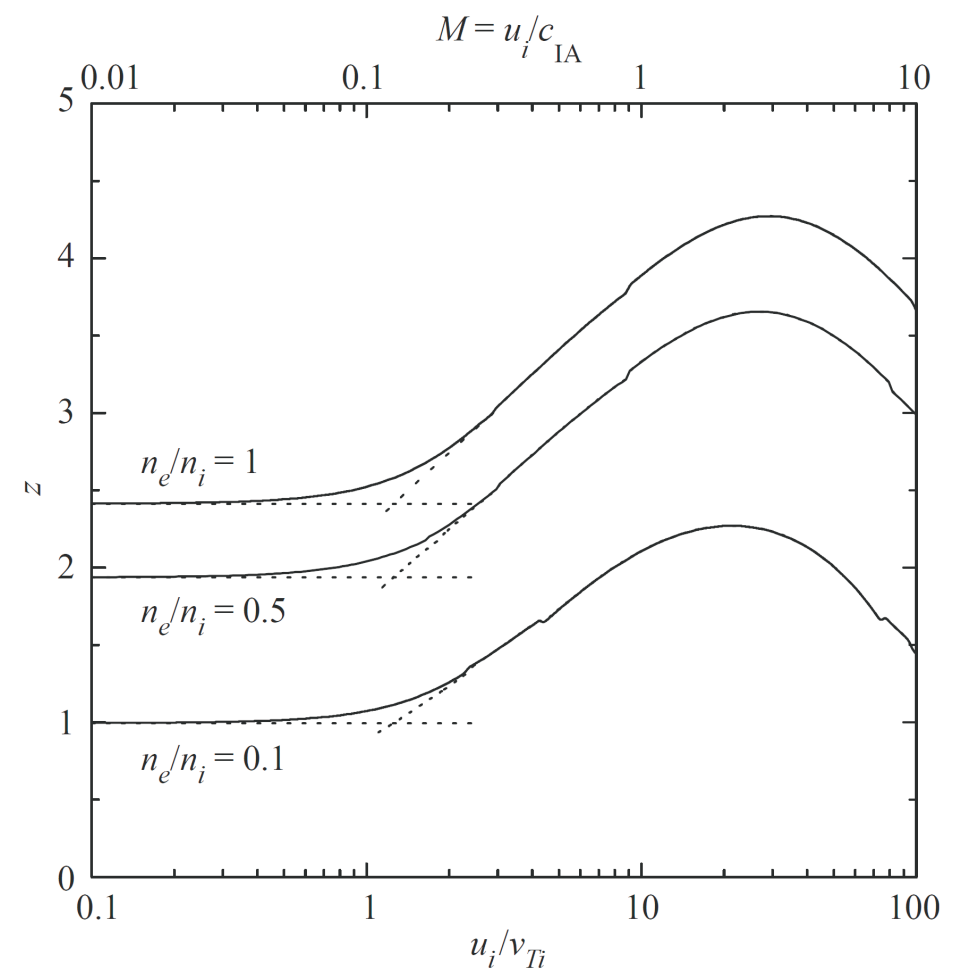

approach is valid to describe the electron currents $I_{e}$ (equation (3.7)).

The anisotropy of the plasma changes the collection cross section and velocity distribution of the ions. The exact treatment of the charging process is rather complex, but the ion current can also be estimated by a small adaption of the OML approach. In this adaption, a shifted Maxwell-Boltzmann velocity distribution is assumed for the ions to derive the particle current onto the grain surface. These calculations are performed for example by Whipple et al. in [31] and by Uglov et al. in [43]. The result can be simplified under the assumption $u_{i} \gg v_{T_{i}}$. In this case, the ion collection current can be written as

$$
I_{i}=\pi a^{2} n_{i} e u_{i}\left(1-\frac{2 e \phi_{s}}{m_{i} u_{i}^{2}}\right)
$$

where $a$ is the grain radius, $n_{i}$ and $m_{i}$ are the number density and mass of the ions, and $\phi_{s}$ is the grain surface potential.

The balance of the anisotropic ion current (equation (3.16) with the OML electron current (equation (3.7) ) can be used to determine the grain charge numerically as a function of the ion streaming velocity $u_{i}$.

Fortov et al. [29] calculated the grain charge in the plasma-wall sheath, where the ion streaming speed increases toward the wall. The calculations are done for different ratios of $n_{e} / n_{i}$, as shown in figure 3.2. The figure depicts the dimensionless charge $z$ versus the ratio 
of streaming speed and thermal velocity of the ions $u_{i} / v_{T_{i}}$ (lower x-axis) and the Mach-number $M=u_{i} / C_{I A}$ (upper x-axis). The expression $C_{I A}=\sqrt{k_{B} T_{e} / m_{i}}$ is the ion-acoustic phase velocity (ion sound speed).

The figure 3.2 shows that the dimensionless charge $z=Z_{d} e^{2} /\left(4 \pi \epsilon_{0} a k_{B} T_{e}\right)$ corresponds to the OML value at low ion streaming velocities $u_{i}$. When the streaming velocity gets larger than the thermal velocity of the ions, the charge of the dust grain starts to rise. A decrease of the charge $z$ is observed for a streaming velocity $u_{i}$ that is several times larger than the thermal velocity. Here, the situation corresponds to the region near the wall, where the electron density is lower than the ion density $n_{i}>n_{e}$ and a positive space charge develops. This is the reason for the observed decrease of the grain charge.

The presented extension of the OML theory by taking into account drifting ions appears to be accurate for electrically conducting dust grains [44, 45]. This is not true for dust grains of dielectric material. Self-consistent kinetic simulations using the Particle-In-Cell-method reveal a polarization of the charge distribution on the grain surface for dielectric dust grains [46]. The lower ion flux on the downstream side results in a more negative potential. This substantially increases the grain charge for supersonic ion flows [44, 45].

\subsubsection{Other Charging Effects}

The charging currents due to thermal fluxes of plasma particles are not the only possible charging mechanisms. Under certain experimental conditions, the charging by photoelectric emission, i.e. the emission of photo electrons after absorption of UV radiation, can be important. The impact of UV radiation on the grain charge was studied experimentally with an external UV source in [47, 48]. The thermal emission of electrons can be of importance in thermal dusty plasmas as for example in combustion flames [49]. The secondary emission of electrons by the impact of electrons or ions on the grain might also be important, if plasma particles with sufficiently high energies are present.

These electron emission processes require a low work function of the grain material to become important. Thus, for example metal and oxide grains with work functions below $5 \mathrm{eV}$ are prone to these emission processes [50].

The release of electrons can result in a positive grain charge in contradiction to the negative charging discussed so far. This scenario is in particular relevant for nano-scaled dust particles.

The effect of photo electron emission and secondary emission are probably the most important additional charging mechanisms in addition to the thermal fluxes of electrons and ions onto the grain surface [27].

Other processes that can affect the grain charge are field emission, sputtering, and nuclear decay of radioactive dust grains. 


\subsubsection{Temporal Behavior of the Dust Grain Charge}

In the previous discussion on particle charging, it was assumed that the continuous charging currents cause a constant grain charge. In a real complex plasma experiment, the equilibrium grain charge varies due to local fluctuations of the plasma conditions. To estimate the impact on the dust dynamics, the time scale of the charging process is compared to the relevant time scales of dust grain movement. Furthermore, the grain charge fluctuates statistically due to the discrete nature of the charge carriers and their random arrival times [51]. These stochastic fluctuations are found to contribute to the heating of the dust component.

The evolution of the charge is characterized by the charging rate $\Omega_{c h}$, which is the inverse charging time. The charging rate can be calculated by $\Omega_{c h}=d\left(I_{i}-I_{e}\right) /\left.d Q\right|_{Q_{0}}$, where $I_{i}$ and $I_{e}$ are the OML currents for the ions (equation (3.6) ) and the electrons (equation (3.7) 3 . Assuming that $T_{e} / T_{i} \gg 1$, the charging rate can be written as [29, 30]

$$
\Omega_{c h}=\frac{1+z}{\sqrt{2 \pi}} \frac{a}{\lambda_{D i}} \omega_{p i} \propto a n_{i}
$$

where $z=|Z| e^{2} /\left(4 \pi \epsilon_{0} a k_{B} T_{e}\right)$ is the dimensionless charge, $\lambda_{D i}=\left(\epsilon_{0} k_{B} T_{i} / e n_{e}\right)^{1 / 2}$ is the ion Debye length, and $\omega_{p i}=\left(e^{2} n_{e} / \epsilon_{0} m_{i}\right)^{1 / 2}$ is the ion plasma frequency. The equation (3.17) shows, that the fastest charging is obtained for large dust grains and high plasma densities [27].

The charging time is of the order of a microsecond for conditions of a typical complex plasma experiment. This is much faster than the times scales of macroscopic particle movement, and therefore the particle charge can be assumed to adjust to new plasma conditions instantaneously.

The random impact times of electrons and ions result in a statistical fluctuation of the grain charge [51]. This leads to fluctuations of the inter-grain potential and the external forces that act on the dust grain. Vaulina et al. [52, 53] show analytically and by the aid of simulations, that the stochastic charge fluctuations are a reason for the heating of the dust particles. The role of this effect is found to be more important for bigger particles. The thermal energy of the dust particles with charge fluctuations is found to be about $0.2 \mathrm{eV}$ for particles with a radius of $5 \mu \mathrm{m}$ and about $5 \mathrm{eV}$ for $25 \mu \mathrm{m}$ particles, that is considerably higher than the room temperature of the background gas.

\subsection{Forces Acting on a Dust Particle}

Dust grains in a plasma are subject to several forces that determine distribution, dust structure formation, and transport of the particles.

The force between the dust particles is dominated by the electrostatic interaction of the

${ }^{3}$ Other possible charge currents are neglected here. 
dust grain potentials. The form and strength of the potential determine the coupling between the particles, and therefore they are responsible for the dust structures observed in a complex plasma. Section 3.2.1 addresses the grain potential $\phi(r)$ of dust grains in a plasma.

In section 3.2.2, the ion drag force is discussed. This force is due to momentum transfer by collisions of plasma particles with the dust grain. The ion drag introduces an anisotropy into the inter-grain interaction and is essential for the dust structures in complex plasmas.

The force due to external electric fields can be used in ground-based experiments to balance the gravity force. Furthermore, temperature gradients, neutral drag, laser illumination and shadowing effects can exert a force on the dust grains as discussed in section 3.2.3.

\subsubsection{Electrostatic Interaction between Dust Particles}

\section{Dust Grain Potential in an Isotropic Plasma}

The electrostatic potential around the dust grain $\phi(r)$ solves Poisson's equation [29, 10]

$$
\Delta \phi=\frac{e}{\epsilon_{0}}\left(n_{e}(r)-Z_{i} n_{i}(r)\right),
$$

where $n_{e}$ and $n_{i}$ are the number density of electrons and ions and $Z_{i}$ is the charge state of the ions. In low-temperature plasma experiments, the ions are usually singly charged $Z_{i}=1$.

The radial number densities of electrons and ions are assumed to be Boltzmann-distributed

$$
n_{e(i)}(r)=n_{0} \exp \left( \pm \frac{e \phi(r)}{k_{B} T_{e(i)}}\right),
$$

where the plus sign refers to the electrons and the minus sign to the ions. The assumption of Boltzmann distributions for electrons and ions is not always justified, in particular for large grain charges it becomes questionable in the vicinity of the grain.

Replacing the densities in Poisson's equation with equation (3.19) yields the Poisson-Boltzmann equation that describes nonlinear screening of the dust grain potential. In the case $e \phi \ll k_{B} T_{e(i)}$, the exponential can be expanded and the Poisson-Boltzmann equation writes [54]

$$
\Delta \phi=\frac{\phi}{\lambda_{D}^{2}}
$$

where $\lambda_{D}$ denotes the total plasma Debye length $\left(\lambda_{D}^{-2}=\lambda_{D e}^{-2}+\lambda_{D i}^{-2}\right)$, where $\lambda_{D e(i)}$ is the electron (ion) Debye length 4 . In the case of $T_{e} / T_{i} \gg 1$, the screening length is dominated by the ion Debye length, i.e. $\lambda_{D} \approx \lambda_{D i}$.

The solution of the linearized Poisson-Boltzmann equation is the Debye-Hückel (Yukawa)

$$
{ }^{4} \lambda_{D e(i)}=\sqrt{\epsilon_{0} k_{B} T_{e(i)} /\left(e^{2} n_{0}\right)}
$$


potential

$$
\phi(r)=\phi_{s} \frac{a}{r} \exp \left(\frac{r-a}{\lambda_{D}}\right),
$$

where $\phi_{s}$ denotes the surface potential and $a$ is the radius of the dust grain. Using equation (3.9), the surface potential can be written as $\phi_{s}=Z_{d} e /\left(4 \pi \epsilon_{0} a\right)$, under the assumption of small dust particles $a \ll \lambda_{D}$. By replacing $\phi_{s}$, the equation (3.21) simplifies to

$$
\phi(r)=\frac{e Z_{d}}{4 \pi \epsilon_{0} r} \exp \left(-\frac{r}{\lambda_{D}}\right) \text {. }
$$

The Debye-Hückel potential is a useful approximation of the grain potential, albeit no physical justification for this approximation has been given until now [30]. Indeed, several assumption made in the derivation are usually violated in complex plasmas:

The condition $e \phi \ll k_{B} T_{e(i)}$ does not hold for the ions in a typical dusty plasma experiment. Thus, the linearization of the ion Boltzmann distribution is questionable. Furthermore, a Boltzmann distribution does not describe the distribution of electrons and ions in the vicinity of a charged dust grain very accurate. This is in particular true for attracted particles, e.g. ions in a negative grain potential. Finally, the absorption of plasma particles on the grain surface makes the distribution functions anisotropic in the velocity space [29].

A more realistic grain potential $\phi(r)$ is described in numerical calculations by Kennedy and Allen [55]. This dust grain potential scales like $r^{-1}$ close to the particle surface and like $r^{-2}$ far from the grain. At intermediate distances, the Debye-Hückel potential with an appropriate effective screening length $\lambda_{\text {eff }}$ is still the best approximation for the grain potential.

If the ion-neutral collision rate is sufficiently high, the potential scaling far from the grain is more like $r^{-1}$ [56].

\section{Trapped Ions}

If an ion loses energy by a collision in the vicinity of the grain potential, the ion can be "trapped" in the potential well and be in a bound state. A self-consistent analytic theory on trapped ions is presented by Lampe et al. [57, 36]. They point out, that trapped ions can dominate the shielding around the dust grain. The density of trapped ions can be of an order of magnitude higher than the density of unbounded ions in the shielding cloud around the grain.

The highest trapped ion density is obtained for $T_{i} \ll T_{e}$, so nearly any ion created in a collision is trapped by the grain potential. A second condition is given by $a^{2} / \lambda_{D}^{2} \ll T_{i} / T_{e}$, that ensures that only a few trapped ions fall immediately on the grain surface.

The presence of trapped ions changes the nature of interaction of the dust grains. In contrast to untrapped ions, the trapped ions contribute to the shielding of the grain charge. Fores due to external electric fields acting on the dust grain are reduced by trapped ions. Furthermore, the inter-grain interaction is weaker and therefore trapped ions can have influence on the 
phase state of a dust particle system.

\section{Dust Grain Potential in an Anisotropic Plasma}

The impact of an anisotropic plasma environment on the charging process is already discussed in section 3.1.2. Not only the grain charge is affected by the streaming plasma, but also the distributions of plasma particles around the dust grain is different. The dust grain potential is not spherical anymore and shows an oscillating wake field with an area of enhanced ion density downstream to the dust grain. This ion focus is responsible for fundamental ordering phenomena observed in several complex plasma experiments [1, 58].

The grain potential can be calculated applying the linear dielectric response formalism. The electrostatic potential associated with a point like charge of $e Z_{d}$ is [59, 30]

$$
\phi(r)=\frac{e Z_{d}}{2 \pi^{2}} \int d^{3} \mathbf{k} \frac{e^{i \mathbf{k} \cdot \mathbf{r}}}{\mathbf{k}^{2} \epsilon(\omega, \mathbf{k})},
$$

where $\epsilon(\omega, \mathbf{k})$ is the plasma dielectric function.

The wake structure of $\phi(r)$ and its dependency on plasma parameters is analyzed by analytical approximations of $\phi(r)$ in [60, 61, 62] and by numerical models in [63, 64, 65]. Some of these works neglect Landau and collisional damping of the wake field by choosing a simple form of the plasma dielectric function $\epsilon(\omega, \mathbf{k})$. This gives only a rough picture of the wake formation, because the damping mechanisms are essential for the amplitude and range of the oscillatory wake field.

Lampe et al. [59] use a more sophisticated approach for $\epsilon(\omega, \mathbf{k})$ and find that the potential depends on three parameters: the Mach number $M=u_{i} / c_{s}$, where $c_{s}$ is the ion sound speed, the ratio $T_{e} / T_{i}$, that controls Landau damping and the ratio of ion mean free path and screening length $l_{i} / \lambda_{D}$, which controls collisional damping.

Figure 3.3 illustrates a contour plot of the potential $\phi(r, z)$ with a Mach number of $M=1.5$ for collisionless ions. The grain position is at $(r=0, z=0)$. Solid curves denote negative potential and dashed lines indicate positive potential. The ions are streaming from the left to the right. The focusing of the ion flow causes a region with positive potential downstream to the grain.

Upstream to the grain and to the side, $\phi(r)$ is close to the Debye-Hückel form (3.22), but with an effective screening length $\lambda_{\text {eff }}$ that depends on the Mach number and the direction. For subsonic ion flows, the ions as well as the electrons contribute to the shielding. In the case of supersonic ions, only electrons are responsible for shielding and the screening length is given by $\lambda_{D e}$.

The oscillatory wake field develops within the Mach cone behind the dust grain. The wavelength of the oscillations is equal to $2 \pi M \lambda_{D e}$ in the supersonic case [61], and the wake is strongest for Mach numbers near 1.

In the linear approach of plasma response, the magnitude of the wake potential is expected 
Figure 3.3: Contour plot of the anisotropic grain potential $\phi(r, z)$ for $M=1.5$ and $\mathrm{Te} / T_{i}=25 \mathrm{in}$ the collisionless case. The grain is at position $(r=0, z=0)$ and the ions are streaming from the left to the right. Solid curves indicate a negative potential and the dashed curves indicate a positive potential. The distances are normalized to the electron Debye length $\lambda_{D e}$. (The graph is taken from Lampe et al. [59].)

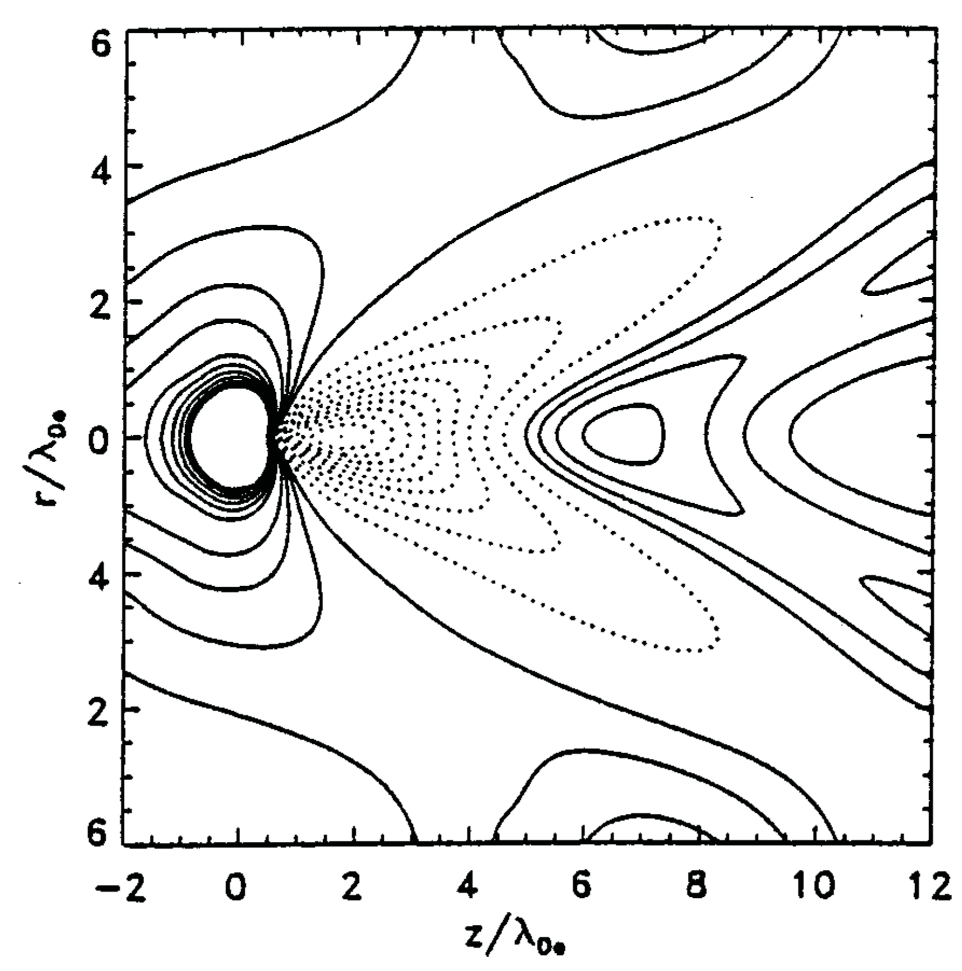

to be proportional to the dust grain charge. Winske et al. [63] have shown, however, that the magnitude is fairly constant at large grain charges. This is attributed to nonlinear effects due to ions that are trapped in the grain potential.

Landau damping and collisions cause a rapid decay of the wake field amplitude. Under typical conditions of a RF-discharge, the wake field is truncated after the first node (first positive potential peak).

The positive potential peak can attract other dust grains downstream. But this attractive force is non-reciprocal, i.e. the upstream particle is not attracted by the downstream particle. The non-reciprocal interaction is an explanation for the alignment of the grains into chains that is observed in experiments [66].

Furthermore, the non-reciprocal interaction is essential for phase transitions and anomalous heating of the dust component. The melting and condensing transition of the dust ensemble are induced by instabilities, that are present due to the non-reciprocal interaction. The expected structures and the instability induced phase transitions are discussed in the section 3.3 in more detail.

\subsubsection{Ion Drag Force}

A dust grain immersed in a flowing plasma experiences a net force in the direction of the flow due to momentum transfer by collisions with ions and electrons. This drag force is negligible for electrons, because of the low electron mass, but the ion drag force plays a crucial role in 
complex plasmas.

The ion drag force is responsible for the void formation [41] and affects the inter-particle interaction and transport mechanisms in a complex plasma. Furthermore, the wave phenomena and the stability of dust structures are determined by the ion drag [29].

Two components contribute to the ion drag force, the collection force and the orbit force. The collection force is due to the momentum transfer by absorption of ions on the grain surface, and the orbit force is associated with the momentum that is transfered by scattering of ions in the grain potential. The general expression for the ion drag force is

$$
F_{i d}=m_{i} \int \mathbf{v} v f_{i}(\mathbf{v})\left[\sigma_{c}+\sigma_{o}\right] d \mathbf{v}
$$

where $\sigma_{c}$ and $\sigma_{o}$ are the cross sections of ion collection and scattering (orbit force), and $f_{i}(\mathbf{v})$ is the velocity distribution of the ions.

The magnitude of the ion drag force depends on the strength of the interaction with the grain potential. This is expressed by the scattering parameter $\beta=Z e^{2} /\left(4 \pi \epsilon_{0} m_{i} v_{i}^{2} \lambda_{D}\right)=$ $\rho_{0} / \lambda_{D}$, where $\rho_{0}$ defines the Coulomb radius.

The derivation of the orbit cross section $\sigma_{o}$ requires the knowledge of the exact interaction potential. In the case of weak interactions between grain and ions $(\beta \ll 1)$, the ions are scattered within the Debye sphere $\left(\rho_{0} \ll \lambda_{D}\right)$. Here, the potential scales as $r^{-1}$ [55] and the well-known Coulomb scattering formalism can be applied.

The calculation of the cross section $\sigma_{o}$ is performed for example by Barnes et al. in [67]. Here, all ions with impact parameters larger than the Debye length are neglected, i.e. $\rho_{\max }=\lambda_{D}$. The orbit cross section in the Coulomb regime is

$$
\sigma_{o}^{\text {Coul }}=4 \pi \rho_{0}^{2} \ln \left(\frac{\lambda_{D}^{2}+\rho_{0}^{2}}{\rho_{c}^{2}+\rho_{0}^{2}}\right)
$$

where $\rho_{c}=a\left(1+2 \rho_{0} / a\right)^{1 / 2}$ is the maximal impact parameter for collection [5][68].

The orbit force in the Coulomb scattering regime is [67]

$$
F_{i d}^{\text {Coul }}=4 \pi n_{i} m_{i} v_{s} \rho_{0}^{2} \ln \left(\frac{\lambda_{D}^{2}+\rho_{0}^{2}}{\rho_{c}^{2}+\rho_{0}^{2}}\right) \mathbf{u}_{\mathbf{i}}
$$

where $v_{s}=\left(u_{i}^{2}+v_{T_{i}}^{2}\right)^{1 / 2}$ and $\mathbf{u}_{\mathbf{i}}$ is the ion streaming velocity.

The approach of Barnes et al. is extended to larger values of $\beta$ by Khrapak et al. in [68, 69]. It is assumed that all ions approaching the grain closer than $\lambda_{D}$ can contribute to the orbit force. The orbit cross section in this approximation is

$$
\sigma_{o}(\beta) \simeq 4 \pi \rho_{0}^{2} \ln \left(1+\frac{1}{\beta}\right)
$$

\footnotetext{
${ }^{5}$ Thus, $\rho_{c}$ is the minimal impact parameter for ion scattering.
} 
This cross section is in good agreement with numerical calculations for scattering parameters up to $\beta \sim 5$.

The second contribution due to ion collection can be described by the OML approach (see section 3.1.1). The cross section for ion collection is given by $\sigma_{c}=\pi \rho_{c}^{2}$ as written in equation (3.4).

The total ion drag force after Khrapak et al. is calculated by the integral in equation (3.24), assuming a shifted Maxwell-Boltzmann velocity distribution. The integration gives [68]

$$
F_{i d}=\frac{8 \sqrt{2 \pi}}{3} a^{2} n_{i} m_{i} v_{T_{i}}\left[1+\frac{e \phi_{s}}{2 m_{i} v_{T_{i}}}+\left(\frac{e \phi_{s}}{2 m_{i} v_{T_{i}}}\right)^{2} \Lambda\right] \mathbf{u}_{\mathbf{i}},
$$

where $Q_{d}=4 \pi \epsilon_{0} \phi_{s}$ is used. The first and second terms of the sum describe the collection ion drag force and the third part is the orbit contribution. $\Lambda$ denotes the modified Coulomb logarithm integrated over the Maxwell-Boltzmann distribution

$$
\Lambda=2 \int_{0}^{\infty} e^{-x} \ln \left[\frac{2 \lambda_{D} x+\rho_{0}\left(v_{T_{i}}\right)}{2 a x+\rho_{0}\left(v_{T_{i}}\right)}\right] d x .
$$

In the regime of strong ion-grain interactions $(\beta \gg 1)$, the Coulomb radius $\rho_{0}$ is much larger than the screening length $\lambda_{D}$. The potential can be described by a Debye-Hückel potential at these distance from the grain. As discussed in 3.1.2, the Debye-Hückel potential shows potential barriers in the effective potential for high $\beta$. This makes the derivation of the orbit cross section more complicated. But, as pointed out in [34], the orbit force can be neglected for large scattering parameters and only the collection ion drag has to be considered.

To derive the collection cross section for $\beta \gg 1$, the OML approach cannot be applied anymore, because of the barriers in the effective potential. Khrapak et al. estimated the collection cross section to be $\sigma=\pi \rho_{*}^{2}$ for large $\beta$, where $\rho_{*}$ is the critical impact parameter defined by

$$
\frac{\rho_{*}}{\lambda_{D}} \simeq \ln \beta+1-\frac{1}{2} \ln ^{-1} \beta
$$

All ions with impact parameters larger than $\rho_{*}$ are reflected by the potential barrier and do not contribute to the collection force. The ion drag force for strong interactions $\beta \gg 1$ is therefore given by

$$
F_{i d}=\frac{8 \sqrt{2 \pi}}{2} \rho_{*}^{2} n_{i} m_{i} v_{T_{i}} \mathbf{u}_{\mathbf{i}} .
$$

This expression should be used for micron-scaled dust grains, because the large grain charge leads to strong ion-grain interactions. For grains of a submicron size, equation (3.28) should be preferred.

The expressions for the orbit ion drag force presented until now, are restricted to subthermal ion flows $\left(M_{T}=u_{i} / v_{T_{i}}<1\right)$. As discussed in section 3.2.1, strong ion fluxes deform the 
grain potential and the assumption of a Debye-Hückel potential is questionable.

In the case of superthermal ion flows $\left(M_{T}>1\right)$, the ion streaming velocity $u_{i}$ should be used to evaluate $\beta$. The value of $\beta=Z e^{2} /\left(4 \pi \epsilon_{0} m_{i} u_{i}^{2} \lambda_{D}\right)$ decreases with the Mach number. For small $\beta$, the Coulomb approach may be used and the orbit ion drag force is given by the expression of Barnes et al., equation (3.26).

For sufficiently high ion streaming velocities $\left(M_{T} \gg 1\right)$, the orbit contribution can be neglected and the ion drag force is given by the collection drag force.

\subsubsection{Other Forces}

\section{Electrostatic Forces}

The electrostatic force is fundamental for ground-based complex plasma experiments using micron-scaled dust particles. The electrostatic force exerted on the dust grains in the parabolic potential of the plasma-wall sheath [42] balances different other forces like gravity and ion drag force. Furthermore, the horizontal particle confinement is realized by additional electric fields, that cause an inward directed electrostatic force.

The electrostatic force in a uniform plasma is given by

$$
\mathbf{F}_{\mathbf{e l}}=Q_{D} \mathbf{E}=-4 \pi \epsilon_{0} a\left(1+a / \lambda_{D}\right) \phi_{s} \mathbf{E} \stackrel{a \ll \lambda_{D}}{\approx}-4 \pi \epsilon_{0} a \phi_{s} \mathbf{E},
$$

where $\mathbf{E}$ denotes the external electric field.

The Debye sheath around the dust grain does not shield the grain from external electric fields. The Debye sheath cannot be assumed to be attached to the dust particle, but it represents only a local perturbation of the plasma density distribution. The Debye sheath is the response of the local plasma to the electric field of the dust particle and the external field [70].

This is different, if trapped ions are present (see section 3.2.1). In this case, an electric force with opposite sign acts on the trapped ion cloud. The total electric force on the dust grain is reduced and the system becomes polarized.

The existence of density gradients in the plasma deforms of the Debye sheath around the dust grain. The density gradient leads to a gradient in the screening length $\nabla \lambda_{D}$ and the sheath gets polarized. Thus, an additional gradient force acts on the grain. An analytical expression is derived by Hamaguchi et al. in [70]:

$$
\mathbf{F}_{\nabla \lambda_{D}}=\frac{Q_{d}^{2}}{8 \pi \epsilon_{0}} \frac{\nabla \lambda_{D}}{\left(\lambda_{D}+a\right)^{2}}
$$

The force $\mathbf{F}_{\nabla \lambda_{D}}$ points in the direction of the decreasing Debye length. Thus, depending on the sign of the grain charge and the direction of the external field, the force $\mathbf{F}_{\nabla \lambda_{D}}$ can enhance or reduce the electrostatic force (equation (3.32)). The electrostatic force in the plasma-wall 
sheath of a RF-discharge is enhanced by the force $\mathbf{F}_{\nabla \lambda_{D}}$, thus it supports the levitation of dust grains.

\section{Gravity Force}

The gravity force on a dust grain with radius $a$ is given by

$$
\mathbf{F}_{\mathbf{g}}=m_{d} \mathbf{g}=\frac{4}{3} \pi a^{3} \rho_{d} \mathbf{g}
$$

where $\rho_{d}$ is the density of the grain material and $\mathbf{g}$ denotes the gravitational acceleration.

The gravity force is less important for nano-scaled dust grains. They are distributed in the bulk of the plasma and filling the whole plasma except for possible voids in the center [41]. If the particle diameter reaches micron size, the gravity becomes important and a levitation in the bulk plasma is not possible anymore.

\section{Gas friction and Neutral Drag Force}

The gas friction exerts a force caused by neutral atoms bombarding the dust grain surface. The friction force is the most important process of energy dissipation of the dust component. The cooling by the background gas increases the coupling between dust particles and this leads to the appearance of ordered structures (plasma crystal).

The gas friction can also be important, if the background gas is streaming relative to the dust particle system. In this case, a neutral drag force is applied to the particle in direction of the gas stream. This can be used to exert stress on dust structures and to generate shear flows [30].

In the limit of large Knudsen numbers $K_{n}=\lambda_{n} / a$, where $\lambda_{n}$ denotes the mean free path for dust-neutral collisions, the neutral drag force can be written as [71]

$$
\mathbf{F}_{\mathbf{n}}=-\delta \frac{4}{3} \pi a^{2} m_{n} n_{n} v_{T_{n}}\left(\mathbf{v}_{\mathbf{d}}-\mathbf{v}_{\mathbf{n}}\right),
$$

where $m_{n}, n_{n}$, and $v_{T_{n}}$ are the mass, number density, and thermal velocity of the neutrals, and $\mathbf{v}_{\mathbf{d}}-\mathbf{v}_{\mathbf{n}}$ denotes the relative velocity between dust component and neutrals.

The parameter $\delta$ depends on how the neutrals are reflected by the particle surface, e.g. for specular reflection $\delta=1$. Measurements of the drag force on Melamine-Formaldehyde dust particles in a complex plasma [72] suggest $\delta=1+\pi / 8$, which, after Epstein [71], stands for diffusive reflection with accommodation.

Using the Epstein friction coefficient $\beta_{E}=8 \delta p /\left(\pi a \rho_{d} v_{T_{n}}\right)$, the neutral drag force can be written as

$$
\mathbf{F}_{\mathbf{n}}=-m_{d} \beta_{E}\left(\mathbf{v}_{\mathbf{d}}-\mathbf{v}_{\mathbf{n}}\right),
$$

where $p$ denotes the gas pressure and $\rho_{d}$ is the mass density of the dust material. The natural drag force depends linearly on the gas pressure and is proportional to the square of the grain 
radius.

\section{Thermophoretic Force}

The thermophoretic force is relevant if temperature gradients are present in the background gas. The larger momentum transfer of the "hotter" neutrals result in a net force in the direction of the colder side. An analytic expression for the thermophoretic force is given by [54]

$$
\mathbf{F}_{\text {th }}=-\frac{32}{15} \frac{a^{2}}{v_{T_{n}}}\left(1+\frac{5 \pi}{32}(1-\alpha)\right) \kappa_{T} \nabla T_{n}
$$

where $\kappa_{T}$ and $T_{n}$ are the thermal conductivity and the temperature of the gas. For grain surface temperatures and gas temperatures below $500 \mathrm{~K}$, the coefficient $\alpha$ can be approximated with $\alpha \approx 1$ and the expression simplifies.

The thermophoretic force is in particular important for nano-scaled dust particles, because the heating of the gas by the discharge can already change the dust distribution significantly. For micron size dust grains, the thermophoretic force can be used to sustain the particle levitation and to form small three-dimensional dust structures (3D Coulomb balls) [73].

\section{Radiation and Photophoretic Force}

The use of lasers to analyze the dust particle structure and dynamics is restricted to relatively low light intensities to avoid perturbations of the dust system. The force due to the laser radiation has two sources. Firstly, the radiation pressure pushes the dust grains in the direction of the beam. This component of the laser force can be written as [28]

$$
F_{\text {rad }}=\gamma \frac{I_{\text {laser }}}{c} \pi a^{2}
$$

where $I_{\text {laser }}$ denotes the intensity of the laser radiation and $c$ is the speed of light. The coefficient $\gamma$ describes the interaction of the photon with the grain surface, $\gamma=1$ stands for pure absorption and $\gamma=2$ describes reflection.

Secondly, the photophoretic force contributes to the laser force. The heating of the illuminated grain surface by the laser leads to a force comparable to the thermophoretic force. The neutral particles, that are reflected on the "hotter" side of the grain, leave with a higher velocity than on the cold side. A net force is exerted on the particle in the direction of the laser beam.

\section{Shadowing Force}

In a uniform plasma environment, the drag force caused by ion and neutral bombardment on an isolated dust grain is zero in average. The situation changes, if a second dust grain is placed in the vicinity to the first one. In this case, a part of the uniform particle flux is blocked and a net force is exerted on the dust grain. This shadowing force leads to an attraction between 
the dust grains and is particularly important for large dust systems [10].

The force depends on the inter-particle distance $\Delta$ and scales as $\Delta^{-2}$. In contrast to the Coulomb force, the shadowing force is not screened by plasma particles and should be dominant on long distances. The shadowing force is proportional to $a^{4}$, thus, it is much more important for micron-scaled dust grains than for nano particles.

\subsection{Dust Structures and Phase Transitions in Complex Plasmas}

In complex plasmas, a remarkable example of self-organization occurs under certain experimental conditions. If the gas friction is sufficiently high, the dust component orders to a regular crystal lattice. In ground-based experiments, particles with a diameter of micron size are trapped in the plasma-wall sheath. The anisotropic plasma conditions in this plasma environment have serious consequences on the structures that are observed. The conditions for ordering and the observed lattice structures are discussed in section 3.3 .1

The variation of plasma parameters like the discharge pressure can be used to force a phase transition. In the melted state, strong self-excited oscillations and unexpected high kinetic energies are observed. In section 3.3.2, possible mechanisms to explain strong heating and phase transitions are presented.

\subsubsection{Structural Ordering of Dust Particles}

The phase state of a system of particles interacting via the Debye-Hückel potential is characterized by two parameters. Firstly, the coupling parameter, that is the ratio of the potential energy between two particles and the average thermal energy

$$
\Gamma=\frac{Q_{d}^{2}}{4 \pi \epsilon_{0} \Delta k_{B} T_{d}}
$$

where $\Delta$ denotes the average inter-particle distance. Secondly, the screening parameter, that is defined as the ratio of inter-particle distance $\Delta$ and screening length $\lambda_{D}$

$$
\kappa=\frac{\Delta}{\lambda_{D}}
$$

The phase state depending on $\Gamma$ and $\kappa$ is illustrated in figure 3.4. This figure summarizes available results from various numerical calculations on Debye-Hückel systems. Three different phases can be distinguished. The solid phase at strong coupling divides into bct 6 structures and $\mathrm{fcc} 7$ structures and for weak coupling the system is in a fluid state.

\footnotetext{
${ }^{6} \mathrm{bcc}$ - body centered cubic

${ }^{7} \mathrm{fcc}$ - face centered cubic
} 


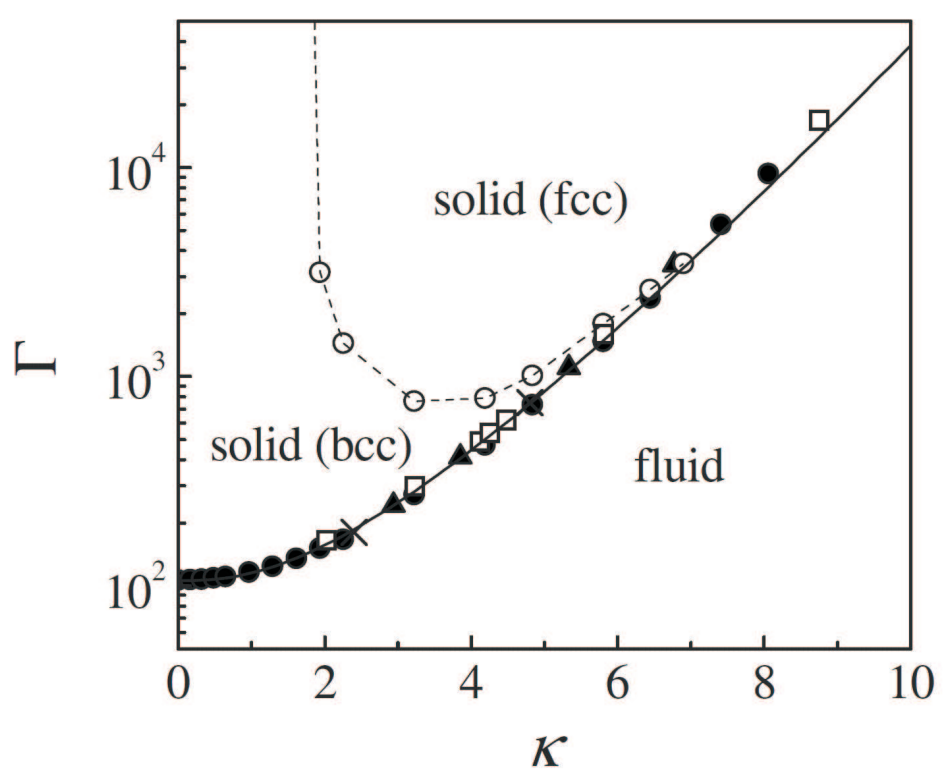

Figure 3.4: Phase diagram of systems with Debye-Hückel interaction. The bcc and fcc crystal structure is observed for strong coupling. Below the melting line (solid line), the system is in a fluid state. The dashed line is the fit to the numerical data judged by eye. The data correspond to: open circles and solid circles Hamaguchi et al. [74]; squares Stevens et al. [75]; triangles - Meijer et al. [76]; crosses - Vaulina et al. [77]. (The figure is taken from Morfill et al. [30].)

The boundary between bcc and fcc was determined by Hamaguchi et al. and the triple point is at $\Gamma=3.47 \cdot 10^{3}$ and $\kappa=6.9$ [74]. The melting line $\Gamma^{*}(\kappa)$ is approximated by Vaulina et al. in [77]:

$$
\Gamma^{*}(\kappa)=106 \frac{\exp (\kappa)}{1+\kappa+\frac{1}{2} \kappa^{2}} .
$$

As discussed in section 3.2.1, the pure Debye-Hückel potential is not valid in the plasmawall sheath, because the ion flow deforms the potential and a wake develops behind the grain. The ions are focused behind the grain and attract dust grains located downstream. As illustrated in figure 3.5, the situation can be described by a simplified picture. The ion focus is replaced by a fictitious particle with positive charge under the restriction of non-reciprocal interaction. That means, the lower dust grain is attracted by the fictitious particle, but there is no force in the opposite direction. The dust grains are aligned into chains due to the attractive force of the fictitious particles. The chains are further stabilized by the horizontal component of the ion drag force.

Here, the fundamental role of the ion drag comes through. In contrast to the prediction of the Debye-Hückel theory, the dust grains can also order into a hexagonal close-packaged (hcp) structure [1, 58]. The bcc and fcc structure is only observed under certain experimental conditions, albeit they are configurations with the lower Madelung energy 8 . The permanent input of energy by the ion flow stabilizes the hcp lattice and demonstrates why complex plasmas are called an energetic open system.

\footnotetext{
${ }^{8}$ The Madelung energy is the average electrostatic energy of a particle in the crystal lattice.
} 


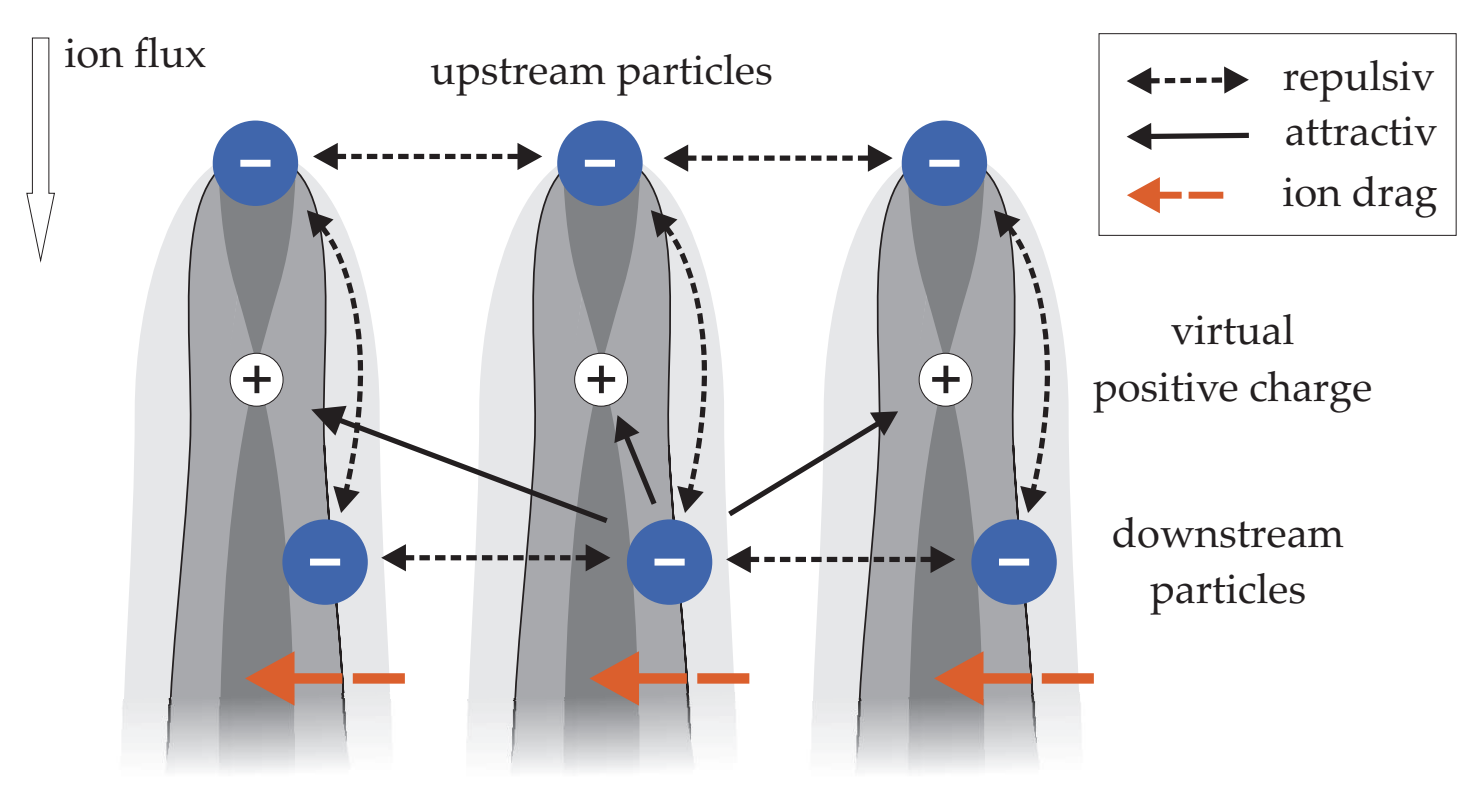

Figure 3.5: Three particles with their wake fields (simplified picture) in an ion flux are shown. Other particles down stream are attracted (dark solid arrows) to the ion focus represented by a fictitious positive charge. The particles tend to align into chains. The horizontal component (perpendicular to the ion flux) of the ion drag force (dashed, single ended arrows) stabilizes the particle chains further.

\subsubsection{Melting and Condensation Transitions of the Dust System}

Figure 3.6illustrates a sequence of images taken from the dust system during a melting transition. The standard CCD technique was used to get single shots of a vertical plane in the dust crystal?. A stable crystal is observed at sufficiently large discharge pressure as shown in figure (a). This image also depicts the arrangement in vertical chains due to the ion focusing. The structure is melted by reducing the pressure. The melting starts at the bottom of the dust system, closer to the lower electrode as shown in (b). At this pressure, the system reveals both phase states at once. The melted part shows self-exited oscillations in the vertical direction with large amplitude. When the pressure is decreased further, the melting front moves upward. At low pressures, the whole system is in a disordered state as depicted in figure (c). The CCD camera system is not capable of following the fast particle motion anymore, and the particle positions cannot be resolved.

The self-exited oscillation as illustrated in figure (b) and (c) is an example for a unique wave phenomenon of complex plasmas, the "dust acoustic wave" (DAW) [78]. If the grain charge fluctuations are neglected, this wave is comparable to the ion acoustic wave, only that the dust acoustic wave is driven by the electrons and the ions and the dust grains provide the inertia. Due to the high mass of the dust grains, the wave frequencies are much lower than for

${ }^{9}$ Visit the experimental section 4.1 for details. 
(a)

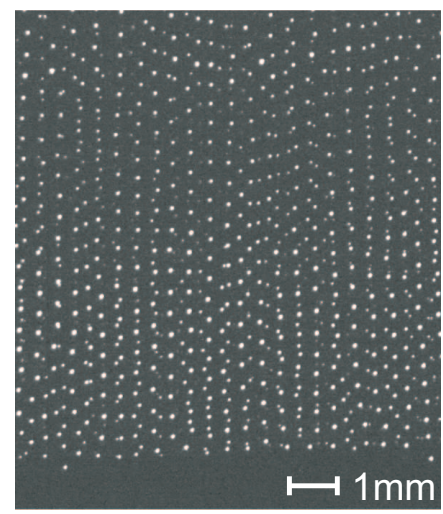

(b)

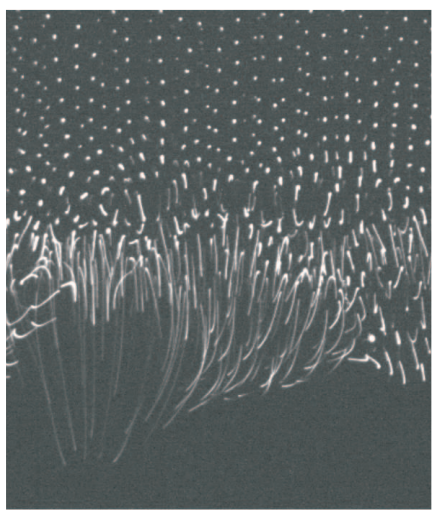

(c)

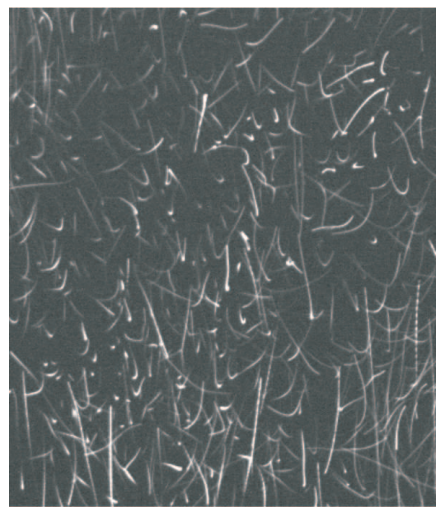

Figure 3.6: A sequence of images demonstrates the melting transition in a complex plasma. The standard CCD technique was used to get single shots of a vertical plane in the dust crystal. In (a), the system is in a well ordered state revealing arrangement in vertical chains due to the ion focusing. In (b), the discharge pressure is reduced and the melting starts at the bottom of the system. In the melted state, the dust grains show self-exited oscillations in the vertical direction. In (c), the whole system is in the disordered state at low pressure.

ion acoustic waves. The frequencies are typically of the order of tens of $\mathrm{Hz}$ and comparable to the dust plasma frequency $\omega_{d}=\sqrt{Z_{d}^{2} e^{2} n_{d} /\left(\epsilon_{0} m_{d}\right)}$.

A remarkable property of the disordered state is the unexpected high thermal energy of the dust grains. At very low pressures, the dust grain can reach energies of the order of $1000 \mathrm{eV}$ which corresponds to a thermal velocity of about $11 \mathrm{~cm} / \mathrm{s}$ for particles with $3 \mu \mathrm{m}$ diameter 10 . This phenomenon is denoted as "anomalous heating" in literature. Several mechanisms are suggested to explain the anomalous heating.

The increase of the thermal energy of the dust component by stochastic charge fluctuations, caused by the discreteness of the charging process, is discussed briefly in section 3.1.4. The increase of energy is of the order of a few $\mathrm{eV}$, thus the stochastically fluctuating charges are a minor contribution to the anomalous heating [29].

If the charge depends on the coordinate of the grain, e.g. in inhomogeneous plasma environments, another contribution to anomalous heating becomes important. Because of the spatial variation of the grain charge, the total energy is not conserved in dust-dust collisions and the mean kinetic energy increases with time [79, 80, 81]. Another facet of this heating mechanism is given by the finite charging time. The self-exited vertical oscillations (figure 3.6 (b) and (c)) can be enhanced by the delayed charging of the dust grains, i.e. a grain moves faster than it can achieve the equilibrium charge determined by the local plasma conditions. The particle gains energy in the electrostatic potential of the plasma-wall sheath, because the charge on the way down is more negative than on the way up [82].

\footnotetext{
${ }^{10}$ These values are related to Melamine-Formaldehyde (MF) particles with a density of $\rho_{M F}=1.51 \mathrm{~g} / \mathrm{cm}^{3}$.
} 
The non-reciprocal interaction between two dust particles via the wake field is another source of anomalous heating. The dynamics of the particles system is studied using a onedimensional approach by Melzer et al. in [5]. In this work, the simplified picture assuming fictitious positive charges as described in figure 3.5 is used. A system of two dust layers is examined, where only the horizontal movement is considered. The coupling between the dust grain is assumed to be linear. The non-reciprocal attractive force is included by a lower coupling constant within the lower crystal layer.

A similar approach is followed by Schweigert et al. in [6], but with nonlinear interaction using the Debye-Hückel potential. In [83], an infinite dust cloud in three dimensions is analyzed.

All these works show, the non-reciprocal particle interaction is not only responsible for the anisotropy of the lattice structure, but also makes the crystal prone to lattice instabilities (phonons). By reduction of the neutral gas friction, the lattice instabilities grow to large amplitudes and induce the melting transition and lead to the strong increase of the thermal energy.

A more comprehensive approach to explain anomalous heating caused by the wake field is described by Joyce and Ganguli et al. [7, 8, 9]. They consider the dynamically shielded potential as described in section 3.2.1 (equation (3.23)), that is obtained by applying linear response theory.

They show analytically that an ion-grain two-stream instability is present at low pressures, and that it is responsible for the anomalous heating of the dust particles. The growth of the instability can be damped by the combined effects of ion-neutral and dust-neutral collisions. An increase of the discharge pressure above a critical value $p_{\text {cond }}$ leads to stabilization of the instability. Thus, the ion two-stream instability not only explains the anomalous heating at low pressures, but also gives the mechanism that triggers the condensation transition to a strongly coupled state. To understand the condensation transition, a closer look on the theory and predictions of ion two-stream instabilities is advisable.

The theoretical picture described in [7, 8, 9] only treats the dust grains as particles, whereas the electrons and ions only appear as a dielectric media. By doing so, the theory and regarded models simplify, because the very fast time scales of electrons and ions are eliminated. This assumption is based on the weak coupling between electrons or ions and dust grains.

In the melted state, where the dust is only weakly coupled, the dispersion relation can be written as

$$
0=1+\frac{1}{k^{2} \lambda_{D e}^{2}}-\frac{\omega_{d}^{2}}{\omega\left(\omega+i v_{d}\right)}-\frac{\omega_{i}^{2}}{\left(\omega-\mathbf{k} \cdot \mathbf{u}_{\mathbf{i}}\right)\left(\omega+\mathbf{k} \cdot \mathbf{u}_{\mathbf{i}}+i v_{i}\right)}
$$

where $\lambda_{D e}$ is the Debye length, $\omega_{d}$ and $\omega_{i}$ denote the dust and ion plasma frequencies, $\mathbf{u}_{\mathbf{i}}$ is the ion stream velocity toward the electrode, and $v_{d}$ and $v_{i}$ are the dust-neutral and ion-neutral collision frequencies. 


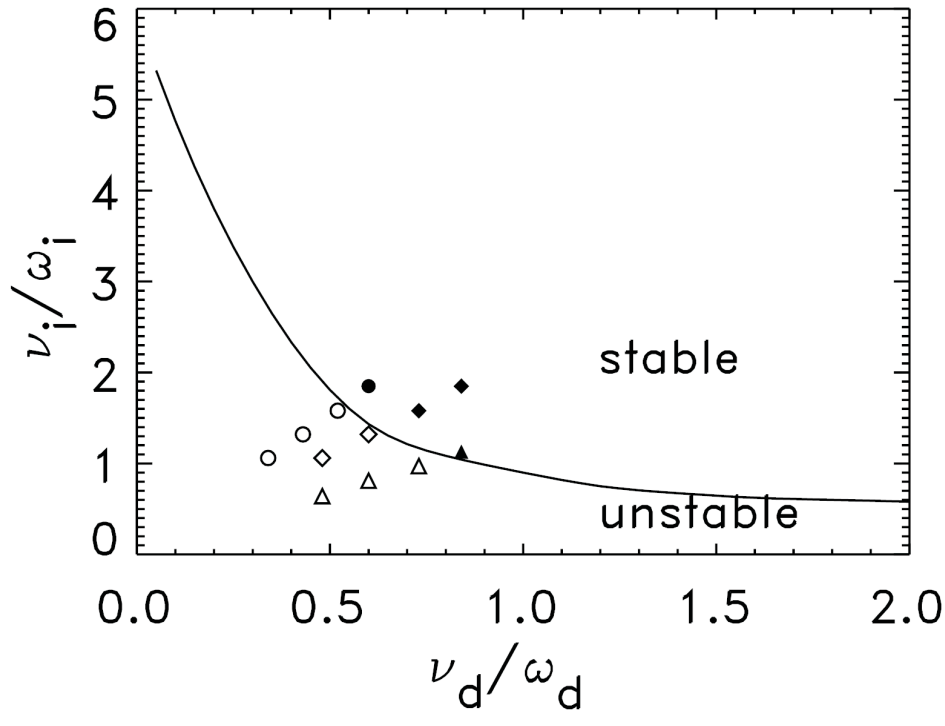

Figure 3.7: Stability boundary in the $v_{d} / \omega_{d}-v_{i} / \omega_{i}$ plane. For a given stability boundary $v_{d} / \omega_{d}$ and $v_{i} / \omega_{i}$ have to be large enough to enter the stable region. This can be achieved for example by increasing the discharge pressure. The points represent simulations of an infinite dust cloud $M=1.0$ (diamonds), $M=0.5$ (triangles) and a simulation of a dust cloud with only six layers and $M=1.0$ (circles). (The figure is taken from Joyce et al. [7].)

Numerical solutions of equation (3.42) show, that all instability modes are stabilized, if the ratios $v_{d} / \omega_{d}$ and $v_{i} / \omega_{i}$ are large enough. For the specific case of $v_{d} / \omega_{d}=v_{i} / \omega_{i}$, a stability condition can be obtained analytically

$$
\frac{v_{d}}{\omega_{d}}=\frac{v_{i}}{\omega_{i}}>\sqrt{2}-\frac{M \cos \theta}{1+\omega_{d} / \omega_{i}}
$$

where $M \equiv u / c_{s}$ is the Mach-number with $c_{s} \equiv \sqrt{k_{B} T_{e} / m_{i}}$ the ion sound speed and $\theta$ is the angle between $\mathbf{k}$ and $\mathbf{u}_{\mathbf{i}}$.

The collision frequency for dust-neutral collisions $v_{d}$ is given by the Epstein formula11 [71]

$$
v_{d}=\delta \frac{8 \sqrt{2 \pi}}{3} \frac{m_{n}}{m_{d}} a^{2} n_{n} v_{T_{n}}
$$

where $m_{n}, n_{n}$ are the mass and density of the neutrals, respectively.

The numerical factor $\delta$ depends on how the molecules or atoms are reflected from the surface of the particle. As mentioned in section 3.2.3, a value of $\delta=1+\pi / 8$ is recommended by experiments on complex plasmas [72].

Equation (3.44) shows, the dust-neutral collision frequency is proportional to the discharge pressure $p$. This is also true for the ion-neutral collision frequency $v_{i}$ that can be written as

$$
v_{i}=C_{i} u_{i} p
$$

where $C_{i}$ is the ion collision coefficient depending on the ion species and $u_{i}$ is the ion streaming speed. The dust plasma frequency is given by

\footnotetext{
${ }^{11}$ In the paper of Epstein [71] the average velocity $\bar{c}$ is used $\left(\bar{c}=\sqrt{8 / \pi} v_{T_{n}}\right)$.
} 


$$
\omega_{d}=\sqrt{\frac{Z_{d}^{2} e^{2} n_{d}}{\epsilon_{o} m_{d}}},
$$

where $Z, m_{d}$, and $n_{d}$ are the charge number, mass, and number density of the dust. The particle mass $m_{d}$ is known for the dust particles used in this work. The dust charge is proportional to the electron temperature and the particle radius $Z \propto T_{e} a$.

The ion plasma frequency is given by

$$
\omega_{i}=\sqrt{\frac{e^{2} n_{i}}{\epsilon_{o} m_{i}}}
$$

with $m_{i}$ the mass of the ions and $n_{i}$ the ion density.

The stabilization of the ion two-stream instability is determined by a stability condition like equation (3.43). Figure 3.7 shows the result of a simulation for a specific set of parameters (see [7] for details). The boundary between the stable and unstable region is given by the right hand side of equation (3.43). To enter the stable region the ratios $v_{i} / \omega_{i}$ and $v_{d} / \omega_{d}$ have to be sufficiently large, e.g. by choosing a sufficiently high gas pressure. The points drawn in figure 3.7 represent simulations of dust clouds with different plasma parameters. Triangles and diamonds represent an infinite dust cloud with Mach numbers $M=1.0$ (diamonds) and $M=0.5$ (triangles).

The circles correspond to a simulation of only six horizontal layers of dust particles. Even though the theory strictly applies only to an infinite dust cloud, a good qualitative agreement with the theoretical predictions was found in this simulation.

The simulations show a finite dust cloud condensates at a different critical pressure $p_{\text {cond }}$ because of boundary effects. An interesting aspect is the convective nature of the ion twostream instability. The extent of dust heating rises with thickness of the dust cloud. This is a significant result regarding to real experiments, inasmuch as the thickness of the dust cloud is limited by the sheath width and therefore is affected by external discharge parameters.

The stabilization of the instability and therefore the condensation of the dust cloud caused by a change of experimental parameters can now be interpreted in terms of the plasma frequencies $\omega_{d}$ and $\omega_{i}$, the collision frequencies $v_{i}$ and $v_{d}$, the Mach number, and the angle $\theta$ between $\mathbf{k}$ and $\mathbf{u}_{\mathbf{i}}$.

The melting transition has also been analyzed by Ganguli and Joyce et al. [7, 8, 9]. In difference to the simplified heuristic models by Melzer and Schweigert as discussed above, they used the correct representation of the wake field potential in the ion flow.

Their simulations show, longitudinal, shear and mixed modes can be driven unstable during the melting transition. The mode, that initiates the melting transition, is believed to be a single shear mode with wave vector $\mathbf{k}$ parallel to the ion flow. That means, the crystal layers oscillate in the horizontal plane relative to each other. This phonon instability can be stabilized at high pressures by the dust-neutral collisions $\left(v_{d} / \omega_{d}\right)$ or by ion-neutral collisions 


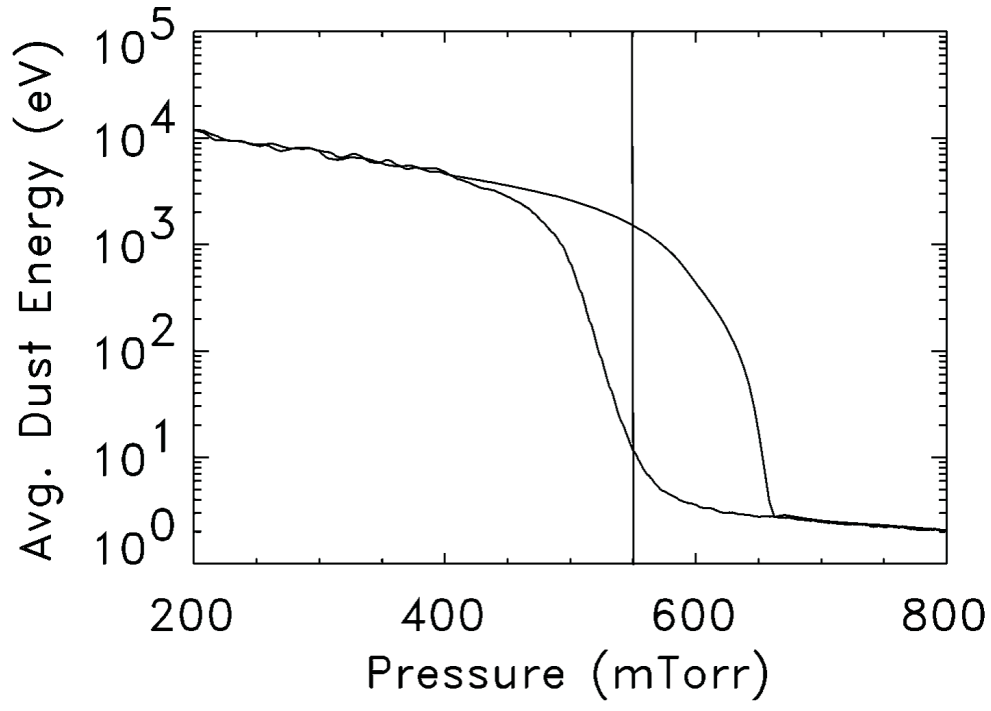

Figure 3.8: Average dust particle energy during a variation of the discharge pressure. The pressure was first increased and a sudden drop of the average energy indicates condensation at a critical pressure $p_{\text {cond }}$. A subsequent decrease of the pressure leads to melting at a critical pressure $p_{\text {melt }}$. The critical pressure for melting $p_{\text {melt }}$ is substantially less than the critical pressure for condensation $p_{\text {cond }}$. (The figure is taken from Lampe et al. [9].)

$\left(v_{i} / \omega_{i}\right)[8]$.

The phonon instability, that grows in the crystal phase, has to be distinguished from the ion two-stream instability in the gaseous phase state. There is no reason why the critical pressured, where the instabilities are stabilized, are the same for both mechanisms. Indeed, the simulations described in [7, 8, 9] show a hysteresis loop, i.e. the critical pressure for melting $p_{\text {melt }}$ is lower than the critical pressure for condensation $p_{\text {cond }}$.

Figure 3.8 depicts the average dust energy obtained from a simulation. First, the discharge pressure is slowly increased and a condensation transition occurs, where the average energy drops from $10^{4} \mathrm{eV}$ to $2 \mathrm{eV}$. This is followed by a decrease of the discharge pressure which causes a melting transition ending up at $10^{4} \mathrm{eV}$ again. As can be seen, the critical pressures $p_{\text {melt }}$ and $p_{\text {cond }}$ are separated by about $130 \mathrm{mTorr}(\approx 17 \mathrm{~Pa})$. 


\section{Chapter 4}

\section{Experimental Setup}

This chapter introduces into the experimental setup of the complex plasma experiment, the standard CCD diagnostic, and the DLS diagnostic.

The first section 4.1 presents the discharge chamber and treats the injection and confinement of dust particles in the plasma.

Section 4.2 introduces the CCD observation technique. After a presentation of the components of this diagnostic and their arrangement, the video processing and data analysis are described shortly.

The last section 4.3 concerns the DLS diagnostic. First, the scattering geometry is introduced and the components required for the scattering experiment are presented. Then, the imperfections of these components are discussed and a validation of the setup based on photon statistics of unscattered laser light is shown. The application of the Siegert relation to derive the homodyne scattering function is justified by analysis of photon statistics of scattered laser light. Finally, the data acquisition and analysis of the DLS approach is discussed.

\subsection{Complex Plasma Experiment}

A cross section of the plasma chamber and the experimental devices required to run the discharge are shown in figure 4.1. A capacitively coupled radio frequency argon discharge is driven between two plane parallel aluminum plates (5). To ignite and sustain the discharge, a radio frequency (RF) generator 12 (1) with working frequency of $13.56 \mathrm{MHz}$ is connected through a matching box to the upper electrode (2). The forward and reflected power is monitored with a power meter 13 . The forward power is of the order of $3 \mathrm{~W}$ with a standing wave ratio below 2 .

The dust particles are immersed into the plasma by a dust dispenser (4). This is a small stainless steel cylinder with a metal mesh at the bottom. The cylinder contains dust powder and is placed above a hole in the upper electrode. If the cylinder is shaken by a vibrating motor, the dust is falling into the plasma.

\footnotetext{
${ }^{12}$ Power generator Cesar 133, 13.56 MHz, Dressler HF-Technik

${ }^{13}$ Power reflection meter NAP 392.4017.02, Rohde \& Schwarz
} 


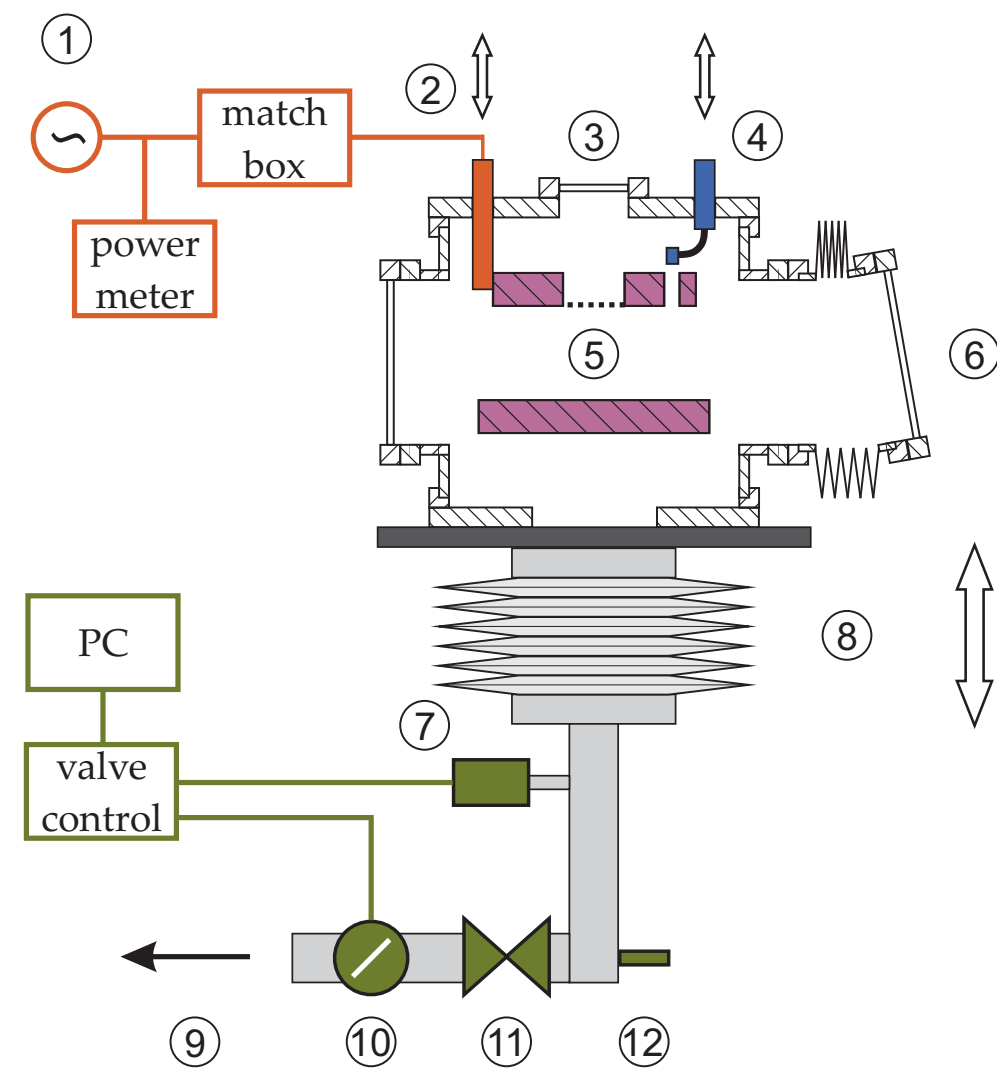

Figure 4.1: Scheme of the complex plasma experiment: (1) RFgenerator (2) movable, powered electrode (3) window $\varnothing 32 \mathrm{~mm}$ (4) movable dust dispenser (5) electrodes, plasma, and dust particle system (6) tilted window (7) capacitance manometer (8) flexible bellow and mechanical drive to move chamber vertically (9) turbo molecular pump and scroll pump (10) automatic butter fly valve (11) mechanical valve (fixed) (12) gas inlet and flow control.

The dust particles are spherical Melamine-Formaldehyde particles with diameters of $3.24 \mu \mathrm{m}$ and $7.23 \mu \mathrm{m}$. They have a monodisperse size distribution with a statistical deviation of only $\pm 0.09 \mu \mathrm{m}$.

The dust dispenser as well as the upper electrode are movable in vertical direction. This allows to modify the distance between the electrodes. For a light scattering experiment in the vertical plane, the electrode distance needs to be sufficiently large to avoid scattering at the electrodes. On the other hand, the electrode distance determines the amount of dust that can be trapped and the distribution of dust in the chamber. According to this, the electrode distance was optimized to be $54 \mathrm{~mm}$. The diameters of the upper electrode and the lower electrode are $120 \mathrm{~mm}$ and $140 \mathrm{~mm}$, respectively.

The dust system can be observed from the top through the window (3) and a hole in the upper electrode. This hole is covered with a grounded metal mesh to ensure a homogeneous plasma. The observation from the side is possible through four windows with diameters of $100 \mathrm{~mm}$. One window (6) is mounted on a flexible bellow and can be tilted to reduce backward scattering from the DLS laser into the dust system. This is in particular important for small angle scattering experiments.

The whole plasma chamber is mounted on another flexible bellow (8). This allows to move the chamber relative to the table in vertical direction. A precise mechanical drive ensures sta-

\footnotetext{
${ }^{14}$ In the following, the particles are denoted as $3 \mu \mathrm{m}$ and $7 \mu \mathrm{m}$ particles.
} 
Figure 4.2: Scheme of the matching circuit (left, dashed box) and the low pass filter (right, dashed box). The matching circuit matches the impedance of the discharge chamber to the output impedance of the generator. The discharge is capacitively coupled through a blocking capacitor. The low pass filter is implemented to apply DC voltages between $-40 \mathrm{~V}$ and $40 \mathrm{~V}$ on the powered segment of the upper electrode.

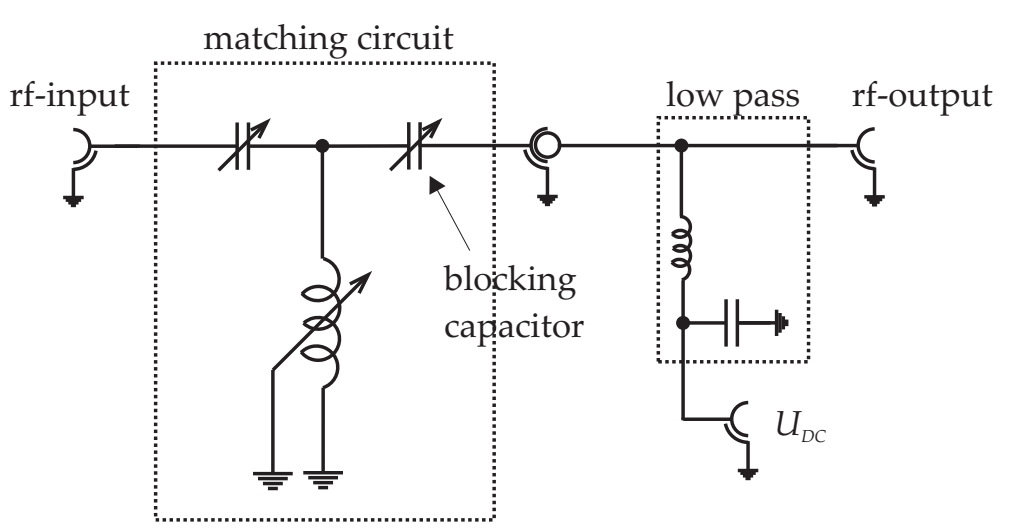

bility of the plasma chamber and reduces the vibrations during the movement of the chamber. This mechanism can be employed to move the dust particles cloud relative to the laser beam of the DLS system, that is mounted on the table. The vertical position of the chamber is measured precisely relative to the table.

A capacitance manometer 15 (7) and a butter fly valve 16 (10) are used to monitor and regulate the discharge pressure. Both devices are controlled by the valve control unit 17 via a computer program written in Visual Basic. The valve control unit can automatically adjust and hold a specific pressure. The pressure can be measured with a repetition time of $0.02 \mathrm{~s}$.

The gas flow is introduced at (12), far away from the discharge chamber. This minimizes the turbulences in the chamber and the dust ensemble is not disturbed by streaming background gas. The gas flow is between $2 \mathrm{sccm} 18$ and $5 \mathrm{sccm}$ and is regulated by a mass flow controller 19 . A turbo molecular pump 20 in combination with a scroll pump21 maintains the vacuum (9). The scroll pump reaches a minimal pressures of $10 \mathrm{~Pa}$, which is not low enough to study the highly melted state of the dust system near $1 \mathrm{~Pa}$. To work with the turbo molecular pump, the cross section of the pipe has to be reduced with a mechanical valve (11). Otherwise, the automatic valve is not capable of stabilizing the pressure and strong valve movements disturb the dust system.

A scheme of the matching box is illustrated in figure 4.2 It consists of two parts. Firstly, a matching network 22 that matches the impedance of the plasma discharge to the output impedance of the generator. This configuration is an example for capacitively coupled plasma (CCP) discharge. It consists of a low pass and a blocking capacitor. Secondly, a low pass is

\footnotetext{
${ }^{15}$ Baratron type $627 \mathrm{~A}$, MKS Instruments

${ }^{16}$ Throttling valve type 253B, MKS Instruments

${ }^{17}$ Pressure controller 600 series, MKS Instruments

${ }^{18} \mathrm{sccm}$ - standard cubic centimeter per minute

${ }^{19}$ Mass flow controller (10 sccm) type 1179 and 4 channel readout type 247D, MKS Instruments

${ }^{20}$ TurboDrag pump TMU 261 and DCU control unit, Pfeiffer Vacuum

${ }^{21}$ Dry scroll pump IDP-3, Varian

${ }^{22}$ Watt antenna tuner AT2K 2000, Palstar
} 
(a)

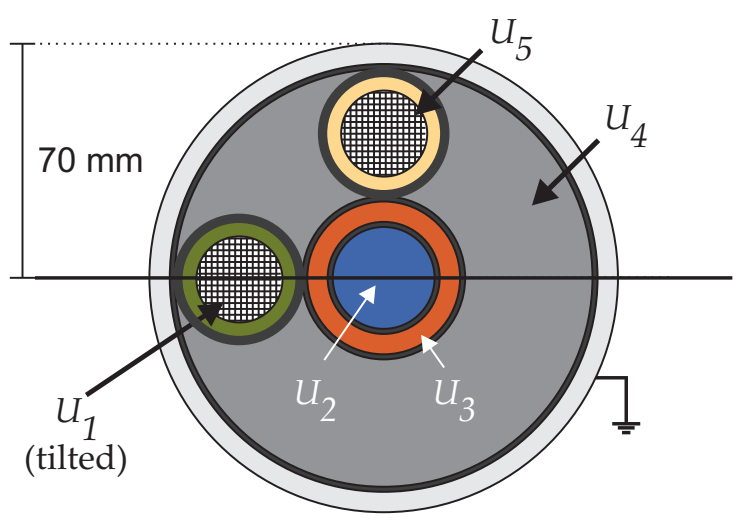

(b)

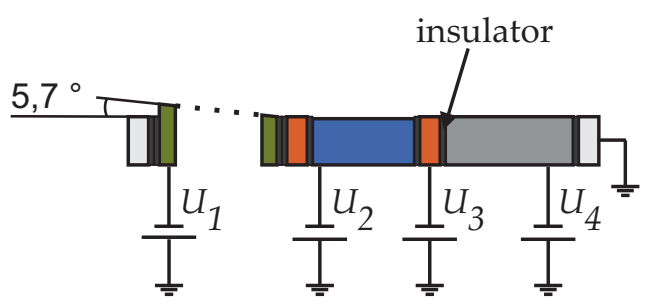

Figure 4.3: Top view (a) and cross section (b) of the lower electrode. Voltages can be applied to five segments $U_{1}$ to $U_{5}$ independently by a multichannel, decoupled voltage supply. The voltages are typically between $-20 \mathrm{~V}$ and $20 \mathrm{~V}$. The space between the segments if filled with an insulator. The outer edge of the electrode is grounded.

used to apply a DC voltage on the powered segment of the upper electrode. This DC voltage is an important parameter to manipulate the dynamics and structures of the dust particles and to vary the vertical position of the dust cloud. In this work, DC voltages between $-40 \mathrm{~V}$ and $40 \mathrm{~V}$ are applied.

The lower electrode is designed for particle confinement. The vertical confinement is realized by the electric field in the plasma-wall sheath. For the horizontal confinement, additional electric fields are necessary. In complex plasma experiments, this is most commonly realized by an outer ring on a higher potential that forms a potential well. The lower electrode in this experiment has five segments to manipulate the dust cloud and to study transport mechanisms of complex plasmas. A positive or negative voltage can be applied to each segment independently by a multichannel, decoupled voltage supply.

Figure 4.3 shows a top view (a) and a cross section (b) of the lower electrode. The space between the segments is filled with a temperature-resistant insulator. The segment denoted with $U_{1}$ is just below the dust dispenser. The dust that is not trapped in the plasma falls through a metal mesh and is collected in a small bin below the electrode. As shown in (b), this mesh is tilted by $5.7^{\circ}$ to push the trapped particles toward the center of the discharge.

The segment denoted with $U_{5}$ also has a metal mesh with a small bin below it. The dust can be transported to this segment by variation of the voltages. Then the dust can be removed from the plasma leaving a clean electrode for the next experiment.

During the experiment, the same potential is applied to the segments $U_{1}, U_{4}$, and $U_{5}$. Hence, they act like a single outer confinement ring. In combination with $U_{2}$ and $U_{3}$, a potential well is formed. The applied voltages are between $-20 \mathrm{~V}$ and $20 \mathrm{~V}$.

Particle trapping is also possible, if all voltages are set to zero. Here, the outer edge 
top view

Figure 4.4: Experimental setup of the CCD diagnostic. A laser is focused to a sheet by an inbuilt lens system. The scattered light of the dust particles within the sheet is detected by a CCD system. The CCD system consists of a CCD camera and a macro lens system, and is mounted perpendicular to the laser sheet. The observation of a vertical plane is shown. A hole in the upper electrode allows to observe horizontal planes too (top view).

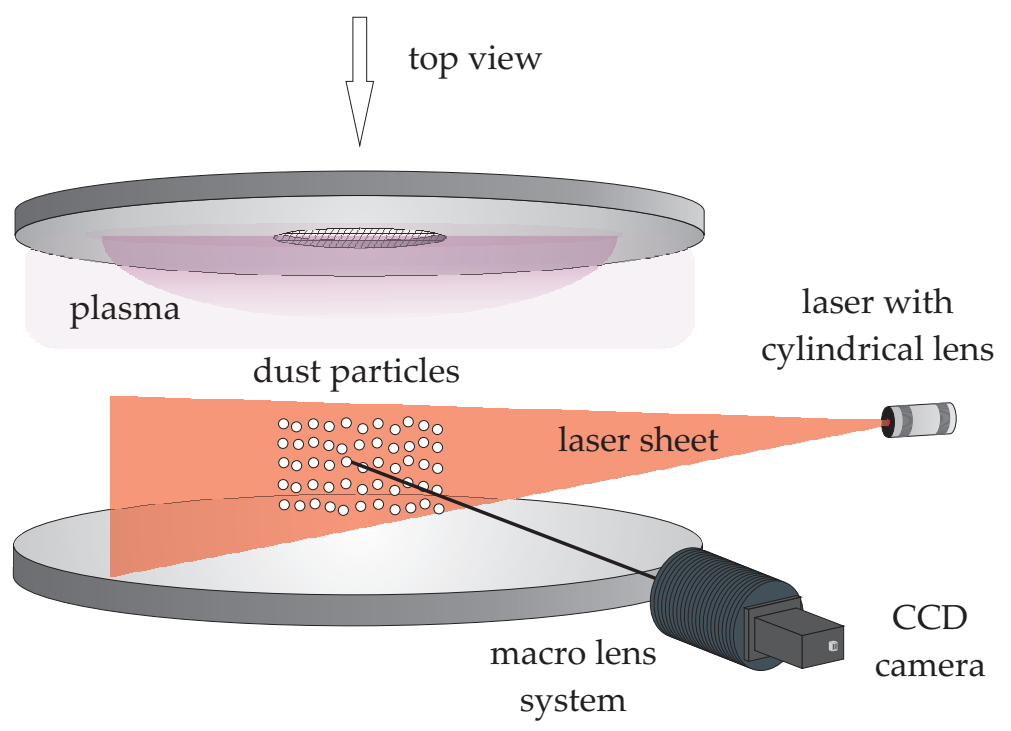

(grounded) of the electrode provide the potential well. Furthermore, the central segment $U_{2}$ is slightly lower relative to the other segments, which further sustains the particle confinement.

\subsection{CCD Observation Technique}

In contrast to other strongly coupled systems, complex plasmas offer a unique way of particle observation. The particle system can be studied directly on the kinetic level, because the micrometer grains can be seen with the naked eye. This is used in the CCD observation technique, where the particles are illuminated by a laser and detected by means of a CCD camera. The laser beam is focused to a sheet by a cylindrical lens system. Thus, the CCD technique delivers information only about a 2D plane. The details of the CCD setup is presented in section 4.2 .1

The video data are analyzed by a video processing software that is discussed shortly in section 4.2.2. The trajectories of individual particles are obtained and analyzed regarding the dynamics and structure of the dust system. The studies of the particle movement is limited by the spatial and temporal resolution of the CCD camera.

The CCD setup and the analysis software is described in full detail in [84].

\subsubsection{CCD Setup}

The principle of the CCD technique as applied in this work is illustrated in figure 4.4, A solid-state laser 23 is used for illumination of the dust particles. The particle movement is detected by a CCD camera system that is mounted perpendicular to the laser sheet. Here, the observation of a vertical plane of the dust system is shown. The observation of a horizontal

\footnotetext{
${ }^{23}$ Line generator type 25CM-660-40-M02-A8-2 and lens type 5LM-8-S325-L, Schäfter+Kirchhoff
} 
plane (top view) is possible through the hole in the upper electrode with a horizontal laser sheet.

The laser has a wavelength of $682 \mathrm{~nm}$ and an output power of $P \leq 40 \mathrm{~mW}$. An inbuilt lens system forms the laser sheet. The focal length can be adjusted manually. The sheet thickness varies only a little within three centimeters around the focus and is about $150 \mu \mathrm{m}$.

The camera system consists of a CCD camera24 and a lens system. The CCD chip has a resolution of 1280 pixel $\times 1024$ pixel with a pixel size of $6.7 \mu \mathrm{m} \times 6.7 \mu \mathrm{m}$. The acquisition rate (frame rate) of the camera is 24 frames/s at full resolution and can be increased up to $500 \mathrm{frames} / \mathrm{s}$, if the resolution is reduced. The lens system is a macro len 25 that resolves the individual particles on sub-micrometer scale.

The transformation from pixel to real distances requires the knowledge of the scale function $f_{s}(D)$, that depends exponentially on the focal distance $D$. The function $f_{s}(D)$ is determined in [84] with an error below $2 \%$ in the relevant range.

\subsubsection{Video Analysis Software}

The analysis of the video data is performed with the interactive data language (IDL)26. The data analysis routines are based on the IDL project "Spit" developed by the group of Prof. G.E. Morfil|27. The routines were refined in several ways in a previous work [84]: A graphical user interface (GUI) was developed to simplify the handling of the routines; The hierarchical data format (HDF) 28 was introduced to keep all parameters of the analysis and the corresponding data together in an organized file; The direct processing of video files of different formats instead of reading sequences of images were enabled.

The first step of the data analysis is to convert the video files into a sequence of arrays containing the intensities of the pixels. This is done by an IDL packaged written by O. Kornilov 29 that manages the connection to the Video for Windows interface. These routines were implemented into the GUI procedure.

The video material is optimized during this conversion process. Several filters are implemented to improve the contrast and brightness of the images and to smooth pixel errors. The Avisynth frame server 30 is implemented for the video processing. This frame server gives access to various open source video processing routines and the program can be easily extended by additional filters.

The next step is to identify the dust particles in the intensity arrays. All spots with intensities above a certain threshold are assumed to represent a dust grain. The selection is refined

\footnotetext{
${ }^{24}$ CCD camera type A622f, Basler Vision Technologies

${ }^{25}$ Macro lens Af Micro-Nikkor, $200 \mathrm{~mm}$ focal length, Nikon

${ }^{26}$ http://www.ittvis.com/

${ }^{27}$ Max-Planck-Institut für extraterrestrische Physik Garching, www .mpe.mpg.de

${ }^{28}$ http://www.hdfgroup.org

${ }^{29}$ http://www.kilvarock.com/freesoftware/dlms/avi.htm

${ }^{30}$ http://avisynth.org
} 
by neglecting all spots with a radius smaller than a certain value, e.g. single pixels with high intensities are neglected. The coordinates of a particle are given by the geometric center of the spot weighted by the intensity profile. This only works, if the particles appear as round spots. If the exposure time is too long to resolve the particle movement, the particles appear as strings and a correct determination of the particle coordinates fails. Thus, the acquisition frame rate has to be sufficiently high.

The GUI procedure simplifies the determination of the best intensity threshold by displaying the video frames and all identified particles. The user can easily find the best result by variation of the threshold value.

The dynamical information of the dust grains is obtained by tracking the particles through the sequence of frames. A particle at position $(x, y)$ in frame $A$ has to be allocated to a particle in the following frame $B$. The simplest approach is to allocate the particle closest to $(x, y)$ in frame $B$ to the original particle. This approach fails, if the original particle moves too fast (or the video frame rate is too low) or if it is not visible anymore, because it left the laser sheet. In these cases, another particle will be closer to $(x, y)$ and the allocation will be wrong.

The approach can be improved by choosing an area around $(x, y)$, in which the particle has to be found. If no particle is in the area, the particle is assumed to be lost. The area should not be too small, because then the velocity distribution gets truncated at fast velocities. This method works well, if the frame rate is sufficiently high.

It can be further improved by using the velocity information of the previous frames to make a guess of the new particle position. The particle is searched within an area around this guessed position. This is in particular effective for streaming particle systems.

The data obtained by this procedure contain the full structural and dynamical information of the particles in the two-dimensional plane. In the following analysis steps, the particle density, velocity distribution, and average thermal energy of the particles are calculated. The structure of crystal lattice is analyzed by the pair-correlation function or the angular correlation function [28]. The defect distribution in the crystal lattice can be studied by construction of the Voronoi diagram and counting the number of neighbors for each particle [28].

A standard CCD system only works well in the ordered phase state of the dust system. As mentioned before, the frame rate is critical to find the correct particle coordinates and to ensure right particle tracking. The effect of the frame rate on the measurement of the thermal particle energy is described in a conference contribution of Statsenko et al. [85]. It turns out that a frame rate of the order of 50 frames/s is necessary to obtain trustful results of the particle energy in the ordered state. The experimental determination of the fast particle movement in the melted state is not easily realized. Expensive, high speed cameras are required to examine this phase state by the CCD approach. 


\subsection{DLS Observation Technique}

The DLS setup presented here is designed to examine the dust particle motion in horizontal and vertical direction at the same time. In common DLS experiments, this is realized by two independent laser beams. There, the lasers are arranged in a way, that the scattering vectors $\mathbf{q}_{1}$ and $\mathbf{q}_{2}$ point into the horizontal and vertical direction.

In this work, a homodyne DLS setup with a single incident laser beam is proposed to measure both components. The laser beam has a circular polarization. The radiation with polarization perpendicular to the electrode is selected to study the particle movement in the horizontal plane and the parallel polarization component is used to analyze the vertical particle movement.

The single laser approach simplifies the DLS setup and is proposed in this work for the first time. Compared to the common approach, the number of mirrors, lenses, and polarizers is reduced, which is advantageous for the costs of the experiment. Furthermore, arrangement and adjustment of the laser system is simplified and a significant source of error is eliminated by omitting the second laser.

The detailed setup of the single laser approach is presented in the following section 4.3.1. The sources of imperfection in a DLS experiment are discussed and the validity of the setup is proven in section 4.3.2. Finally, the data acquisition and the analysis software is introduced shortly in section 4.3 .3 .

\subsubsection{DLS Setup}

Figure 4.5 shows a scheme of the homodyne DLS setup: The light of a Helium-Neon 31 (1) laser is scattered by the dust grain ensemble (6) and is detected by two fast photomultipliers (9) and (10).

The laser has a wavelength of $632.8 \mathrm{~nm}$ with a linear polarization and an output power of 21 $\mathrm{mW}$. The laser beam is attenuated as much as possible, to avoid heating of the dust ensemble by the laser radiation. The smaller the scattering angles are, the lower laser intensities are required. The laser intensity is attenuated by a partially reflecting plate (2) with transmissions between $1 \%$ and $20 \%$.

A Glan-Thompson polarizing prism 32 (3) ensures linear polarization perpendicular to the electrode plane. A quarter-wave plate (4) transforms the linear polarized light into circular polarized light. The fast axis of the quarter-wave plate is rotated by $45^{\circ}$ with respect to the axis of linear polarization of the laser light. This gives equal amplitudes of the parallel and perpendicular wave component.

The laser beam is carefully adjusted by two laser mirrors (5) to be parallel to the electrode.

${ }^{31}$ Helium-Neon laser, head type 1145/P, JDSU

${ }^{32}$ Glan-Thompson polarizing prism, laser grade, type PTOL-10.0-425-675, 425-675nm AR coating, Melles Girot 


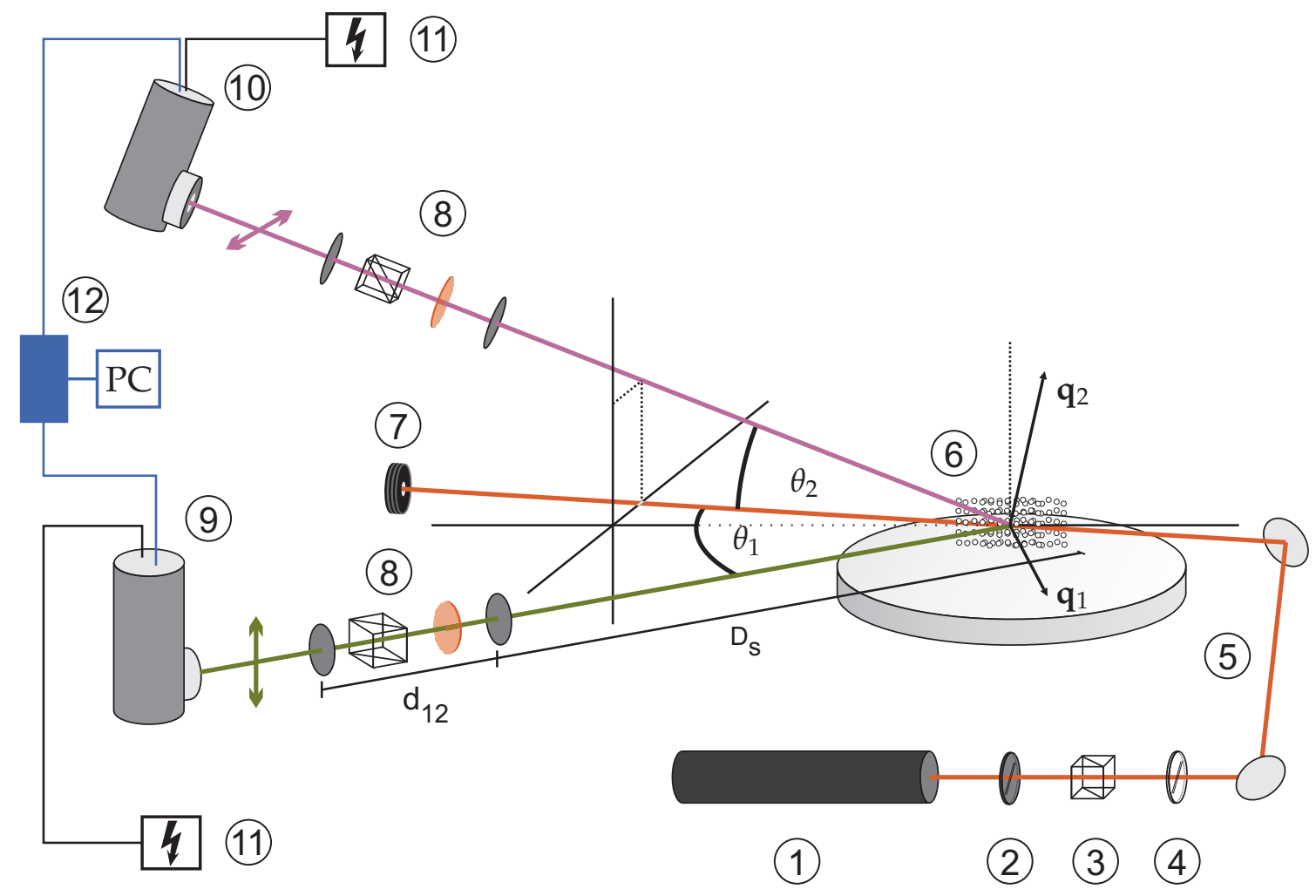

Figure 4.5: Scattering geometry and devices of the DLS experiment: (1) Helium-Neon laser (2) optical attenuator $(1 \%-20 \%)$ (3) Glan-Thompson polarization prism (4) quarter-wave plate (5) laser mirror system (6) dust particle system and discharge chamber (7) beam dump (8) collection system (see text for details) (9) lower photomultiplier (horizontal particle movement) (10) upper photomultiplier (vertical particle movement) (11) stabilized high voltage supply (12) fast data acquisition PCI Express card and personal computer. The quantities $\mathbf{q}_{1(2)}$ and $\theta_{1(2)}$ denote the scattering vector and scattering angle of the lower (upper) light path. $D_{s}$ is the distance between the first aperture of the collection system and dust system and $d_{12}$ is the distance between the apertures.

The second window of the discharge chamber 33 (exit window) is tilted to avoid back reflections into the dust system. Finally, the laser beam is absorbed by an optical beam dump (7).

As discussed in section 2.2.1, only the particle movement parallel to the scattering vector is studied in DLS. Thus, the lower photomultiplier (9) detects the dust movement in the horizontal plane, because $\mathbf{q}_{1}$ is parallel to the electrode. The upper photomultiplier (10) examines the vertical particle motion. As illustrated in the figure 4.5, the scattering vector $\mathbf{q}_{2}$ is not perfectly perpendicular to the electrode, but is tilted by a small angle. As long as the scattering angle $\theta_{2}$ is sufficiently small, the horizontal contribution to $\mathbf{q}_{2}$ can be neglected and the vertical particle movement dominates the intensity fluctuations. This is, of course, a compromise to realize the single laser approach. The exact vertical motion can only be studies with a second laser beam. The scattering angles $\theta_{1}$ and $\theta_{2}$ are below $10^{\circ}$ in the experiments and are determined geometrically in the triangle defined by the paths of incident beam and scattered

${ }^{33}$ For the sake of clarity, the window is not shown in this graph. See figure 4.1 for details. 
light.

The scattered light is detected through collection systems (8) that consist of an entrance aperture $a_{1}$, an interference filter, a Glan-Thompson polarizing prism ${ }^{34}$, and an exit aperture $a_{2}$. The apertures restrict the effective detector area to ensure that only a few coherence areas are detected (see section 2.2.4). If the apertures are chosen too large, the intensity fluctuations are averaged and the amplitude of the autocorrelation function is reduced.

In theory, the degree of coherence is assumed to be perfect, i.e. only a single coherence area is detected. This situation is hardly realized in a real experiment. Hence, the (homodyne) scattering function, as introduced in the theory section 2.3 , has to be adapted to the case of a real experiment. This is done by the introduction of the coherence factor $\beta_{c o h}$ into the scattering function:

$$
F_{\text {hom }}(\mathbf{q}, t)=\langle N\rangle^{2}\left\{1+\beta_{c o h}^{2} F_{S}^{2}(\mathbf{q}, t)\right\},
$$

where $0 \leq \beta_{\text {coh }} \leq 1$. The experimental parameter $\beta_{\text {coh }}$ has to be maximized to improve the contrast the fluctuating signal [86].

The design of the collection system is essential to obtain a $\beta_{\text {coh }}$ close to unity. The best values for the aperture sizes $a_{1}$ and $a_{2}$, distances to the source $D_{s}$, and distances between the apertures $d_{12}$ can be estimated using equation (2.29). After this equation, the best contrast is obtained, if the apertures are minimized. On the other hand, the solid angle defined by the two apertures has to be large enough, to enclose the whole dust particle system, i.e. the distance to the dust system $D_{s}$ has to be sufficiently large. The increase of $D_{s}$ is restricted by the detection limit of the photomultipliers, because the scattered intensity scales like $D_{s}^{-2}$. A compromise has to be found regarding the amplitude of the scattered intensity, the degree of coherence, and the size of the scattering volume.

The best parameters were determined empirically to be $D_{s}=1495 \mathrm{~mm}, d_{12}=320 \mathrm{~mm}$, $a_{1}=2 \mathrm{~mm}$, and $a_{2}=0.8 \mathrm{~mm}$. In the experiments of this work, the cylindrical scattering volume has typically a length of $l=15 \mathrm{~mm}$, defined by the extension of the dust system and a diameter of $d_{l}=0.7 \mathrm{~mm}$. The scattering volume contains a few hundred dust particles. The coherence factor $\beta_{\text {coh }}$ is only of about 0.5 , but this is found to be enough to analyze the decay of the autocorrelation function.

The scattering on perfectly spherical dust grains is only possible within the plane perpendicular to the (linear) polarization of the laser light. Thus, the linear component perpendicular to the electrode is only scattered within the horizontal plane and the other polarization component is only scattered in the vertical plane. Nevertheless, Glan-Thompson prisms are mounted to obtain only the desired polarization. This considers possible contributions due to depolarized dynamic light scattering. In this case, anisotropic particle properties cause a contribution from the horizontal particle movement to the detected signal in the vertical scattering plane and vice versa [13, 14].

The emission from the plasma and the background light is blocked by an interference fil- 
ter 34 with center wavelength of $632.8 \mathrm{~nm}$ and a bandwidth of $1 \mathrm{~nm}$.

High sensitive photomultipliers 35 with a quantum efficiency of about $60 \%$ at $632.8 \mathrm{~nm}$ are used. These detectors have a gain of $9.5 \cdot 10^{6}$ and the dead time is of the order of $25 \mathrm{~ns}$. The voltage divider circuit of the photomultiplier tubes is designed to measure in the photon counting mode as well as in the intensity fluctuation mode. The photon counting mode is relevant if the scattered intensity is low, or if fast measurements are performed as in this work. The photomultiplier delivers a small current peak for each photon that arrives at the photocathode. In the case of high light intensities, the voltage divider circuit has to handle much higher currents. A scheme of the divider network, that is able to manage both cases with sufficient stability, is shown in figure A.1 in the appendix. A $50 \Omega$ load resistor is installed in the voltage divider circuit, that allows fast detection with up to $50 \mathrm{MHz}$.

The divider circuit is connected to a stabilized high voltage supply 36 (11). Voltages between $-1000 \mathrm{~V}$ and $-1200 \mathrm{~V}$ are applied to the divider network.

The photomultiplier signals are detected by a two channel PCI Express transient recorder 37 (12) and saved on the hard drive of a personal computer. No previous amplification of the signal or hardware discrimination of photon peaks is performed. To resolve the fluctuations caused by the rapid dust grain motion over sufficiently long times, the data acquisition card is able to measure with up to $50 \mathrm{MHz}$ with data streaming directly on the computer memory. The size of the data sample is limited by the operating system to $5 \cdot 10^{8}$ points. The fast streaming rate is realized by a low resolution of 8 bit, i.e. the input signal is scaled between 0 and 255. This resolution is still adequate to resolve the photon peaks in the photomultiplier signal.

\subsubsection{Validation of the DLS Setup}

The devices of a DLS setup have to be chosen carefully regarding their contribution of random noise fluctuations to the detected signal:

The laser light source can cause noise due to intensity fluctuations on fast and slow time scales, wavelength instabilities, mode fluctuations, depolarizing effects, and lack of coherence.

All optical components can give rise to unwanted scattered intensity by dust or scratches on the surfaces or back reflection. The vibrations of the setup have impact on the dust ensemble and on the light path relative to the detector.

The photomultiplier contributes to noise by fluctuation of the gain or the supply voltage. Further sources of noise are the dark current, temperature variations of the voltage divider circuit, or bad impedance matching (signal reflection, after pulsing).

\footnotetext{
${ }^{34}$ Interference filter, $\mathrm{CWL}=632.8 \mathrm{~nm} \pm 0.2 \mathrm{~nm}, \mathrm{FWHM}=1 \mathrm{~nm} \pm 0.2 \mathrm{~nm}$, type FL632,8-1, Thorlabs

${ }^{35}$ Photomultiplier tube R3896, Hamamatsu

${ }^{36}$ High voltage supply PM 28B, EMI

${ }^{37}$ PCI Express transient recorder M2i.2020, Spectrum Systementwicklung Microelectronic
} 


\begin{tabular}{cccc}
\hline & $\begin{array}{c}\text { Factorial } \\
\text { Moment }\end{array}$ & Experiment & Theory \\
\hline Coherent & $N^{(1)}$ & 1.000 & 1.000 \\
(unscattered) & $N^{(2)}$ & 1.001 & $1.000 \pm 0.005$ \\
laser light & $N^{(3)}$ & 1.005 & $1.000 \pm 0.013$ \\
& $N^{(4)}$ & 1.017 & $1.000 \pm 0.032$ \\
$4 \cdot 10^{5}$ samples & $N^{(5)}$ & 1.035 & $1.000 \pm 0.086$ \\
& $N^{(6)}$ & 1.066 & $1.000 \pm 0.251$ \\
\hline
\end{tabular}

Table 4.1: Factorial moments of unscattered (coherent) laser light. The photon statistic is calculated for a measurement of $4 \cdot 10^{5}$ samples. The factorial moments are identical within errors with the theoretical predictions for a Poisson distribution.

To ensure, that these unwanted contributions are negligible, the DLS setup is validated by a test that is described by Oliver et al. in [87, p.95]. The unscattered, coherent laser light is detected by the photomultiplier and the signal is analyzed statistically according to the photon events. If contributions from the experiment are absent, the photon statistic should be Poisson. This is evaluated by calculation of the factorial moments $N^{(r)}$ of the photon distribution

$$
N^{(r)}=\frac{<n(n-1) \ldots(n-r+1)>}{<n>^{r}}=1,
$$

where $<>$ denotes the average over $J$ samples, $n$ is the number of photons in a sample, and $r$ denotes the order of the moment.

The result is shown in table 4.1. A number of $2 \cdot 10^{8}$ data points were acquired with $10 \mathrm{MHz}$. The photons are counted in samples with size of $50 \mu \mathrm{s}$ (500 data points). The resulting $4 \cdot 10^{5}$ samples are analyzed according to the photon statistics. The values of the experiment are close to the prediction for a Poisson statistic. The theoretical errors in the table are calculated after [88].

This measurement proves, the components of the setup described above introduce only a negligible distortion to the measurements.

The application of the Siegert relation (2.24) is based on the assumption, that the complex amplitudes of the scattered light show a Gaussian distribution. Thus, only if this assumption holds, the Siegert relation (2.24) can be applied to obtain the self-intermediate scattering function $F_{S}(\mathbf{q}, t)$ in a homodyne DLS experiment (see section 2.2.2).

The distribution of scattered light is analyzed in a similar experiment. This time, a set of $2 \cdot 10^{6}$ samples with lengths of $10 \mu$ s are the basis of the photon statistic.

A perfect Gaussian statistic can only be expected for an experiment with a coherence factor $\beta_{\text {coh }}$ of unity. In a real experiment, the partial coherence leads to an amplitude distribution that shows to be narrower. The Siegert relation can be applied, if the factorial moments of the 


\begin{tabular}{cccc}
\hline & $\begin{array}{c}\text { Factorial } \\
\text { Moment }\end{array}$ & Experiment & Theory \\
\hline Incoherent & $N^{(1)}$ & 1.00 & 1.00 \\
(scattered) & $N^{(2)}$ & 1.35 & $1.35 \pm 0.03$ \\
laser light & $N^{(3)}$ & 2.34 & $2.30 \pm 0.16$ \\
& $N^{(4)}$ & 4.95 & $4.72 \pm 1.07$ \\
$2 \cdot 10^{6}$ samples & $N^{(5)}$ & 12.5 & $11.4 \pm 2.9$ \\
& $N^{(6)}$ & 36.4 & $31.3 \pm 94$ \\
\hline
\end{tabular}

Table 4.2: Factorial moments of scattered (incoherent) laser light. The photon statistic is calculated for a measurement of $2 \cdot 10^{6}$ samples. The factorial moments are in good agreement with the theoretical predictions for the distribution with a coherence factor of $\beta_{c o h}<1$. This measurement justifies the application of the Siegert relation.

distribution are described by [86]

$$
N^{(r)}=\left(1+\beta_{c o h}\right)\left(1+2 \beta_{c o h}\right) \ldots\left(1+(r-1) \beta_{c o h}\right) .
$$

The factor $\beta_{c o h}$ can be obtained from the second factorial moment, $N^{(2)}=\left(1+\beta_{\text {coh }}\right)$. The results of the experiment are shown in table 4.2. The second factorial moment shows, the coherence factor was only $\beta_{c o h}=0.35$ in this experiment. The results from theory and experiment are identical within the errors. This important result justifies the homodyne DLS method to study complex plasmas with micron size dust particles.

\subsubsection{Data Acquisition and Analysis}

The PCI Express card is controlled by a Visual Basic program. The card parameters like acquisition length and frequency can be entered in a graphical user interface. The storage of the data in a text file is managed by the program too. The photomultiplier signals are displayed in a window to control the fluctuation amplitude. The program also allows to manage the pressure and power of the discharge remotely, and the discharge parameters are saved for each data acquisition. Furthermore, the acquisition of the photomultiplier signals for a series of pressures is automated. The user defines a sequence of pressures that has to be examined. The program adjusts the first pressure, waits a certain time to reach a new equilibrium in the dust system and initiates the data acquisition. Then the procedure starts over again and the next pressure is adjusted. This automation simplifies the study of phase transition in the dust system.

The further data treatment is done by C-routines on two multi processor personal computers 38 independently. The first step of the data treatment is to identify and count photon peaks. This is only necessary in the photon mode (fast or low intensity measurements). Each peak

${ }^{38} 1$ ) dual core $2.6 \mathrm{GHz}, 3 \mathrm{~GB}$ RAM; 2) quad core $2.66 \mathrm{GHz}, 3 \mathrm{~GB}$ RAM. 


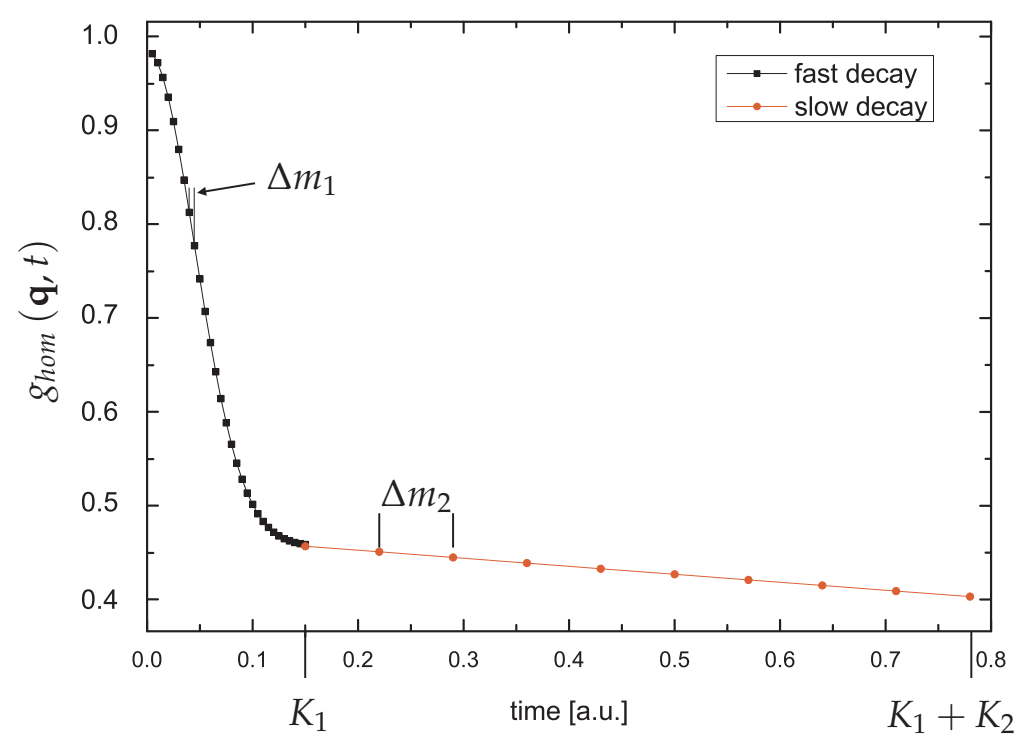

Figure 4.6: An autocorrelation function with a fast decay (squares) and a slow decay (circles). The calculation of the autocorrelation can be adapted to the required time scales by defining a short time window $K_{1}$ with a small increment of the lag time $\Delta m_{1}$ for the fast decay and a large $K_{2}$ and bigger $\Delta m_{2}$ for the slow decay.

with an amplitude above a threshold is counted as a photon. This filters low amplitude noise contributions.

All photons in a predefined time window are added. This can be used to reduce the sample length and temporal resolution, if the acquisition frequency is much faster than the process of interest. In the opposite case, the window size is set to 1 and single photons are regarded.

The next step is to calculate the temporal autocorrelation after 39

$$
\langle n(0) n(m)\rangle=\frac{1}{J} \sum_{j=0}^{J} n_{j} n_{j+m},
$$

where $n_{j}$ denotes the number of photons in the $j$-th sample and $J$ is the number of samples. The sum has to be calculated for all lag times $m$ of interest. To reduce the processing time, the lag time $m$ can be increased in steps $\Delta m$ bigger than 1 . The parameter $\Delta m$ defines the resolution of the autocorrelation function. The second important parameter is $K$ that defines the maximal lag time. This parameter is used to restrict the calculations, because it is not reasonable to calculate the autocorrelation function for lag times much larger than the decay time of the fluctuations.

In a more refined procedure, three different maximal lag times can be defined with $K_{1}<$ $K_{2}<K_{3}$. For each $K$, the lag time can be increased independently, i.e. $\Delta m_{1} \neq \Delta m_{2} \neq \Delta m_{3}$. This allows to study the fluctuating signals on different time scales with different resolutions. For example, a signal fluctuates on a fast and a slow time scale. The autocorrelation function shows two separated decays in this case, as demonstrated in figure 4.6. The fast decay of the autocorrelation can be analyzed with the first window $K_{1}$ with high temporal resolution $\Delta m_{1} \approx 1$, and the slow decay can be analyzed within the same calculation with a second

${ }^{39}$ See section 2.1.1 
window $K_{2}$ and lower temporal resolution $\Delta m_{2}>1$.

The final step is to normalize the autocorrelation function. The autocorrelation function calculated so far is $\langle n(0) n(t)\rangle$. As introduced in section 2.2.2, this is proportional to $I_{\text {hom }}(\mathbf{q}, t)$, and therefore to $F_{\text {hom }}(\mathbf{q}, t)$. The self-intermediate scattering function $F_{S}(\mathbf{q}, t)$, which contains all dynamical information of the system, can be obtained by normalization:

$$
g_{\text {hom }}(\mathbf{q}, t):=\frac{\langle n(0) n(t)\rangle}{\langle n\rangle^{2}}=\frac{I_{h o m}(\mathbf{q}, t)}{\langle I\rangle^{2}}=\frac{F_{h o m}(\mathbf{q}, t)}{\langle N\rangle^{2}}=1+\beta_{c o h}^{2} F_{S}^{2}(\mathbf{q}, t),
$$

where $\langle n\rangle$ is the average number of detected photons, $\langle I\rangle$ is the average intensity of the scattered light, and $\langle N\rangle$ is the average number of scatterers (dust particles 40 .

The autocorrelation $g_{\text {hom }}(\mathbf{q}, t)$ is the result of the measurement and $F_{S}(\mathbf{q}, t)$ is connected to the dynamical variables as described in section 2.3 .

The calculation of $g_{\text {hom }}(\mathbf{q}, t)$ with the $2.6 \mathrm{MHz}$ processor takes 90 minutes for a number of $J=10^{6}$ samples, a maximal lag time of $K=10^{5}$, and a resolution of $\Delta m=1$. The multi-core architecture allows the calculation of four autocorrelation functions at the same time.

Finally, the autocorrelation function is fitted with a Gaussian function, an exponential decay or a combination of both, depending on the present particle motion. The fitting is performed by routines of the NAG-library 41 implemented in another C-routine. This procedures make it feasible to fit a huge set of autocorrelation functions automatically. For all extended experiments or measurements with high temporal resolution, the large number of autocorrelation functions can only be coped by the aid of the automated fitting routine. The accuracy of the fits is estimated by the $\chi^{2}$-parameter delivered by the procedure. Additionally, an evaluation by the user is possible by plotting the original data and the fit. The automatic fitting routine is a very important tool for the experiments presented in this work.

\footnotetext{
${ }^{40}$ Here, the Siegert relation is applied: $F_{h o m}(\mathbf{q}, t)=\langle N\rangle^{2}+\langle N\rangle^{2} \beta_{c o h}^{2} F_{S}^{2}(\mathbf{q}, t)$. ${ }^{41}$ http://www.nag.com/numeric/numerical_libraries.asp
} 



\section{Chapter 5}

\section{Results}

This chapter presents the application of DLS on complex plasmas with focus on two main branches of experiments. Firstly, the ability of DLS to measure in a strongly coupled system of scatterers is examined. Here, the question of the kind of dynamical parameters available by DLS is answered. Secondly, the DLS is used to study the dynamics of the dust component in a complex plasma under gravity with emphasis on phase transitions.

This chapter is ordered as follows. Section 5.1 introduces the form of autocorrelation function that is observed in the DLS experiments on phase transitions. Small deviations from a pure decay form at low scattering angles are discussed. In section 5.2, the errors to be expected for the DLS measurement are studied. These two sections demonstrate the applicability of DLS on complex plasmas.

Section 5.3 examines phase transitions of the dust component induced by variation of the discharge pressure or power. The impact of various experimental parameters on the phase transition is studied. In section 5.4 the hysteresis loop as predicted by theory (see section 3.3.2) is examined. The section 5.5 answers the question, if the diffusive motion of the scatterers on length scales comparable to the inter-particle separation can be studied by DLS even in a strongly coupled complex plasma.

\subsection{The Ballistic Regime and Benchmark of DLS with the CCD Technique}

One of the most fundamental problems in a DLS experiment is to find an adequate model to describe the decay of the autocorrelation function. In previous DLS experiments on dusty plasmas, a Gaussian decay [17] or an exponential decay was observed as in [89, 18]. But these experiments were done with a nano-scaled dust component with relative weak coupling between the dust particles. The particles studied in this work have diameters of several microns. The dust particle charge is much higher and the interactions between the particles and between particles and the electric field in the sheath of the plasma is much stronger. The form of the autocorrelation function for strongly coupled complex plasmas is therefore still an open question that will be answered at the beginning of this section. A broad range of scattering 
angles was examined for the horizontal and the vertical component of motion. Some kind of particle motions contribute disturbances and noise to the autocorrelation function. This will be discussed briefly. Finally, the observations done with DLS will be compared to results of the well-established CCD technique.

A DLS experiment was performed on a complex plasma containing $3 \mu \mathrm{m}$ MF-particles 42 in an ordered phase state at a discharge pressure of $20 \mathrm{~Pa}$ and a discharge power of $2 \mathrm{~W}\left(40 \mathrm{~V}_{\mathrm{PP}}\right)$. The scattered light was detected in homodyne mode with a scattering angle of $9^{\circ}$. The sample time was $20 \mathrm{~s}$ with an acquisition frequency of $10 \mathrm{MHz}$.

The normalized autocorrelation function $g_{\text {hom }}(\mathbf{q}, t)$ obtained in this measurement is shown in figure 5.1 and explicitly reveals a Gaussian decay form. The residuals corresponding to the Gaussian fit are below $0.3 \%$. That means, ballistic particle motion is observed, even though the strong coupling between the scatterers is present.

This might be a surprising result on the first glance. But, as discussed in section 2.2.3, it is a question of the characteristic length scale of the DLS measurement. This length scale is defined by the inverse absolute value of the scattering vector $q^{-1}$. For small length scales compared to the mean free path of dust-dust collisions, the particle movement is free. For the parameters of this measurement, the inverse scattering vector was $q^{-1}=0.6 \mu \mathrm{m}$. This is small compared to the average particle distance of about $200 \mu \mathrm{m}$.

As described in section 2.3, the average velocity-squared in one dimension can be acquired from the Gaussian decay time by

$$
\left\langle v_{x}^{2}\right\rangle=\frac{2}{\omega^{2} q^{2}}=\frac{1}{2 \sigma^{2} q^{2}}
$$

where $q$ and $\omega=2 \sigma$ denote the scattering vector and the width of the Gaussian function.

The introduction of a particle temperature $T_{d}$ is justified, because one condition in the derivation of the Gaussian decay form in section 2.3.2 is a Maxwell-Boltzmann velocity distribution. The thermal energy is defined by $T_{e V}:=k_{B} T_{d}$, where $k_{B}$ is the Boltzmann constant.

The decay time, given as the half width of the Gaussian $\sigma=0.5 \omega$, was measured as $0.9 \mathrm{~ms}$, so the average velocity was $504 \mu \mathrm{m} / \mathrm{s}$. Hence, the decay times for the density fluctuations in a complex plasma are expected to be of the order of $1 \mathrm{~ms}$ in the ordered state and even faster for the melted state 43 . This is about two orders of magnitude faster than what is seen in most DLS experiments on biological or chemical systems of scatterers in solutions [13].

To prove the assumption of ballistic particle motion for different scattering angles, a test suggested by Nossal et al. in [21] was applied. The self-intermediate scattering function for

\footnotetext{
${ }^{42}$ Melamine-Formaldehyde-particles

${ }^{43}$ Note, for small angle scattering experiments, the decay time can be larger, considering that $\tau \propto q^{-1}$.
} 
Figure 5.1: The normalized autocorrelation function $g_{\text {hom }}(\mathbf{q}, t)$ measured in the ordered state of a complex plasma. The scattering angle was $9^{\circ}$ and the data acquisition lasted $20 \mathrm{~s}$. A Gaussian fit describes the data with high goodness. The residuals are below $0.3 \%$ as shown in the lower figure. A Gaussian decay form represents ballistic or free particle motion. The corresponding decay time is $\sigma=0.9 \mathrm{~ms}$.

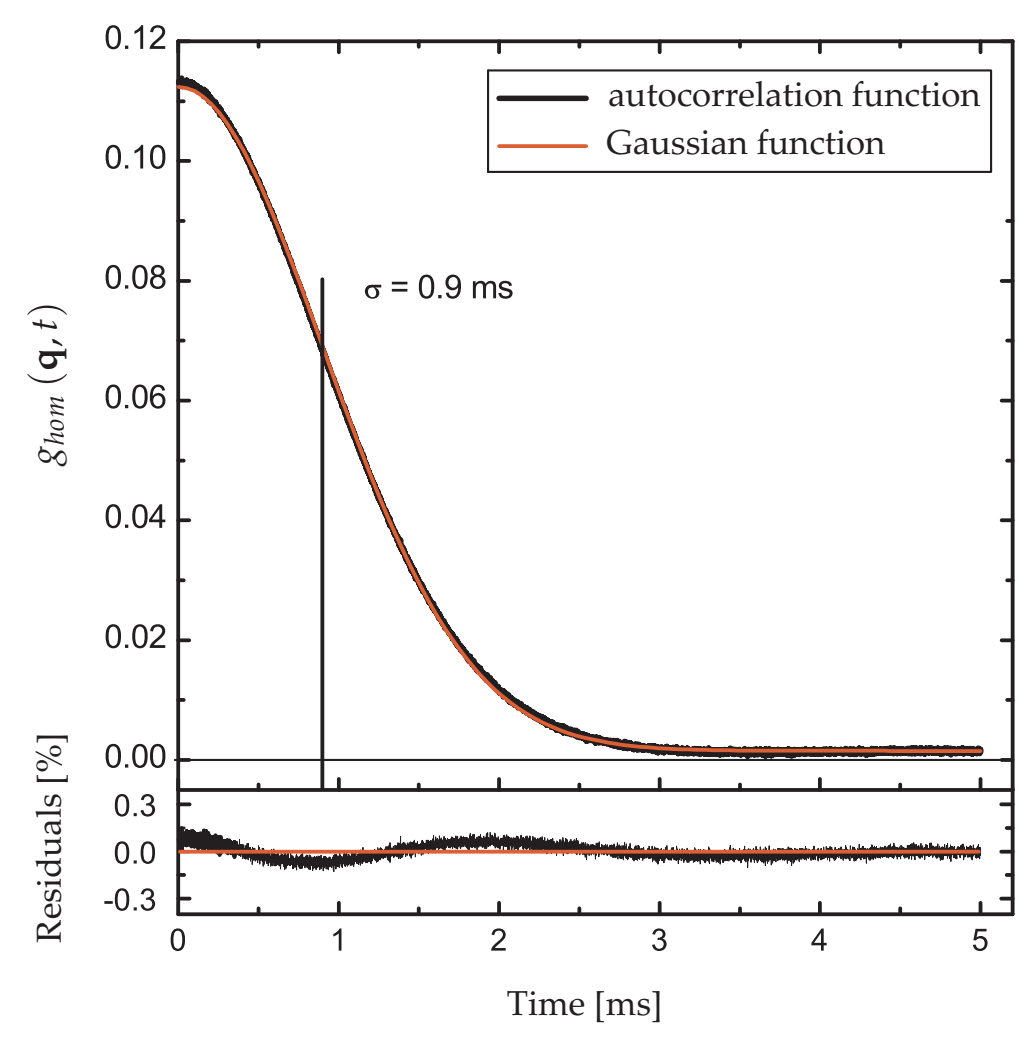

ballistic scatterers was introduced in section 2.3.2 and is repeated here:

$$
F_{S}(\mathbf{q}, t)=\exp \left(-\frac{1}{2} q^{2}\left\langle v_{z}^{2}\right\rangle t^{2}\right)
$$

According to this equation, $F_{S}(\mathbf{q}, t)$ should be a function of $Z=q t$ in the ballistic regime, i.e. a function of the scattering angle $\theta$ and time $t$. If $F_{S}(\mathbf{q}, t)$ is measured for different scattering angles and plotted against $Z$, all curves should superimpose in the case of ballistic motion.

Figure 5.2 (a) illustrates a measurement of $F_{S}(\mathbf{q}, t)$ versus time for scattering angles between $1^{\circ}$ and $9^{\circ}$. The time-axis is scaled logarithmic for the sake of better distinctness of the data. The particle system was in an ordered state at $20 \mathrm{~Pa}$, and the horizontal component of particle motion was detected. The decay time varies from $2 \mathrm{~ms}$ to $20 \mathrm{~ms}$, as the scattering angle changes from $9^{\circ}$ to $1^{\circ}$.

In (b), the same data are plotted against $Z=q t$, and as can be seen, the scattering functions $F_{S}(Z)$ lie on top of each other with good accuracy. Small deviations are due to the error in determination of the scattering angle. The most important sources of errors in a DLS experiment will be discussed in the next section.

A similar experiment was done at a discharge pressure of 2 Pa beyond the melting transition. The dust particle system shows no order anymore and the dust particle energy reaches up to $100 \mathrm{eV}$ in the horizontal plane. The related decay times are $2 \cdot 10^{-5} \mathrm{~s}$ for a scattering angle of $9^{\circ}$ and $1 \cdot 10^{-4} \mathrm{~s}$ at $1^{\circ}$. The self-intermediate scattering functions plotted as a function 
(a)

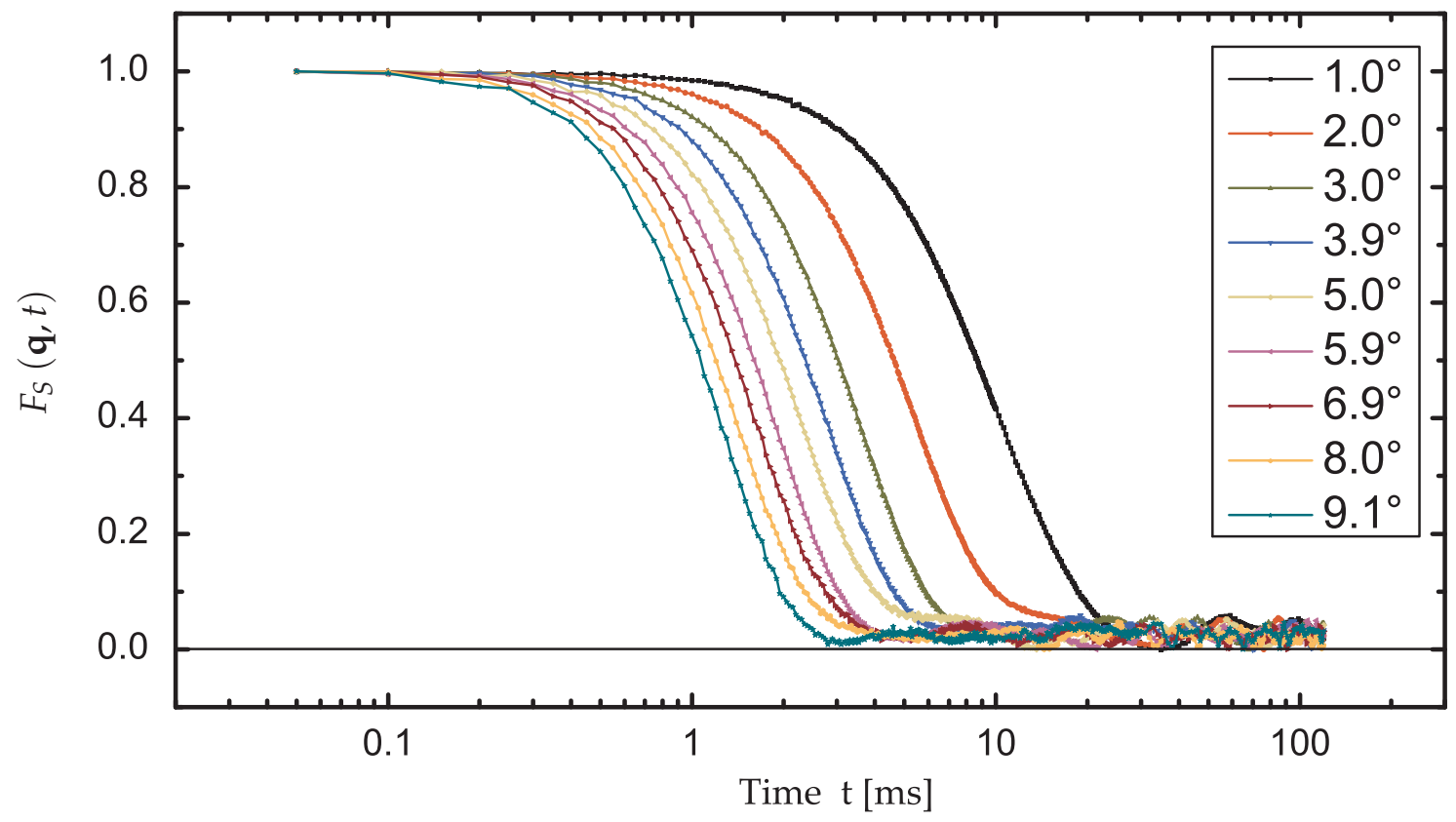

(b)

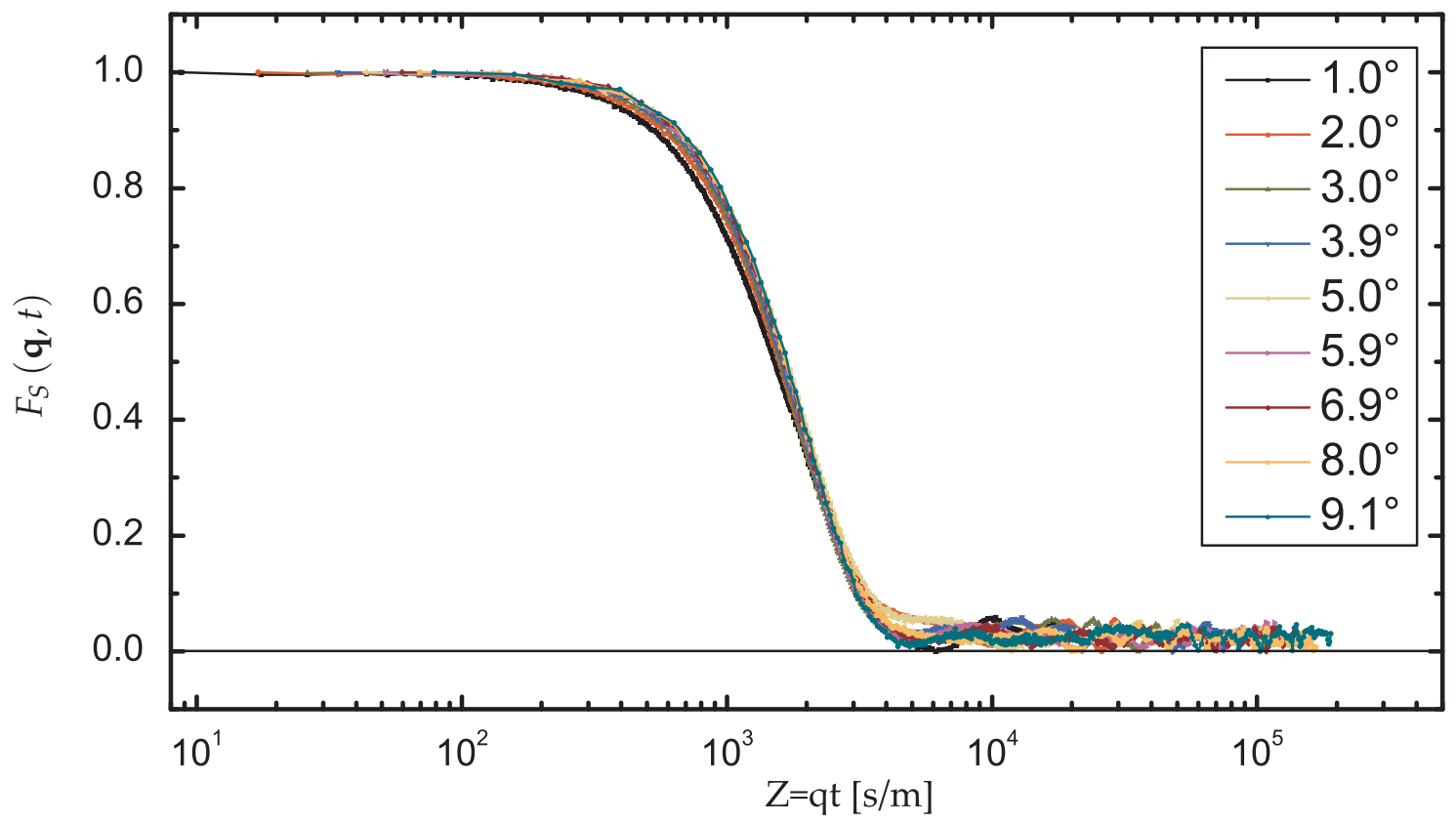

Figure 5.2: Proof of ballistic particle motion for scattering angles between $1^{\circ}$ and $9^{\circ}$ in the ordered phase state at a discharge pressure of $20 \mathrm{~Pa}$. (a) The self-intermediate scattering functions $F_{S}(\mathbf{q}, t)$ at different scattering angles for particle movement in the horizontal plane. The decay time rises from 2 $\mathrm{ms}$ to $20 \mathrm{~ms}$ while the scattering angle is decreased. (b) The scattering functions $F_{S}(\mathbf{q}, t)$ superimpose if they are plotted over $Z=q t$, which proves ballistic particle motion. Small deviations appear due to the measurement error of the scattering angle $\theta$. 
Figure 5.3: Self-intermediate scattering function $F_{S}(\mathbf{q}, t)$ for a scattering angle below $4^{\circ}$ in a state close to the phase transition. Next to the Gaussian decay an additional contribution appears. A combination of Gaussian and exponential function fits more precisely than a pure Gaussian function. The decay time changes from $\sigma_{G}=0.11 \mathrm{~s}$ for the Gaussian fit to $\sigma_{G E x p}=0.09 \mathrm{~s}$ for the combined fit.

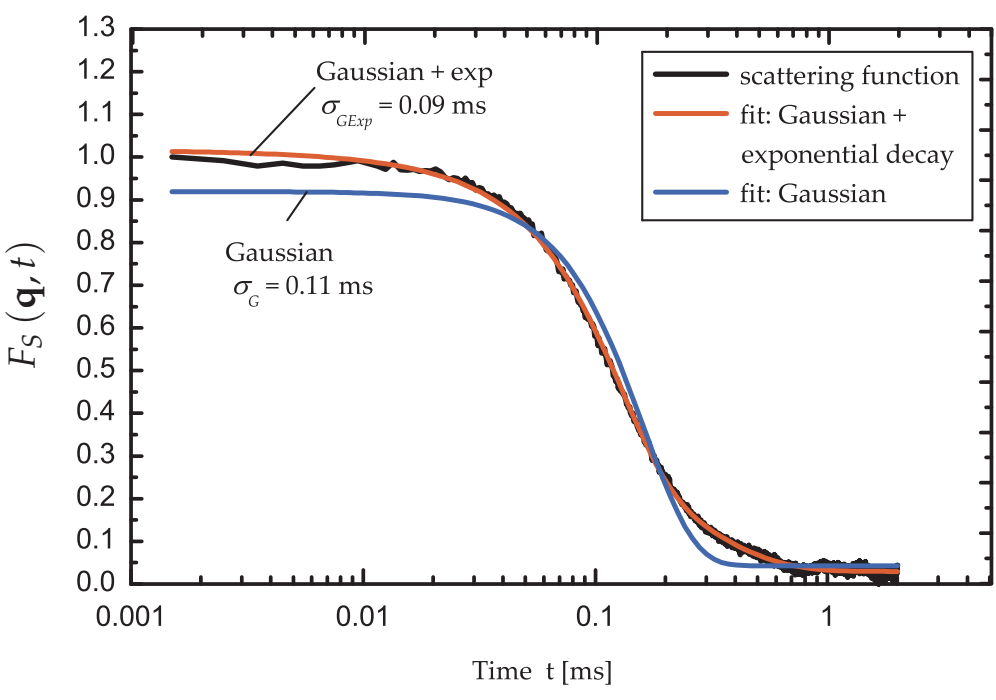

of $Z$ also superimpose in this case, within the measurement error of the scattering angle $\theta$. The plot similar to figure 5.2 for this case can be found in the appendix, figure A.2

DLS measurements in the vertical direction 44 also reveal autocorrelation functions with Gaussian decay forms. That means, neither the electric field in the plasma sheath in the direction of the movement, nor the large kinetic particle energy and related higher collision rates, affects the kind of motion on the length scale of the DLS measurement $q^{-1}$.

DLS experiments with scattering angles from $4^{\circ}$ up to backward scattering at $175^{\circ}$ were seen to take place in the ballistic regime as well. The choice of the scattering angle is a question of the intensity of the scattered light and the data acquisition rate of the hardware. Backward scattering at $175^{\circ}$ allows examinations on very fast time scales, because the length scale $q^{-1}$ is of the order of $0.05 \mu \mathrm{m}$. This can be used to study the particle dynamics with high temporal resolution of about $1 \mathrm{kHz}$. Note, only the horizontal movement can be studied in the backscattering mode.

Small deviations from a pure Gaussian decay are noticed at scattering angles below $4^{\circ}$, if the system is close to the phase transition. Here, a second contribution to the autocorrelation function appears as shown in figure 5.3. The x-axis is scaled logarithmic again to see the differences between data and fit more easily. The pure Gaussian function cannot fit the data with high precision. The Gaussian fit delivers a decay time of $\sigma_{G}=0.11 \mathrm{~ms}$. A fit with a combination of Gaussian function and exponential decay gives better results. The decay time for the combined fit is $\sigma_{\text {GExp }}=0.09 \mathrm{~ms}$. Hence, even in these cases, the Gaussian decay time $\sigma$ can be acquired, but it is recommended to measure at larger scattering angles to reach the pure ballistic regime. The exponential contribution describes diffusive motion of the particles. The DLS in the diffusive regime of particle movement will be discussed in more detail in section 5.5 .

\footnotetext{
${ }^{44}$ Here, the scattering vector $\mathbf{q}$ is nearly perpendicular to the electrodes (see experimental section 4.3.1.
} 
There are some other effects that can influence the autocorrelation function, but remain of minor importance for most cases. One example is given by fluctuations of the autocorrelation function around the baseline, as can be seen in figure 5.2. They are from number fluctuations, dust density fluctuations on fast time scales or streaming dust particles. These fluctuations are only important for short time sampling, where they can lead to large statistical errors. The contributions to the error of a DLS experiment will be discussed in the next section in more detail.

The presence of dust acoustic waves (DAW) does not influence the determination of the correct decay time of the autocorrelation function, because the time scales are separated. The dust acoustic wave frequency is less than $100 \mathrm{~Hz}$, and so the time scale is at least 10 times slower than the Gaussian decay. On the time scales of interest, the DAW are only noticeable by a higher baseline of the autocorrelation function.

In the final part of this section, a benchmark of the DLS method in the ballistic regime by simultaneous CCD observations is presented. The CCD technique allows the identification and tracking of single particles and gives information about the dynamics of the system that can be compared to the DLS measurements directly. This is not possible in common DLS experiments on nano particles.

In this experiment the movement in the horizontal plane is studied. The number of particles probed are about 700 for DLS and about 900 for the CCD measurement. Note, not the very same particles were analyzed because the shape of the detected regions are different. The DLS scattering volume had a cylindrical shape with a length of $l=15 \mathrm{~mm}$ and a diameter of $d_{l}=0.7 \mathrm{~mm}$, whereas the CCD method attained information from a plane with dimension of $10 \cdot 12 \mathrm{~mm}^{2}$. The average particle distance in the horizontal plane was of the order of $400 \mu \mathrm{m}$.

The particle motion in the horizontal plane is expected to be isotropic, but this cannot be assumed for the vertical direction. The vertical extension of the DLS scattering volume, albeit small, might introduce some error due to the anisotropy.

The assumption of isotropy in the horizontal plane was justified by earlier CCD camera observations. Hence, the DLS velocities can be extended to two-dimensional velocities, and the related thermal energies can be compared to the CCD results. This experiment is illustrated in figure 5.4. The discharge pressure was decreased from $55 \mathrm{~Pa}$ to $1 \mathrm{~Pa}$ at a constant discharge power of $4 \mathrm{~W}$, which forced a melting transition in the dust particle system. The measurement was done in Argon using $3 \mu \mathrm{m}$ MF-particles. The two-dimensional thermal energy of the particles is shown for the DLS (squares) and CCD technique (circles).

At low pressures and fast particle movement, the CCD camera method fails to determine the particle velocity, and a saturation of the thermal energy is seen. The CCD approach is limited to the ordered phase state, due to the low frame rate of the camera. This is discussed in detail in section 4.2.2. In the experiment here, a frame rate of $52 \mathrm{~s}^{-1}$ is used, which is sufficient to resolve the motion in the strongly coupled state above $22 \mathrm{~Pa}$.

The thermal energies measured by DLS in this pressure range are much higher and indi- 
(a)

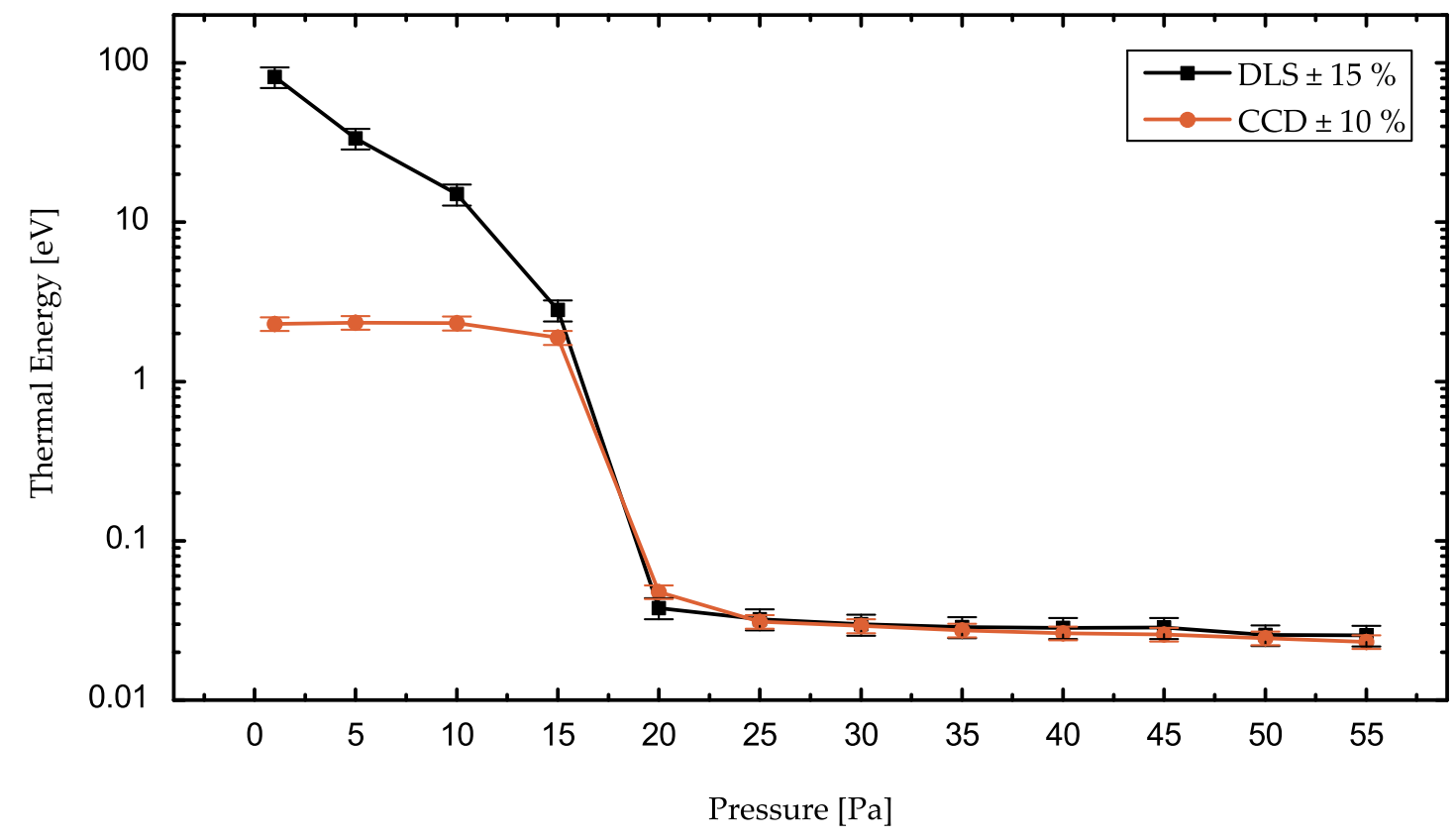

(b)

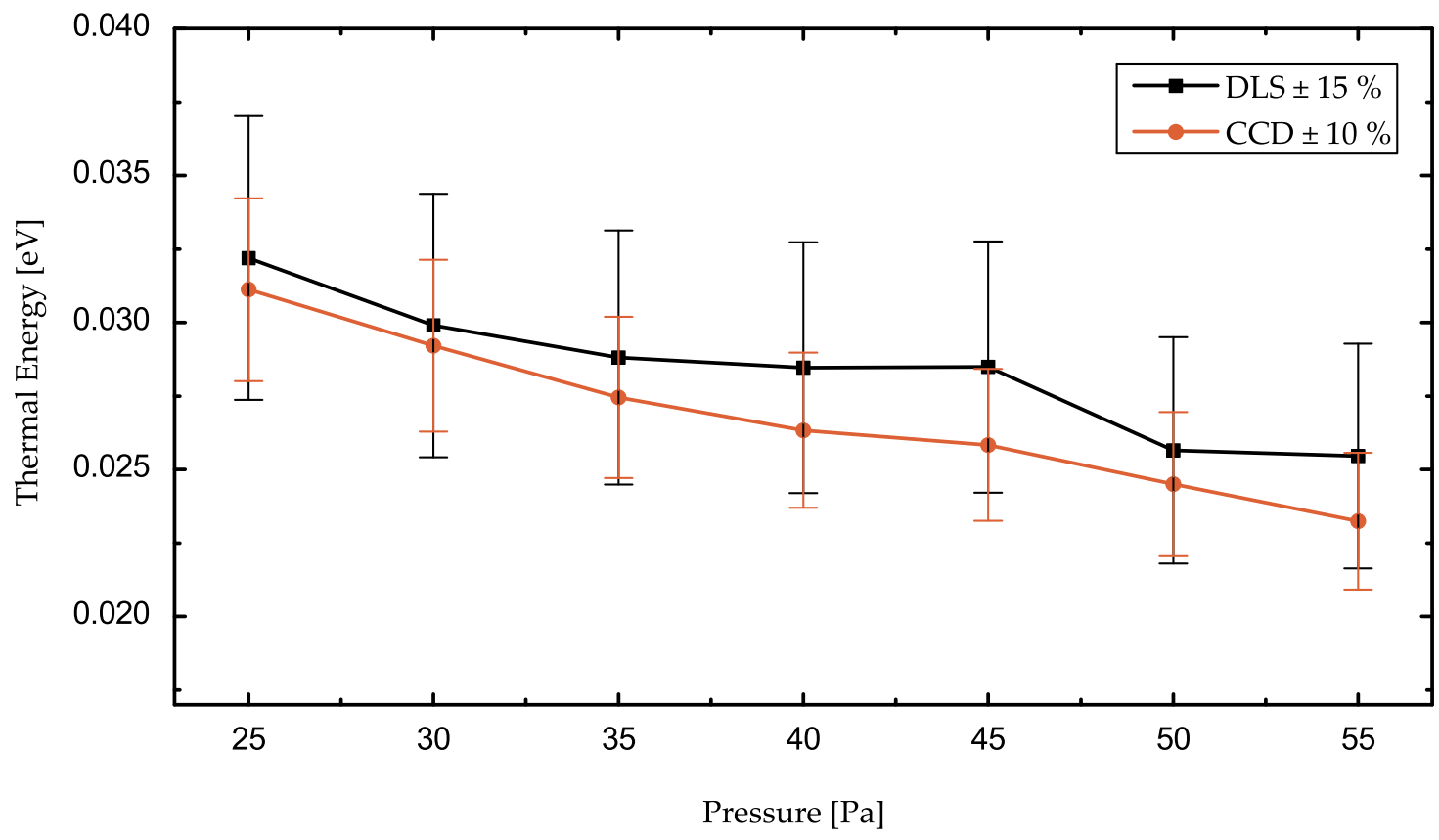

Figure 5.4: Benchmark of the DLS method (squares) by simultaneous CCD observation (circles). In (a), the curve of the CCD measurement saturates in the melted state at low pressures. The determination of the correct velocities fails due to wrong particle tracking. The DLS measurement shows an exponential increase of the thermal energy at decreasing pressure. (b) A very good agreement is found in the ordered state that justifies the DLS approach. Both curves decline in a similar way and the data points are close together with overlapping error bars. 
cate an exponential growth for a decrease of pressure. The fast particle movement causes no principal problems for a DLS experiment. As discussed at the beginning of this section, even at $2 \mathrm{~Pa}$ the autocorrelation is Gaussian, and thus, even in the melted state a Maxwell-Boltzmann velocity distribution is observed. As shown in the next section, the error of the DLS measurement is even lower for faster motions.

At pressures above $20 \mathrm{~Pa}$, the particles arrange to an ordered structure. In this case, good agreement between DLS and CCD measurements is found as shown in figure 5.4 (b). Both curves show a similar decrease and the data points are close together with overlapping error bars. This also justifies the assumption of an isotropic system in the horizontal plane. The errors for the DLS approach used here are treated in following section 5.2. For the measurement shown, the error of DLS is $\pm 15 \%$. The error for the CCD observation is due to wrong particle identification and bad tracking of the particles through the image sequence and is assumed to be $\pm 10 \%$.

In summary, the results presented in this section reveal ballistic particle motion for all pressures relevant to study phase transitions in a complex plasma. The length scale of the DLS measurement $q^{-1}$ is appropriate to study free particle motion in a large range of scattering angles between $9^{\circ}$ and $175^{\circ}$. The applicability of DLS on complex plasmas was proven by explicit measurements at angles between $1^{\circ}$ and $9^{\circ}$.

The electric field in the sheath of the plasma does not affect the type of motion on the specific length scales. The decay form for DLS in the vertical direction is Gaussian as well. Only the time scales are faster by a factor of about 10. The two component setup as described in 4.3.1 can be realized to perform experiments in the ballistic regime.

The final benchmark of the method is done by aid of standard video observation. The agreement between both methods is excellent in the ordered state. This proves the assumptions made in the section on theory and setup of DLS on the kinetic level of single particle movement. Such a benchmark is not possible for common DLS experiments on scatterers in solutions [13].

DLS offers a unique technique to study the highly energetic state of a complex plasma by examining the thermal energy. This parameter can be used as the characteristic quantity to analyze phase transitions in detail. This will be done in the section 5.3 .

\subsection{Estimation of the Error of DLS Measurements}

In this section, the sources of error relevant in a DLS experiment are discussed. The errors are estimated by statistical methods and Gaussian error propagation.

There are three dominant sources of errors in the determination of the thermal energy of dust particles $T_{e V}$ by means of DLS. Firstly, the limited number of dust particles in the scattering volume leads to a statistical fluctuation of the measured thermal energy. This can be 


\begin{tabular}{ccccccc}
\hline & \multicolumn{2}{c}{$4^{\circ}$} & \multicolumn{2}{c}{$9^{\circ}$} & \multicolumn{2}{c}{$175^{\circ}$} \\
$T_{D A Q}$ & $\begin{array}{c}\text { Relative } \\
\text { Error of } \sigma \\
{[\%]}\end{array}$ & $\chi^{2}$ & $\begin{array}{c}\text { Relative } \\
\text { Error of } \sigma \\
{[\%]}\end{array}$ & $\chi^{2}$ & $\begin{array}{c}\text { Relative } \\
\text { Error of } \sigma \\
{\left[10^{-6}\right]}\end{array}$ & $\begin{array}{c}\chi^{2} \\
{[s]}\end{array}$ \\
\hline 0.001 & - & - & - & - & 32.2 & 107 \\
0.01 & - & - & - & - & 33.0 & 107 \\
0.2 & 17.3 & 80 & 13.8 & 19.2 & 7.6 & 11 \\
0.5 & 11.5 & 31 & 8.7 & 7.00 & 3.7 & 2.1 \\
1.0 & 7.7 & 16 & 6.4 & 3.72 & 2.7 & 1.0 \\
2 & 6.2 & 9.2 & 4.7 & 2.13 & 2.2 & 0.5 \\
5 & 3.6 & 4.5 & 3.1 & 1.00 & 1.5 & 0.2 \\
10 & 1.7 & 3.5 & 1.5 & 0.43 & 1.2 & 0.1 \\
20 & 0.8 & 3.3 & 0.8 & 0.34 & 0.6 & 0.06 \\
\hline
\end{tabular}

Table 5.1: Relative statistical errors of the decay time $\sigma$ for different acquisition times $T_{D A Q}$. The average $\chi^{2}$ value describes the goodness of the Gaussian fit analysis. The results for scattering angles of $4^{\circ}, 9^{\circ}$, and $175^{\circ}$ are shown.

countered by a longer data acquisition time $T_{D A Q}$ to improve the temporal average. This error contribution is described by statistics of the decay time $\sigma$ as obtained from the Gaussian fit of the autocorrelation function. The standard deviation of $\sigma$ is determined for different acquisition times $T_{D A Q}$. This analysis were repeated for three scattering angles $\theta$.

Furthermore, the calculation of the thermal energy requires the measurement of experimental parameters. This leads to systematic errors. The second and third major source of error are therefore given by the error in the determination of the scattering angle $\theta$ and the particle radius $a$. These contributions are considered in a Gaussian propagation of error calculation as described later.

To examine the statistical fluctuations of $T_{e V}$, a long measurement was divided into parts with equal length. The length of the samples were varied between $20 \mathrm{~s}$, to examine the error of long acquisition times, and $1 \mathrm{~ms}$, to get the error of short measurements. The average and standard deviation of the Gaussian decay time $\sigma$ were calculated for a set of the data samples of same length. The experiment was done for scattering angles of $4^{\circ}, 9^{\circ}$, and $175^{\circ}$. The particle system was in the ordered phase state at a discharge pressure of $40 \mathrm{~Pa}$ for small angles and $20 \mathrm{~Pa}$ at backward scattering.

The results of the statistics are shown in table 5.1. The relative error of $\sigma$ is the ratio of standard deviation and average of $\sigma$ given in percentage. The $\chi^{2}$-fit parameter describes the goodness of the Gaussian fit. For scattering angles of $4^{\circ}$ and $9^{\circ}$, the decay of the autocorrelation function could not be resolved for acquisition times below $0.01 \mathrm{~s}$. The relative errors are about $1 \%$ for $20 \mathrm{~s}$ of acquisition and $20 \%$ for short measurements of $0.2 \mathrm{~s}$. To get a high temporal resolution, backward scattering can be used. At $175^{\circ}$, a relative error of about $30 \%$ 


\begin{tabular}{ccccccc}
\hline & \multicolumn{2}{c}{$4^{\circ}$} & \multicolumn{2}{c}{$9^{\circ}$} & \multicolumn{2}{c}{$175^{\circ}$} \\
$T_{D A Q}$ & \multicolumn{2}{c}{ Relative Error } & \multicolumn{2}{c}{ Relative Error } & \multicolumn{2}{c}{ Relative Error } \\
& $v_{x}$ & $T_{e V}$ & $v_{x}$ & $T_{e V}$ & $v_{x}$ & $T_{e V}$ \\
{$[s]$} & {$[\%]$} & {$[\%]$} & {$[\%]$} & {$[\%]$} & {$[\%]$} & {$[\%]$} \\
\hline 0.001 & - & - & - & - & 47.15 & 67.2 \\
0.01 & - & - & - & - & 48.99 & 69.8 \\
0.2 & 25.3 & 37.5 & 20.1 & 29.9 & 10.6 & 17.2 \\
0.5 & 17.2 & 26.9 & 12.5 & 19.9 & 5.2 & 11.1 \\
1 & 12.2 & 20.7 & 9.5 & 16.2 & 3.8 & 9.9 \\
2 & 10.3 & 18.5 & 6.6 & 13.0 & 3.1 & 9.5 \\
5 & 7.4 & 15.5 & 5.2 & 11.6 & 2.1 & 8.9 \\
10 & 6.0 & 14.2 & 2.8 & 9.9 & 1.7 & 8.7 \\
20 & 5.5 & 13.8 & 2.8 & 9.9 & 0.8 & 8.5 \\
\hline
\end{tabular}

Table 5.2: Relative error of the one-dimensional particle velocity $v_{x}$ and the corresponding thermal energy $T_{e V}$ for different acquisition times $T_{D A Q}$. The errors are obtained by Gaussian propagation of error calculations on the basis of the data for $\sigma$ from table 5.1. The errors for scattering angles of $4^{\circ}, 9^{\circ}$, and $175^{\circ}$ are presented.

has to be expected for an acquisition time of $1 \mathrm{~ms}$.

The $\chi^{2}$-parameter exhibits a less confident fitting for shorter acquisition times. Considering that the autocorrelation function is an average itself, a shorter acquisition time results in a more noisy autocorrelation function. This leads to a larger standard deviation of $\sigma$.

The data in table 5.1 indicate that the ratio $T_{D A Q} / \sigma$ has to be sufficiently large to obtain a small relative error. Note, the decay time $\sigma$ drops as the scattering angle is increased, because $\sigma \propto q^{-1}=\lambda /(4 \pi \sin \theta / 2)$. The average decay times of the measurements were $2.2 \mathrm{~ms}$ at $4^{\circ}$, $0.9 \mathrm{~ms}$ at $9^{\circ}$ and $0.08 \mathrm{~ms}$ at $175^{\circ}$.

The decay time $\sigma$ is smaller in the melted state, and therefore a smaller statistical error for the same acquisition time can be assumed. But, another contribution to the error appears that is only important for highly disordered state. The presence of dust acoustic waves can lead to larger statistical errors, in particular when the acquisition time is shorter that the oscillation time of the wave. The frequency of dust acoustic waves are typically between 10 $\mathrm{Hz}$ and $100 \mathrm{~Hz}$. Therefore, the acquisition time has to be of the order of a second, to reduce the contribution of the dust acoustic waves to the error. Thus, the errors in table 5.1 can be used as an upper limit for the experiments in this work, as long as the acquisition time is larger than a second.

Next to the statistical fluctuations due to the finite size of the particle ensemble, systematic errors have to be considered. The systematic error in the calculation of the one-dimensional average velocity $\sqrt{\left\langle v_{x}^{2}\right\rangle}=1 /(\sqrt{2} \sigma q)$ is mainly caused by the measurement error of the scattering angle $\theta$ in $q=4 \pi / \lambda \sin (\theta / 2)$. The scattering angle was determined geometrically by measuring two sides of the triangle defined by the incident laser beam, the light path of the 
Figure 5.5: An autocorrelation function that exhibits fluctuations due to streaming particles. The Gaussian fit does not decay exactly to zero due to the fluctuations. This leads to a small error in the determination of the decay time $\sigma$. For reasons of illustration, the peak of the autocorrelation function is cropped at 0.1 . The peak of the correlation function is at 0.56 .

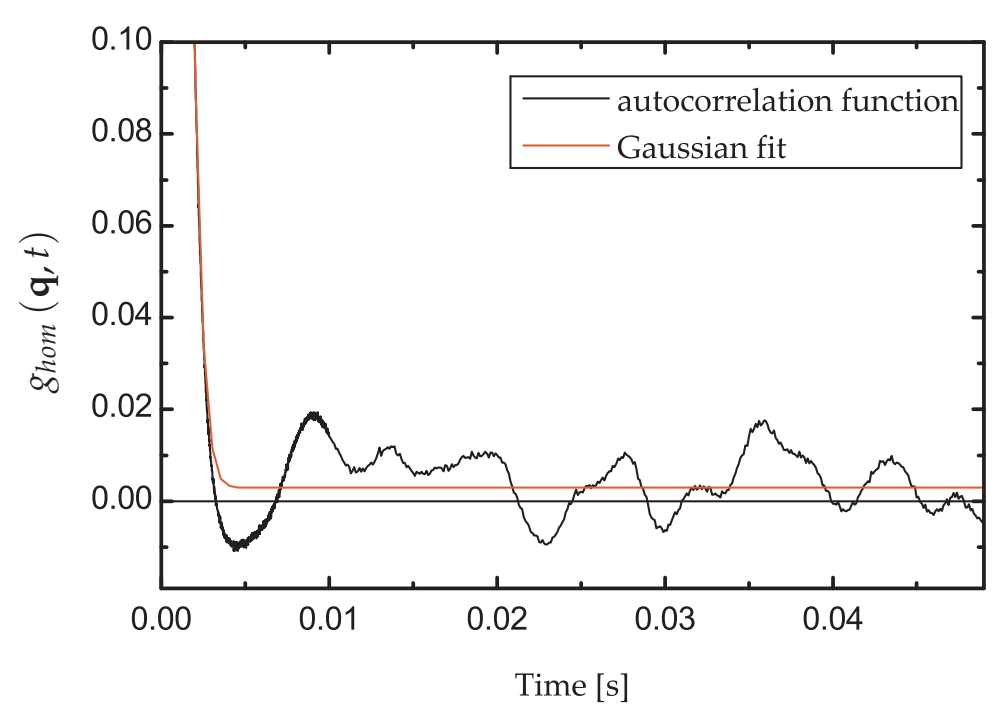

scattered light and the position of the first aperture. The error in measuring the length of the triangle sides are assumed to be less than $\pm 5 \mathrm{~mm}$. The formalism of Gaussian propagation of error is used to obtain the error of the scattering angle $\delta \theta$ leading to the error of the scattering vector $\delta q$ that finally gives the error of the particle velocity $\delta v$. The error of the laser wavelength $\lambda$ was neglected in the calculation of $\delta q$.

To get the error of the thermal energy $T_{e V}=0.5 m_{d}\left\langle v_{x}^{2}\right\rangle$, the error of the particle mass has to be considered. The manufacturer of the particles 45 gives an error of the radius of $\pm 0.09 \mu \mathrm{m}$ for the used particles with radius $a=1.62 \mu \mathrm{m}$. The particle density is $n_{d}=1510 \mathrm{~kg} / \mathrm{m}^{3}$ with an error assumed to be less than $\pm 1 \%$. All this is used in Gaussian propagation of error calculations again.

The results for the relative errors of the average particle velocity and average thermal energy are shown in table 5.2. The calculations are done with the data for $\sigma$ in table 5.1. The impact of the measurement error of the scattering angle $\theta$ can now directly be seen in the relative error of $v_{x}$, and the further increase of the error of $T_{e V}$ can be related to the systematic error of the particle mass $m_{d}$.

The relative errors for the average velocity are between $3 \%$ for $20 \mathrm{~s}$ and up to $25 \%$ for $0.2 \mathrm{~s}$ at angles of $4^{\circ}$ and $9^{\circ}$. The error of $v_{x}$ at $175^{\circ}$ has not increased much because $q$ only depends weakly on $\theta$ near $180^{\circ}$.

The thermal energy has an error between $10 \%$ for $20 \mathrm{~s}$ acquisition and up to $30 \%$ for $0.2 \mathrm{~s}$ acquisition at angles of $4^{\circ}$ and $9^{\circ}$. For measurements with high temporal resolution at $175^{\circ}$ with acquisition times below $0.01 \mathrm{~s}$, an error of the order of $70 \%$ has to be considered. This error can be reduced significantly by using certificated dust particles with smaller standard deviation of to the particle radius.

Besides these main sources of error, there are other effects that can have a negative im-

${ }^{45}$ Visit the manufacturer's website http://www . microparticles . de for details. 
pact on the determination of $T_{e V}$. As already mentioned in the last section, number fluctuations and dust density fluctuations due to phonons on large spatial scales compared to $q^{-1}$ or streaming dust particles can lead to fluctuations in the autocorrelation function. This is shown in figure 5.5 for a $10 \mathrm{~s}$ measurement at $20 \mathrm{~Pa}$ with a scattering angle of $8^{\circ}$. Strong particle streams were present in this measurement. These fluctuations can be averaged, if the acquisition time is sufficiently increased. For short acquisitions, the result of the Gaussian fit can be strongly influenced. The Gaussian fit in figure 5.5 shows a small offset due to the fluctuations. The other fit parameters, in particular the Gaussian decay time, are also affected. These fluctuations are a major contribution to the large errors of $\sigma$ at short acquisition times in table 5.1 .

The amplitude of these fluctuations in the autocorrelation function can also be reduced by probing a larger volume of the particle cloud, considering that this extends the ensemble of particles. But, as already discussed in section 2.2.4, a larger source of light reduces the size of the coherence area as can be seen from equation (2.29) on page 19. The result is a lower amplitude of intensity fluctuations and thus, a lower amplitude of the autocorrelation function peak. Hence, the signal to noise ratio cannot be improved by increasing the scattering volume.

The noise level of the DLS measurements can be defined by the amplitude of the fluctuations in the autocorrelation function, because other effects are negligible. Only for long acquisition times effects like the dark current or gain fluctuations of the photomultiplier, fluctuations of the laser intensity, reflections of unscattered laser light or vibrations in the experimental setup are important sources of noise. To avoid these contributions, the experimental imperfections have to be minimized as discussed in section 4.3.1.

In the following measurements, the errors of the decay time $\sigma$ and the thermal energy $T_{e V}$ will be estimated by the aid of table 5.1 and table 5.2 .

\subsection{Detailed Studies of the Phase Transition}

In this section, the DLS method is applied to study the phase transitions of three-dimensional complex plasmas. The phase transition is induced by variation of the discharge pressure. The thermal energy of the dust particles is measured in the forward scattering regime. The horizontal and the vertical component of movement are detected. The thermal energy is used as a characteristic quantity to describe the phase state.

The phase transitions are analyzed according to a variation of experimental parameters that determine the structure and dynamics of the system. The properties of the phase transition, like the critical pressure, the energy level reached in the disordered state and the anisotropy of the motion of particles in horizontal and vertical direction, are examined. The observations are compared to the predictions of the theory [7, 8, 9] as introduced in section 3.3.2.

The subsections 5.3.2 to 5.3.4 are dealing with the condensation transition. The measure- 
ments therein are performed following the very same procedure. This standard procedure is introduced now shortly.

After trapping as much particles as possible at mediate pressures, the discharge pressure is decreased to cause a melting transition. After that, the pressure is increased again to return to the ordered state. This is repeated three times to avoid particle losses in the melted state in the further procedure.

The pressure is set to the minimum value that serves as the starting point for the condensation transition. The following increase of the discharge pressure is done remotely by a personal computer in discrete steps using the automatic valve 46 . After the desired pressure is reached, the particle system has three minutes to come to equilibrium. Then, the acquisition of the photomultiplier signals of both components is initiated. The acquisition is done with a frequency of $10 \mathrm{MHz}$ and a sample length of $3 \cdot 10^{7}$ points. After the whole pressure sequence is finished, the experimental parameter of interest is changed and the next condensation transition is initiated.

The autocorrelation function is calculated for each data sample and the thermal energy is obtained from the width of the Gaussian fit. The result of the whole procedure is a set of transition curves of the thermal energy $T_{e V}$. Each curve represents one value of the external parameter that was studied.

The position of the scattering volume is chosen to be in the lower third of the dust cloud. Simultaneous CCD observations are performed to verify that the scattering volume is at a constant position within the dust cloud and to analyze the structure and dynamics of the particles system in the vertical plane. The further experimental parameters are the same as described in section 4.1 .

This section is organized as follows. In section 5.3.1, the general appearance of the transition curves for the thermal energy is discussed and an appropriate fit model is introduced. The fit model defines the critical pressure of the phase transition.

In section 5.3.2, the impact of the external bias voltage applied to the driven electrode on the condensing transition is studied. This parameter is essential for the manipulation of the dust cloud and can be used to improve the structural ordering. The influence of the confinement voltages applied to the segments in the lower electrode is also examined in this section.

Section 5.3.3 treats the dependency of the condensation transition on the particle number density. This parameter is hard to control and can have serious effects on the results of a measurement.

In section 5.3.4, condensation for different discharge powers is analyzed. This is a central parameter according to the structure and dynamics of the particle system and a strong impact on the condensation transition can be expected.

Section 5.3.5 is about a fundamental property of phase transitions in complex plasmas under gravity. The gradual melting transition in vertical direction is analyzed by means of DLS.

${ }^{46}$ See experimental section 4.1 for details. 


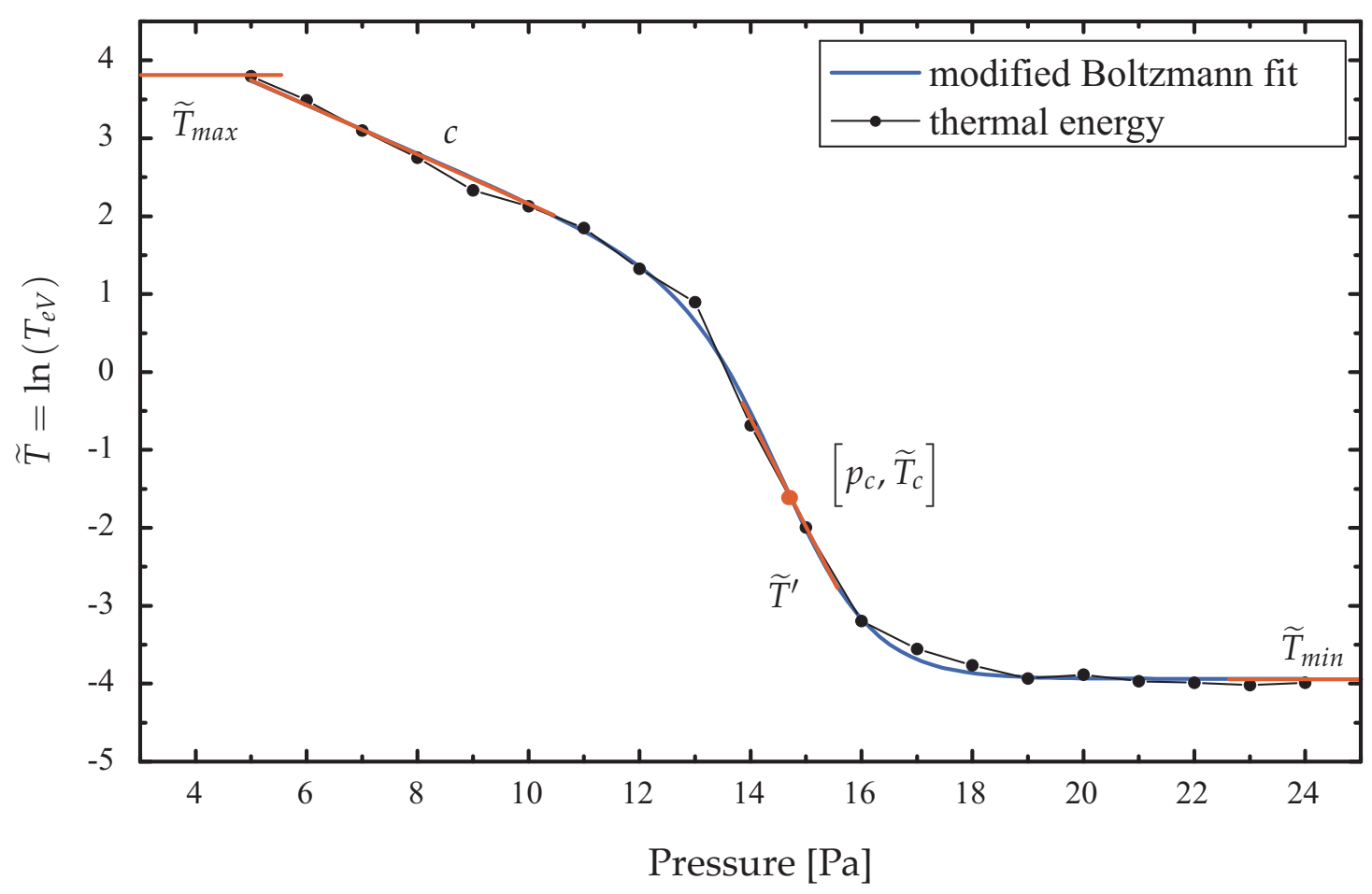

Figure 5.6: A measurement of the thermal energy during a phase transition plotted on a logarithmic scale. The logarithmic data can be fitted by a modified Boltzmann function (equation (5.1)). The parameters to describe the phase transition are the critical pressure $p_{c}$, the corresponding logarithmic energy $\widetilde{T}_{\mathcal{c}}$, the slope at the critical pressure $\widetilde{T}^{\prime}$, the decay constant at low pressures $c$, and the maximum and minimum logarithmic energies $\widetilde{T}_{\text {max }}$ and $\widetilde{T}_{\text {min }}$.

The results are energy maps with discharge pressure and vertical position in the dust cloud as coordinates. These data will be used to analyze gradual progress of energy fronts through the dust cloud.

In section 5.3.6, the anisotropy of the thermal energy in horizontal and vertical direction is studied. The ratio of the energies of the components is examined for variations of different experimental parameters.

Section 5.3.7 shows the melting transition induced by an increase of the discharge power. The features of this kind of transition will be compared to the melting transition induced by pressure reduction.

\subsubsection{The Thermal Energy during a Phase Transition}

The thermal energy of the dust particles during a phase transition, induced by a change of the discharge pressure, shows a characteristic form. A typical measurement of the transition curve of the thermal energy $\widetilde{T}$ is displayed in figure 5.6 on a logarithmic scale. A single-step transition like this is observed for the melting as well as for the condensing transition. The transitions of the horizontal and vertical component show both the same features, but on 
different energy scales. The vertical component reaches about one order of magnitude higher energies.

Simulations on a two-layer system show comparable thermal energies during the melting transition for the horizontal particle motion. But, in contradiction to the measurements in this work, a two-step melting transition in the thermal energy is predicted in [6]. The first step is a growth of energy due to the phonon stream instability, where the structural order is still high. In the second step, the spatial order gets lost and the gaseous state is reached. In contradiction to this, the observations presented here indicate that the rise of energy due to the instability and the structural disorder occur at the same time.

The curve of the thermal energy $\widetilde{T}(p)$ on a logarithmic scale reveals characteristic features like a linear decrease of the thermal energy at low pressures, and a sudden decay at the critical pressure down to energies that correspond to room temperature.

An appropriate fit function has to be introduced, to obtain the characteristic parameters of the curve. The fit parameters allow to compare the transition curves quantitatively, which is in particular important for a large number of transition curves, as in the experiments discussed later.

The "modified Boltzmann" function is introduced here, as a valid function to fit the transition curves:

$$
\widetilde{T}(p)=\widetilde{T}_{m i n}+\frac{\widetilde{T}_{m a x}-\widetilde{T}_{m i n}+c p}{1+\exp \left(\frac{p-p_{c}}{d p}\right)} .
$$

Note that the logarithmic temperatures are used $\widetilde{T}=\ln \left(T_{e V}\right)$ here. The difference to an ordinary Boltzmann function is the linear decrease of $\widetilde{T}$ at low pressures. In this part, the pure Boltzmann function stays on a constant level. The linear part is considered by the additional expression $c p$ in equation (5.1), where $c$ describes the decay rate of $\widetilde{T} . \widetilde{T}_{\text {min }}$ and $\widetilde{T}_{\text {max }}$ represent the minimum and maximum logarithmic energies, $p_{c}$ defines the critical pressure of the transition and $d p$ gives the decay constant at the critical pressure.

The logarithm of the thermal energy at the critical pressure $\widetilde{T}_{c}$ is given by

$$
\widetilde{T}_{c}=\left.\widetilde{T}\right|_{p=p_{c}}=\frac{\widetilde{T}_{\max }+\widetilde{T}_{\min }+c p_{c}}{2} .
$$

The slope at this point is expressed by

$$
\left.\widetilde{T}^{\prime}\right|_{p=p_{c}}=\frac{c}{2}+\frac{\widetilde{T}_{\max }-\widetilde{T}_{\min }+c p_{c}}{4 d p} .
$$

To justify the linear approach at low pressures, a measurement with high resolution was performed. Figure 5.7clearly shows a linear evolution of the logarithmic thermal energy for low pressures.

Some deviations from the linear decrease can be seen around $5 \mathrm{~Pa}$. They can be explained 


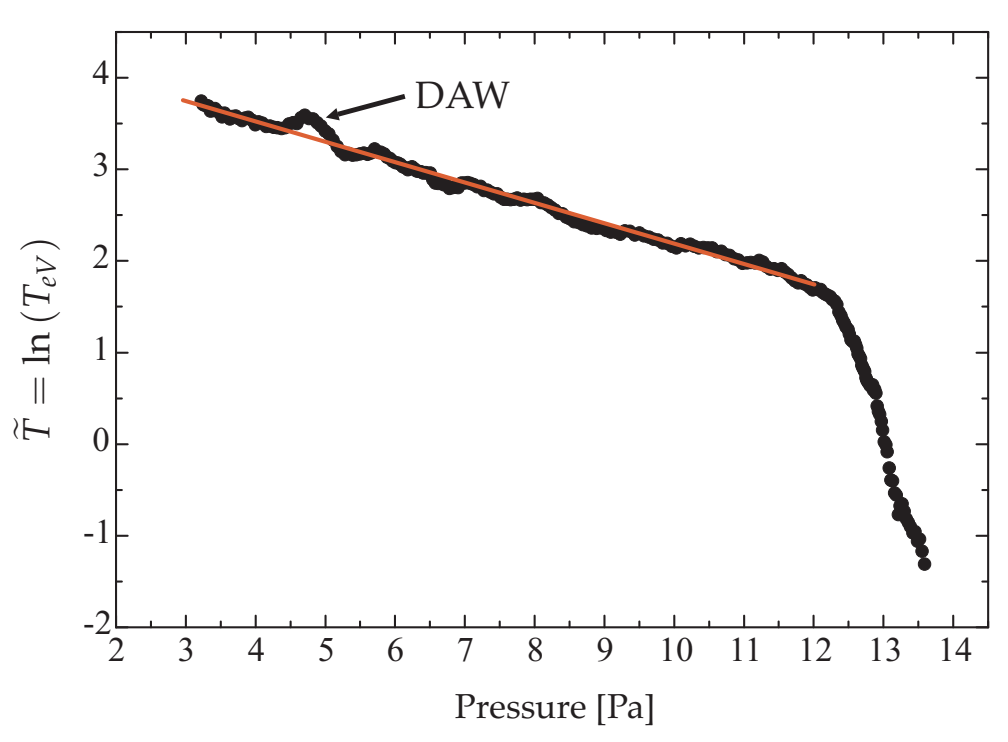

Figure 5.7: A high resolution measurement of the thermal energy at low pressures to justify the linear approach on a logarithmic scale. Deviations from the linear decrease at $5 \mathrm{~Pa}$ are caused by dust acoustic waves (DAW). The impact of the DAW is not averaged due to the short sample time.

by dust acoustic waves (DAW) that are present in this range of pressure. The acquisition time was chosen to be short to get a sufficient high resolution, and therefore the impact of the dust acoustic waves is not averaged.

In the following the critical pressure will be denoted by $p_{\text {cond }}$ for condensation transitions and $p_{\text {melt }}$ for melting transitions.

\subsubsection{External Bias Potential and Confinement Voltage}

Two of the most important experimental parameters to manipulate the dust cloud are given by the confinement voltages and an external bias voltage. As shown in figure 5.8 , the external bias voltage is directly applied to the driven electrode by a decoupling circuit. Hence, no selfbias potential can evolve freely, but the electrode potential is fixed. An external DC voltage up to $\pm 50 \mathrm{~V}$ can be applied for the common plasma parameters in this experiment. The plasma cannot be driven stable for higher voltages, because arcing between the powered surface and the grounded edge of the electrode appears.

The external bias potential can be used to change the vertical position of the whole dust cloud. The stability of vertical particle chains is affected by this voltage too. This way of particle manipulation has a global character, inasmuch as the plasma is changed as a whole.

The confinement voltages are applied to the segments of the lower electrode, as depicted in figure 5.8. The potential well for particle trapping can be manipulated by voltages on an outer ring, a ring around the center segment, the centered segment itself, and two additional small rings (see section 4.1 for details). In the experiments discussed here, all segments of the lower electrode were set to the same voltages between $+15 \mathrm{~V}$ and $-20 \mathrm{~V}$ by means of a multichannel, decoupled DC voltage supply. The confinement voltages change the potential well only locally. By changing the voltage at the centered segment, the dust cloud is deformed mainly above the segment. It is important to avoid big potential steps from one to another 


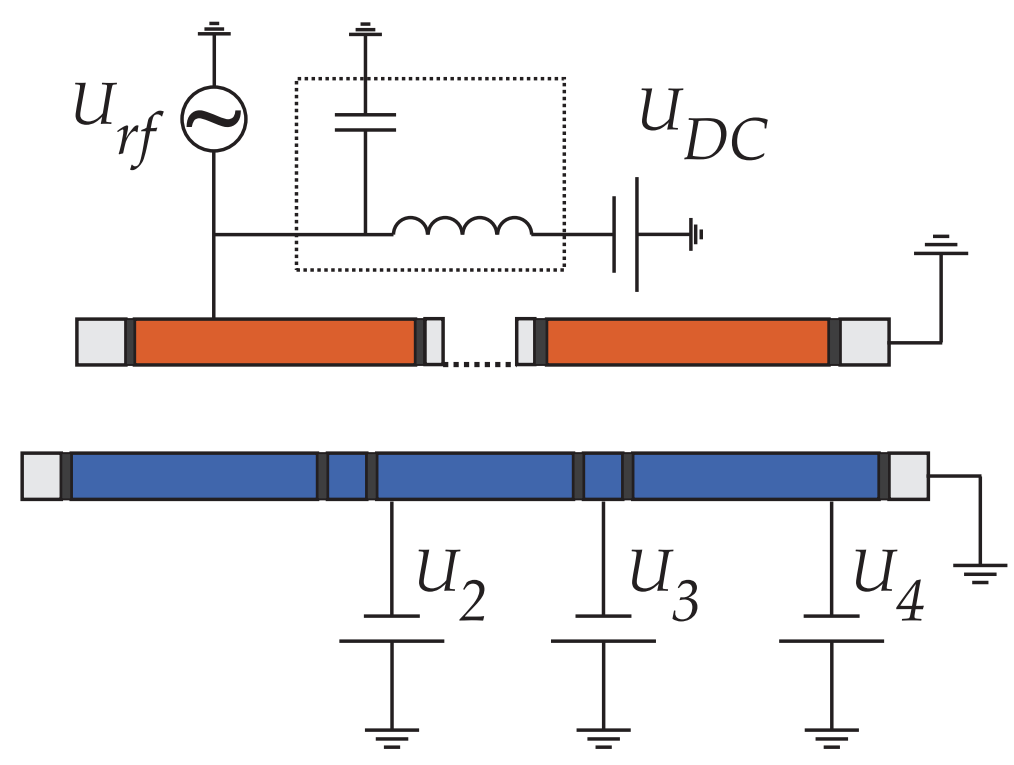

Figure 5.8: Cross section of the upper and the lower electrode. The inner segment of the upper electrode is powered with $U_{R F}$. The external bias voltage $U_{D C}$ is applied by a decoupling circuit (dashed box) on the powered segment. The lower electrode has five segments (segments 1 and 5 are not shown in this cross section) and voltages $U_{1}$ to $U_{5}$ can be applied for dust confinement and manipulation. The voltages are applied to the segments by a multichannel, decoupled DC voltage supply. For the purposes of simplified illustration, the electrodes are drawn closer to each other as they are in the experiment.

segment. They can result in collective vortex motion of the dust particles near a potential step [90].

Both, the external bias voltage and the confinement voltage are supposed to affect structural ordering of the dust particles, as well as influencing the damping of the ion two-stream instability and the phonon stream instability, respectively. Therefore, the impact of the external voltages on the phase transition can play a crucial role, and voltage fluctuations can lead to strong statistical deviations in the results of the measurements.

To study the impact of the external bias voltage, the condensation transition is examined for negative and also for positive voltages, following the standard procedure described in the introduction of the section 5.3. Simultaneous CCD observations were used to keep the parameters of the dust cloud as constant as possible. Namely, the size of the cloud and the position of the scattering volume within the dust cloud were controlled.

Negative bias voltages between $0 \mathrm{~V}$ and $-40 \mathrm{~V}$ were studied in a first measurement. The case of floating bias (self-bias) potential was also treated in this measurement. At self-bias conditions, the upper electrode is isolated for DC fields and it cannot discharge to ground. The self-bias changed during the pressure variation from $-25 \mathrm{~V}$ at $5 \mathrm{~Pa}$ to $-15 \mathrm{~V}$ at $24 \mathrm{~Pa}$. Positive bias voltages between $5 \mathrm{~V}$ and $35 \mathrm{~V}$ were examined in a second experiment.

Figure 5.9 presents the thermal energy of the horizontal component of motion versus the discharge pressure. Figure (a) shows the negative bias voltages and self-bias conditions. The 
(a)

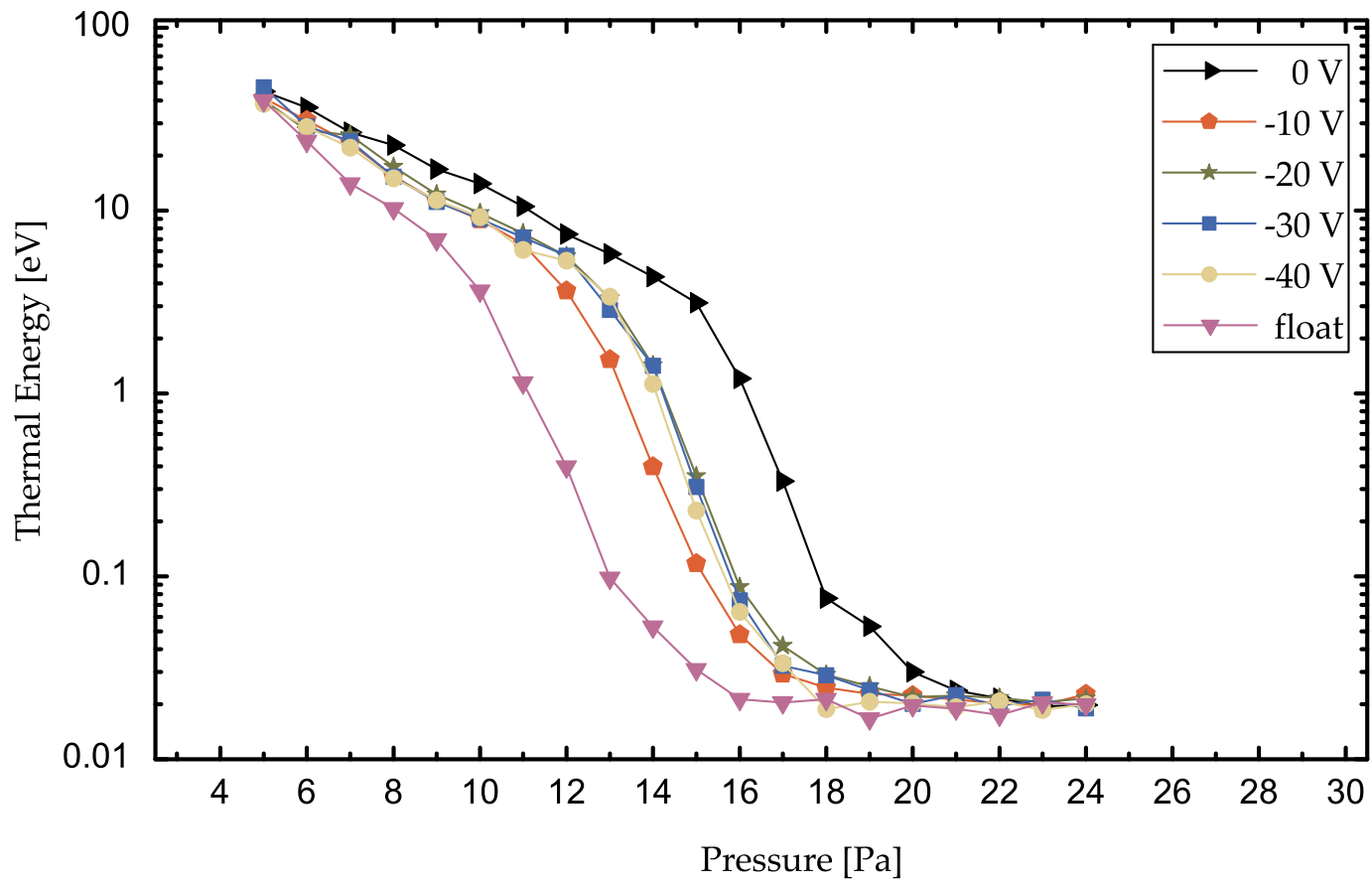

(b)

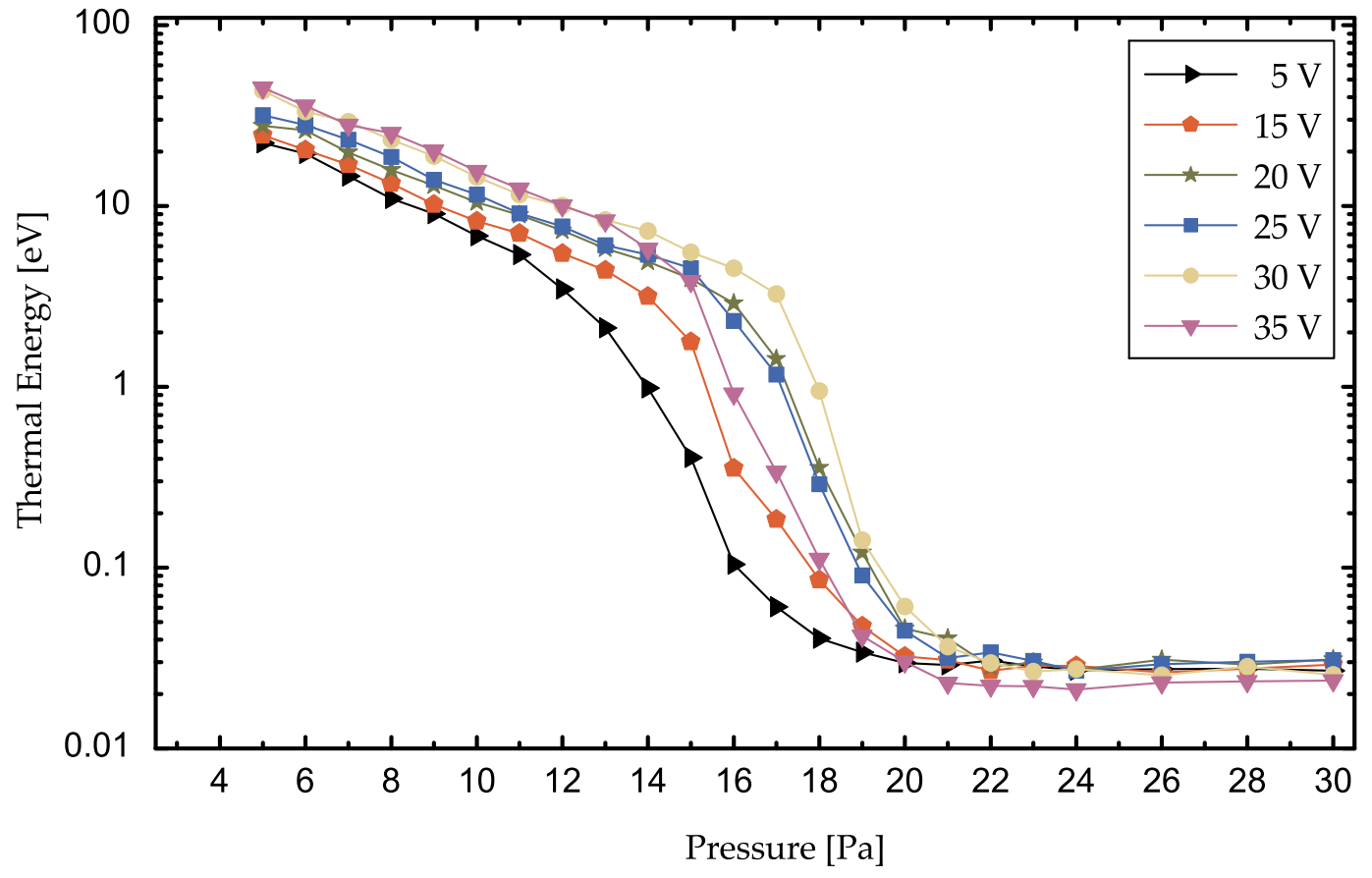

Figure 5.9: Condensation transition for negative (a) and positive (b) external bias voltages. The pressure was increased in discrete steps from $5 \mathrm{~Pa}$ to above $24 \mathrm{~Pa}$. The thermal energy falls from about 50 $\mathrm{eV}$ to nearly room temperature. The condensation transition can be strongly affected by the external bias voltage. 
measurement with self-bias is denoted with "float". In figure (b), the positive bias voltages are shown. The transition curves of the thermal energy for the vertical direction look very similar and are not shown here. One notable difference is a thermal energy of about $500 \mathrm{eV}$ in the vertical direction at a discharge pressure of $5 \mathrm{~Pa}$ compared to $50 \mathrm{eV}$ in the horizontal direction. The general scale between the energies of vertical and horizontal component in the melted state is a factor of 10 .

The typical transition of the thermal energy is observed and a detailed analysis of the condensation curves can be done with the modified Boltzmann fit as introduced in the last section. Figure 5.10 depicts the fit parameters to account the impact of the external bias potential on the condensation transition. The figures (a) and (b) refer to the first measurement with negative bias voltages, and figures (c) and (d) are according to the positive voltages. On the left side, the critical pressure for condensation $p_{\text {cond }}$ is plotted versus the external bias voltage. The figures on the right side show the decay rate of the exponential decay of the thermal energy at low pressures. This corresponds to the parameter $c$ of the modified Boltzmann function. The measurement with self-bias conditions is shown separately in the upper figures on the left side. The horizontal component (closed symbols) and the vertical component (open symbols) are analyzed by the aid of the modified Boltzmann fit.

Two ranges can be distinguished for negative external bias voltages. The critical pressure for condensation $p_{\text {cond }}$ strongly varies for voltages between $0 \mathrm{~V}$ and $-20 \mathrm{~V}$. For voltages in the range of $-20 \mathrm{~V}$ and $-40 \mathrm{~V}$ the critical pressure is about $15.5 \mathrm{~Pa}$ and does not change very much.

The same is true for the decay constant $c$. Figure (b) shows, $c$ is stable at $-0.24 \mathrm{~Pa}^{-1}$ for voltages in the range of $-20 \mathrm{~V}$ and $-40 \mathrm{~V}$. This is confirmed by CCD observations too. The dust cloud changes its vertical position and shape for low negative voltages, but for voltages above $-20 \mathrm{~V}$, a stable regime is observed. The situation with self-bias conditions is different compared to the case of fixed external voltages. As can be seen with the naked eye, the plasma distribution is changed. The critical pressure for condensation $p_{\text {cond }}$ for positive external bias voltages (figure 5.10 (c)) rises for an increase of the voltage from $5 \mathrm{~V}$ to $20 \mathrm{~V}$. A stable region is found between $20 \mathrm{~V}$ and $30 \mathrm{~V}$. In this range $p_{\text {cond }}$ is about $18 \mathrm{~Pa}$ and changes only a few. For higher voltages $p_{\text {cond }}$ drops again. Video observations show that the dust cloud is lifted by the positive voltage until $20 \mathrm{~V}$ is reached. Then the position does not change anymore. The plasma distribution changes significantly for voltages above $35 \mathrm{~V}$ and the dust cloud is at a lower position. The plasma emission at the sheath edge is seen to be higher too.

The stable range between $20 \mathrm{~V}$ and $30 \mathrm{~V}$ is also found for the decay constant $c$ as can be seen in figure (d). Only the vertical component seems to show a slightly increased decay constant due to the change in the vertical electric field.

The critical pressure is in general higher for the vertical component for all applied voltages, but both components show a similar behavior.

The second important manipulation voltage, the confinement voltage, is studied in the same way. Figure 5.11 shows the transition curves of the thermal energy versus discharge 
(a)

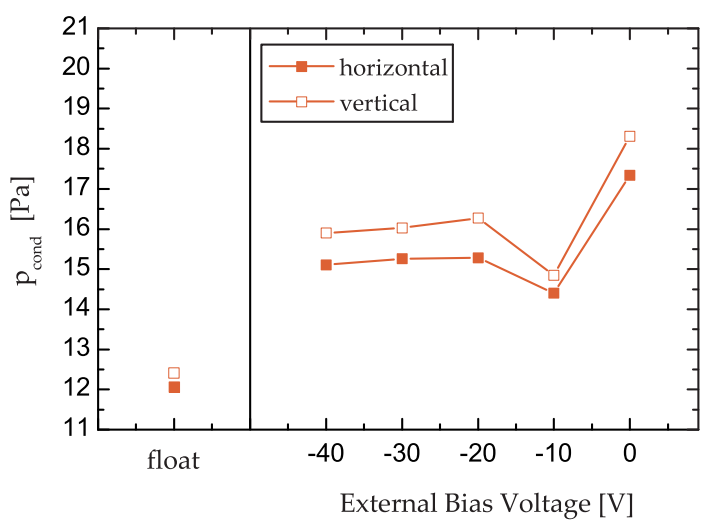

(c)

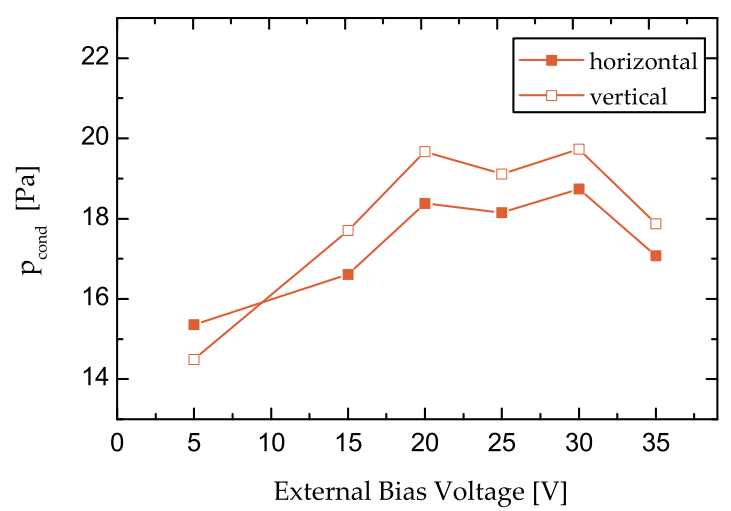

(b)

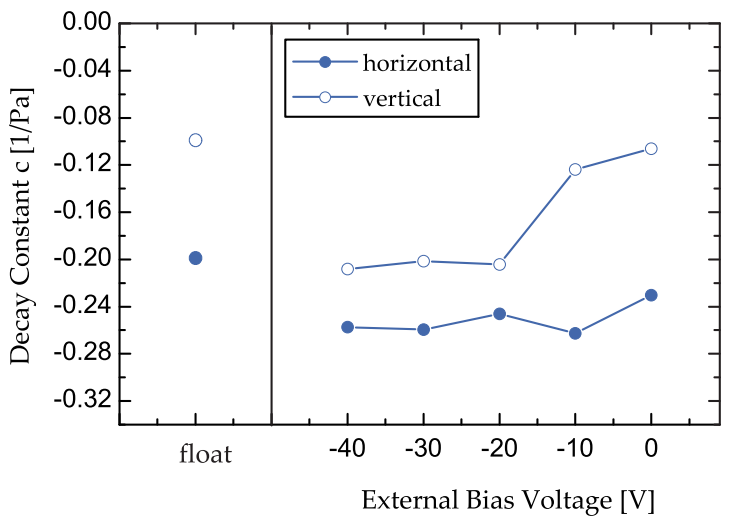

(d)

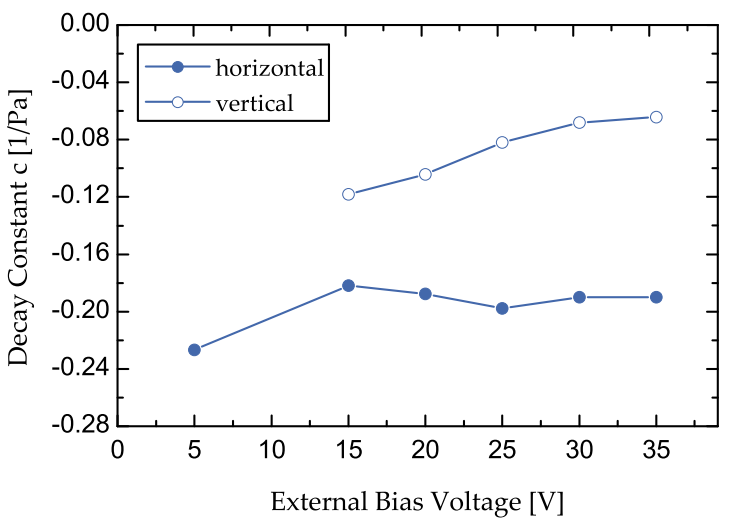

Figure 5.10: The critical pressure $p_{\text {cond }}((\mathrm{a})$ and $(\mathrm{c}))$ and the decay constant $c((\mathrm{~b})$ and $(\mathrm{d}))$ are plotted versus the external bias voltage. Both parameters are obtained from the modified Boltzmann fit, which was applied to the transition curves in figure 5.9. A range of stable conditions is found for negative as well as for positive external bias voltages.

pressure for different confinement voltages between $15 \mathrm{~V}$ and $-20 \mathrm{~V}$. The impact of the confinement voltage is negligible, as apparent from the overlapping curves. Some irregularities appear in the vertical component due to dust acoustic waves (DAW), as will be discussed later in this section.

It might be surprising that particle trapping can be realized with negative voltages applied to the segments of the electrodes. This is possible for negative external bias voltage at the same time. The repulsive confinement voltage can be compensated by this. Furthermore, the plasma experiment as designed here shows a certain self-confinement. That is, even without confinement voltages, a small dust cloud can be trapped.

The independence of the condensation transition on the confinement voltage emphasizes on the different character of this voltage compared to the external bias voltage. The plasma does not change much as a whole due to a change of the confinement voltage. In addition, 
(a)

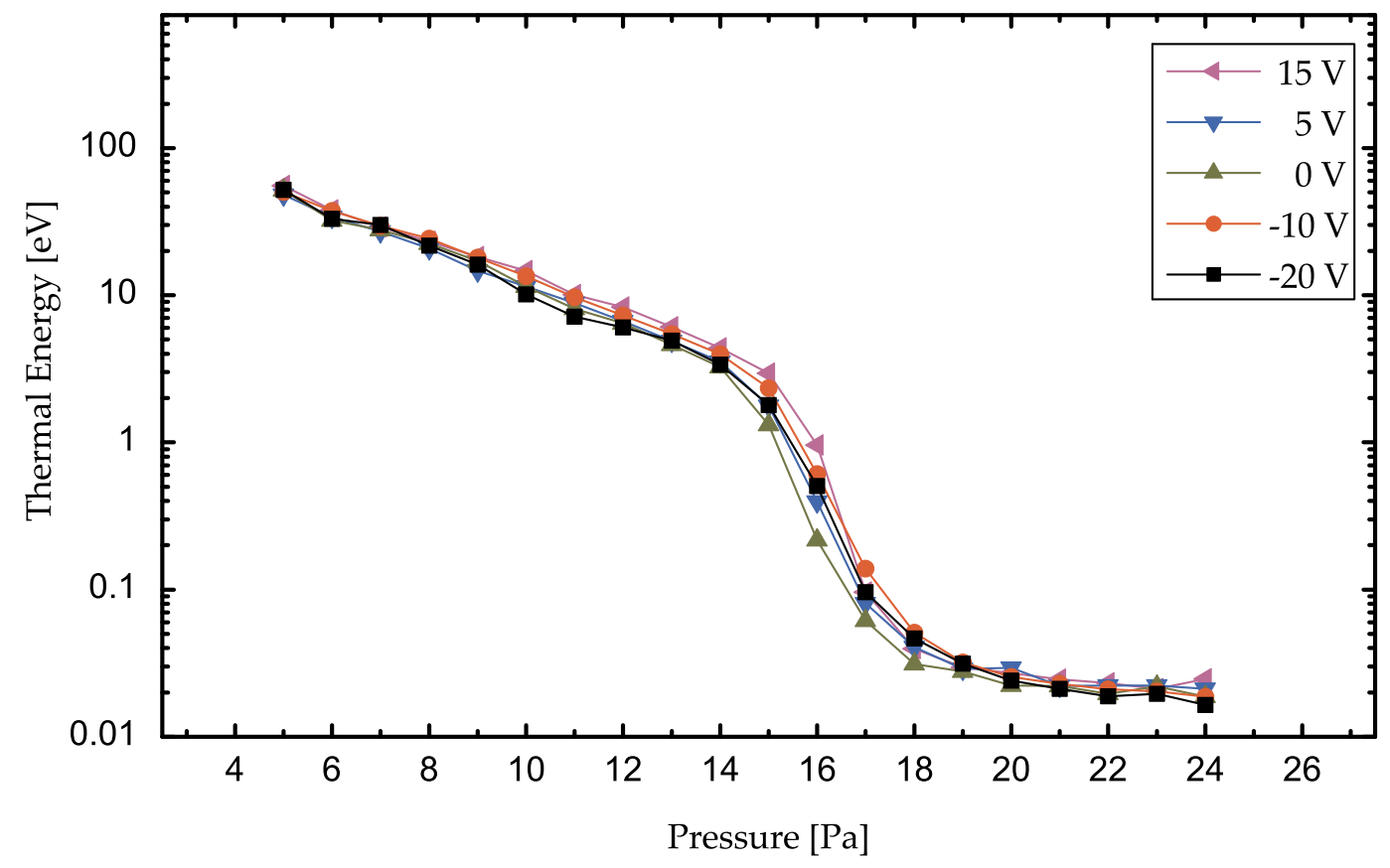

(b)

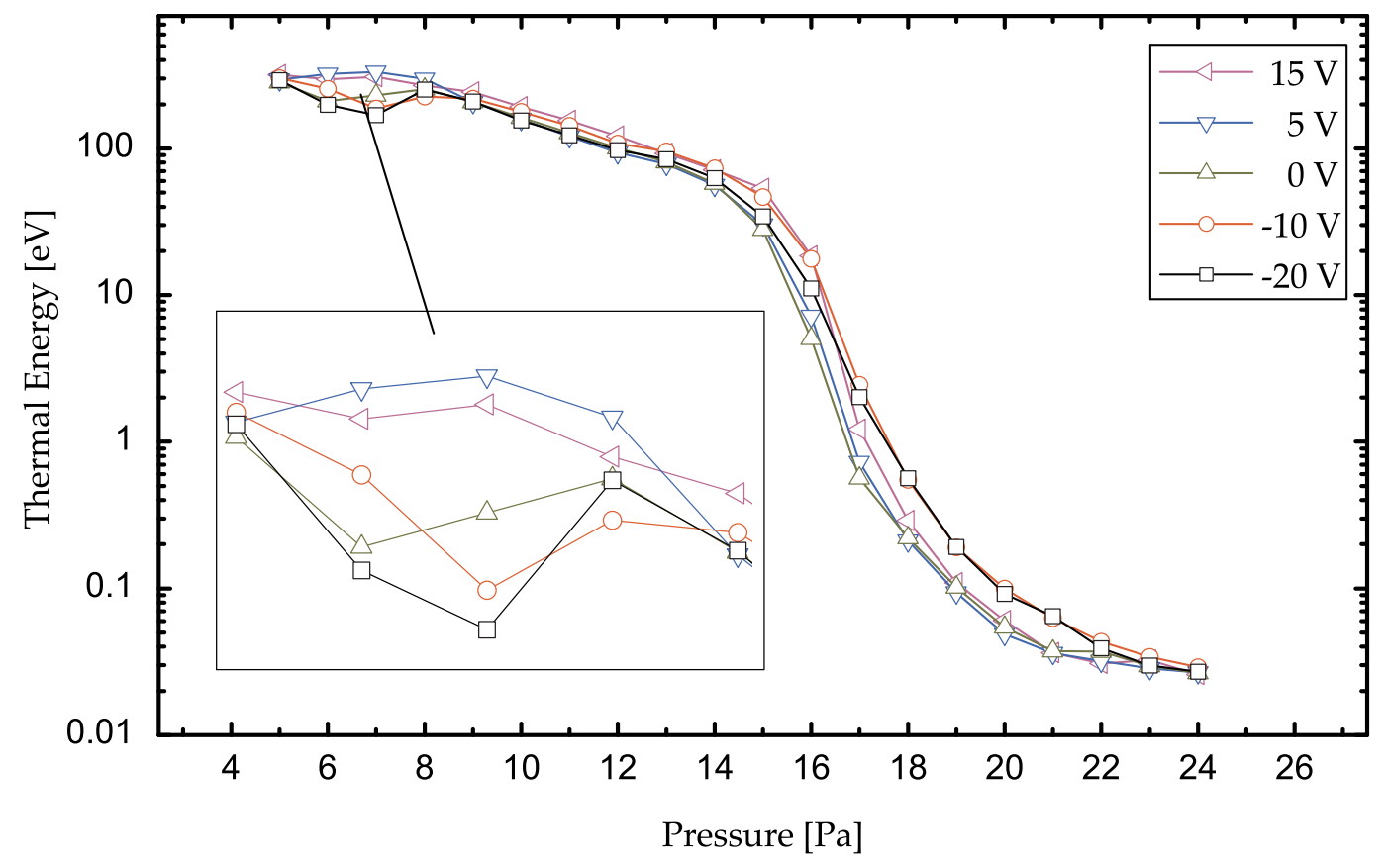

Figure 5.11: Condensation transition for confinement voltages between $-20 \mathrm{~V}$ and $15 \mathrm{~V}$ applied to the segments in the lower electrode. The horizontal component (a) is undisturbed by the change of the confinement voltage. This is also true for the vertical component (b). A slight irregularity can be seen for the vertical component. This is due to energy losses in the vicinity of the lower electrode in combination with dust acoustic waves. 
(a)

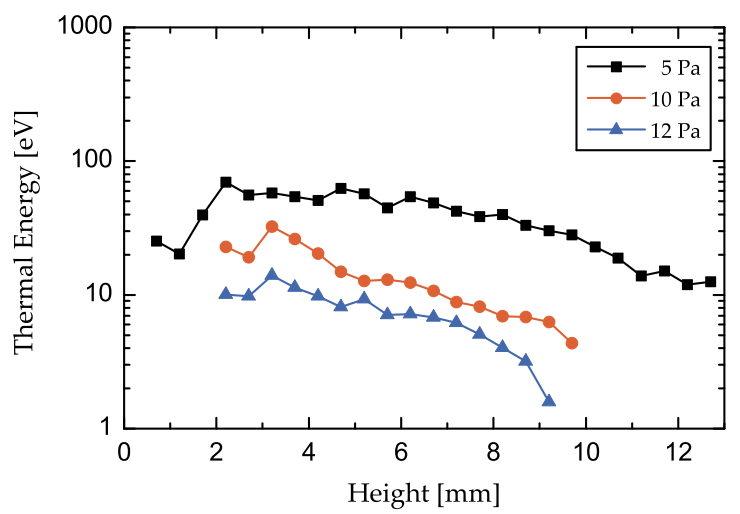

(b)

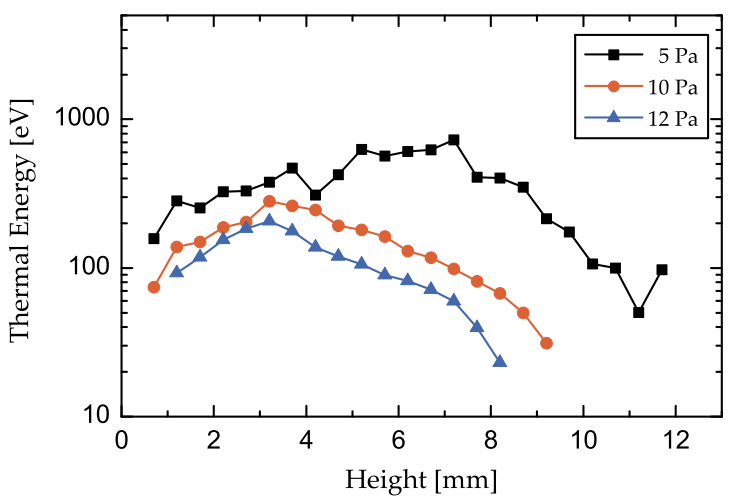

Figure 5.12: The thermal energy for different vertical positions in the dust cloud at pressures of $5 \mathrm{~Pa}$, $10 \mathrm{~Pa}$ and $12 \mathrm{~Pa}$. The particle system was in the melted state and dust acoustic waves were present. For the horizontal component (a), the thermal energy increases nearly linearly toward the electrode. The vertical component (b) starts to decrease suddenly $3 \mathrm{~mm}$ in front of the electrode for $10 \mathrm{~Pa}$, and $12 \mathrm{~Pa}$. This decrease already starts at $8 \mathrm{~mm}$ for $5 \mathrm{~Pa}$. The nonlinear sheath near the lower electrode is cooling the vertical dust particle motion and dust acoustic waves are an additional mechanism of heat transport at $5 \mathrm{~Pa}$.

this means no compression of the dust cloud is observed because the condensation should be affected by this.

On the basis of this analysis, we choose the experimental parameters in subsequent measurements to give maximum of stability. This analysis also demonstrates the stability of the experimental conditions. The results are reproducible, even after several melting and condensation transitions were performed.

The irregularities at pressures around $6 \mathrm{~Pa}$ in figure 5.11(b) are due to large amplitude dust acoustic waves. Note, the thermal energy is measured over several cycles of the dust acoustic wave, and therefore, variations of the velocities in different phases of the wave are averaged. CCD observations show that the dust particles come very close to the lower electrode due to the oscillation into the vertical direction. A possible explanation for the drop of the thermal energy is the nonlinearity of the sheath in the vicinity of the electrode. Here, the particles find very different conditions and it cannot be assumed, that the heating processes are the same. Interestingly, the affect on the horizontal motion is much lower. That speaks for a low coupling between both components of motion.

A more detailed study of the losses of energy near the lower electrode is done by vertical scanning through the dust cloud by DLS. The position of the scattering volume is varied gradually from the top of the dust cloud to the bottom. Figure 5.12 illustrates the result. The thermal energy is plotted as a function of the vertical coordinate for (a) the horizontal and (b) the vertical component. Three discharge pressures of $5 \mathrm{~Pa}, 10 \mathrm{~Pa}$ and $12 \mathrm{~Pa}$ were analyzed. The particle system was in the melted state and dust acoustic waves were present. 
The thermal energy in horizontal direction (a) drops almost linearly with an increase of the height of the scattering volume for all three pressures. Only a few points near the lower edge of the cloud show reduced energies. This might be due to a lower particle density, i.e. the scattering volume was not completely filled with dust.

The thermal energy in the vertical direction (b) on the other hand suddenly drops in the vicinity of the lower electrode. The position of the drop depends on the discharge pressure. For pressures of $10 \mathrm{~Pa}$ and $12 \mathrm{~Pa}$, the thermal energy starts to drop at about $3 \mathrm{~mm}$ above the lower electrode. For $5 \mathrm{~Pa}$, the drop is already at $8 \mathrm{~mm}$. Thus, there seems to be some source of cooling originating from the vicinity of the lower electrode. The range of this cooling is longer at low pressures. This might be due to the presence of dust acoustic waves giving an additional heat transport mechanism that leads to cooling of the upper particles. The larger amplitudes of the dust acoustic waves at lower pressures increases the range of energy transport.

The observations of the condensation transition presented in this section are in agreement with the theory about the ion-two stream instability by Joyce et al. [7, 8, 9]. The variation of the external bias voltage in particularly changes the position of the sheath edge, and the width of the entire sheath. This results in a different ion stream velocity $u_{i}$. This has impact on the stability condition for the ion-two stream instability (equation (3.43)). Moreover, the ion collision rate $v_{i}$ is changed, because it is proportional to $u_{i}$. This affects the damping of the instability too.

These are examples how the observations can be explained qualitatively. Of course, a change of the sheath conditions affects plenty of other parameters, like the dust charge, the wake field, and therefore the structure of the dust cloud and the dimensions of the dust particle system.

The independence of the condensation transition on the confinement voltage is due to the local character of this manipulation voltage. No change of the sheath edge position or the sheath width is observed due to a variation of the confinement voltage. The global plasma parameters are nearly undisturbed.

\subsubsection{Particle Number Density and Height of the Dust Cloud}

The particle number density $n_{d}$ is an experimental parameter that is hard to control. The injection of particles by a dust dispenser, as discussed in the experimental section 4.1, gives only a rough control over the amount of dust that is introduced into the plasma. Besides the number density, the height of the particle cloud $h_{c}$ is assumed to have a strong impact on the condensation transition [7, 8, 9].

The impact of these parameters is studied by a measurement following the standard procedure that is described in the introduction of the section 5.3. The size of the dust cloud was reduced after each condensation transition by pushing a part of the particles over the edge of 


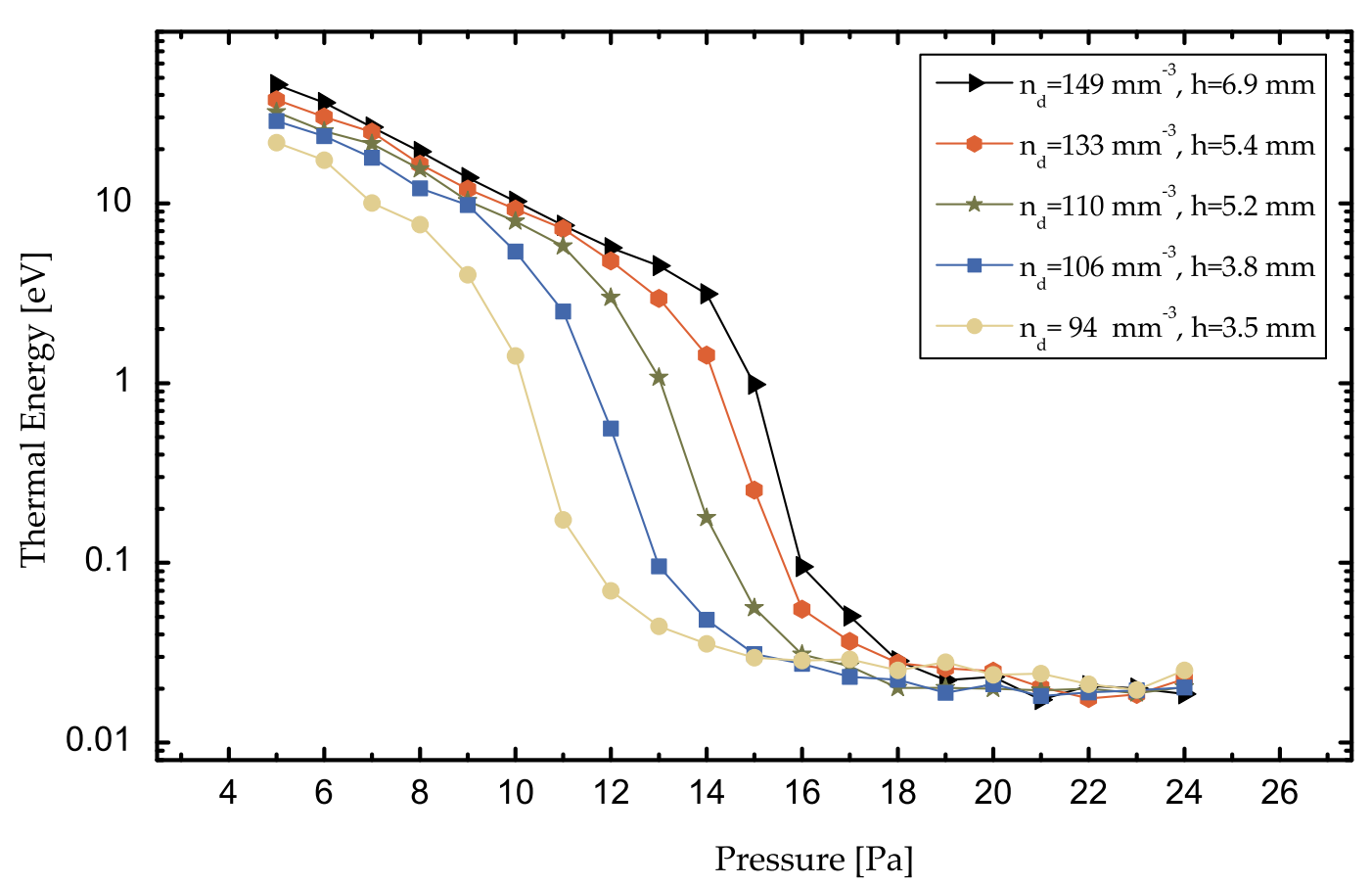

Figure 5.13: Thermal energy of the horizontal component of movement versus the discharge pressure under variation of the particle number density $n_{d}$ and the height of the dust cloud $h_{c}$. For lower values of $h_{c}$ and $n_{d}$, the condensation curves are shifted to lower pressures and the maximum of energy attained in the melted state is lower.

the lower electrode. This was achieved by means of the confinement voltages.

The height of the dust cloud and the number density were determined by simultaneous CCD observation of a vertical plane. To calculate the number density, the average inter-particle separation $\Delta$ is determined by analysis of the pair correlation function $g(r)$. Even though $\Delta$ is the average particle distance in the vertical plane, it is used to calculate a three-dimensional particle density via $n_{d}=\left(4 / 3 \pi \Delta^{3}\right)^{-1}$. The CCD measurement was done in the ordered state, and $n_{d}$ is therefore only an estimate for the particle density during the phase transition. The particle density in the melted state has to be considered to be somewhat lower.

Figure 5.13 shows the thermal energy in the horizontal plane versus the discharge pressure for different particle densities $n_{d}$ and heights of the particle cloud $h_{c}$. The condensation transition shifts to lower pressures as the density is reduced from $n_{d}=149 \mathrm{~mm}^{3}$ to $n_{d}=94 \mathrm{~mm}^{3}$ with a corresponding change of the dust cloud height from $h_{c}=6.9 \mathrm{~mm}$ to $h_{c}=3.5 \mathrm{~mm}$. The maximum energy, reached at low discharge pressures, decreases with the particle number density and height of the cloud. The decay rate of energy toward the transition pressure is nearly the same for all conditions examined here.

The data in figure 5.13 are analyzed by the modified Boltzmann fit. The critical pressure for condensation $p_{\text {cond }}$ gives insight into the impact of particle density and height of the cloud on the transition. In figure 5.14, the critical pressure versus the product of the particle number 
Figure 5.14: The critical pressure for condensation $p_{\text {cond }}$ versus the product of height of the dust cloud and particle number density $n_{d} \cdot h_{c}$. The critical pressure $p_{\text {cond }}$ strongly drops for low values of $n_{d} \cdot h_{c}$. The measurement indicates a saturation at values above $800 \mathrm{~mm}^{-2}$, the impact of $n_{d} \cdot h_{c}$ is less important.

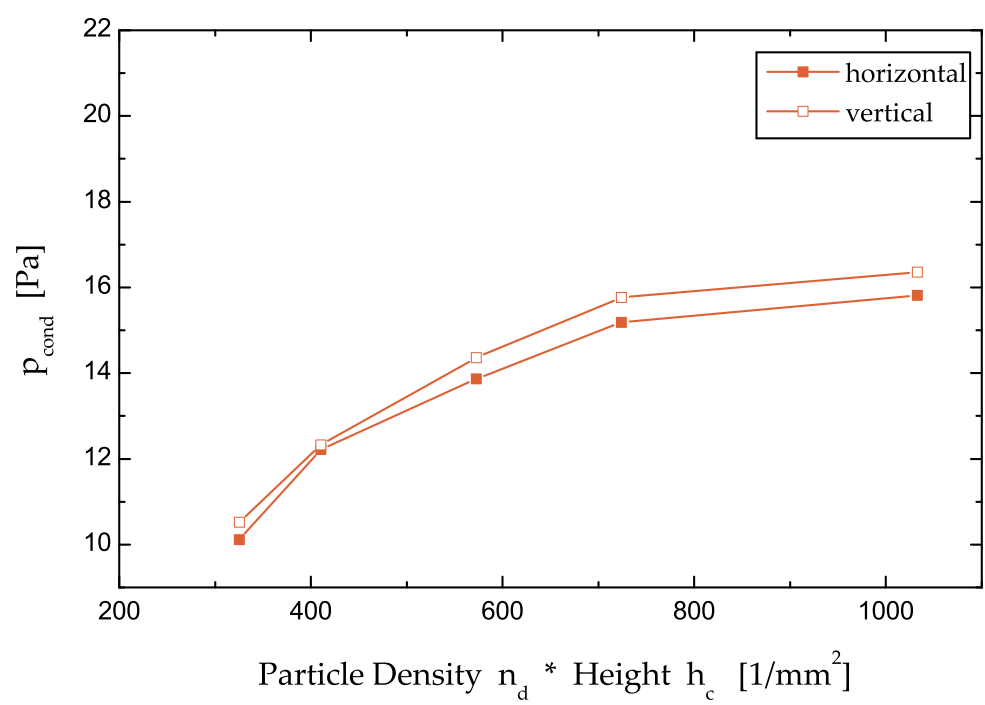

density $n_{d}$ and the height of the dust cloud $h_{c}$ is shown. The horizontal and vertical particle motion are analyzed.

The critical pressure $p_{\text {cond }}$ rises with increase of the product $n_{d} \cdot h_{c}$. The increase is stronger at low values and a saturation is indicated at high values of $n_{d} \cdot h_{c}$. The same dependency is observed for the horizontal and vertical particle movement. This result implies that, if the dust cloud is sufficiently extended in the vertical direction and the number density is large enough, the impact of the particle density on the condensation transition is weak.

The role of $n_{d}$ and $h_{c}$ as observed in this experiment is consistent with the theory by Joyce et al. [7, 8, 9]. An increase of $n_{d}$ leads to a higher dust plasma frequency $\omega_{d}$, because it is proportional to $\sqrt{n_{d}}$. This results in a decrease of the ratio of dust-neutral collision rate and dust plasma frequency $v_{d} / \omega_{d}$. To maintain the damping of the ion-two stream instability, the decrease of $v_{d} / \omega_{d}$ has to be compensated by a higher collision rate $v_{d}$, i.e. a higher gas pressure is necessary. Thus, the critical pressure $p_{\text {cond }}$ grows with $n_{d}$.

Then again, the boundary condition (right hand side of equation (3.43) itself depends on $\omega_{d}$ and therefore on $\sqrt{n_{d}}$, albeit it is a weaker dependency. An increase of $n_{d}$ shifts the boundary to higher values. This also has to be compensated by a higher collision rate $v_{d}$, i.e. an even higher critical pressure $p_{\text {cond }}$.

In the publications [7, 8, 9], it was noted, that the ion-two stream instability shows to be convective and the height of the dust cloud determines the critical pressure of condensation and the heating rate due to the instability. A larger height of the dust cloud is expected to shift the critical pressure $p_{\text {cond }}$ to higher values, considering that the dust ensemble is more sensitive to the ion-two stream instability. 


\subsubsection{Discharge Power and Particle Size}

The discharge power is an elementary plasma parameter that has an effect of the density and the temperature of electrons and ions in the plasma. A variation of the discharge power has serious consequences on structure and dynamics of the dust ensemble. To shed light on the impact of this parameter on the phase transition in a complex plasma, the condensation transition is studied under variation of the discharge power in this section. The results will be compared to the predictions of the theory. Two experiments with particles of different size are performed to sustain the theoretical interpretations.

The thermal energy was measured for different discharge powers, again following the standard procedure. The discharge power was varied between $1 \mathrm{~W}$ and $22 \mathrm{~W} 47$. These values correspond to peak-to-peak voltages between $35 \mathrm{~V}_{\mathrm{pp}}$ and $80 \mathrm{~V}_{\mathrm{pp}}$. This was measured by a calibrated voltage probe with an error of $\pm 5 \%$. After a change of power, the matching was adapted to the changed parameters. Two different particle diameters of $3 \mu \mathrm{m}$ and $7 \mu \mathrm{m}$ were examined in two independent experiments. The other experimental parameters were kept the same, except for the confinement voltage. The confinement voltage was $40 \mathrm{~V}$ for $3 \mu \mathrm{m}$ particles and $30 \mathrm{~V}$ for $7 \mu \mathrm{m}$ particles to ensure the most effective particle trapping. This difference should not affect the comparability of the results, because the impact of the confinement voltage was shown to be small in section 5.3.2. The external bias voltage was chosen to be 19 V. Regarding to the observations in section 5.3.2, the impact of the external bias voltage is reduced in this voltage range.

Figure 5.15 (a) depicts the critical pressure for condensation $p_{\text {cond }}$ as obtained from the modified Boltzmann fit of the transition curves of the thermal energy $T_{e V}$. The horizontal and vertical components of motion are analyzed for both particle sizes. In (b), the product of height and particle number density $n_{d} \cdot h_{c}$ is plotted versus the discharge power. Again, the CCD technique was used to measure the height and particle density in the ordered phase state.

The critical pressure $p_{\text {cond }}$ shows a similar behavior for both sizes of particles. A rise of $p_{\text {cond }}$ is observed at low discharge powers, and $p_{\text {cond }}$ changes less and finally saturates for higher discharge powers. The curve of the $7 \mu \mathrm{m}$ particles is shifted to lower values compared to the $3 \mu \mathrm{m}$ particles. The vertical component condensates at slightly higher pressures than the horizontal component for both particle sizes.

A change of the discharge power involves a variation of the sheath width and sheath edge position. The major effect among others on the dust particle system is a change of the particle number density and the height of the dust cloud. As shown in figure 5.15 (b), the product of density and height in general increases with the discharge power. For $3 \mu \mathrm{m}$ (squares) particles, a nearly constant value of $n_{d} \cdot h_{c}$ is seen for low powers. Above $10 \mathrm{~W}$ a higher level of $n_{d} \cdot h_{c}$ is reached. For $7 \mu \mathrm{m}$ (circles) particles, an increase is found that gets slightly weaker

${ }^{47}$ These values are the output powers as displayed from the generator. 
(a)

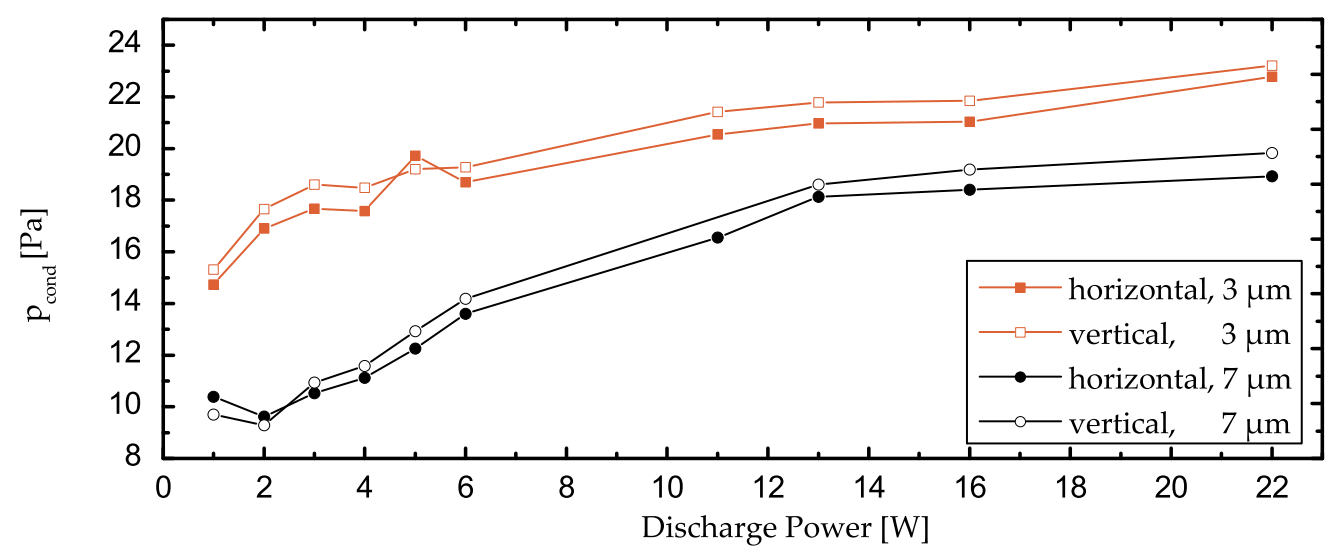

(b)

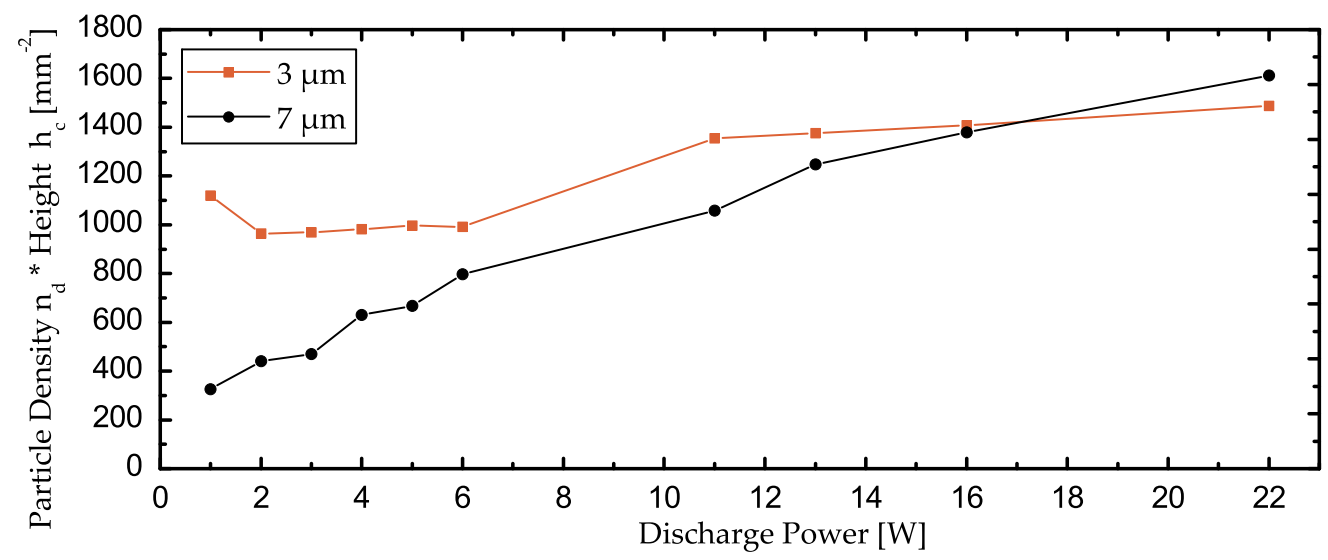

Figure 5.15: (a) Impact of the discharge power on the critical pressure for condensation $p_{\text {cond }}$ for $3 \mu \mathrm{m}$ particles (squares) and $7 \mu \mathrm{m}$ particles (circles). The horizontal (closed symbols) and the vertical component (open symbols) of motion are shown. (b) The product of particle density and height of dust cloud $n_{d} \cdot h_{c}$ versus discharge power. To determine $n_{d}$ and $h_{c}$, simultaneous CCD measurements were performed.

for higher powers.

The results shown in figure 5.14 imply that the condensation transition is only weakly affected for values of $n_{d} \cdot h_{c}$ above a certain threshold. This also becomes visible in the evolution of $p_{\text {cond }}$ in figure 5.15 (a). For high powers, the product of $n_{d} \cdot h_{c}$ is large and $p_{\text {cond }}$ is nearly constant. This is true for both particle sizes. For the $7 \mu \mathrm{m}$ particles, a similar increase for $p_{\text {cond }}$ and $n_{d} \cdot h_{c}$ is seen at low discharge powers. Here, the strong dependency of $p_{\text {cond }}$ on $n_{d} \cdot h_{c}$ for low densities and heights becomes obvious again. The condensation pressure $p_{\text {cond }}$ for $3 \mu \mathrm{m}$ particles at low powers slowly drops similar to the quantity $n_{d} \cdot h_{c}$. The product of density and height is still quite large, so $p_{\text {cond }}$ changes only little.

Note, the situation at $1 \mathrm{~W}$ looks quite different. From visual observations, it can be seen that 
the plasma distribution changes seriously below discharge powers of $2 \mathrm{~W}$. The particle cloud is not as stable as it is for higher powers. So, one has to be careful with the interpretation of the measurement at $1 \mathrm{~W}$.

The dependency of the condensation transition as described in the simulations by Joyce et al. [7, 8, 9] on the particle number density and height was already discussed in the last section. An increase of $p_{\text {cond }}$ for higher $n_{d} \cdot h_{c}$ is predicted. This is expected to give the main contribution to the increase observed in figure 5.15 (a).

There are other parameters that have to be discussed regarding the theoretical predictions. Firstly, the ion density $n_{i}$ rises with the discharge power and it follows a higher ion plasma frequency. After equation (3.43), the boundary gets shifted to lower values and the ratio $v_{i} / \omega_{i}$ reduces too. Both has to be countered by a higher neutral gas pressure to maintain the damping of the ion-two stream instability. Hence, the critical pressure is expected to rise with discharge power. This is another contribution to the growth observed in figure 5.15 (a).

Secondly, the electron temperature $T_{e}$ has to be regarded. Even though an increase of the discharge power raises mainly the electron density, the temperature is also affected. A larger $T_{e}$ leads to a higher particle charge $\left(Z_{d} \propto T_{e}\right)$, and this increases the dust plasma frequency $\omega_{d}$. A reduced ratio $v_{d} / \omega_{d}$ and a shift of the stability boundary to higher values follows. Again, a higher critical pressure $p_{\text {cond }}$ follows. This contribution might be of minor importance due to the small change of $T_{e}$.

The role of $T_{e}$ can be more important for small discharge powers around $1 \mathrm{~W}$. If the electron density becomes small, the electron temperature rises to sustain the plasma. This effect can be enhanced by electron depletion by the dust component, what further increases $T_{e}$. As already mentioned, the situation at low discharge powers is expected to be complicated.

Finally, the ion streaming velocity has to be discussed. For higher discharge powers, the plasma potential relative to the electrode is higher. Thus, the ions gain more energy in the plasma-wall sheath, and the ion streaming velocity $u_{i}$ is higher. This increases the ion-neutral collision frequency $v_{i}$, which results in a more effective damping of the ion-two stream instability. Hence, the critical pressure becomes lower.

Additionally, the Mach number in equation (3.43) is larger for higher ion streaming velocities and this leads to a shift of the stability boundary to lower values. All together, an increase of $u_{i}$ leads to a reduced critical pressure $p_{\text {cond }}$. This is an opposite contribution to the other effects treated before, but in sum the observed increase can be explained by the theoretical predictions.

In figure 5.15, the product $n_{d} \cdot h_{c}$ is nearly the same for both particle sizes for discharge powers above $16 \mathrm{~W}$. Here, the impact of $n_{d} \cdot h_{c}$ is assumed to be equal, for the two particle sizes. Therefore, the gap between the curves of $p_{\text {cond }}$ in (a) can be related explicitly to the different particle sizes, considering that all other parameters are kept the same.

The theory of the ion-two stream instabilities can explain this gap between the curves, according to the dust particle radius. On the one hand, the particle charge number $Z_{d}$ is pro- 
(a)

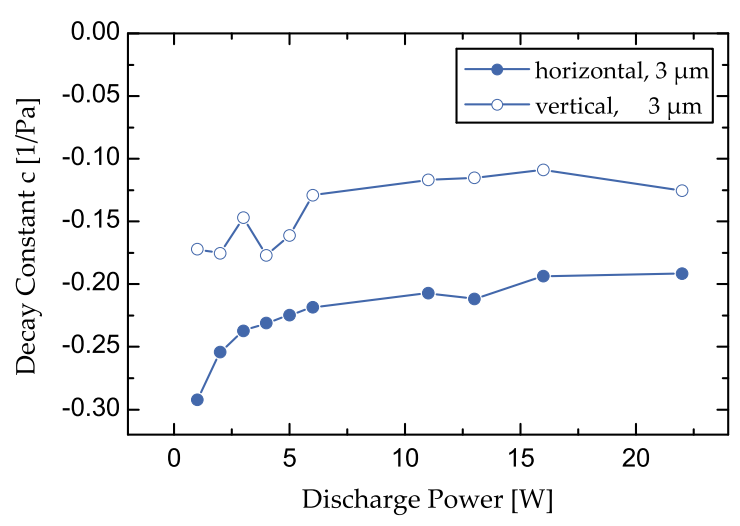

(b)

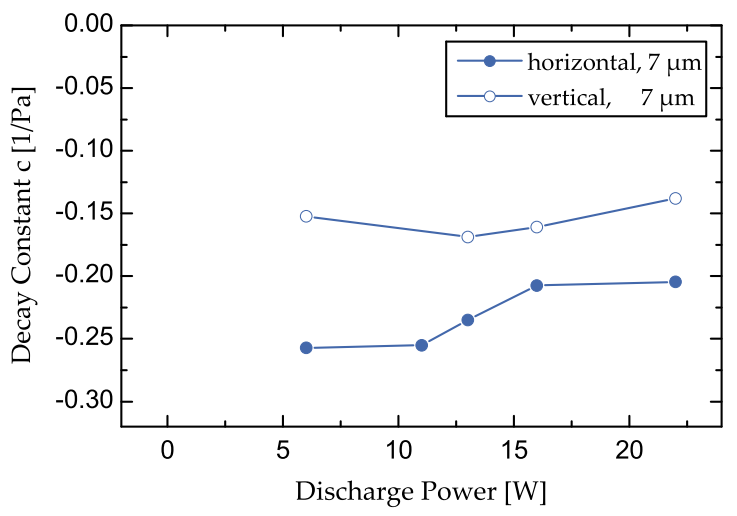

Figure 5.16: Decay constant $c$ of the exponential decay of the thermal energy in the melted state. (a) The decay constant for $3 \mu \mathrm{m}$ particles and (b) for $7 \mu \mathrm{m}$ particles. The horizontal component (closed symbols) and the vertical component (open symbols) of motion are shown.

portional to the particle radius $a$. Hence, the dust plasma frequency $\omega_{d}$ is larger for bigger particles. This shifts the stability boundary to higher values and decreases $v_{d} / \omega_{d}$. The critical pressure $p_{\text {cond }}$ is increased by this. On the other hand, the particle mass changes like $a^{3}$, which leads to a reduction of the plasma frequency $\omega_{d}$, with a stronger effect on $p_{c o n d}$ in the opposite direction. Altogether, the critical pressure for condensation will drop with an increase of the particle radius. This is confirmed by the experimental observations. The agreement between theory and experiments of two different particle sizes examined in independent measurements emphasizes the fundamental character of this model.

Figure 5.16 shows another fit parameter of the modified Boltzmann fit, the decay constant $c$ of the thermal energy. The decay constant $c$ is plotted versus the discharge power for (a) $3 \mu \mathrm{m}$ particles and (b) $7 \mu \mathrm{m}$ particles. In (b), the decay constant for powers below $6 \mathrm{~W}$ cannot be determined precisely due to statistical errors.

For both diameters, the decay constant is more negative for the horizontal component for all discharge powers. The difference between the components is nearly constant, in particular for the smaller particles. The more negative decay constant for the horizontal component represents a growing imbalance between the components, when the pressure is increased. This means, that in the highly energetic state the horizontal and vertical component are more equal than at the phase transition. The components tend to be in a more isotropic state. This is observed for both particle sizes.

The decay constant levels off for high discharge powers and drops at low powers similar to the curve of $p_{\text {cond }}$. As already noted, the plasma shows a quite different behavior at low powers.

Interestingly, the decay constant seems to be independent of the particle size and mass. For both particle sizes, the decay constant levels off at about $-0.13 \mathrm{~Pa}^{-1}$ for the vertical compo- 
nent and $-0.23 \mathrm{~Pa}^{-1}$ for the horizontal component. This is different compared to the observations at the critical pressure.

\subsubsection{Gradual Melting from the Bottom to the Top of the Dust Cloud}

This section studies a main feature of phase transitions in three-dimensional complex plasmas under gravity. The transition does not appear simultaneously in the whole system, but a transition front moves vertically through the dust cloud. The melting transition for example is initiated at the very bottom of the dust cloud and the transition front moves upwards until the whole cloud is in the disordered state (see figure 3.6).

The aim of the measurements presented here is to analyze the movement of energy fronts through the particle system for the vertical as well as for the horizontal component of motion. It is not clear beforehand, if the energy fronts move with the same speed for both components, or if they move independently. Independent movement of energy fronts might be possible for different heating mechanisms in both directions, for example caused by independent instability modes.

In contrast to the previous measurements, which study the condensation transition of the dust ensemble, the melting transition is examined in this section. As mentioned in the theory section about phase transitions in complex plasmas, melting and condensation are triggered by different types of instabilities. Nevertheless, the transition curve of the thermal energy $T_{e V}$ still can be described by the modified Boltzmann fit. This makes it possible to do the same analyses as for the condensation transition. The only difference to be expected is a certain shift of the critical pressure of the transition. This is discussed in detail in section 5.4

Two particle sizes of $3 \mu \mathrm{m}$ and $7 \mu \mathrm{m}$ are studied again. This gives insight into the role of particle size and mass on the observations and allows to estimate the significance of the results.

The experimental procedure is slightly different compared to the previous measurements. The confinement voltages were $40 \mathrm{~V}$ and the external bias voltage was $19 \mathrm{~V}$, which lies in the stable regime as described in section 5.3.2. The pressure was decreased from $25 \mathrm{~Pa}$ to $5 \mathrm{~Pa}$ for the $3 \mu \mathrm{m}$ particles and from $20 \mathrm{~Pa}$ to $4 \mathrm{~Pa}$ for the $7 \mu \mathrm{m}$ particles in steps of $1 \mathrm{~Pa}$. After a change of pressure, the system had $5 \mathrm{~min}$ to reach the new equilibrium. To realize the scan through the particle cloud, the whole discharge chamber was moved in vertical direction. A precise mechanical drive of the chamber, as described in the experimental section 4.1, makes it feasible to move the dust cloud relative to the scattering volume without disturbance of the particle system. The discharge chamber was moved downwards in steps of $0.5 \mathrm{~mm} \pm 0.1 \mathrm{~mm}$. One step is comparable to the diameter of the scattering volume. After a scan was finished, the chamber was moved to the start position and the next pressure was adjusted. After 5 min the next scan was started.

Depending on the extension of the cloud, up to 25 positions were probed for each pressure. 
(a)

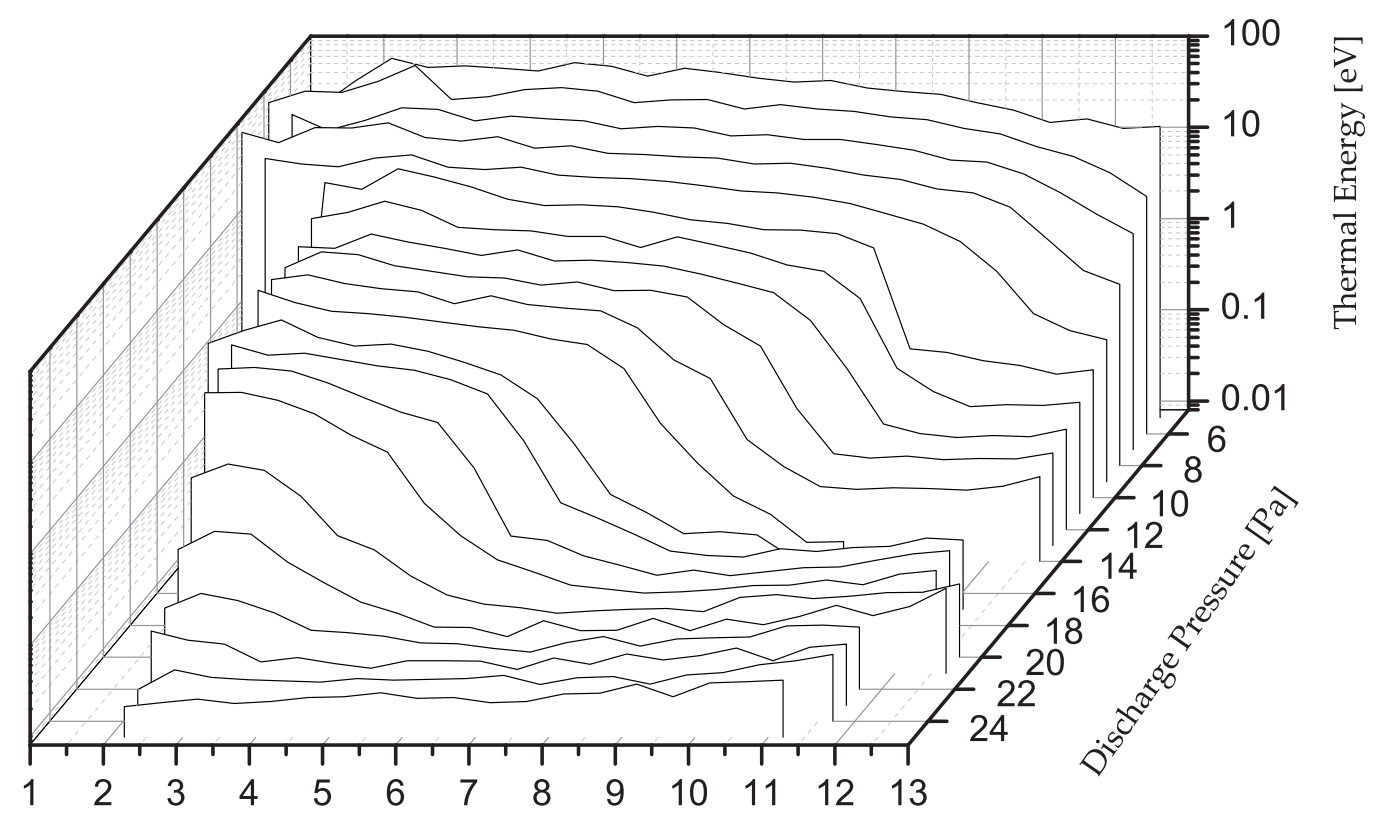

Height $[\mathrm{mm}]$

(b)

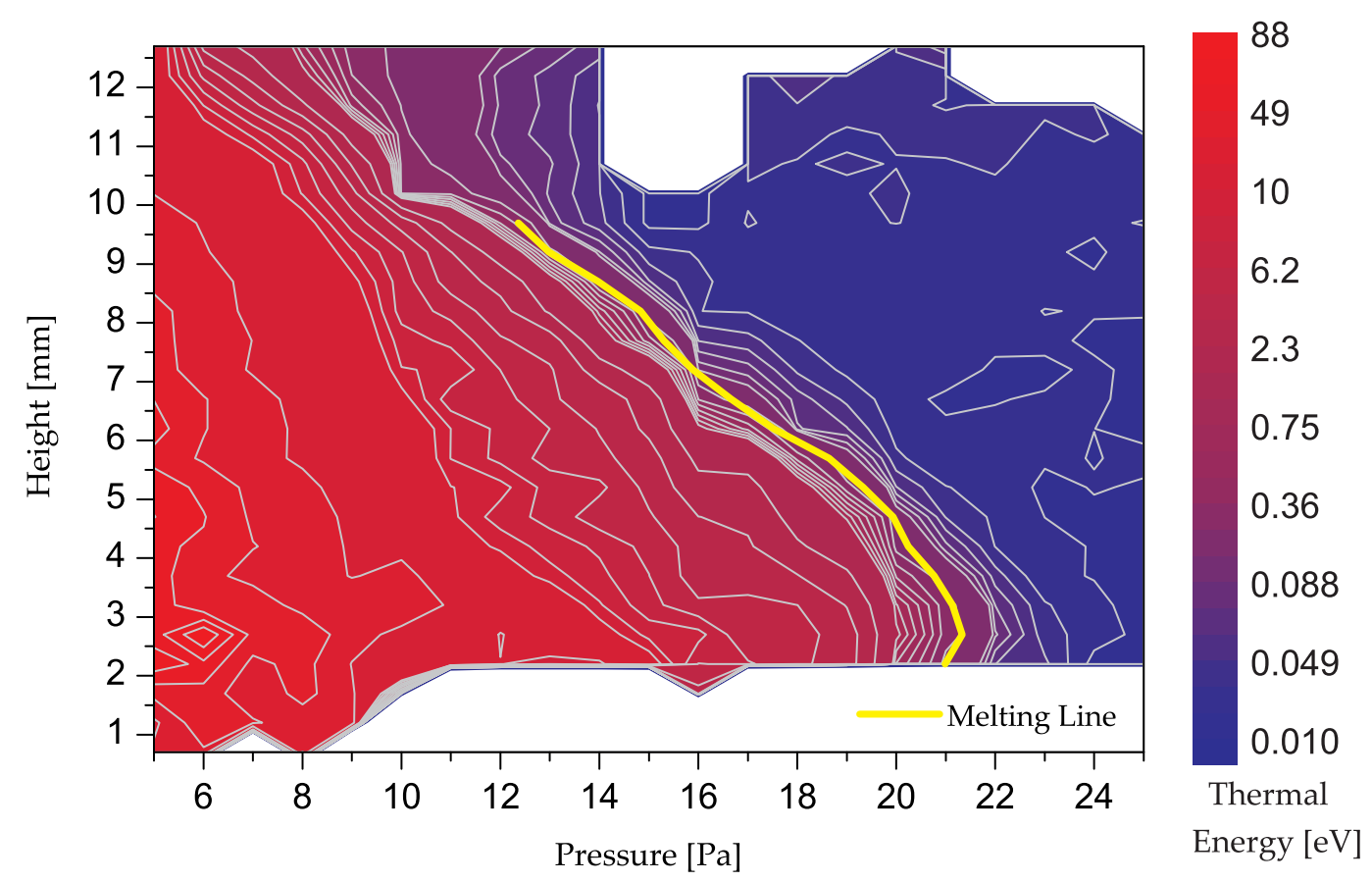

Figure 5.17: (a) Map of the thermal energy in the horizontal plane for different positions of the scattering volume above the lower electrode. The pressure was varied from $25 \mathrm{~Pa}$ to $5 \mathrm{~Pa}$. (b) Contour plot of the same data. The lower left corner of the graph corresponds to the rearward left corner of the upper graph. The energy is color coded. The thick line (yellow) marks the melting transition. 
So, up to 25 samples of the scattered intensity were taken and the corresponding autocorrelation functions are calculated. In sum, this gives for all pressures, both components of motion and for two particle sizes about 1800 autocorrelation functions to fit with a Gaussian function. The fitting of such a huge set of autocorrelation functions is done automatically by a C-routine as described in section 4.3.3.

The result of the scanning procedure is illustrated in figure 5.17 (a). Here, the mapping of the thermal energy $T_{e V}$ for a melting transition of a dust ensemble consisting of $3 \mu \mathrm{m}$ particles is presented. This figure relates to the movement in the horizontal plane. The $x$-axis refers to the position of the scattering volume relative to the lower electrode and the y-axis refers to the discharge pressure. The thermal energy $T_{e V}$ is low all over the dust cloud at high pressures. The system is in the uniform, ordered phase state. At mediate pressures, the lower part of the dust cloud is melted with energies of the order of $10 \mathrm{eV}$, and the upper part is still in the ordered state. At low pressures, the thermal energy is distributed nearly uniform again, the system is in the disordered state. The height of the dust cloud changes from $9 \mathrm{~mm}$ at $25 \mathrm{~Pa}$ to $11.5 \mathrm{~mm}$ at $5 \mathrm{~Pa}$ during the melting transition.

Figure 5.17 (b) depicts a contour plot of the data in (a). The lower left corner of (b) corresponds to the rearward left corner of (a). The ordinate denotes the vertical coordinate in the dust cloud and the abscissa is the discharge pressure. The thermal energy is color coded. The contour lines seem to be straight lines. This means, that the melting transition moves with constant progress from the bottom to the top. The melting transition is marked by a thick (yellow) line. It represents the energies at the melting pressure and is obtained from an analysis using the modified Boltzmann fit. The results from the modified Boltzmann fits are discussed at the end of this section.

This measurement demonstrates how DLS can be used for mapping of the thermal energy in the dust cloud during a phase transition. The corresponding plot for the vertical component of movement is displayed in the appendix, figure A.3. The phenomenon of gradual melting in a ground based complex plasma experiment has never been studied with this effort and accuracy before.

To study the heat transport in the vertical direction, a deeper analysis of the contour lines (as in figure 5.17) is done. The contour lines are extracted and fitted by a linear function. Some of the contour lines need to be cropped at the bottom, because boundary effects lead to deviations from linearity. The slope of the contour line of a certain energy describes the distance the energy front moves upwards for a change of the discharge pressure. In a totally uniform system, the melting would appear at all heights at the same time and the contour lines would be vertical.

The results of this procedure are discussed in two parts. Firstly, the lower energy range around the melting energies are treated, and secondly the high energy state is discussed.

Figure 5.18 depicts the slopes of the linear contour lines for low energies for (a) $3 \mu \mathrm{m}$ particles and (b) for $7 \mu \mathrm{m}$ particles. The horizontal (squares) and the vertical component (circles) 
(a)

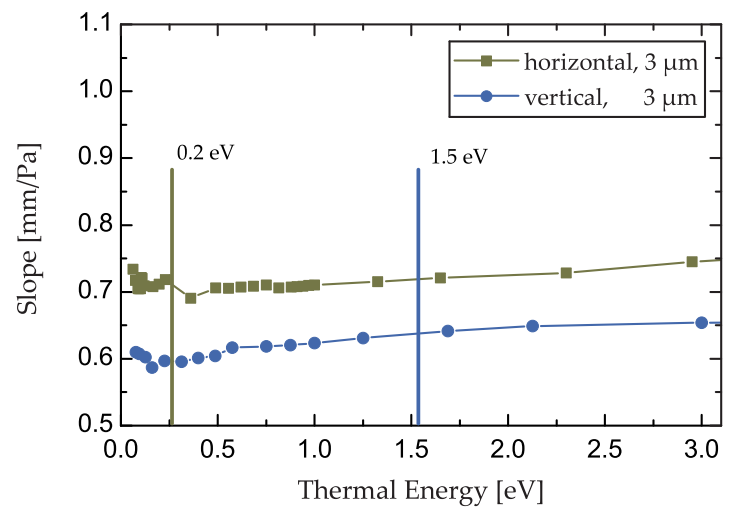

(b)

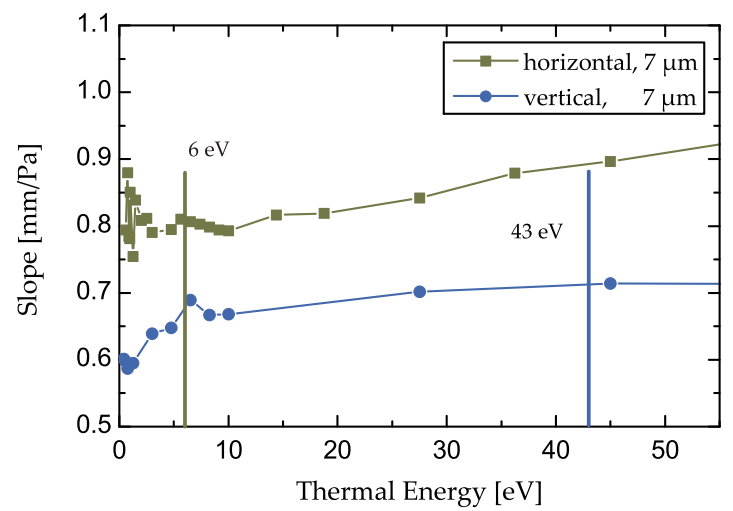

Figure 5.18: Slopes of the contour lines for energy values near the melting transition. Figure (a) displays the horizontal (squares) and vertical (circles) component of $3 \mu \mathrm{m}$ particles and (b) shows the two components for $7 \mu \mathrm{m}$ particles. A similar behavior is observed for both particle sizes. The energy transport is slightly faster for the horizontal component and nearly constant for all energies.

of movement are plotted. The $3 \mu \mathrm{m}$ particles in (a) have a slope of about $0.7 \mathrm{~mm} / \mathrm{Pa}$ for the horizontal movement and about $0.6 \mathrm{~mm} / \mathrm{Pa}$ for the vertical component at low energies below $0.5 \mathrm{eV}$. The curves have a slight increase of about $0.05 \mathrm{~mm} / \mathrm{Pa}$ toward $3.0 \mathrm{eV}$. That means, higher energies are transported slightly faster through the dust cloud. A significant observation is the gap between the slopes for the horizontal and vertical component. The energy of movement in the horizontal component is transported faster by $0.1 \mathrm{~mm} / \mathrm{Pa}$ compared to the vertical component.

It has to be noted that the two components are not necessarily in the same phase state at same thermal energies. The energies corresponding to the critical pressure $T_{\text {melt }}$ are indicated in figure 5.18 by vertical lines. The line at $0.2 \mathrm{eV}$ corresponds to the horizontal component and the one at $1.5 \mathrm{eV}$ is related to the vertical component.

A very similar result is obtained from a measurement on $7 \mu \mathrm{m}$ particles, as illustrated in figure 5.18 (b). The horizontal component has a slope of about $0.8 \mathrm{~mm} / \mathrm{Pa}$, where the vertical component has a slope of about $0.65 \mathrm{~mm} / \mathrm{Pa}$ at low energies. The increase toward higher energies is a little stronger than for the $3 \mu \mathrm{m}$ particles. The horizontal component again transports the energy faster than the vertical component. The difference between the slopes is about $0.15 \mathrm{~mm} / \mathrm{Pa}$, and therefore slightly bigger than for the $3 \mu \mathrm{m}$ particles. The melting transition energies are $6 \mathrm{eV}$ for the horizontal component and $43 \mathrm{eV}$ for the vertical component. The observed thermal energies for the $7 \mu \mathrm{m}$ particles are of an order of magnitude larger than for the $3 \mu \mathrm{m}$ particles. These results indicate that the particle size and mass do not have a strong influence on the movement of the energy fronts through the dust cloud.

The different energy transport of the horizontal and vertical component can be explained by different instability modes. As discussed in section 3.3.2, the ion-two stream instability 
(a)

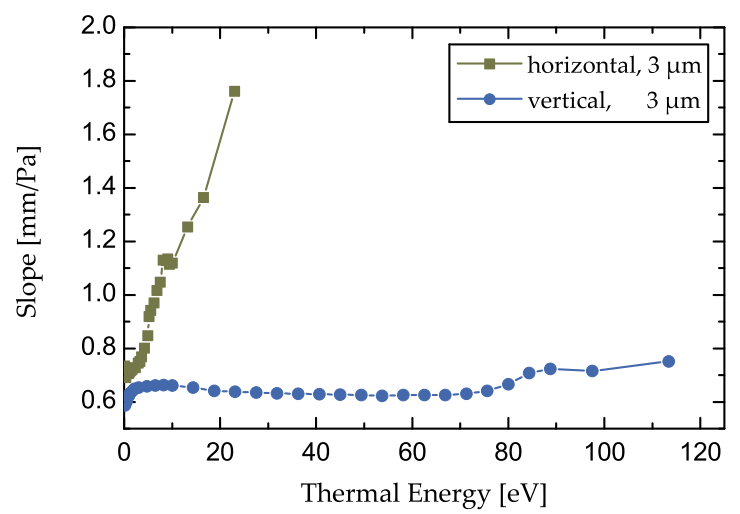

(b)

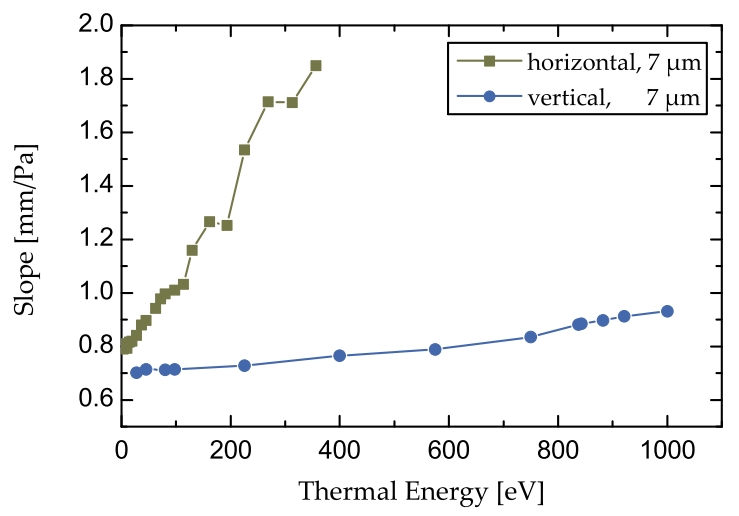

Figure 5.19: Slopes of the contour lines for energy values above the melting transition. The horizontal and vertical component are shown for (a) $3 \mu \mathrm{m}$ particles and for (b) $7 \mu \mathrm{m}$ particles. For both particle sizes a faster movement of energy fronts is seen for the horizontal component (squares) and the movement gets faster for higher energies. On the other hand, the energy transport is nearly constant for the vertical component (circles).

is expected to be responsible for the anomalous heating in the melted state. As the authors have demonstrated in [7, 8, 9], not only a single mode can be present, but various modes can appear perpendicular and parallel to the ion flow. This can lead to different heating in the horizontal and vertical direction. The change of plasma parameters like Mach number, plasma density etc. can have different consequences on the growth rate of the instabilities. To prove this explanation, further simulations and experiments are necessary.

The second part of this analysis focuses on higher particle energies. Figure 5.19 illustrates the slopes of the contour lines above the melting energy. Again, the results for both components of movement are plotted for (a) $3 \mu \mathrm{m}$ particles and (b) $7 \mu \mathrm{m}$ particles.

The measurements yielded qualitatively the same results for the two particle sizes, again. The horizontal component (squares) reveals a very different behavior compared to the vertical component (circles). The energy transport for the vertical movement is mainly constant for all energies, whereas the horizontal component reveals a strong increase of the slope of contour lines for higher energies. In the case of $7 \mu \mathrm{m}$ particles, the slopes of the vertical component slightly increases for higher energies and the slopes of the horizontal rises less compared to the $3 \mu \mathrm{m}$ particles.

The coupling between the particles is low in the high energetic state at low discharge pressures, and the system acts like a non-ideal gas. The exchange of energy is enhanced by the free particle movement over long distances. Therefore, a more uniform system can be expected and the slopes of the contour lines should increase. This is observed for the energy transport of the horizontal component. In contradiction, the slopes of the vertical particle motion stay mainly constant. The system becomes strongly anisotropic, with a horizontal component that shows enhanced energy transport and a suppressed energy transport for the vertical particle 
(a)

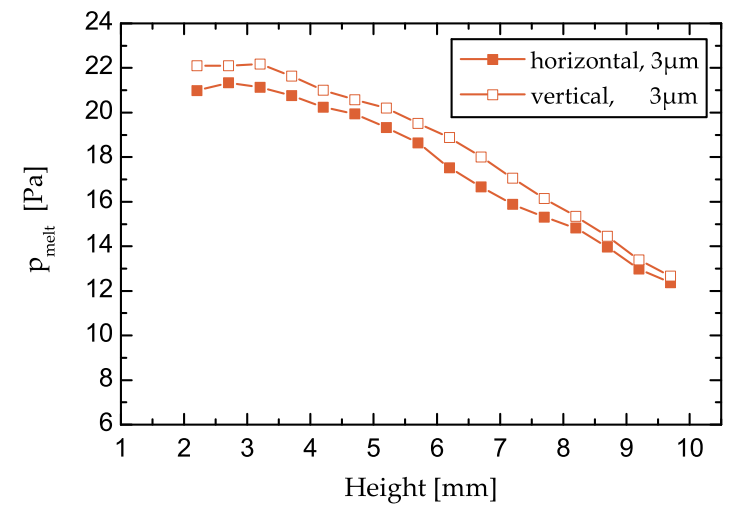

(b)

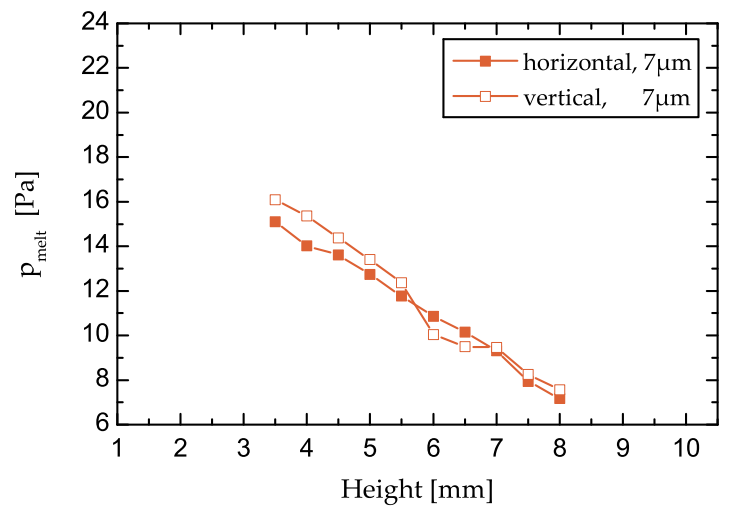

Figure 5.20: The critical pressure for the melting transition $p_{\text {melt }}$ is shown for (a) $3 \mu \mathrm{m}$ and (b) $7 \mu \mathrm{m}$ particles for the horizontal (closed symbols) and the vertical (open symbols) component of motion. The melting pressure drops with the height, i.e. melting starts at the bottom of the dust cloud. In (a), slight deviations from linearity are due to nonlinearities in the sheath electric field in the vicinity of the lower electrode.

movement.

Another analysis of the data in figure 5.17 (a) is done by applying the modified Boltzmann fit to the phase transition curves of the thermal energy for different heights. Figure 5.20 depicts the critical pressure of melting for the (a) $3 \mu \mathrm{m}$ and (b) $7 \mu \mathrm{m}$ particles for both components of motion. As expected, the melting pressure is lower at the top of the dust cloud and a nearly linear decay is found. There are deviations from linearity near the lower electrode in (a). This might be due to nonlinearities in the sheath electric field close to the electrode. This effect is not observed for $7 \mu \mathrm{m}$ particles in figure (b), because bigger particles were not trapped in the vicinity of the lower electrode.

In consistency with earlier observations, the critical pressure is higher for the vertical than for the horizontal component. The curves indicate that the gap between the components is vanishing at the top of the dust cloud. This is what can be expected regarding to the discussion above. The melting transition for the vertical component starts at higher pressures than the horizontal component. This means, the melting front of the vertical component is ahead the melting front of the horizontal component. Considering that the melting transition front moves faster for the horizontal component (see figure 5.18), the critical pressures come closer with increased height. In the end, the critical pressure for the horizontal component is larger than the one for the vertical component. This situation is not observed in this work, where dust clouds with heights of less than $10 \mathrm{~mm}$ are studied.

Figure 5.21 displays the decay constant $c$ of the thermal energy at low discharge pressures of $3 \mu \mathrm{m}$ particles. Both components of movement are displayed in (a) and (b) shows the ratio of the decay constants of horizontal and vertical component of motion. The constant $c$ describes the growth rate of energy toward lower discharge pressures for a melting transition. 
(a)

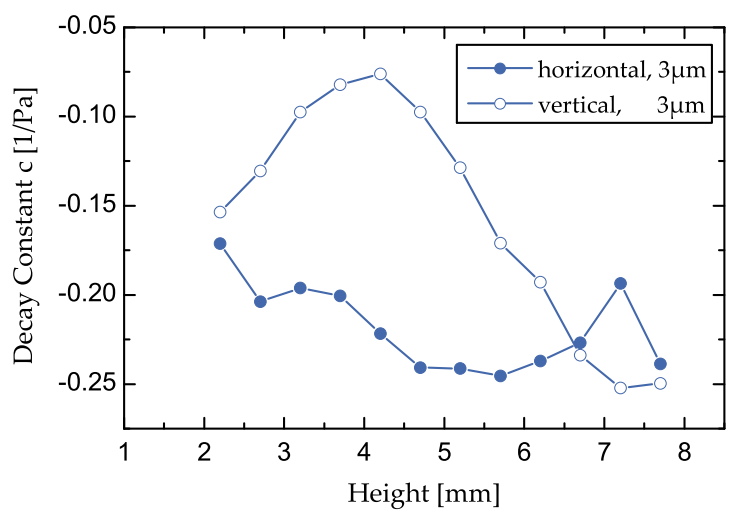

(b)

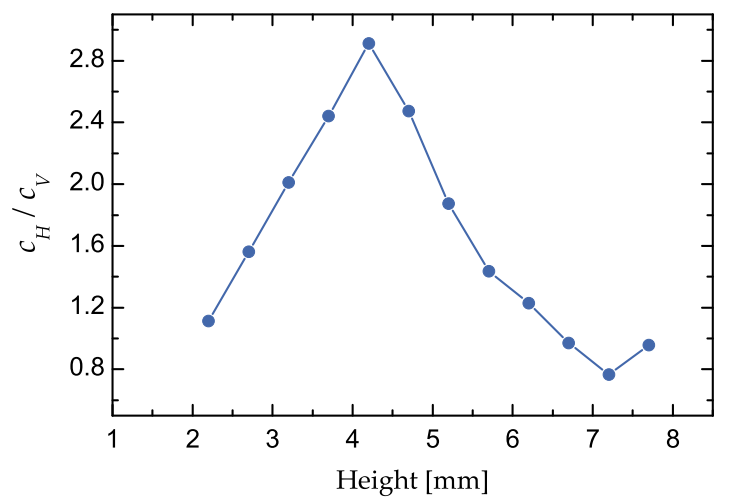

Figure 5.21: (a) Decay constant for horizontal $c_{H}$ (closed symbols) and vertical $c_{V}$ (open symbols) components of movement for $3 \mu \mathrm{m}$ particles. Figure (b) depicts the ratio of the decay constant of both components. The decay constant (growth rate of energy) $c$ is about three times larger in the horizontal plane than in the vertical in the center of the dust cloud.

The vertical component is found to have a reduced growth rate in the center of the dust cloud. The growth rate of energy for the horizontal component is about three times larger than for the vertical component in the center of the dust cloud. Thus, the energies in horizontal and vertical direction are equalized more effectively in the center than in the outer parts.

The analysis shown here examine the behavior of the two components during the gradual melting transition. The unique experimental approach gives insight into the impact of different types of interaction potentials in the horizontal and vertical direction on the melting transition. No previous works exist to the present day that studies the movement of heating fronts in a ground based complex plasmas in such detail as done here.

\subsubsection{Anisotropy between Horizontal and Vertical Movement}

The anisotropy of the horizontal and vertical particle motion was already mentioned earlier. It results from the anisotropy in the plasma environment. The sheath electric field perpendicular to the electrode leads to enhanced heating in vertical direction in the melted state. The thermal energy of the vertical component exceeds the energy in the horizontal plane by a factor of about 10. The energies are equalized in the ordered state, because the source of anomalous heating, the ion-two stream instability, is damped.

In this section, the data of the previous measurements are analyzed according to the anisotropy of the thermal energy. The ratio of the thermal energy in horizontal and vertical direction $T_{H} / T_{V}$ is examined under variation of plasma parameters that are discussed in the previous sections.

Figure 5.22 illustrates the ratio $T_{H} / T_{V}$ during the condensation transition for a variation 
Figure 5.22: The ratio of the thermal energy in horizontal and vertical direction $T_{H} / T_{V}$ during the condensation transition for discharge powers between $1 \mathrm{~W}$ and $22 \mathrm{~W}$ is illustrated. The ratio shows to be independent of the power for pressures between $10 \mathrm{~Pa}$ and $14 \mathrm{~Pa}$. For higher pressures, the system tends to the ordered state with equalized thermal energies. At low pressures, a bigger spread of the data is caused by dust acoustic waves.

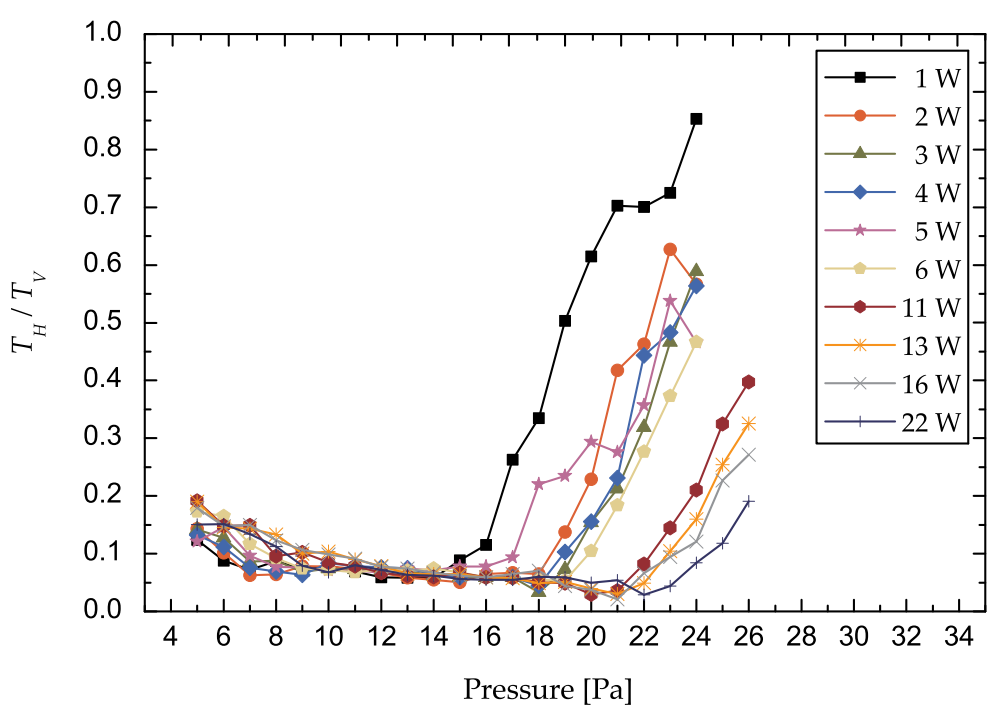

of the discharge power between $1 \mathrm{~W}$ and $22 \mathrm{~W}$. This measurement is discussed in section 5.3.4. The plot reveals a remarkable feature of the anisotropy. The ratio $T_{H} / T_{V}$ is independent of the discharge power between pressures of $10 \mathrm{~Pa}$ and $14 \mathrm{~Pa}$, so just before the condensing transition.

The ratios are still comparable at lower pressures, but the deviations are stronger. These deviations can be explained by the cooling effect observed for the vertical component when the particles reach the vicinity of the lower electrode. The cooling is most effective, when dust acoustic waves are present (see section 5.3.2). The excitation and amplitude of dust acoustic waves depend on the discharge pressure and power, hence the ratios $T_{H} / T_{V}$ show different deviations for the applied powers.

The condensation transitions can be seen at higher pressures. Here, the ratio of energies strongly rises and tends to equality. The thermal energy of vertical motion drops faster than the horizontal energy until they are equal. As discussed the critical pressure of condensation depends on the discharge power. That is why the curves do not superimpose at higher pressures. This result is compared to other parameters that are studied so far. A similar analysis as illustrated in figure 5.22 is performed for the external bias voltage, the confinement voltage, the particle density, the particle size, and the variation of the vertical position of the scattering volume. For all these parameters, the ratio $T_{H} / T_{V}$ is seen to be constant just below the critical pressure of condensation.

To compare all these results, an average curve of $T_{H} / T_{V}$ was calculated for the sequences of each parameter variation. Only the data points below the critical pressure of condensation were considered for averaging. So, the data of the power variation in figure 5.22 for example, are reduced to a single average curve. Figure 5.23 depicts the averaged curves for all parameters listed above. The power variation (figure 5.22) is represented by upside down triangles. The errors displayed here are calculated by Gaussian error propagation assuming an error for 


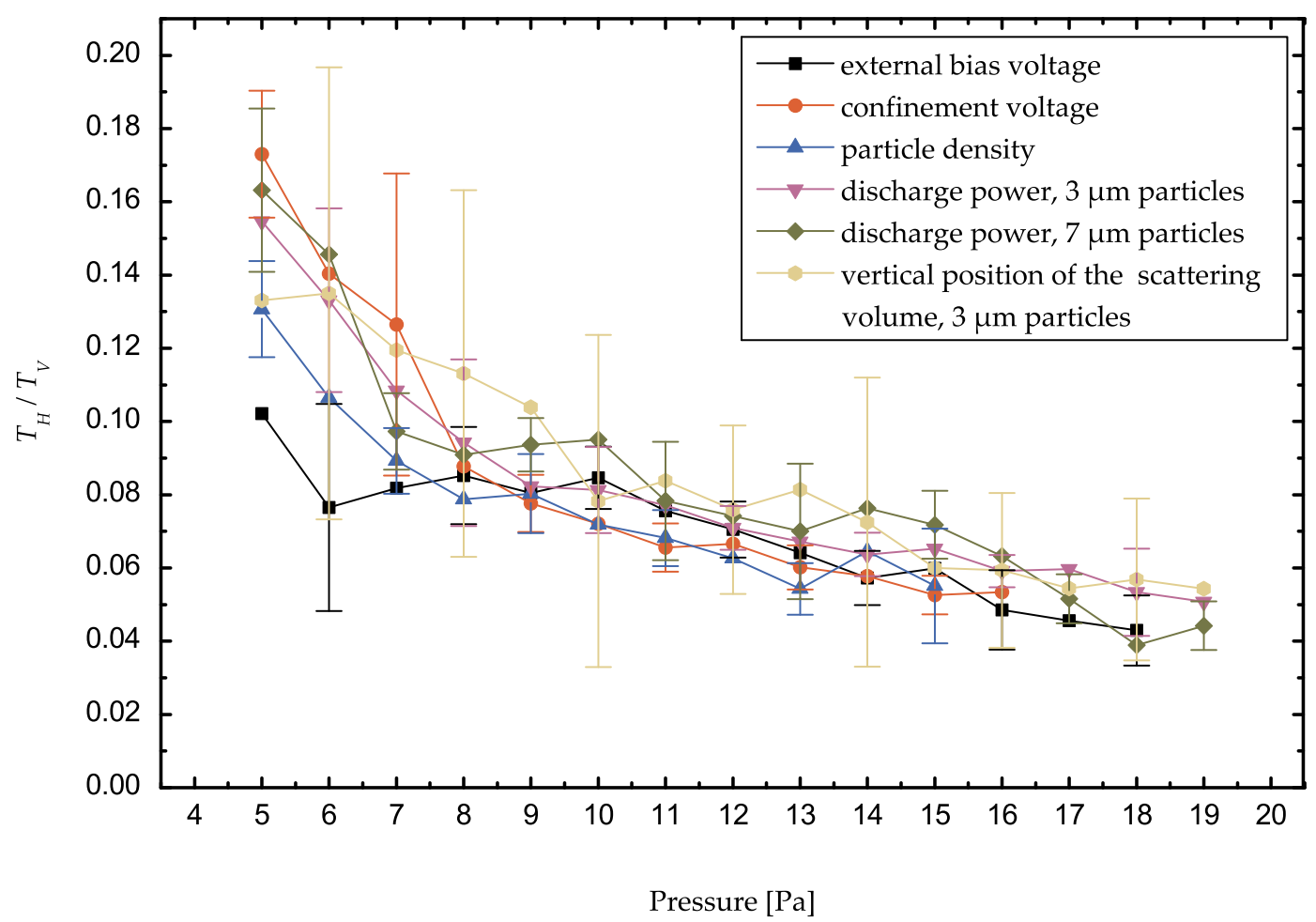

Figure 5.23: The average ratios $T_{H} / T_{V}$ of the sequences of each parameter variation. Only the points for pressure below the critical pressure of the phase transition are considered for the averages. The average ratios for external bias voltage, confinement voltage, particle number density, discharge power and particle size, and vertical position of the scattering volume are shown. The average ratios are independent (within error bars) of the experimental parameters studied.

the thermal energy of $20 \%$ for the horizontal component and $10 \%$ for the vertical component (see section 5.2). The standartd deviations of the caculated averages are taken for the error bars at low discharge pressures, because it exceeds the error from error propagation.

The ratio $T_{H} / T_{V}$ shows nearly no dependency on any experimental parameter studied here. The calculated averages have overlapping error bars and are less than 0.04 apart for pressures between $8 \mathrm{~Pa}$ and $19 \mathrm{~Pa}$. The thermal energy in vertical direction is between 10 and 25 times larger than the horizontal energy in this range. The ratio $T_{H} / T_{V}$ generally rises for a decrease of discharge pressures. That is, the system gets more isotropic. At low pressures, the results are affected by the cooling of the vertical component through dust acoustic waves, as described in section 5.3.2. This leads to a larger standard deviation of $T_{H} / T_{V}$.

The independence of $T_{H} / T_{V}$ on various plasma parameters makes it a universal property for three-dimensional complex plasmas under gravity. In particular, since the change of the particle size, the vertical position of the measurement and the discharge power have no impact on the ratio $T_{H} / T_{V}$. These are significant parameters that determine the structure and dynamics of the system.

These observations suggest that the amplitudes and growth rates of the ion-two stream 


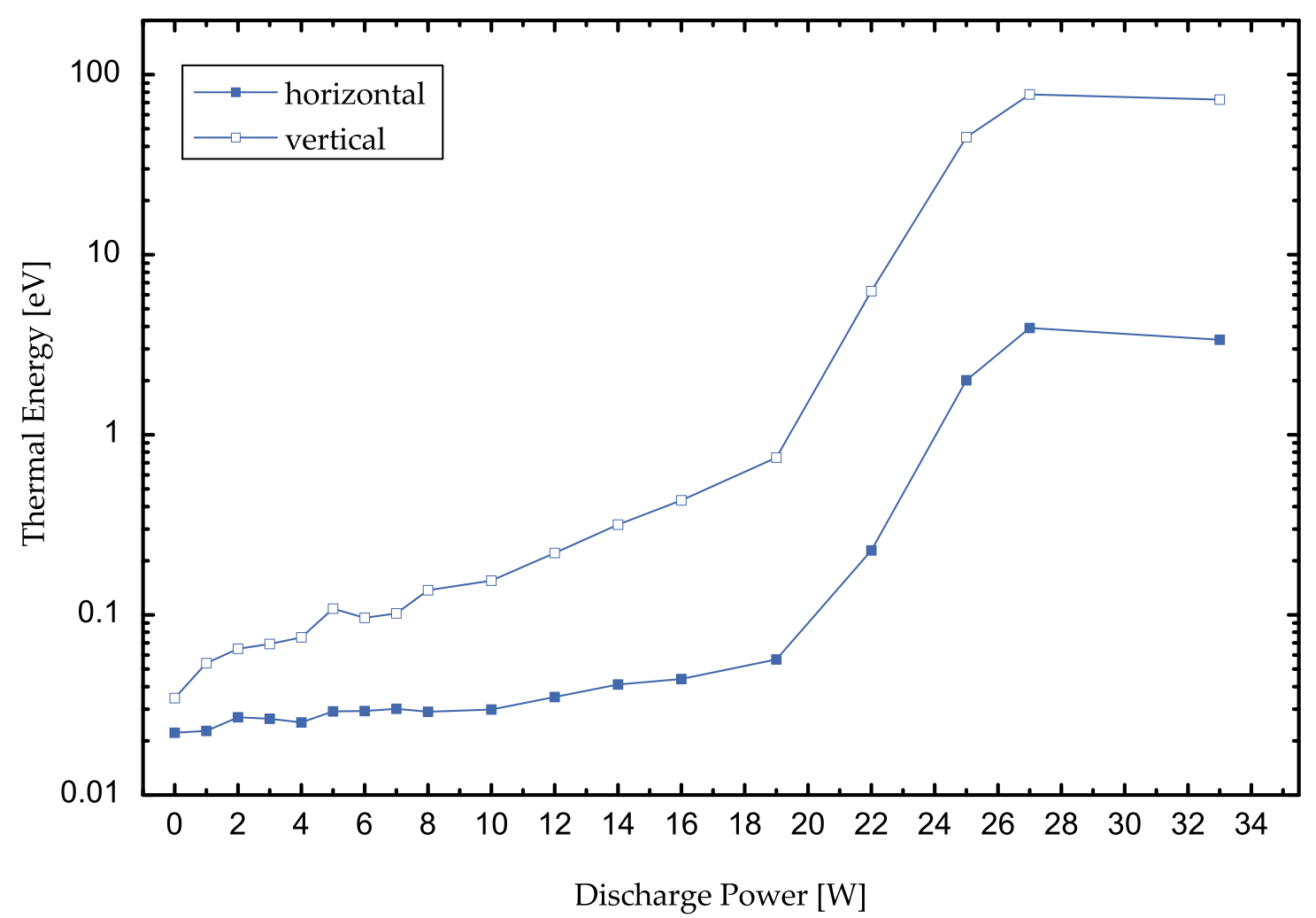

Figure 5.24: The thermal energy for the horizontal (closed symbols) and vertical (open symbols) component of particle motion during a melting transition induced by a rise of the discharge power. A two step melting process is observed. First, the energies grow exponentially until a discharge power of $19 \mathrm{~W}$ is reached. A second and stronger increase is seen above $19 \mathrm{~W}$, which saturates for powers above $27 \mathrm{~W}$.

instability modes in horizontal and vertical direction have the same dependency on external plasma parameters. Furthermore, the heat transfer between the horizontal and vertical component of motion through dust-dust collisions has to be constant for all plasma parameters or has to be of minor importance. In the latter case, the ratio $T_{H} / T_{V}$ does not change significantly due to the coupling between the two components. To prove these assumptions and to get a deeper understanding of these observations, further simulations and subsequent experiments are necessary.

The constancy of $T_{H} / T_{V}$ is a remarkable property of the disordered state and has never been reported so far, whether in theory, nor in experiments. The examination of vertical and horizontal component is difficult in most complex plasma experiments, and the detailed analysis of the instability modes, that are responsible for heating of each component, and the coupling between the two components is still an open issue. 


\subsubsection{Melting Induced by the Increase of the Discharge Power}

The phase transitions can also be induced by variation of the discharge power for a fixed pressure. The change of the discharge power has much stronger consequences for the whole plasma state than changing the pressure. The phase transition is therefore expected to look different. Compared to the variation of the pressure, where mainly the friction rate changes, the variation of the discharge power is a more complex way to cause a phases transition.

Here, the melting transition induced by the increase of the discharge power is presented briefly. Figure 5.24 shows the thermal energies of the two components of motion during the melting transition. The transition curves reveal a different form compared to the transition induced by a variation of the pressure. The modified Boltzmann fit does not give an appropriate model, but a two stage melting process is observed.

At the initial conditions below $1 \mathrm{~W}$, the system is in a fluid like state close to the highly ordered crystal state. The vertical component has already slightly higher energy than the horizontal component. For an increase of the discharge power up to $19 \mathrm{~W}$, a nearly exponential increase of the thermal energy is observed for both components with a larger growth rate for the vertical component. Between $19 \mathrm{~W}$ and $27 \mathrm{~W}$, a second much stronger growth of the energy is observed. Then the energies saturate for discharge powers above $27 \mathrm{~W}$. This is different to the melting by pressure variations, where a further exponential increase of the energy is observed in the melted state.

Several plasma parameters change significantly due to the variation of the discharge power. The plasma density and temperature are affected and this leads to a change of the plasma frequency and Debye length. The position of the sheath edge, the width of the sheath and electric field in the sheath and therefore the ion streaming velocity are modified by a change of discharge power. This affects the crystalline structure and the damping of the instability responsible for the phase transitions.

\subsubsection{Summary}

In this section, DLS is applied to investigate the phase transitions between the melted state and the fluid-like state of a complex plasma under gravity. The thermal energy of the dust component is used as the characteristic quantity to describe the phase transitions. The transitions are caused by reduction of the discharge pressure and are studied under variation of the most fundamental experimental parameters.

The thermal energy of the dust particles reveals a characteristic curve during the phase transition. A single-step transition is observed in a similar form for the melting as well as for the condensing transition. Below the critical pressure of the phase transition, the thermal energy increases exponentially with a decreases of the pressure.

The application of an external bias voltage to the upper electrode shows to have impact on the phase transitions. The critical pressure and the energy level that is reached in the melted 
state are affected. A stable range of voltages, in which the impact on the phase transition is reduced, is found for positive and for negative voltages. The confinement voltages have only little impact on the phase transitions and can be chosen freely.

The vertical component of motion loses energy in the vicinity of the lower electrode in the melted phase state. The dust particles can come close to the lower electrode due to large amplitude dust acoustic waves. The losses can be connected to the nonlinearities in the sheath electric field near the electrode. The horizontal component of motion is not affected by this kind of energy dissipation.

The particle number density and height of the dust cloud turn out to be critical parameters for the phase transition. The impact can be reduced by ensuring sufficiently large densities and heights.

As a main plasma parameter, the discharge power is examined for two different particle sizes. The phase transitions show the same qualitative dependency on the discharge power for both particle sizes. The phase transitions of bigger dust particles are shifted to lower discharge pressures. The impact of the discharge power is mainly due to changes in the particle number density and the height of the dust cloud.

The theory of ion-two stream instabilities makes predictions according to the impact of a change of the main plasma parameters on the phase transitions (see section 3.3.2). The observations in the experiments are in good agreement with the predictions of the theory.

The gradual melting transition from the bottom to the top of the dust cloud is studied by scanning vertically through the dust cloud at different discharge pressures. The evolution of the energy distribution in vertical direction is measured during the melting transition and the movement of energy fronts in vertical direction is examined. Measurements on two different particle sizes reveal qualitatively the same results. The energy fronts with energies comparable to the melting energy show nearly the same progress upwards for a change of pressure. Here, the energy fronts of the horizontal component move slightly faster than the energy fronts of the vertical component of movement.

At high particle energies, the movement of energy fronts of the horizontal component is much faster compared to the vertical component. Thus, the energy of the horizontal component of motion is distributed faster over the dust cloud than for the vertical component.

Furthermore, the growth rate of energy for a change of pressure in the melted state is studied. In the center of the dust cloud, it is much higher for the horizontal component than for the vertical component. At the top and the bottom, however, it is equal for both components. Thus, the dust system is strongly inhomogeneous with a spatial complex distribution of energy in the melted state.

The ratio of the thermal energy in horizontal and vertical direction $T_{H} / T_{V}$ shows to be constant just below the critical pressure of the phase transition. This is observed for all variations of plasma parameters and seems to be a universal property of strongly coupled complex plasmas. The ratio $T_{H} / T_{V}$ increases with lower pressures, and the system tends to isotropy in the 
highly energetic state.

Another way to melt the dust structure is studied by increasing the discharge power. The observed phase transition is of a different type. A two-step transition is observed with different characteristics compared to the phase transitions induced by reduction of the discharge pressure.

\subsection{Hysteresis Loop}

A remarkable result of the simulations of instability driven phase transitions is the prediction of a hysteresis loop [7, 8, 9]. The condensation transition is triggered by the ion-two stream instability and the melting is determined by the phonon stream instability. The damping of the two instabilities occurs at different discharge pressures, and therefore a hysteresis loop is observed. This is discussed in more detail in section 3.3.2. This section presents a high resolution DLS measurement to study the hysteresis loop of the phase transitions between fluid and gaseous state of a complex plasma.

The experimental observation of the hysteresis loop turns out to be difficult. The measurements of the transition curves of the thermal energy $T_{e V}$ as discussed in section 5.3 are based on determination of $T_{e V}$ in discrete steps. This is, a certain pressure is adjusted, the system has some minutes to reach equilibrium, and then the photomultiplier signals are attained and saved within several minutes. After this, the next pressure is adjusted and the acquisition procedure starts again. A sequence of a certain number of pressures can be examined automatically by this procedure. The observation of the hysteresis loop by such a discrete measurement was not successful. The data points near the transition pressure for the melting curve and the condensing curve showed overlapping error bars.

It follows, the hysteresis effect in a complex plasma experiment is much smaller than the simulation predicts. The simulations show a difference between the critical pressure of condensation $p_{\text {cond }}$ and melting $p_{\text {melt }}$ of the order of $17 \mathrm{~Pa}$. The disagreement to the experiment might be due to the different plasma conditions in the simulations. There, the situation is simplified by considering a uniform dust cloud that is unbounded in all directions. Furthermore, an infinite dust cloud is simulated using periodic boundary conditions [8]. In the experiment, the dust cloud is only several layers thick. The critical pressure of condensation is therefore lower, inasmuch as the ion-two stream instability is convective.

The study of a much closer hysteresis loop requires the experimental approach to be varied. The range of pressures close to the phase transition is bistable and the system can switch between both transition curves. Thus, it should be avoided to rest too long at a certain pressure and the discrete acquisition method is obsolete. A continuous and fast variation of the discharge pressure and simultaneous data acquisition is necessary instead.

For the continuous measurement, the discharge pressure was changed manually from 16 
$\mathrm{Pa}$ to $26 \mathrm{~Pa}$ to cause a condensing transition, and after that, the pressure was decreased again to induce the melting transition. The data acquisition time of the whole measurement was $67 \mathrm{~s}$, which is limited by the memory of the data acquisition hardware. The sample consist of $2 \cdot 10^{8}$ points measured with a frequency of $3 \mathrm{MHz}$. Only one component of motion could be measured at a time, because the second channel of the data acquisition card was used to monitor the discharge pressure. To study the horizontal and vertical component of motion, two independent experiments were performed.

The data sample of the continuous measurement is divided into parts to calculate the autocorrelation functions. Each part consists of a window of $2 \cdot 10^{6}$ points. After the autocorrelation function is calculated, the window is shifted by $1 \cdot 10^{6}$ points and the next calculation is initiated. Thus, the parts are overlapping to increase the resolution of the analysis. This results in about 200 autocorrelation functions and a temporal resolution of 0.33 s. Note, the pressure meter has a repetition time of $0.02 \mathrm{~s}$. The further analysis of the 200 autocorrelation functions is done by automatic fitting again, as described in section 4.3.1.

The DLS measurements presented in this section are done with scattering angles of about $7^{\circ}$. The other parameters of the DLS experiment are not different to the description in the experimental section 4.3.1. The discharge power was $2 \mathrm{~W}$ and the confinement voltage was $40 \mathrm{~V}$. The external bias voltage was set to $20 \mathrm{~V}$, which relates to the stable range as discussed in section 5.3.2.

Figure 5.25 shows the condensing and melting transitions for the horizontal (lower curves) and vertical (upper curves) component of motion. The critical pressure of melting is lower compared to the critical pressure of condensation for both components of motion. Thus, in both experiments the predicted hysteresis loop is observed. The error of the thermal energy $T_{e V}$ was estimated to be $\pm 15 \%$ after section 5.2

The observed hysteresis loop is much smaller than the prediction of the simulations. The critical pressure of the transitions is obtained by applying the modified Boltzmann fit. The critical pressure of condensation for the horizontal component was determined to be $p_{\text {cond }}=$ 19.7 Pa. The critical pressure for melting is $p_{\text {melt }}=19.1 \mathrm{~Pa}$. The analysis for the vertical component gives $p_{\text {cond }}=20.7 \mathrm{~Pa}$ and $p_{\text {melt }}=19.9 \mathrm{~Pa}$. Thus, the width of the hysteresis is only of the order of $0.8 \mathrm{~Pa}$, but the data points are separated without overlapping error bars. The energies differ up to a factor of 3 near the phase transitions.

Only a close range around the critical pressures of the transitions is examined, due to the limitation of the sample size by the hardware. Hence, only the lower part of the dust cloud (around the scattering volume) was in the melted state at start conditions of $16 \mathrm{~Pa}$. The upper part of the dust cloud remained in the ordered phase state during the measurements. This was used to estimate the particle number density during the phase transitions using the CCD method. The height of the dust cloud was estimated too. Both quantities were nearly constant during the measurements. The product of density and height $n_{d} \cdot h_{c}$ was of the order of 900 . This indicates a reduced impact of density and height on the phase transitions according to 


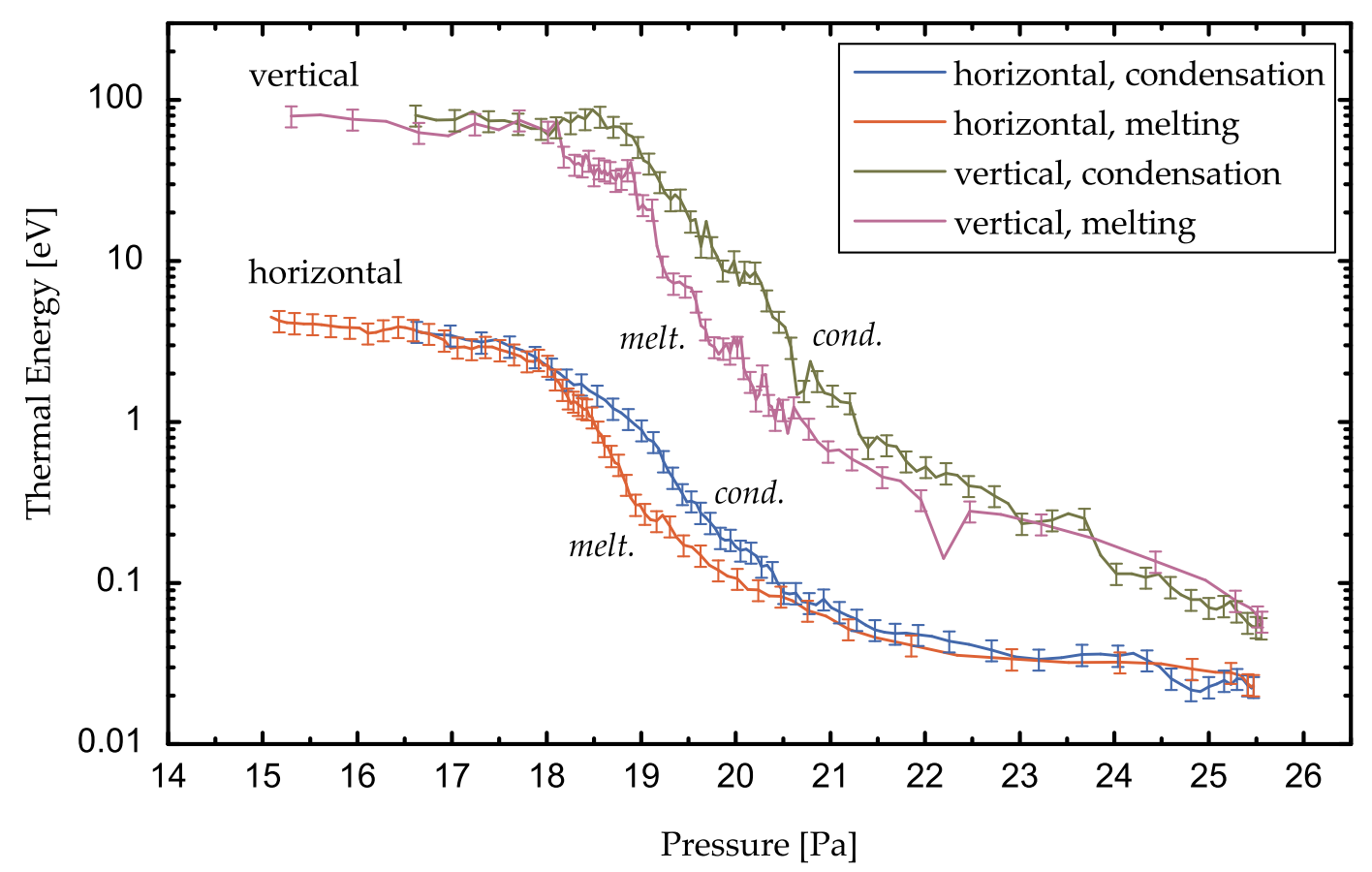

Figure 5.25: The condensation and melting transition induced by a continuous variation of the discharge pressure. The upper curves show the vertical and the lower curves show the horizontal component of motion. The critical pressure of condensation is higher than the critical pressure of melting for both components of movement. The error is assumed to be $\pm 15 \%$. This hysteresis loop is predicted by simulations by Joyce et al. [7, 8, 9] (see section 3.3.2]for details).

the results of section 5.3 .3 .

A similar hysteresis loop is obtained for a pressure variation in reversed direction, i.e. starting in the ordered state at high pressures. This shows, the observed effect is not caused by a delay in the data acquisition of the discharge pressure.

The width of the hysteresis loop can be manipulated by external plasma parameters. Although no systematic studies were performed on this topic, an increase of the discharge power seems to reduce the width of the loop. As mentioned, the height of the dust cloud plays a central role for the width of the hysteresis loop. A higher discharge power leads to a flattening of the dust cloud and this causes the hysteresis loop to vanish.

This section shows the first experimental observation of a hysteresis loop in the phase transition of a complex plasma. The hysteresis loop is predicted by simulations by Joyce et al. in [7, 8, 9] and the experiment discussed here confirms the assumptions and results of these simulations.

The simplified assumptions in the simulation of a infinite system without confinement forces lead to a much wider hysteresis than observed in the experiments. In particular the convective character of the ion-two stream instability is believed to be fundamental for the appearance of the hysteresis loop. The comparable small extension of the dust cloud in verti- 
cal direction in the experiment leads to a narrow but still observable hysteresis loop. To yield a deeper understanding of the dependency of the hysteresis loop on plasma parameters, the simulations has to be adapted to the experimental situation.

\subsection{The Diffusive Regime}

In this section, the ability of DLS to measure the diffusion constants in strongly coupled complex plasmas is discussed. To reach the diffusive regime, the length scale $q^{-1}$ has to be of the order of the dust-dust collision length. The measurements at low scattering angles of $4^{\circ}$ (section 5.1) revealed small exponential contributions to the autocorrelation function. Therefore, it is assumed that the diffusive particle motion can be examined at very low scattering angles.

The autocorrelation function obtained in a small angle DLS experiment is presented at the beginning of this section. The results from the DLS method are compared to simultaneous CCD observations. The small angle scattering is prone to large errors. Other contributions of uncertainty that are not relevant for scattering in the ballistic regime, can become important now. The errors of the small angle DLS will be discussed briefly. The last part of this section covers the impact of the inter-particle interactions on the particle movement. The length scale $q^{-1}$ is closer to the average particle distance and the inter-particle interactions should affect the quantities of particle dynamics measured.

A careful adjustment of the scattering setup makes a DLS experiment at $\theta=0.83^{\circ}$ feasible. The related characteristic length scale is $q^{-1}=7.0 \mu \mathrm{m}$. This measurement required some adaptations compared to the standard setup. The distance between dust particle system and first aperture had to be enlarged to $2.2 \mathrm{~m}$ and the laser had to be attenuated by a factor of 100 . The larger length scale $q^{-1}$ allowed to reduce the acquisition frequency down to $200 \mathrm{kHz}$. On the other hand, the sample time had to be increased to several minutes to obtain smooth autocorrelation functions.

An example of an autocorrelation function under these conditions is shown in figure 5.26, The data are plotted on a logarithmic scale to identify exponential decays more easily as a linear function. Obviously, an exponential decay can be seen. A closer look shows a second decay form at short times that is not exponential. Again, a combination of Gaussian and exponential fit is applied with good agreement. The residuals are below $0.3 \%$.

This result shows, that even for scattering angles at the lower limit, the pure diffusive regime cannot be reached, but there is still a contribution of ballistic scatterers. Nonetheless, the exponential decay is dominant and the diffusion constant can be determined.

As expected, the decay times are much smaller under these scattering angles. The exponential decay has a decay constant of $\tau_{q}=59 \mathrm{~ms}$ and the Gaussian part has a decay time of $\sigma=16 \mathrm{~ms}$. With the equations $\left\langle v_{x}^{2}\right\rangle=\left(2 \sigma^{2} q^{2}\right)^{-1}$ and $D=\left(2 q^{2} \tau_{q}\right)^{-1}$, as derived in section 2.3.2, the particle velocity and the diffusion constant are $v_{x}=307 \mu \mathrm{m} / \mathrm{s}$ and 


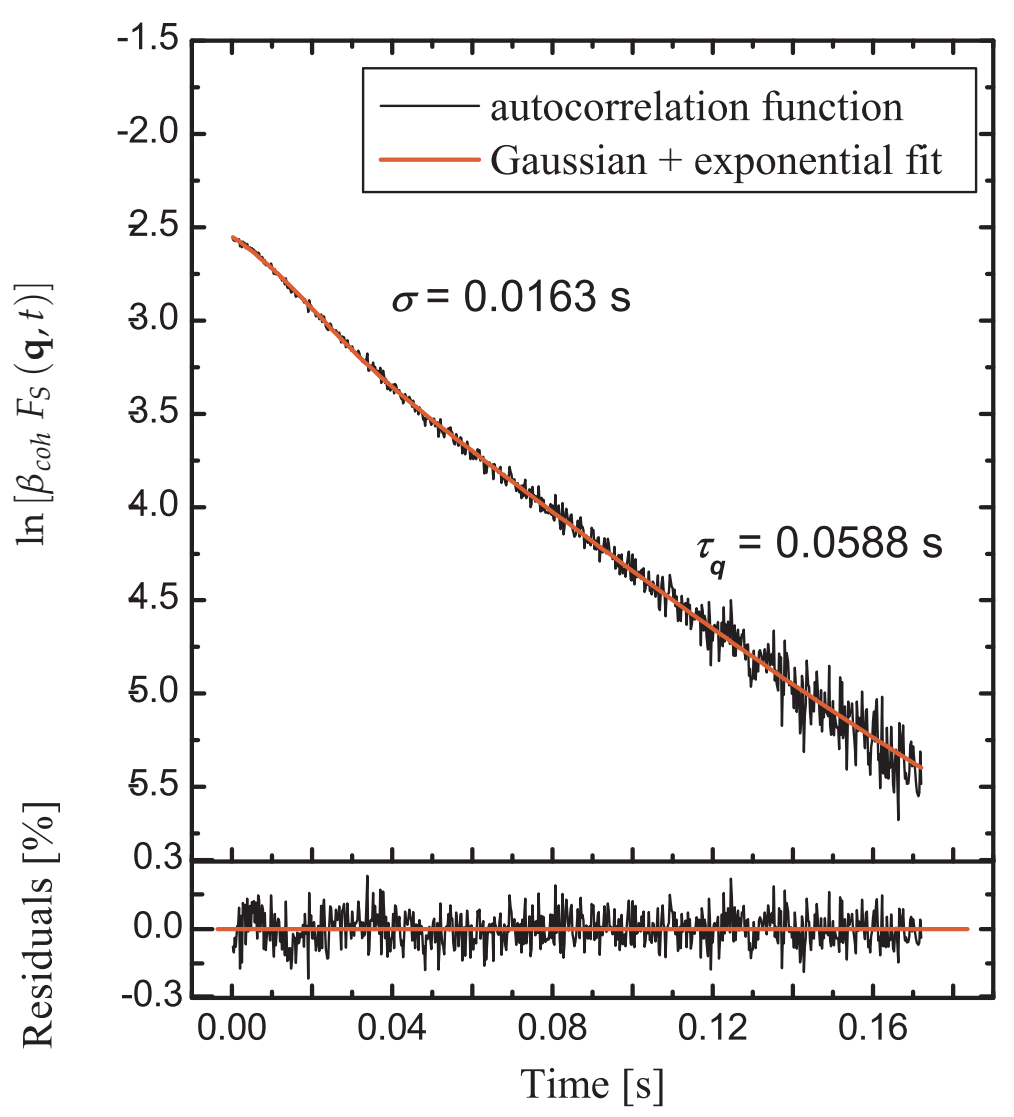

Figure 5.26: The logarithm of an autocorrelation function for a small angle scattering experiment at $\theta=0.83^{\circ}$. A mixed regime is observed. The ballistic regime appears as a Gaussian decay at short times and the diffusive regime becomes visible by an exponential contribution at longer times scales. The situation can be described by a combined fit of Gaussian and exponential decay with residuals typically lower than $0.3 \%$. This results in decay constants of $\sigma=0.0163 \mathrm{~s}$ for the ballistic motion and $\tau_{q}=$ $0.00588 \mathrm{~s}$ for diffusive motion.

$D=4.1 \cdot 10^{-6} \mathrm{~cm}^{2} / \mathrm{s}$.

To benchmark the small angle DLS, the diffusion constants are compared to the values obtained by means of the standard CCD technique. In an analogue procedure as applied in the ballistic regime (section 5.1), a horizontal plane of the dust ensemble was analyzed by the CCD diagnostic simultaneously. The DLS scattering volume had a cylindrical shape with a length of $l=15 \mathrm{~mm}$ and a diameter of $d_{l}=0.7 \mathrm{~mm}$. A number of about 700 particles were probed. An area of $5 \mathrm{~mm} \cdot 5 \mathrm{~mm}$ containing about 200 particles were detected in the CCD measurement. The ensemble in the CCD measurement was therefore smaller. This results in a bigger standard deviation that can be compensated by a longer data acquisition time. The average particle distance in the horizontal plane was of the order of $400 \mu \mathrm{m}$.

Figure 5.27 depicts the diffusion constants from the DLS technique (circles) and the CCD technique (squares) for discharge pressures decreased from $100 \mathrm{~Pa}$ to $15 \mathrm{~Pa}$. A good agreement is found, the data points show overlapping error bars. This proves the ability of the small angle DLS approach to measure diffusion constants in a strongly coupled complex plasma.

The relative error of the CCD method for a measurement of the diffusion constant has a systematic part due to the error of the scale factor $f_{s}$ for converting pixels into microns. Other contributions to the error are considered by the standard deviation of the mean-square 


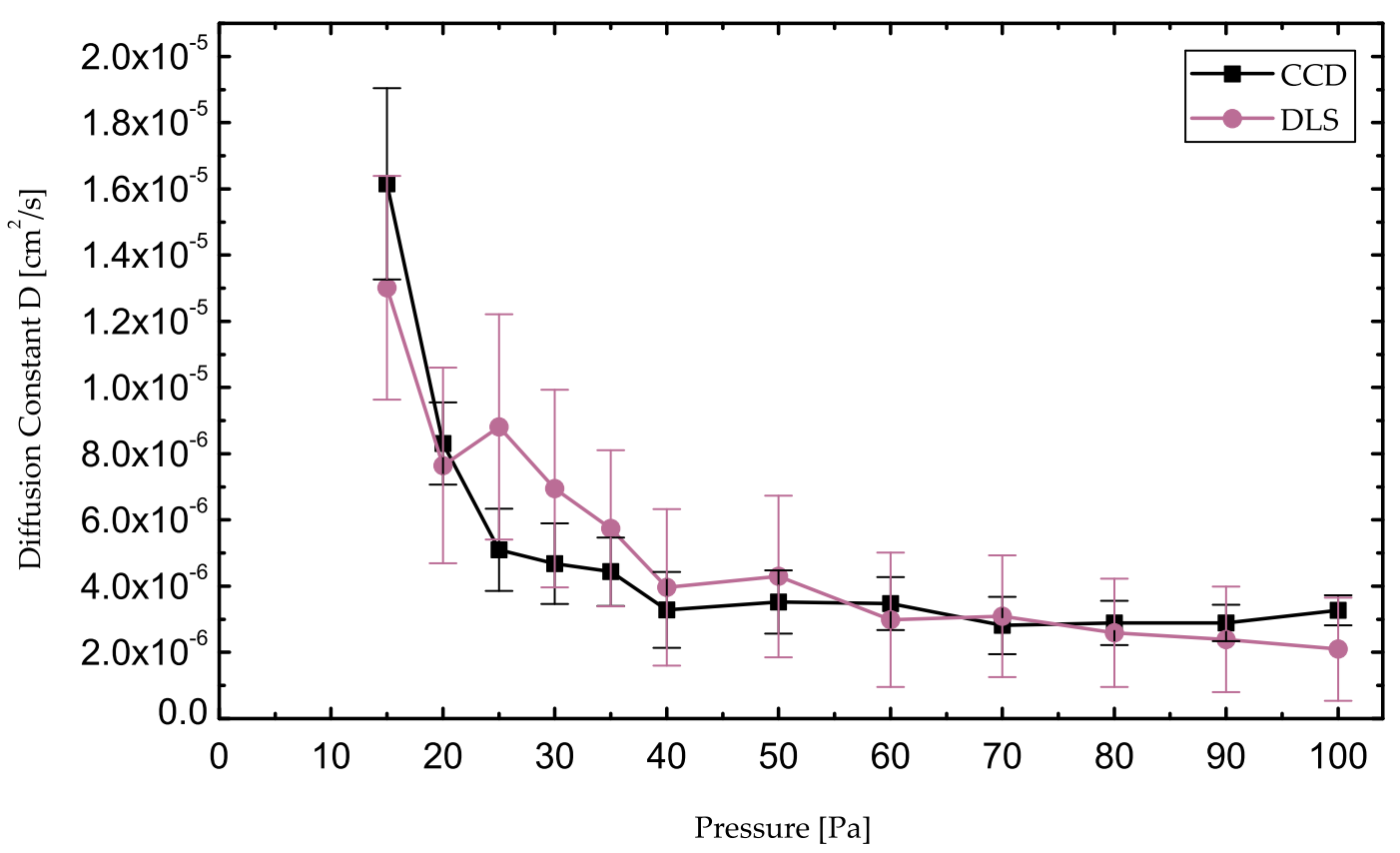

Figure 5.27: The diffusion constants measured with the DLS approach (circles) and the CCD method (squares). The pressure is varied from $100 \mathrm{~Pa}$ to $15 \mathrm{~Pa}$. A good agreement between both techniques justifies the use of the DLS diagnostic to measure diffusion constants in a strongly coupled complex plasma.

displacement $\left\langle\Delta p x^{2}\right\rangle$ in pixels that is calculated for the sequence of frames. This is an output of the particle tracking procedures of the video analysis. The diffusion constant is obtained from the mean-square displacement in one dimension via $D=\left\langle\Delta p x^{2}\right\rangle f_{s}^{2} /(2 T)$, where $T$ is the acquisition time. The error calculated by Gaussian error propagation includes the errors of $\left\langle\Delta p x^{2}\right\rangle$ and $f_{s}$ and is less than $\pm 35 \%$.

There are two main sources of error to be considered for the DLS measurement. The first contribution is due to the error in the determination of the scattering angle $\theta$. Again, the angle was determined by measuring distances in the triangle defined by the unscattered and scattered beams and the position of the first aperture. The accuracy of measuring distances is assumed to be $\pm 5 \mathrm{~mm}$. The error of the decay time $\tau_{q}$ is assumed to be of the order of $\pm 30 \%$. The application of Gaussian error propagation to the equation $D=\left(2 q^{2} \tau_{q}\right)^{-1}$ results in an error of the order of $\pm 25 \%$ at pressures around $80 \mathrm{~Pa}$ and $\pm 65 \%$ around $30 \mathrm{~Pa}$. At low pressures, the Gaussian decay form becomes dominant and the fit of the exponential decay is less confident. This restricts the measurement of diffusion constants by DLS to the ordered phase state of the dust system.

The error can be reduced by a more precise determination of the scattering angle with alternative methods. Another way would be to choose a higher wavelength to reach the diffusive regime at larger scattering angles already. But DLS in the infrared or far-infrared requires very different experimental conditions regarding detectors, windows, light sources and so forth. 


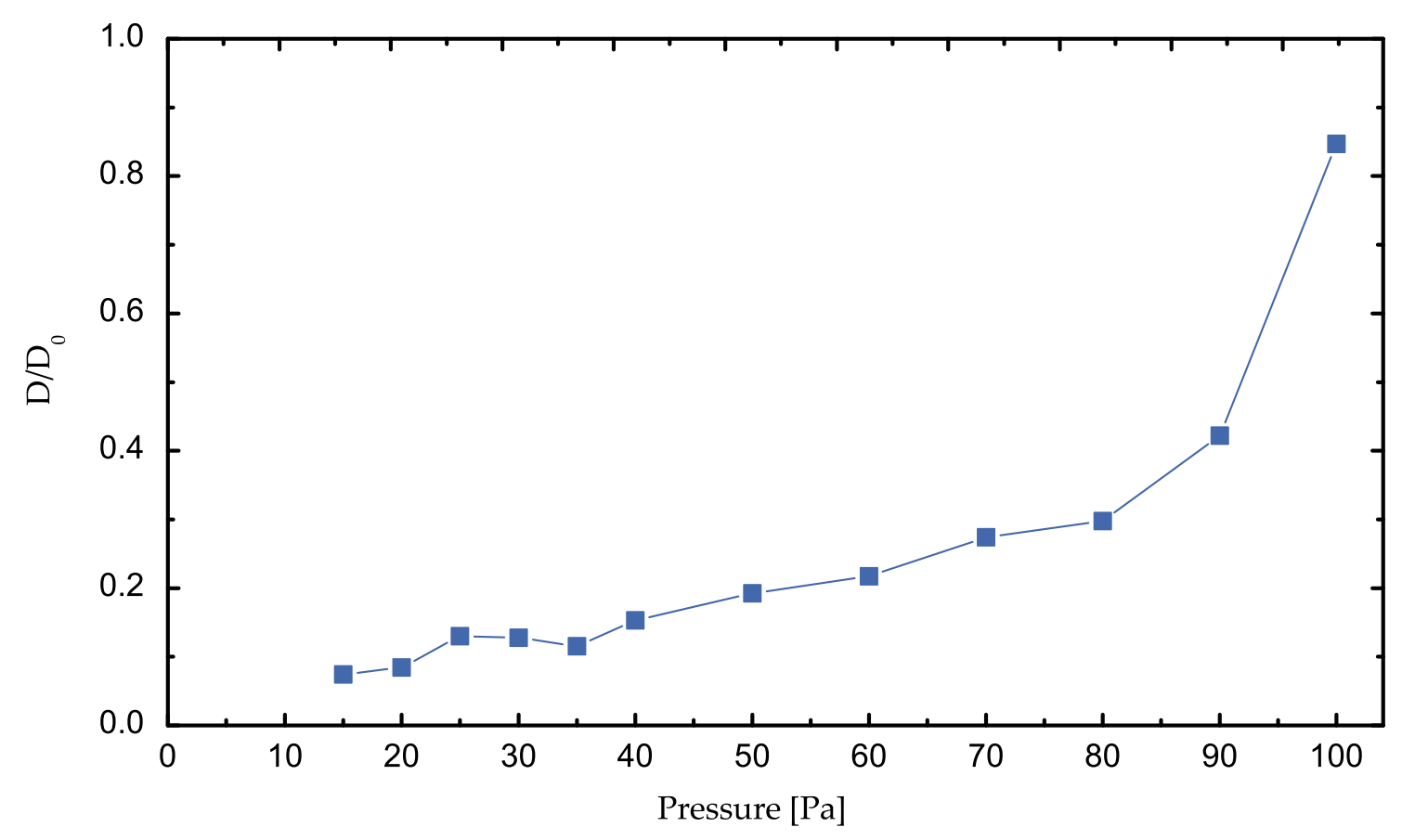

Figure 5.28: The ratio of the diffusion constant obtained with DLS and the free diffusion constant of neutral particles for pressures between $100 \mathrm{~Pa}$ and $15 \mathrm{~Pa}$. $D_{0}$ is inversely proportional to the discharge pressure. At low pressure, the diffusion constant is only about $10 \%$ of the free diffusion due to strong particle interactions. For large pressures, the diffusion constant reaches $85 \%$ of free diffusion.

In the final part of this section, the impact of particle interactions on the diffusive motion will be treated. The diffusion constants $D$ are set into relation to the free particle diffusion $D_{0}$ of neutral particles with the same temperature $T_{d}$.

To calculate the ratio $D / D_{0}$, the frictional resistance $\mu$ of the particle movement is introduced as

$$
\mu=\frac{D}{k_{B} T_{d}} .
$$

The electro-static interactions of charged particles lead to an increased resistance $\mu$ compared to neutral particles. The frictional resistance of neutral particles is given only by the gas friction and can be written as

$$
\mu_{0}=\frac{D_{0}}{k_{B} T_{d}}=\frac{1}{v_{d} m_{d}},
$$

where $v_{d}$ is the dust-neutral collision rate and $m_{d}$ is the dust particle mass. The collision rate $v_{d}$ is introduced in section 3.3.2 by equation (3.44), that is after Epstein [71]. This expression can be transformed to a function of gas pressure $p$, density of the dust particle material $\rho$, and radius of the dust particles $a$ as suggested in [91].

$$
v_{d} \cong 840 p[\text { Torr }] /\left(\rho\left[\mathrm{g} / \mathrm{cm}^{3}\right] r[\mu \mathrm{m}]\right)
$$

where the number 840 represents properties of argon gas. The ratio of the diffusion constants 
Figure 5.29: Dependence of the ratio $D / D_{0}$ on $\Gamma$ for different $\kappa$ (filled symbols refer to $\kappa=2.4$ and open symbols to $\kappa=4.8$ ) and $\xi$ (Note, $\Gamma^{*}$ instead of $\Gamma$ is used in the figure.). $\xi$ is changed from 0.055 for the $\mathrm{x}$-symbols to 4.5 for the squares. The quantity $\xi$ is defined as the ratio of inter-particle interaction and energy dissipation in the system. The dashed lines correspond to an analytical approximation proposed in [91]. (The figure is taken from Vaulina et al. [91].)

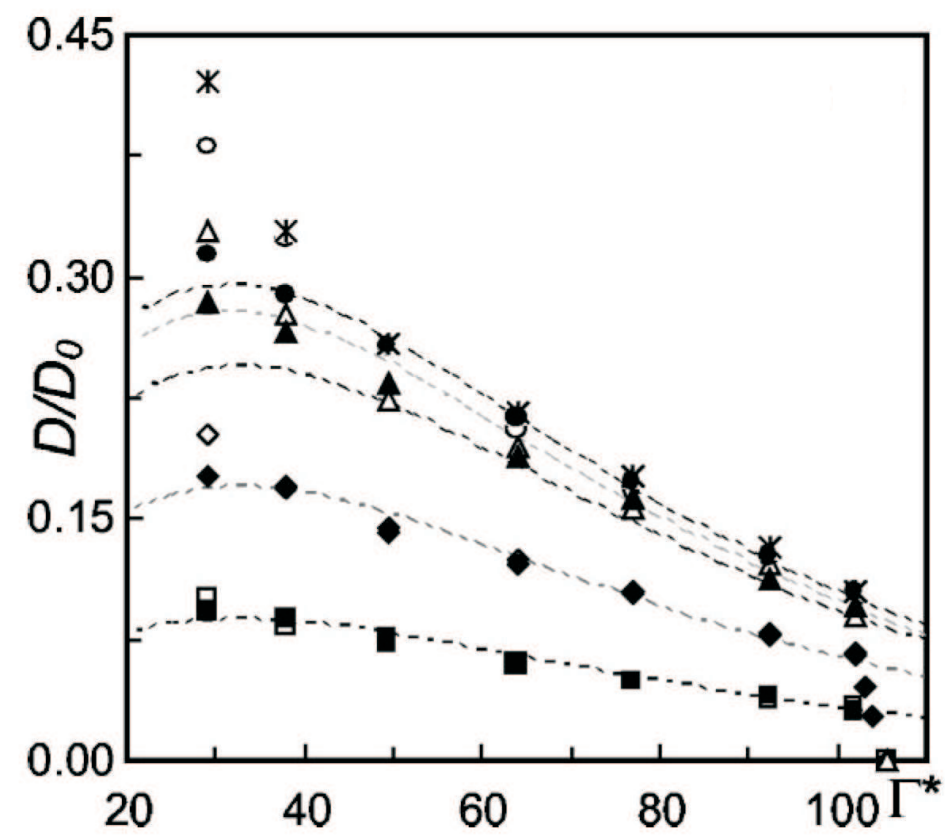

can now be calculated using the frictional resistance via $D / D_{0}=D /\left(k_{B} T_{d} \mu_{0}\right)$. The diffusion constant $D$ and the particle temperature $T_{d}$ are obtained from the combined fit of the autocorrelation function and $\mu_{0}$ is derived from the measured discharge pressures and known dust particle parameters using the equations (5.6) and (5.5).

Figure 5.28 shows the ratio $D / D_{0}$ versus the discharge pressure. All points regard to an ordered phase state of the particle system. The diffusion is strongly suppressed at low pressures and is only about $10 \%$ of free particle diffusion. Strong interactions between the particles increasing the friction resistance $\mu$. By increasing the discharge pressure, the ratio $D / D_{0}$ rises mostly linearly until a pressure of about $80 \mathrm{~Pa}$ is reached. A stronger growth of the ratio $D / D_{0}$ is seen for higher pressures. Here, the ratio $D / D_{0}$ tends to unity, where free particle motion is present.

In a publication of Vaulina et al. [91], the ratio $D / D_{0}$ is obtained by Brownian motion simulations in three dimensions. The change of $D / D_{0}$ for variations of the coupling parameter $\Gamma$ is analyzed for different screening strengths $48 \kappa$. The results of the simulations are shown in figure 5.29. The filled symbols refer to $\kappa=2.4$ and the open symbols refer to $\kappa=4.8$. The curves correspond to different rates of energy dissipation due to friction. This is introduced in the simulation by the ratio of inter-particle interaction and energy dissipation in the system $\xi$. The ratio $\xi$ increases from 0.055 (x-symbols) to 4.5 (squares) (see [91] for details).

The transition to a crystal appears at $\Gamma=106$. There, the ratios $D / D_{0}$ suddenly drop to zero, because the particles are fixed at their lattice positions. Note, the phase states from the

\footnotetext{
${ }^{48}$ The coupling parameter $\Gamma$ and the screening strength $\kappa$ are introduced in section 3.3 .1 Note, $\Gamma^{*}$ instead of $\Gamma$ is used in the figure.
} 


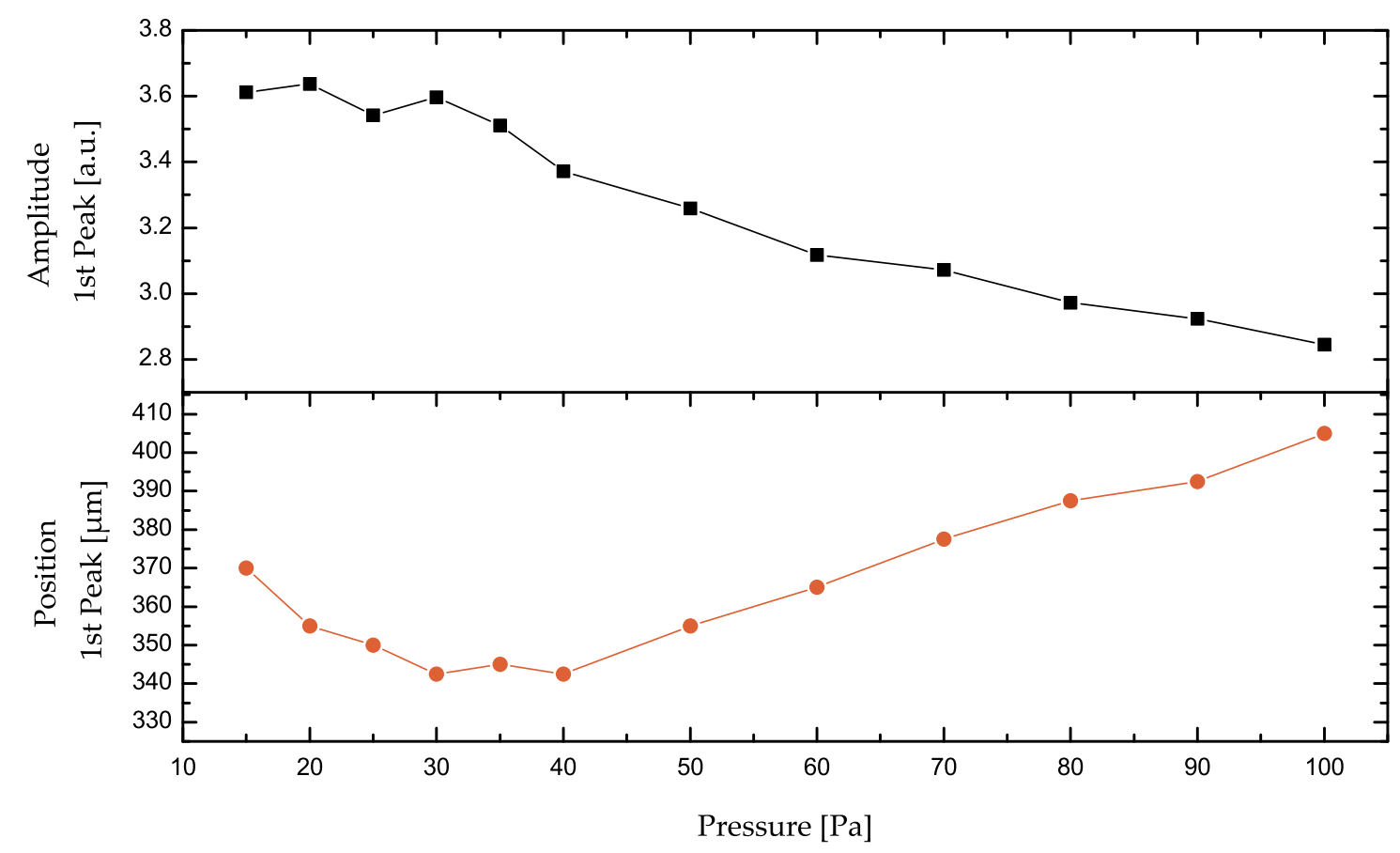

Figure 5.30: The amplitude (squares, upper graph) and position (circles, lower graph) of the first peak of the pair correlation function versus the discharge pressure. At low pressure the large amplitude of the pair correlation function indicates a high coupling constant. With increase of the pressure, the amplitude drops. At large pressure, the particles are less coupled and further away of each other.

melted state up to the crystal state are examined in the simulations. In the experiment (figure 5.28) only pressures above the condensation transition are studied.

Nevertheless, the coupling parameter in the experiment and simulation are believed to be comparable. A perfect crystal like in the simulations is not observed in the experiment. There were still particle movements and exchange of single particles or even whole particle chains. For this reason, a lower value $\Gamma$ is expected even for the most ordered structures observed. Thus, $\Gamma$ is assumed to be between 80 and 100 in the experiment, so below the transition to a perfectly ordered crystal. Here, the ratios $D / D_{0}$ from the simulations are between 0.2 and 0.03. This result is in qualitative agreement with the measurement shown in figure 5.28 for pressures between $15 \mathrm{~Pa}$ to $40 \mathrm{~Pa}$, albeit the experimental parameters are slightly different in the simulation.

The rise of the ratio $D / D_{0}$ for higher pressures in the experiment can be explained by a decrease of the coupling parameter $\Gamma$. This is confirmed by an analysis of the amplitude of the first peak of the pair correlation function, measured by the aid of the CCD method. The amplitude of the pair correlation function is proportional to the coupling parameter $\Gamma$.

Figure 5.30 shows in the upper graph the amplitude and in the lower graph the position of the first peak of the pair correlation function as a function of the discharge pressure. The position of the first peak gives the average particle distance. As can be seen, the coupling be- 
tween the particles drops with an increase of the discharge pressure. Just after condensation, the particles show the highest order. For higher pressures, the particles move very slowly due to strong gas friction, but they show less order with a larger mean inter-particle separation.

This forces the ratio $D / D_{0}$ to rise with pressure. The interaction between particles is reduced and the friction due to dust-dust collisions becomes less important. The frictional resistance tends to the resistance given by gas friction $\mu \rightarrow \mu_{0}$.

In previous DLS experiments on complex plasmas to determined the diffusion constants, the dust grains were in a disordered state [92] or weakly coupled as for nano-scaled dust grains [16]. Here, the applicability of DLS to measure diffusion constants is extended for the first time to well ordered strongly coupled systems of large dust grains in RF-discharges.

Very small scattering angles are necessary to reach a characteristic length $q^{-1}$ sufficiently large. Thus, the common problems of a small angle scattering experiment, like errors in the determination of the scattering angle, low angle reflections and backscattering are present, but can be controlled.

Under these experimental conditions, a mixed regime of ballistic and diffusive motion is observed. This allows to study the frictional resistance introduced as $\mu=D / k_{B} T_{d}$ because both, particle temperature $T_{d}$ and diffusion constant $D$, are measured simultaneously. The inter-particle interactions cause a reduction of the diffusion compared to the free particle diffusion of neutral particles. The measurements are in qualitative agreement with simulations.

It is shown that the frictional resistance due to dust-dust collisions is reduced at higher pressures and a state of nearly free particle diffusion is reached above $100 \mathrm{~Pa}$. 



\section{Chapter 6}

\section{Conclusion and Outlook}

The aim of this work can be divided into two parts: First, the realization and successful application of the DLS technique to examine complex plasmas has to be demonstrated. Secondly, the DLS technique has to be used to study phase transitions in $3 \mathrm{D}$ complex plasmas and to measure diffusion constants.

The realization of the experiment began with a careful choice of the components of the DLS setup. The forward scattering mode, as used in this work, is prone to errors and statistically fluctuations introduced by experimental instruments can be fatal. The setup was successfully validated using photon statistics.

Furthermore, the assumption of Gaussian distributed complex amplitudes in the scattered light field was proven, because this is a central assumption in DLS theory. Only if this assumption holds, the Siegert relation can be applied to obtain the self-intermediate scattering function $F_{S}(\mathbf{q}, t)$ from a homodyne experiment.

A single laser beam approach to determine the horizontal and the vertical component of particle motion at the same time was realized in this work successfully. This is a great simplification of the DLS setup to measure two components of motion.

The application of the DLS on a complex plasma in a CCP RF-discharge revealed free (ballistic) particle motion. The decay form of the autocorrelation function is found to be a pure Gaussian function for scattering angles between $4^{\circ}$ and $180^{\circ}$. This is demonstrated for the ordered as well as for the melted phase state. An additional exponential contribution was observed close to the phase transition for scattering angles below $4^{\circ}$. The measurement of the thermal energy of the dust particles with DLS was benchmarked successfully with the standard CCD technique.

The error in determining the particle velocity $v_{x}$ and thermal energy $T_{e V}$ respectively, was estimated statistically performing a series of measurements. The $1 \mathrm{D}$ velocity $v_{x}$ can be measured with an error of $3 \%$ for a 2 second data acquisition in the backscattering mode. At lower scattering angles, the error increases and reaches up to $10 \%$ at $4^{\circ}$. This is due to the larger error in determining the scatteing angle $\theta$.

The error of $T_{e V}$ is larger due to the uncertainties of the particle radius. An error between 
$10 \%$ and $20 \%$ has to be assumed.

This part of the work proves the application of DLS on strongly coupled complex plasmas. This introduces an alternative diagnostic to the field of complex plasmas that overcome the temporal and spatial limitations of the standard CCD technique. DLS is in particular suited to study the fast particle movement in the melted state and to get a deeper understanding of phase transitions in 3D dust systems.

The phase transitions are examined by DLS in the second part of the work by detailed parameter studies induced by variation of the discharge pressure $p$. The thermal energy was determined during the phase transition giving a transition curve $T_{e V}(p)$. An empirical model was developed to fit the transition curve $T_{e V}(p)$ and to describe it quantitatively by the fit parameters. This is essential to compare a huge set of measurements.

The energy was found to increase exponentially in the melted state. In contrast to the $2 \mathrm{D}$ case, a smooth transition between ordered and disordered state was observed. The prediction of a two-step transition in the thermal energy by simulations [6] on 2D systems could not be confirmed. Furthermore, the "floe and flow" and the "vibrational" state are observed in experiments on 2D systems are not present in the examined 3D systems. The two-step transition and the additional phase states seem to be unique for 2D systems.

As a first parameter variation, the external bias voltage, applied to the upper (driven) electrode, was examined. In the range of $-20 \mathrm{~V}$ to $20 \mathrm{~V}$, the transition curves are shifted to higher pressures for higher voltages. The shape of the transition curves does not change significantly. In the ranges of $-40 \mathrm{~V}$ to $-20 \mathrm{~V}$ and $20 \mathrm{~V}$ to $30 \mathrm{~V}$, a stable regime was observed.

The confinement voltages have nearly no affect on the phase transitions, which might be explained by the local impact of the confinement potential on the plasma. These measurements have defined the range of stable parameters for further experiments.

The variation of the particle number density $n_{d}$ and height of the dust cloud $h_{c}$ can have strong impact on the phase transitions. The phase transitions were examined under variation of the product of both parameters $n_{d} \cdot h_{c}$. It was shown that the transition curve is shifted to lower pressures if $n_{d} \cdot h_{c}$ decreases. These observation are in agreement with the predictions made in the theory by Joyce and Ganguli et al. [7, 8, 9]. For values of the product above $\approx 800$, a saturation was observed, i.e. the impact of $n_{d} \cdot h_{c}$ on the phase transitions vanishes.

The variation of the discharge power is connected to a variation of the product $n_{d} \cdot h_{c}$. Thus, a similar behavior was observed. The product $n_{d} \cdot h_{c}$ is found to increase with the discharge power.

The variation of $n_{d} \cdot h_{c}$ with discharge power is stronger for larger particles. This is confirmed by CCD observations. The critical pressures $p_{c}$ for a phase transition are in general lower for bigger particles. The increase of charge and mass leads to a shift of the transition curves as predicted by theory. These measurements give another experimental confirmation of the theory by Joyce and Ganguli et al. [7, 8, 9]. 
A fundamental phenomenon in ground based 3D complex plasmas is the gradual phase transition. The vertical movement of energy fronts was studied in detail for the first time. The capability of the experiment to measure and analyze a huge set of autocorrelation functions was demonstrated successfully.

The measurements show, the movement speed of the energy fronts is nearly constant for low thermal energies. The horizontal component of particle motion shows a faster movement of the energy fronts compared to the vertical component. This observation was confirmed qualitatively by another measurement on larger dust grains.

In the melted state, the movement of energy fronts shows to be different. The horizontal component shows a strong increase of the transport speed of energy for higher energies. This means, higher energies are transported faster than lower energies.

In contrast, the vertical component shows a constant transport speed for all energies. Again, this observation is confirmed by a second, independent measurement on larger dust grains. This surprising result might be explained by different instability modes in horizontal and vertical direction. The heat transport between the two components plays another crucial role. For a deeper understanding, further simulations adapted to the experimental conditions are necessary.

The detailed parameter studies of the phase transition resulted in another remarkable observation: The ratio of the thermal energies in horizontal and vertical direction $T_{H} / T_{V}$ shows to be the same in the melted state for all parameters studied in this work. The ratio is 0.05 just below the phase transition and increases if the pressure is further decreases. This surprising discovery makes $T_{H} / T_{V}$ to a universal parameter. This might be explained by a constant ratio of the amplitudes of the ion-tow stream instability modes in horizontal and vertical direction. It is an interesting future task to confirm this result by the aid of simulations and to give a theoretical explanation of this phenomenon.

The melting by increase of the discharge power was realized and the transition curve was measured. The transition curve is found to be different in general. The behavior of the transition curve cannot be described by the same model.

The theoretical work and simulations by Joyce and Ganguli et al. [7, 8, 9] predict a hysteresis loop in the phase transition. This work presents the first experimental observation of this hysteresis loop. The width of the hysteresis is smaller than the theoretical prediction. This might be explained by the limited extension in vertical direction of the dust cloud in the experiment. This observation gives another confirmation of the theory.

These detailed studies on phase transitions revealed new phenomena of 3D phase transitions such as the complicated energy distribution and transport of the two components in the melted state or the constants of the ratio $T_{H} / T_{V}$. These observations can be the experimental basis for comprehensive theories and simulations of 3D phase transitions. In particular, the confirmation of the simulations by Joyce et al. motivates a further development of this theory and an adaption to the experimental conditions. A detailed treatment of the different insta- 
bility modes in horizontal and vertical direction might shed some light on the experimental observations.

In this work, a DLS experiment to measure diffusion constants in a complex plasma was realized with very small scattering angles below $1^{\circ}$. A mixed regime of free and diffusive particle motion was observed. The Gaussian and exponential decays could be clearly separated. The measured diffusion constants were benchmarked by standard CCD observations. The observation of both regimes at the same time allows to calculate the particle radius, using the Einstein relation (equation 2.5049 .

The successful measurement of diffusion constants by DLS is a remarkable results, holding in mind the comparable large inter-particle distances and the strong coupling between the particles. This normally has strong impact on the decay form of the autocorrelation function.

Nevertheless, this approach is limited to the ordered state and the data acquisition times of the order of $10 \mathrm{~min}$ is rather long. To overcome these limitations, a future experiment could analyze number fluctuations to obtain the diffusion constants. Here, the characteristic length scale is given by the laser diameter $d_{l}$. If $d_{l}$ is sufficiently small but still larger than $l_{m f}$, the number fluctuations can be measured and deliver the diffusion constants. At the same time, the interference fluctuations can be used to measure the thermal energy $T_{e V}$. This approach simplifies the setup, because the very small scattering angles are not necessary anymore.

Another improvement proposed here concerns the calculation of the autocorrelation functions. This can be improved in general by introducing parallel processing routines using the graphical processing unit (GPU). The calculation of autocorrelation functions is well suited to be performed parallel. The implementation of parallel routines allows to compute autocorrelation functions with much higher resolution at same or lower run time.

In some experiments, a small number of slightly separated crystal layers at the bottom of the dust structure was observed. It is believed that these crystal parts consist of agglomerations of two or three particles. These agglomerations are not spherical anymore and show rotational movement due to the impact of the ion flow in the plasma-wall sheath. The analysis of the rotation frequency for different discharge pressures can be used to study the ion flow speed and therefore can be used to characterize the electric field in the (dusty) sheath. This can be performed with a DLS experiment in two ways. Firstly, the rotation frequency can be obtained by illumination with a thin laser beam. As for number fluctuations, the rotation at the boundary of the laser beam leads to a fluctuation of the scattered intensity. Secondly, depolarized dynamic light scattering can be used to study the rotational movement on much smaller length scales. As mentioned earlier, the anisotropic properties of the scatterers lead to a contribution of scattered light with polarization perpendicular to the polarization of the

${ }^{49} D=\frac{k_{B} T_{d}}{6 \pi \eta a}$ 
incident laser light. This technique is established in other research fields to measure rotational diffusion constants [13, 14].

Another future aim would be a deeper study of the particle dynamics in dust acoustic waves (DAW) by the aid of DLS. The applicability of the experimental setup, as presented here, for a phase resolved study of DAW has been demonstrated in some preliminary experiments. The examination of both components of motion gives insight into the coupling between the components in different phases of the DAW. The thermal energy of the particles is not only modulated by the harmonic excitation of the wave, but also the amplitude of the ion-two stream instability is expected to be modulated with the particle density. This can be studied by systematic investigation of the DAWs at different discharge conditions and for particles of different (known) size. 



\section{Appendix}

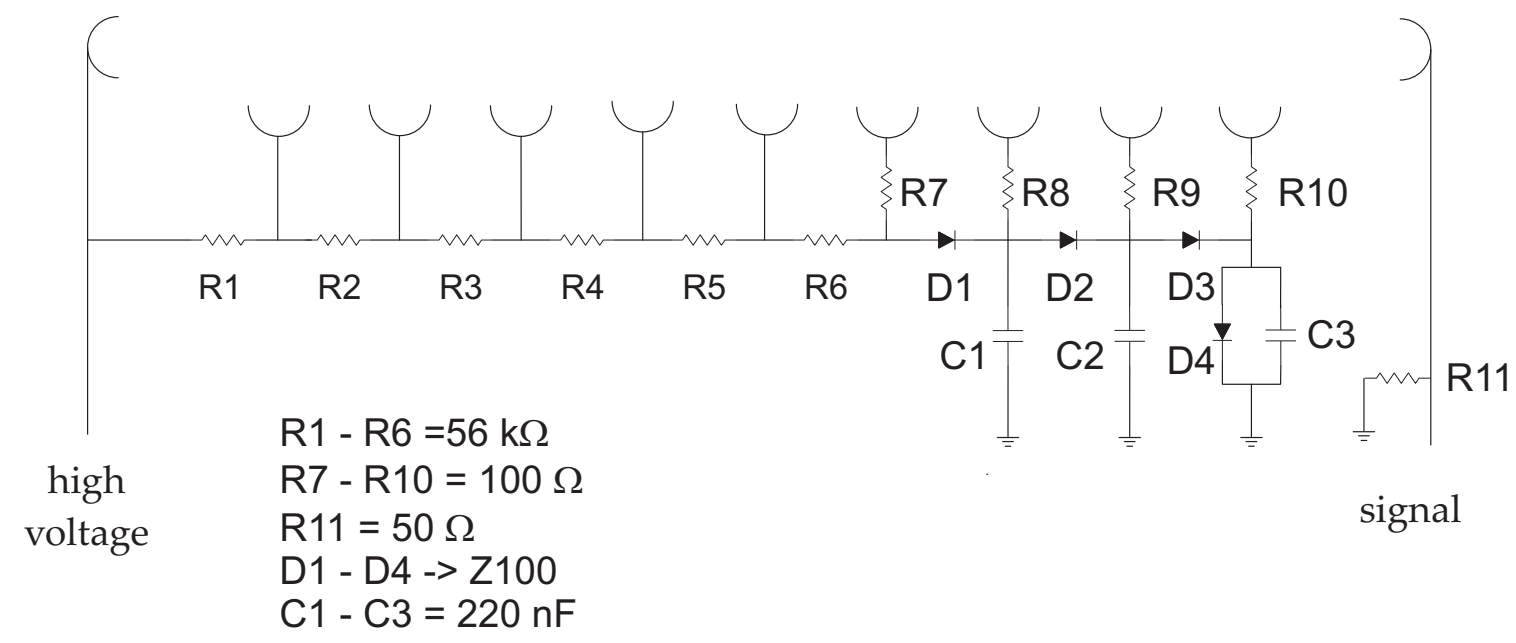

Figure A.1: Voltage divider network of the photomultiplier tubes. The design of the network allows to work in the photon counting mode (fast measurements or low intensity) as well as to measure large intensities (currents). 
(a)

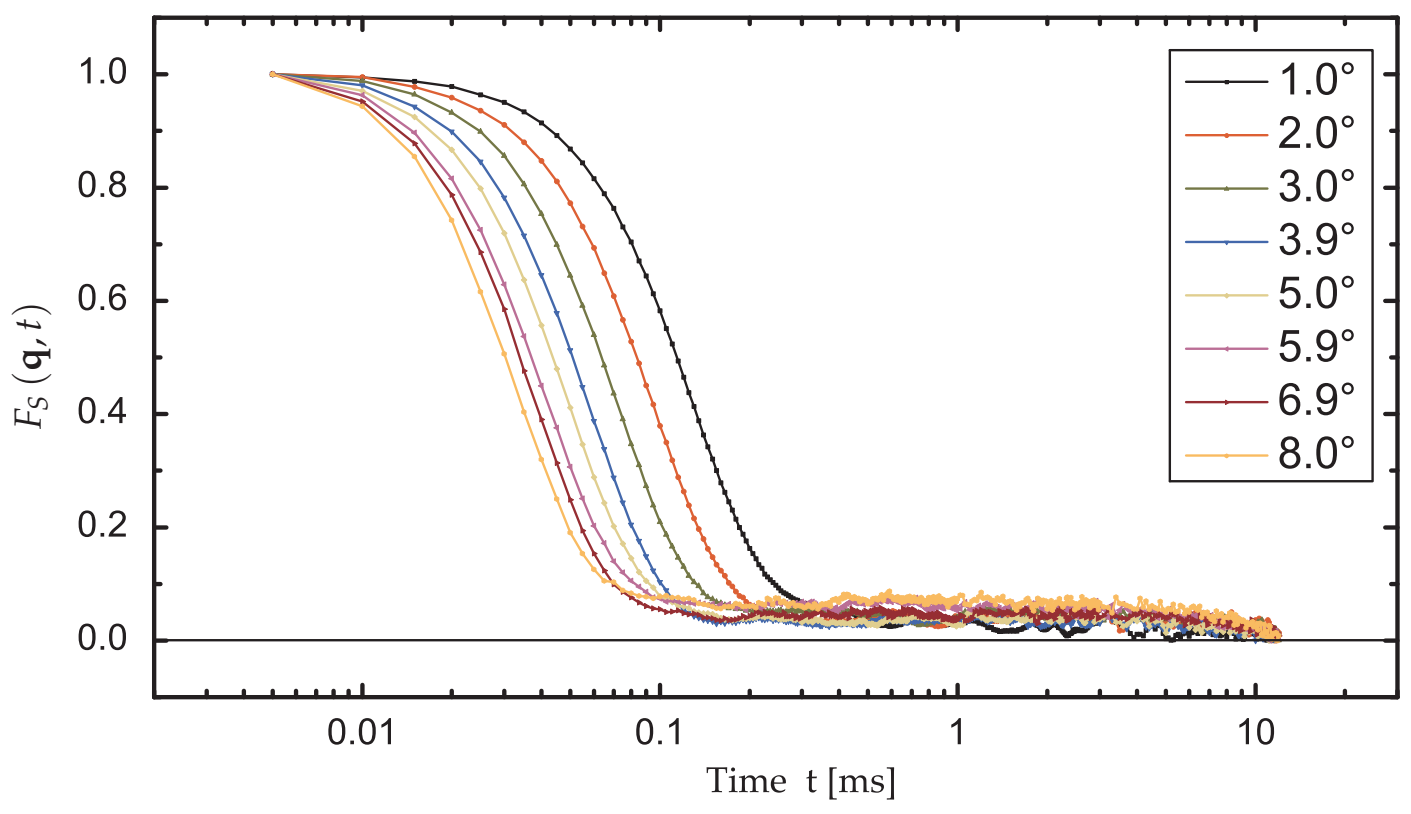

(b)

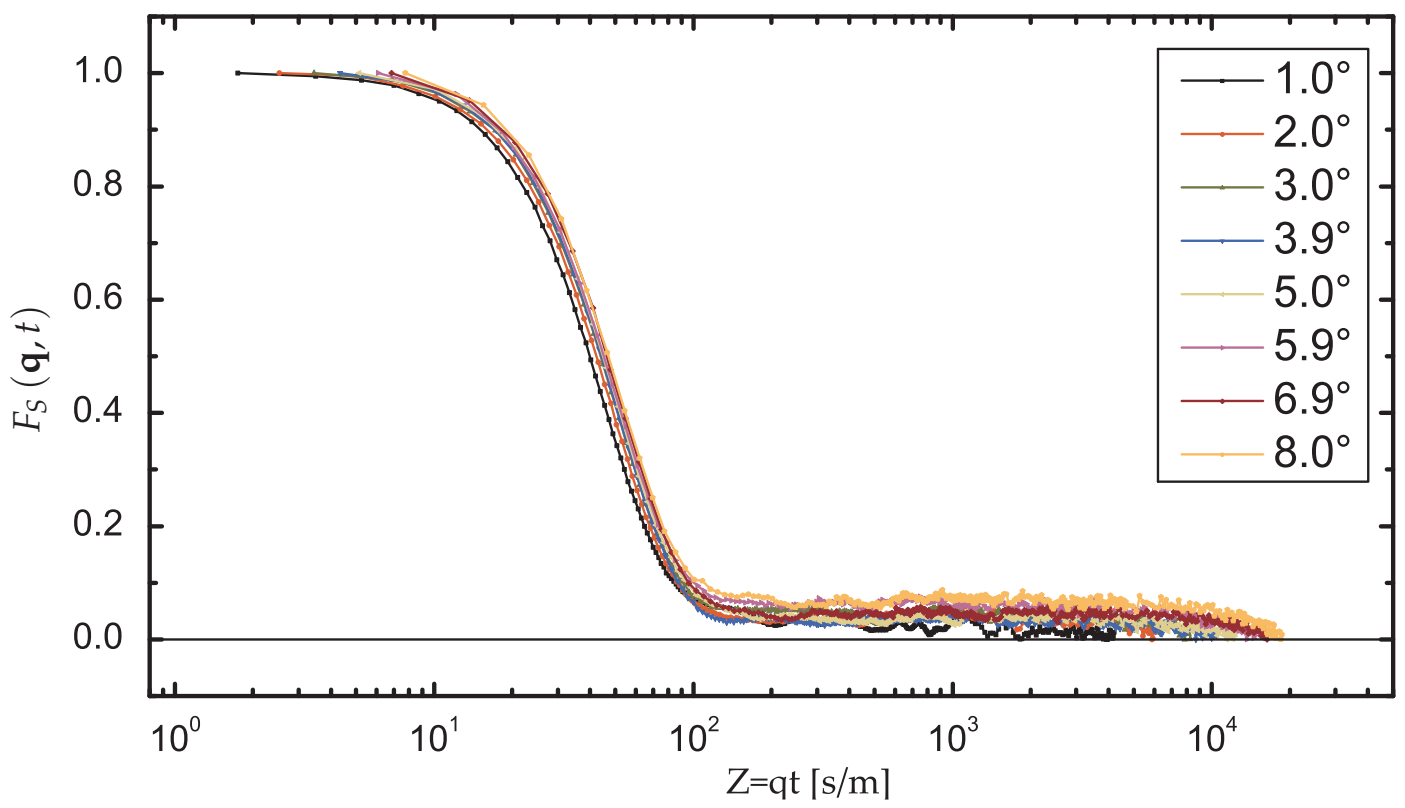

Figure A.2: Proof of ballistic particle motion for scattering angles between $1^{\circ}$ and $8^{\circ}$ in the disordered phase state at a discharge pressure of $2 \mathrm{~Pa}$. (a) The self-intermediate scattering functions $F_{S}(\mathbf{q}, t)$ at different scattering angles for particle movement in the horizontal plane. The decay time rises from $2 \cdot 10^{-5} \mathrm{~s}$ to $1 \cdot 10^{-4} \mathrm{~s}$ while the scattering angle is decreased. (b) The scattering functions $F_{S}(\mathbf{q}, t)$ superimpose if they are plotted over $Z=q t$, which proves ballistic particle motion. Small deviations appear due to the measurement error of the scattering angle $\theta$. 
(a)

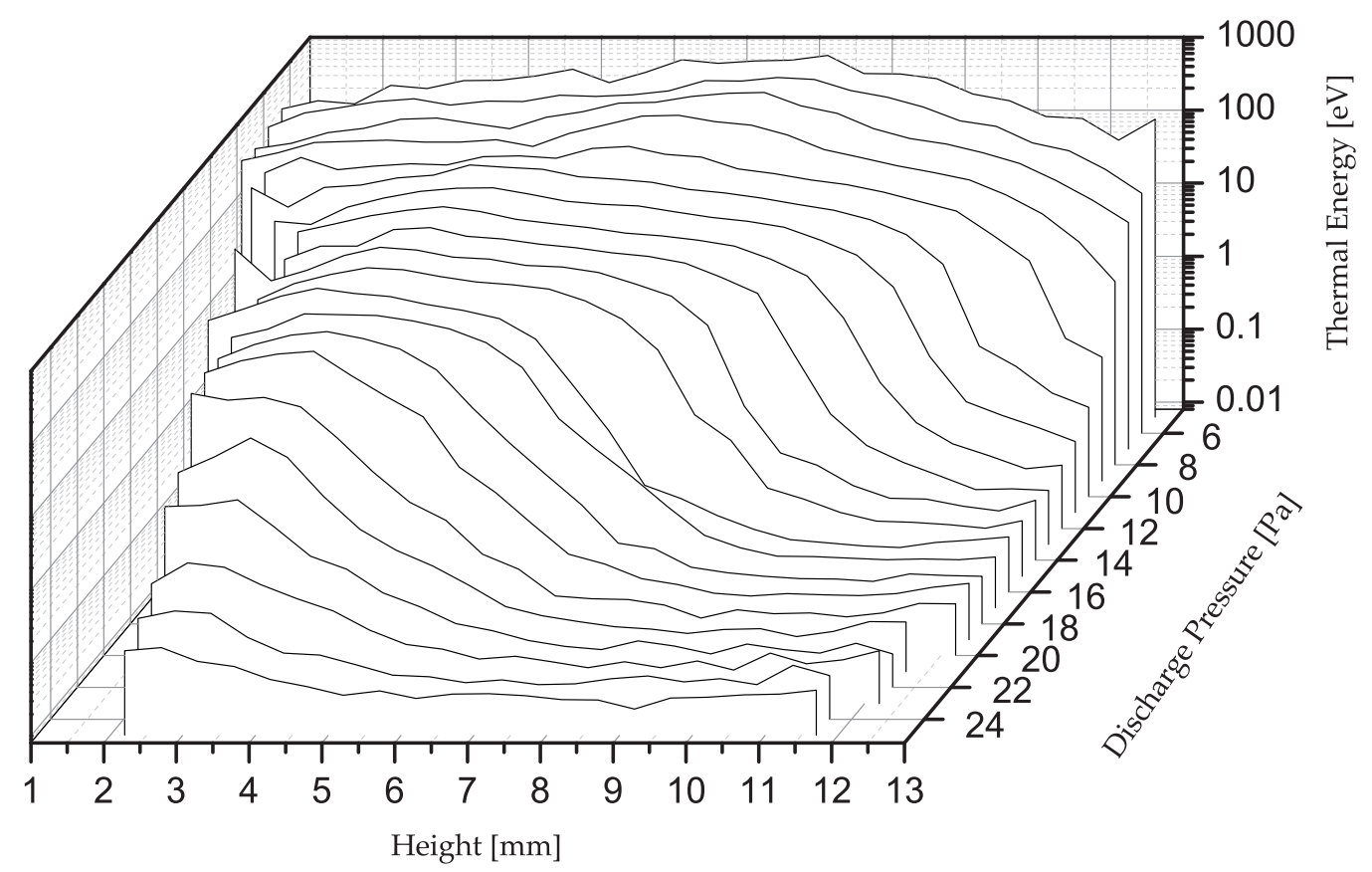

(b)

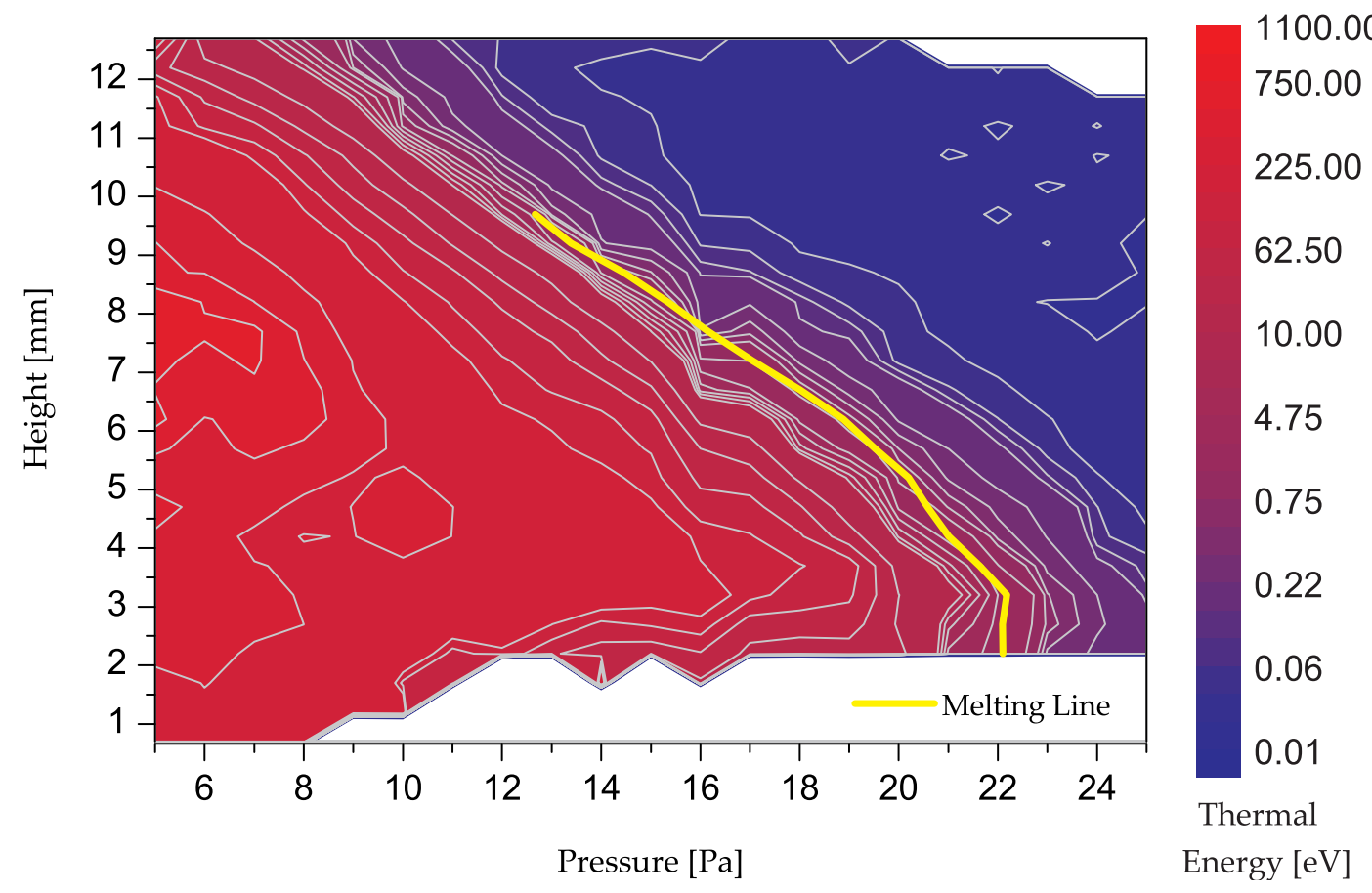

Figure A.3: (a) Thermal energy in the vertical plane for different positions of the scattering volume above the lower electrode. The pressure was varied from $5 \mathrm{~Pa}$ to $25 \mathrm{~Pa}$. (b) Contour plot of the same data. The lower left corner of the graph corresponds to the rearward left corner of the upper graph. The energy is color coded. The thick line (yellow) marks the melting transition line. 



\title{
List of Symbols and Abbreviations
}

\author{
$\beta \quad$ Scattering parameter. [page 31] \\ $\beta_{\text {coh }} \quad$ Coherence factor. [page 64] \\ $\Delta \quad$ Inter-particle distance. [page 47] \\ $\epsilon_{0} \quad$ Dielectric constant. [page 27] \\ $\eta \quad$ Viscosity of the background medium. [page 24] \\ $\Gamma \quad$ Coupling parameter. [page 47] \\ $\kappa \quad$ Screening parameter. [page 47] \\ $\lambda_{0} \quad$ Wavelength of incident laser light in vacuo. [page 10] \\ $\lambda_{D} \quad$ Total Debye length. [page 27]

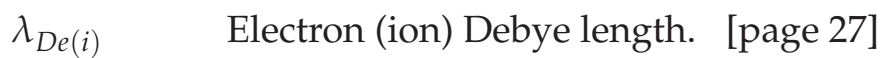 \\ $v_{d} \quad$ Dust-neutral collision frequency. [page 51] \\ $v_{i} \quad$ Ion-neutral collision frequency. [page 51] \\ $\omega_{d} \quad$ Dust plasma frequency. [page 50] \\ $\omega_{f} \quad$ Frequency of the scattered light. [page 15] \\ $\omega_{i} \quad$ Frequency of incident light. [page 12] \\ $\omega_{q} \quad$ Full width of the Gaussian function $\left(\omega_{q}=2 \sigma\right)$. [page 9]

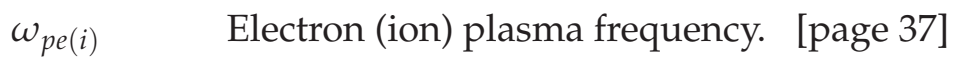 \\ $\phi(r) \quad$ Dust particle potential. [page 27] \\ $\phi_{s} \quad$ Dust particle surface potential. [page 27] \\ $\rho \quad$ Impact parameter for a collision on the dust grain surface. [page 31] \\ $\rho_{c} \quad$ Maximal impact parameter for a collision on the grain surface. [page 29]
}


$\sigma \quad$ Half width of the Gaussian function $\omega_{q}=2 \sigma$. [page 25]

$\tau \quad$ Lag time of the continuous autocorrelation function. [page 7]

$\tau_{q} \quad$ Decay time of the autocorrelation function. [page 7]

$\theta \quad$ Scattering angle of a DLS experiment. [page 10]

q Scattering vector of a DLS experiment. [page 10]

$\widetilde{I}_{\text {Filter }}\left(\omega_{f}\right)$ Power spectrum of a filter experiment. [page 16]

$\widetilde{I}_{\text {het }}\left(\omega_{f}\right) \quad$ Power spectrum of a heterodyne experiment. [page 16]

$\widetilde{I}_{\text {hom }}\left(\omega_{f}\right) \quad$ Power spectrum of a homodyne experiment. [page 16]

$\zeta \quad$ Friction constant $\zeta=6 \pi \eta a$. [page 24]

a Dust particle radius. [page 24]

$A_{D} \quad$ Detector area. [page 18]

$A_{\text {coh }} \quad$ Coherence area. [page 19]

$C_{d} \quad$ Dust particle capacitance. [page 30]

$C C D \quad$ Charge coupled device [page 2]

D Diffusion constant. [page 23]

$d_{l} \quad$ Diameter of the laser beam. [page 19]

DLS Dynamic light scattering. [page 2]

$E_{S}(\mathbf{q}, t) \quad$ Total amplitude of the scattered electric field. [page 12]

$F_{S}(\mathbf{q}, t) \quad$ Self-intermediate scattering function. [page 22]

$F_{\text {het }}(\mathbf{q}, t) \quad$ Heterodyne scattering function. [page 21]

$F_{\text {hom }}(\mathbf{q}, t) \quad$ Homodyne scattering function. [page 21]

$G(\mathbf{r}, t) \quad$ Van Hove space time correlation function. [page 22]

$G_{S}(\mathbf{r}, t) \quad$ Self Van Hove function. [page 22]

$g_{\text {hom }}(\mathbf{q}, t) \quad$ Autocorrelation function measured in a real experiment. [page 69]

$h_{c} \quad$ Height of the dust particle system. [page 93]

$I_{\text {het }}(t) \quad$ Heterodyne correlation function. [page 13] 
$I_{\text {hom }}(t) \quad$ Homodyne correlation function. [page 13]

$J \quad$ Number of points in a data sample (e.g. to calculate the discrete autocorrelation function). [page 7]

K Maximal lag time to calculate the autocorrelation function. [page 68]

$k_{B} \quad$ Boltzmann constant. [page 24]

$l_{i} \quad$ Ion free mean path. [page 32]

$l_{m f p} \quad$ Mean free path of collisions between scatterers. [page 18]

M Mach number. [page 36]

$m \quad$ Lag time of the discrete autocorrelation function. [page 7]

$m_{i} \quad$ Ion mass. [page 29]

$m_{n} \quad$ Mass of the neutrals. [page 45]

$m_{p} \quad$ Mass of the dust grains. [page 24]

$N \quad$ Number of dust particles in the ensemble. [page 12]

$n \quad$ Refractive index. [page 17]

$n_{0} \quad$ Plasma density (bulk). [page 27]

$n_{d} \quad$ Dust particle number density. [page 33]

$n_{n} \quad$ Number density of the neutrals. [page 45]

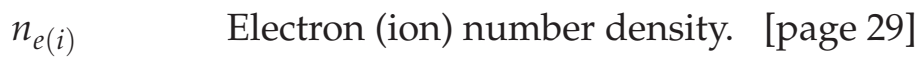

OML Orbital motion limited. [page 28]

$P_{H} \quad$ Havnes parameter. [page 34]

$p_{\text {cond }} \quad$ Critical pressure for the condensation transition. [page 54]

$p_{\text {melt }} \quad$ Critical pressure for the melting transition. [page 54]

$q^{-1} \quad$ Inverse absolute value of the scattering vector, that defines the characteristic length scale of a DLS experiment. [page 12]

$Q_{d} \quad$ Dust particle charge. [page 30]

RF $\quad$ Radio frequency [page 1] 
$s(t) \quad$ Fluctuating signal detected by the photomultiplier tube. [page 13]

$T_{d} \quad$ Temperature of the dust component. [page 24]

$T_{n} \quad$ Temperature of the neutrals. [page 46]

$T_{e(i)} \quad$ Electron (ion) temperature. [page 27]

$T_{e V} \quad$ Thermal energy of the dust particles. [page 72]

$T_{H} / T_{V} \quad$ Ratio of the thermal energy in horizontal and vertical direction. [page 106]

$u_{i} \quad$ Ion streaming velocity. [page 34]

$U_{e f f}(r) \quad$ Effective potential of the dust grains. [page 31]

$v_{B} \quad$ Bohm velocity. [page 34]

$v_{x} \quad$ One-dimensional velocity of the dust particles. [page 25]

$v_{e(i)} \quad$ Electron (ion) velocity. [page 30]

$v_{T_{e(i)}} \quad$ Electron (ion) thermal velocity. [page 30]

$z \quad$ Dimensionless charge. [page 32]

$Z_{d} \quad$ Charge number of the dust grains. [page 30]

$Z_{i} \quad$ Charge number of the ions. [page 38]

DAW Dust acoustic wave. [page 49]

DC Direct current. [page 1]

GPU Graphical processing unit. [page 126]

GUI Graphical user interface. [page 60]

HDF Hierarchical data format. [page 60]

IDL Interactive data language. [page 60] 


\section{Bibliography}

[1] J. H. Chu and L. I. Direct observation of Coulomb crystals and liquids in strongly coupled RF dusty plasmas. Phys. Rev. Lett., 72(25):4009-, June 1994.

[2] Y. Hayashi and K. Tachibana. Observation of Coulomb-crystal formation from carbon particles grown in a methane plasma. Jpn. J. of Appl. Phys., 33(Part 2, No. 6A):L804-L806, 1994.

[3] H. Thomas, G. E. Morfill, V. Demmel, J. Goree, B. Feuerbacher, and D. Möhlmann. Plasma crystal: Coulomb crystallization in a dusty plasma. Phys. Rev. Lett., 73(5):652655, August 1994.

[4] V. E. Fortov. Liquid- and crystallike structures in strongly coupled dusty plasmas. Phys. Plasmas, 6(5):1759-, 1999.

[5] A. Melzer, V. A. Schweigert, I. V. Schweigert, A. Homann, S. Peters, and A. Piel. Structure and stability of the plasma crystal. Phys. Rev. E, 54(1):R46-, July 1996.

[6] V. A. Schweigert, I. V. Schweigert, A. Melzer, A. Homann, and A. Piel. Plasma crystal melting: A nonequilibrium phase transition. Phys. Rev. Lett., 80(24):5345-, June 1998.

[7] G. Joyce, M. Lampe, and G. Ganguli. Instability-triggered phase transition to a dustyplasma condensate. Phys. Rev. Lett., 88(9):095006-, February 2002.

[8] G. Ganguli, G. Joyce, and M. Lampe. Phase transition in dusty plasmas. In Nonequilibrium Phenomena in Plasmas, volume 321 of Astrophysics and Space Science Library, pages 273-290. Springer Netherlands, 2005.

[9] M. Lampe, G. Joyce, and G. Ganguli. Structure and dynamics of dust in streaming plasma: Dust molecules, strings, and crystals. IEEE Trans. Plasma Sci., 33(1):57-69, 2005.

[10] V. N. Tsytovich, G. E. Morfill, S. V. Vladimirov, and H. M. Thomas. Elementary Physics of Complex Plasmas, volume 731 of Lecture Notes in Physics, Berlin Springer Verlag. Springer Germany, 2008.

[11] H. M. Thomas and G. E. Morfill. Melting dynamics of a plasma crystal. Nature (London), 379(6568):806-809, February 1996. 
[12] M. Bonitz. Introduction to Complex Plasmas. Springer Series on Atomic, Optical, and Plasma Physics, 2010.

[13] B. J. Berne and R. Pecora. Dynamic Light Scattering With Applications to Chemistry, Biology, and Physics. Dover Publications, Inc, 1976.

[14] R. Pecora. Dynamic Light Scattering Applications of Photon Correlation Spectroscopy. Plenum Press, 1985.

[15] C. S. Johnson and D. A. Gabriel. Laser Light Scattering. Dover Publications, 1981.

[16] H. M. Anderson. Dusty plasma studies in the gaseous electronics conference reference cell. J. Res. Natl. Inst. Stand. Technol., 100:449-462, 1995.

[17] A. J. Hurd and P. Ho. Velocity persistence of brownian particles generated in a glow discharge. Phys. Rev. Lett., 62(26):3034-, June 1989.

[18] Y. K. Khodataev, S. A. Khrapak, A. P. Nefedov, and O. F. Petrov. Dynamics of the ordered structure formation in a thermal dusty plasma. Phys. Rev. E, 57(6):7086-, June 1998.

[19] R. Borsali and R. Pecora. Soft Matter Characterisation. Springer Science+Business Media, 2008.

[20] B. Chu. Laser Light Scattering Basic Principles and Practice. Dover Publications, 1974.

[21] R. Nossal, S.-H. Chen, and C.-C. Lai. Use of laser scattering for quantitative determinations of bacterial motility. Opt. Commun., 4(1):35-39, September 1971.

[22] I. H. Hutchinson. Principles of Plasma Diagnostics. Cambridge Universtiy Press, 2002.

[23] J. B. Lastovka. Light Mixing Spectroscopy and the Spectrum of Light Scattered by Thermal Fluctuations in Liquids. PhD thesis, John Carroll University, 1967.

[24] L. Van Hove. Correlations in space and time and Born approximation scattering in systems of interacting particles. Phys. Rev., 95(1):249-, July 1954.

[25] H. M. Mott-Smith and I. Langmuir. The theory of collectors in gaseous discharges. Phys. Rev., 28(4):727-, October 1926.

[26] J. E. Allen. Probe theory - the orbital motion approach. Phys. Scr., 45(5):497-, 1992.

[27] J. Goree. Charging of particles in a plasma. Plasma Sources Sci. and Technol., 3(3):400-, 1994.

[28] A. Melzer. Introduction to Colloidal (Dusty) Plasma (Lecture Notes). Institut für Physik, Ernst-Moritz-Arndt-Universität Greifswald, 2005. 
[29] V. E. Fortov, A. V. Ivlev, S. A. Khrapak, A. G. Khrapak, and G. E. Morfill. Complex (dusty) plasmas: Current status, open issues, perspectives. Phys. Rep., 421(1-2):1-103, December 2005.

[30] G. E. Morfill and A. V. Ivlev. Complex plasmas: An interdisciplinary research field. Rev. Mod. Phys., 81(4):1353-, October 2009.

[31] E. C. Whipple. Potentials of surfaces in space. Rep. Prog. Phys., 44(11):1197-, 1981.

[32] A. Melzer, T. Trottenberg, and A. Piel. Experimental determination of the charge on dust particles forming Coulomb lattices. Phys. Lett. A, 191(3-4):301-308, August 1994.

[33] U. de Angelis. The physics of dusty plasmas. Phys. Scr., 45:465-474, 1992.

[34] S. A. Khrapak, A. V. Ivlev, and G. E. Morfill. Momentum transfer in complex plasmas. Phys. Rev. E, 70(5):056405-, November 2004.

[35] I. Bernstein. Theory of electrostatic probes in a low-density plasma. Phys. Fluids, 2(2):112-, 1959.

[36] M. Lampe. Trapped ion effect on shielding, current flow, and charging of a small object in a plasma. Phys. Plasmas, 10(5):1500-, 2003.

[37] C. K. Goertz. Dusty plasmas in the solar system. Rev. Geophys., 27:271-292, 1989.

[38] O. Havnes, C. K. Goertz, G. E. Morfill, E. Grün, and W. Ip. Dust charges, cloud potential, and instabilities in a dust cloud embedded in a plasma. J. Geophys. Res., 92(A3):2281$2287,1987$.

[39] A. Barkan, N. d'Angelo, and R. L. Merlino. Charging of dust grains in a plasma. Phys. Rev. Lett., 73(23):3093-3096, December 1994.

[40] W. Xu. Dusty plasmas: The effect of closely packed grains. J. Geophys. Res., 98:7843-7847, 1993.

[41] J. Goree, G. E. Morfill, V. N. Tsytovich, and S. V. Vladimirov. Theory of dust voids in plasmas. Phys. Rev. E, 59(6):7055-, June 1999.

[42] E. B. Tomme, D. A. Law, B. M. Annaratone, and J. E. Allen. Parabolic plasma sheath potentials and their implications for the charge on levitated dust particles. Phys. Rev. Lett., 85(12):2518-, September 2000.

[43] A. A. Uglov and A. G. Gnedovets. Effect of particle charging on momentum and heat transfer from rarefied plasma flow. Plasma Chem. and Plasma Process., 11:251-267, 1991. 
[44] I. H. Hutchinson. Ion collection by a sphere in a flowing plasma: 2. non-zero Debye length. Plasma Phys. Controlled Fusion, 45(8):1477-, 2003.

[45] I. H. Hutchinson. Ion collection by a sphere in a flowing plasma: 3. floating potential and drag force. Plasma Phys. Controlled Fusion, 47(1):71-, 2005.

[46] G. Lapenta. Simulation of charging and shielding of dust particles in drifting plasmas. Phys. Plasmas, 6(5):1442-, 1999.

[47] A. A. Sickafoose, J. E. Colwell, M. Horányi, and S. Robertson. Photoelectric charging of dust particles in vacuum. Phys. Rev. Lett., 84(26):6034-, June 2000.

[48] V. Land and W. J. Goedheer. Manipulating dust charge using ultraviolet light in a complex plasma. IEEE Trans. Plasma Sci., 35(2):280-285, 2007.

[49] V. E. Fortov, A. P. Nefedov, O. F. Petrov, A. A. Samarian, and A. V. Chernyschev. Particle ordered structures in a strongly coupled classical thermal plasma. Phys. Rev. E, 54(3):R2236-, September 1996.

[50] P. Shukla. A survey of dusty plasma physics. Phys. Plasmas, 8(5):1791-, 2001.

[51] C. Cui and J. Goree. Fluctuations of the charge on a dust grain in a plasma. IEEE Trans. Plasma Sci., 22(2):151-158, 1994.

[52] O. S. Vaulina, S. A. Khrapak, A. P. Nefedov, and O. F. Petrov. Charge-fluctuation-induced heating of dust particles in a plasma. Phys. Rev. E, 60(5):5959-, November 1999.

[53] O. Vaulina, A. Nefedov, O. Petrov, and S. Khrapak. Role of stochastic fluctuations in the charge on macroscopic particles in dusty plasmas. J. Exp. and Theor. Phys., 88:1130-1136, 1999.

[54] A. Bouchoule. Dusty Plasmas. Jhon Wiley \& Sons, 1999.

[55] R. V. Kennedy and J. E. Allen. The floating potential of spherical probes and dust grains. ii: Orbital motion theory. J. Plasma Phys., 69(06):485-506, 2003.

[56] S. Khrapak and G. Morfill. Basic processes in complex (dusty) plasmas: Charging, interactions, and ion drag force. Contr. Plasma Phys., 49(3):148-168, 2009.

[57] M. Lampe, V. Gavrishchaka, G. Ganguli, and G. Joyce. Effect of trapped ions on shielding of a charged spherical object in a plasma. Phys. Rev. Lett., 86(23):5278-5281, June 2001.

[58] T. Trottenberg, A. Melzer, and A. Piel. Measurement of the electric charge on particulates forming Coulomb crystals in the sheath of a radiofrequency plasma. Plasma Sources Sci. and Technol., 4(3):450, 1995. 
[59] M. Lampe. Interactions between dust grains in a dusty plasma. Phys. Plasmas, 7(10):3851, 2000.

[60] S. A. Maiorov, S. V. Vladimirov, and N. F. Cramer. Plasma kinetics around a dust grain in an ion flow. Phys. Rev. E, 63(1):017401-, December 2000.

[61] D. Lemons. Two-dimensional wake potentials in sub- and supersonic dusty plasmas. Phys. Plasmas, 7(6):2306-, 2000.

[62] G. Lapenta. Linear theory of plasma wakes. Phys. Rev. E, 62(1):1175-, July 2000.

[63] D. Winske. Nonlinear wake potential in a dusty plasma. IEEE Trans. Plasma Sci., 29(2):191-197, 2001.

[64] G. Lapenta. Nature of the force field in plasma wakes. Phys. Rev. E, 66(2):026409-, August 2002.

[65] S.V. Vladimirov. Molecular dynamics simulation of plasma flow around two stationary dust grains. Phys. Plasmas, 10(10):3867-, 2003.

[66] A. Melzer, V. A. Schweigert, and A. Piel. Transition from attractive to repulsive forces between dust molecules in a plasma sheath. Phys. Rev. Lett., 83(16):3194-, October 1999.

[67] M. S. Barnes, J. H. Keller, J. C. Forster, J. A. O'Neill, and D. K. Coultas. Transport of dust particles in glow-discharge plasmas. Phys. Rev. Lett., 68(3):313-316, January 1992.

[68] S. A. Khrapak, A. V. Ivlev, G. E. Morfill, and H. M. Thomas. Ion drag force in complex plasmas. Phys. Rev. E, 66(4):046414-, October 2002.

[69] S. A. Khrapak, A. V. Ivlev, G. E. Morfill, and S. K. Zhdanov. Scattering in the attractive Yukawa potential in the limit of strong interaction. Phys. Rev. Lett., 90(22):225002-, June 2003.

[70] S. Hamaguchi and R. T. Farouki. Polarization force on a charged particulate in a nonuniform plasma. Phys. Rev. E, 49(5):4430-, May 1994.

[71] P. S. Epstein. On the resistance experienced by spheres in their motion through gases. Phys. Rev., 23(6):710-, June 1924.

[72] B. Liu, J. Goree, V. Nosenko, and L. Boufendi. Radiation pressure and gas drag forces on a Melamine-Formaldehyde microsphere in a dusty plasma. Phys. Plasmas, 10(1):9-20, January 2003.

[73] O. Arp, D. Block, A. Piel, and A. Melzer. Dust Coulomb balls: Three-dimensional plasma crystals. Phys. Rev. Lett., 93(16):165004-, October 2004. 
[74] S. Hamaguchi, R. T. Farouki, and D. H. E. Dubin. Triple point of Yukawa systems. Phys. Rev. E, 56(4):4671-, October 1997.

[75] M. Stevens. Melting of Yukawa systems: A test of phenomenological melting criteria. Chem. Phys., 98(3):2319-, 1993.

[76] E. J. Meijer. Melting line of Yukawa systems by computer simulation. Chem. Phys., 94:2269-, 1991.

[77] O. Vaulina, S. Khrapak, and G. Morfill. Universal scaling in complex (dusty) plasmas. Phys. Rev. E, 66(1):016404-, July 2002.

[78] N. N. Rao, P. K. Shukla, and M. Y. Yu. Dust-acoustic waves in dusty plasmas. Planet. Space Sci., 38(4):543-546, April 1990.

[79] A. V. Ivlev, S. K. Zhdanov, B. A. Klumov, V. N. Tsytovich, U. de Angelis, and G. E. Morfill. Kinetics of ensembles with variable charges. Phys. Rev. E, 70(6):066401-, December 2004.

[80] A. V. Ivlev. Generalized kinetic theory of ensembles with variable charges. Phys. Plasmas, 12(9):092104-, 2005.

[81] S. K. Zhdanov. Non-Hamiltonian dynamics of grains with spatially varying charges. Phys. Plasmas, 12(7):072312-, 2005.

[82] S. Nunomura, T. Misawa, N. Ohno, and S. Takamura. Instability of dust particles in a Coulomb crystal due to delayed charging. Phys. Rev. Lett., 83(10):1970-, September 1999.

[83] F. Melandsø. Heating and phase transitions of dust-plasma crystals in a flowing plasma. Phys. Rev. E, 55(6):7495-, June 1997.

[84] A. Aschinger. Struktur und Dynamik von Plasma Kristallen, Diploma thesis, 2008.

[85] K. Statsenko, A. Ivanov, I. Shakhova, A. Gavrikov, Y. Khrustalyev, O. Petrov, and V. Fortov. The measurement of kinetic temperature of dusty component of complex plasma in RF-discharge. AIP Conf. Proc., 799(1):438-441, October 2005.

[86] K. Schätzel. Correlation techniques in dynamic light scattering. Appl. Phys. B: Laser Opt., 42:193-213, 1987.

[87] S. Chen and B. Chu. Scattering Technique Applied to Supramolecular and Nonequilibrium Systems. Plenum Press - New York and London, 1981.

[88] E. Jakeman. Measurements of the factorization properties of higher-order optical correlation functions. J. Phys. A: Gen. Phys., Vol.1:497-499, 1968. 
[89] M. R. Zachariah, D. Chin, H. G. Semerjian, and J. L. Katz. Dynamic light scattering and angular dissymmetry for the in situ measurement of silicon dioxide particle synthesis in flames. Appl. Opt., 28(3):530-536, February 1989.

[90] O. Vaulina, A. Samarian, O. Petrov, B. James, and F. Melandsø. Formation of vortex dust structures in inhomogeneous gas-discharge plasmas. Plasma Phys. Rep., 30(11):918-936, 2004.

[91] O. S. Vaulina and S. V. Vladimirov. Diffusion and dynamics of macro-particles in a complex plasma. Phys. Plasmas, 9(3):835-840, 2002.

[92] O. F. Petrov, A. P. Nefedov, and V. E. Fortov. Thermal dusty plasmas: diagnostics and results of investigations. Phys. Scr., 2001(T89):25, 2001. 


\section{Danksagung}

Ich möchte Herrn Prof. Dr. J. Winter für die Möglichkeit diese Arbeit durchführen zu können meinen besonderen Dank aussprechen. Mir wurden immer genügend Freiheiten gelassen, um eigenen Ansätzen und Gedanken nachzugehen. Der Austausch und die Reflexion neuer Ideen in diversen Treffen haben mir immer wieder Schwung gegeben und die weitere Arbeit inspiriert.

Herrn Dr. M. Böke danke ich für den kompetenten Beitrag zur Lösung experimenteller Probleme und dem fundierten Dazutun in physikalischen Dingen. Des Weiteren danke ich für die Hilfe in organisatorischen und verwaltungstechnischen Fragen.

Herrn Dr. Volker Schulz-von der Gathen danke für Rat und Tat in experimentellen Dingen und für den Zuspruch und die Unterstützung darüber hinaus.

Brankica Sikimić danke ich für die konstruktiven Diskussionen zum Thema staubige Plasmen und den Austausch über experimentelle Dinge. Die Anteilnahme und Ratschläge haben mich oft motiviert und mich weiter voran gebracht.

Frau Verena M. Scharf und Dr. Henrik Böttner danke ich recht herzlich für die schnelle und akkurate Revision des Manuskripts.

Dr. Jens Ränsch danke ich für die Einarbeitung in das Fachgebiet der komplexen Plasmen und für die exzellente Betreuung während der Diplomarbeit und zu Beginn dieser Arbeit.

Meinen Kollegen am Lehrstuhl für Experimentalphysik II einschließlich der Arbeitsgruppe Reaktive Plasmen danke ich für ein tolles Arbeitsklima und für die gemütlichen Zusammenkünfte bei Kaffee und Kuchen.

Frau M. Ocklenburg danke ich für den Beistand in Sachen Organisation und Verwaltung.

Ich möchte den beteiligten Technikern des Instituts, Herrn A. Lang, Herrn K. Fiegler, Herrn B. Redeker, Herrn M. Konkowski, Herrn K. Brinkhoff und Herrn W. Winterhalder für die vielen kleinen und großen Beiträge zu dem experimentellen Aufbau danken. Des Weiteren geht mein Dank an die Mitarbeiter der Mechanikwerkstatt. Ohne die hervorragende technische Unterstützung wäre dies Arbeit nicht möglich gewesen.

Ein großer Dank geht an meine Eltern die mir das Studium der Physik überhaupt erst ermöglichten und mir immer einen festen Rückhalt in Krisenzeiten boten. 


\section{Lebenslauf}

\section{Persönliche Angaben}

$\begin{array}{ll}\text { Name } & \text { Andreas Aschinger } \\ \text { Geburtsdatum } & 08.09 .1981 \\ \text { Geburtsort } & \text { Schleswig }\end{array}$

\section{Hochschulausbildung}

seit 07.2008

Juni 2008

$04.2007-06.2008$

$04.2006-04.2007$

$10.2002-04.2006$

$10.2001-10.2002$
Promotionsvorhaben über komplexe Plasmen und Beschäftigung als wissenschaftlicher Mitarbeiter

Lehrstuhl für Experimentalphysik II an der Ruhr-Universität Bochum

Abschluss: Diplom Physik

Titel der Diplomarbeit: Struktur und Dynamik von Plasmakristallen

Studium der Physik: Studium komplexer Plasmen und Anfertigung der Diplomarbeit

Beschäftigung als studentische Hilfskraft

Lehrstuhl für Experimentalphysik II an der Ruhr-Universität Bochum

Studium der Physik: Spezialisierung auf den Forschungsbereich Plasmaphysik

Lehrstuhl für Experimentelle Plasmaphysik an der Humboldt-Universität Berlin

Aufnahme des Diplomstudienganges Physik

Universität Hamburg

Studium der Bioinformatik

Universität zu Lübeck

\section{Schulausbildung}

$04.1998-07.2001$

$04.1992-03.1998$

$04.1988-03.1992$
Fachgymnasium mit Schwerpunkt Wirtschaft

Handelslehranstalt Flensburg

Heinrich-Andresen Gemeinschaftsschule Sterup

Heinrich-Andresen Grundschule Sterup 
Aschinger, Andreas

Name, Vorname

Versicherung gemäß § 7 Abs. 2 Nr. 5 PromO 1987

Hiermit versichere ich, dass ich meine Dissertation selbstständig angefertigt und verfasst und keine anderen als die angegebenen Hilfsmittel und Hilfen benutzt habe. Meine Dissertation habe ich in dieser oder ähnlicher Form noch bei keiner anderen Fakultät der Ruhr-Universität Bochum oder bei einer anderen Hochschule eingereicht.

Bochum, den

A. Aschinger 